DOE/EIA-0607(96)

Distribution CategonyC-950

\title{
Issues in Midterm Analysis and Forecasting 1996
}

\section{August 1996}

Energy Information Administration

Office of Integrated Analysis and Forecasting

U.S. Department of Energy

Washington, DC 20585 


\section{Preface}

Issues in Midterm Analysis and Forecasting 1996 (Issues) presents a series of five papers, which cover topics in analysis and modeling that underlie the Annual Energy Outlook 1996 (AEO96). The AEO96 (DOE/EIA-0383(96)), published in January 1996, presents national forecasts of energy production, demand, imports, and prices through the year 2015. The AEO96 includes forecasts of energy markets for five cases-a reference case and four additional cases that assume higher and lower economic growth and higher and lower world oil prices than in the reference case. The forecasts are generated using the Energy Information Administration's (EIA) National Energy Modeling System (NEMS).

The papers included in Issues are intended to describe underlying analyses for the projections in the AEO96 and other analytical products of the EIA Office of Integrated Analysis and Forecasting. This provides public access to analytical work done in preparation for the AEO96 and to other unpublished analyses. Specific topics were chosen for their relevance to current energy issues or to highlight modeling activities in NEMS.

The AEO96 projections are used by Federal, State, and local governments, trade associations, and other planners and decisionmakers in the public and private sectors. They are published in accordance with Section 205(c) of the Department of Energy Organization Act of 1977 (Public Law 95-91), which requires the Administrator of EIA to prepare an annual report that contains trends and projections of energy consumption and supply. The projections in $A E O 96$ are not statements of what will happen, but what might happen given the specific assumptions and methodologies used. These projections provide an objective, policy-neutral reference case that can be used to analyze policy initiatives. As a policyneutral data and analysis organization, EIA does not propose, advocate, or speculate on future legislative and regulatory changes. The projections assume that laws remain as currently enacted, so that policy initiatives can be analyzed relative to the $A E O$ forecasts. Assuming current legislation and regulations, even knowing that changes will occur, will naturally result in projections that differ from the final data.

Issues was prepared under the direction of Mary J. Hutzler (mhutzler@eia.doe.gov, 202/586-2222), Director of the Office of Integrated Analysis and Forecasting;
Arthur T. Andersen (aanderse@eia.doe.gov, 202/ 5861441), Director of the Energy Demand and Integration Division; Scott B. Sitzer (ssitzer@eia.doe.gov, 202/5862308), Director of the Energy Supply and Conversion Division; and Andy S. Kydes (akydes@eia.doe.gov, 202/586-2222), Senior Modeling Analyst. The publication was coordinated by Susan H. Shaw (sshaw@eia.doe.gov, 202/586-4838). Specific questions about the papers in Issues may be addressed to the following authors:

The Potential Impact of Technological Progress on U.S. Energy Markets: Andy S. Kydes (akydes@eia.doe.gov, 202/586-2222)

The Outlook for U.S. Import Dependence: Bruce H. Bawks (bbawks@eia.doe.gov, 202/586-6579)

Fuel Economy, Vehicle Choice, and Changing Demographics: Barry N. Cohen (bcohen@eia.doe.gov, 202/586-5359)

Annual Energy Outlook Forecast Evaluation: Scott B. Sitzer (ssitzer@eia.doe.gov, 202/586-2308)

Differences Between Energy Information Administration Energy Forecasts: Reasons and Resolution: Arthur T. Andersen (aanderse@eia.doe.gov, 202/586-1441)

Issues will be available on the November release of the EIA CD-ROM and on the EIA Home Page on the Internet (http://www.eia.doe.gov) by September 1996. The AEO96, assumptions underlying the projections in the $A E O 96$, and tables of regional and other detailed results from the AEO96 forecasts are available on the CD-ROM and on the EIA Home Page. Forecast tables for the five cases in the AEO96 are also available via modem on EIA's Electronic Publication (EPUB) System (202/586-2557) and on diskette. To obtain the AEO96 diskettes, contact the Office of Scientific and Technical Information by telephone at $615 / 576-8401$ or by mail at P.O. Box 62, Oak Ridge, TN 37831. The National Energy Modeling System: An Overview (DOE/EIA-0581(96)), which provides a summary description of NEMS, is available on the CD-ROM and on the EIA Home Page. Model documentation reports for NEMS are available on the CD-ROM. 


\section{DISCLAIMER}

This report was prepared as an account of work sponsored by an agency of the United States Government. Neither the United States Government nor any agency thereof, nor any of their employees, makes any warranty, express or implied, or assumes any legal liability or responsibility for the accuracy, completeness, or usefulness of any information, apparatus, product, or process disclosed, or represents that its use would not infringe privately owned rights. Reference herein to any specific commercial product, process, or service by trade name, trademark, manufacturer, or otherwise does not necessarily constitute or imply its endorsement, recommendation, or favoring by the United States Government or any agency thereof. The views and opinions of authors expressed herein do not necessarily state or reflect those of the United States Government or any agency thereof. 


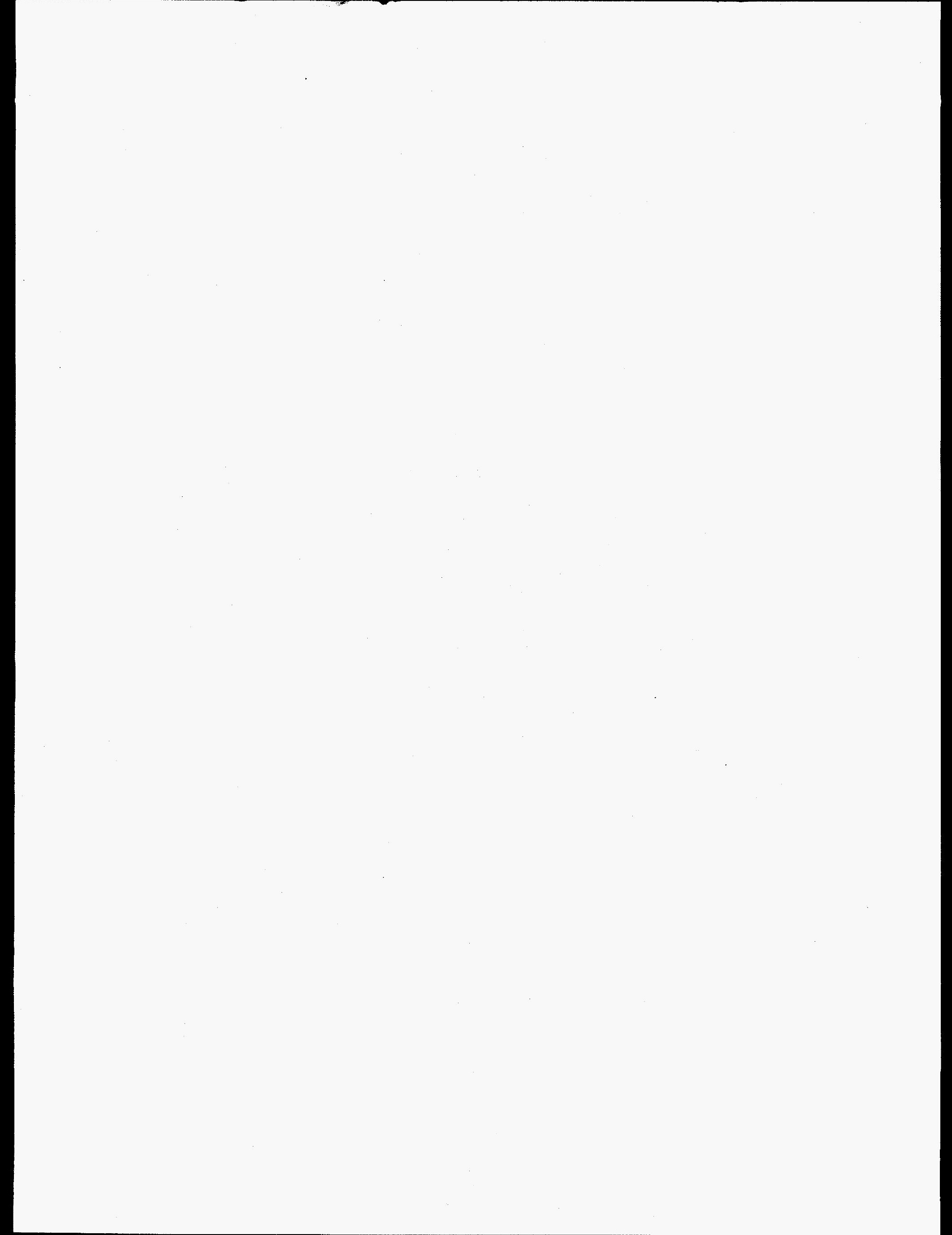




\section{DISCLAIMER}

Portions of this document may be illegible in electronic image products. Images are produced from the best available original document. 


\section{Contents}

The Potential Impact of Technological Progress on U.S. Energy Markets $\ldots \ldots \ldots \ldots \ldots \ldots \ldots \ldots$

\section{Tables}

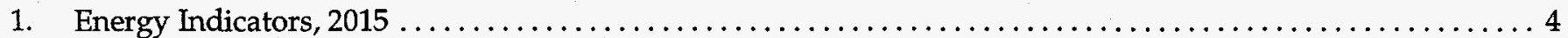

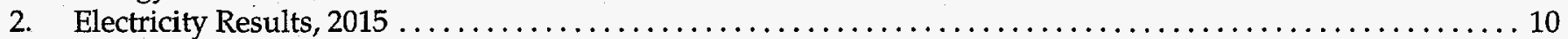

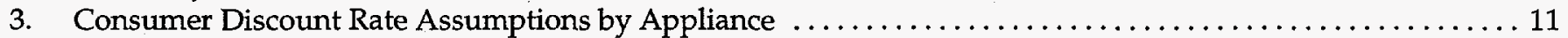

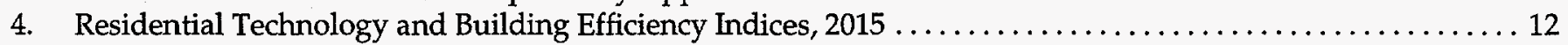

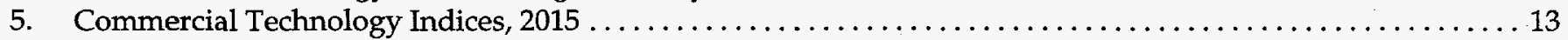

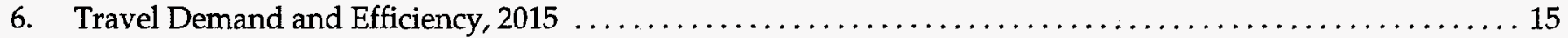

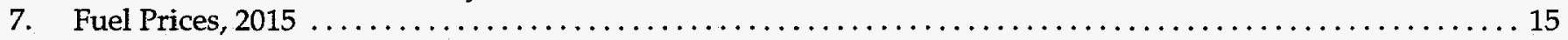

\section{Figures}

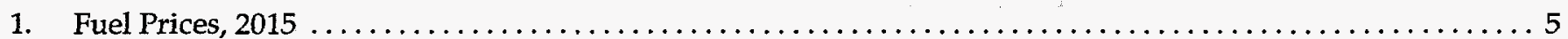

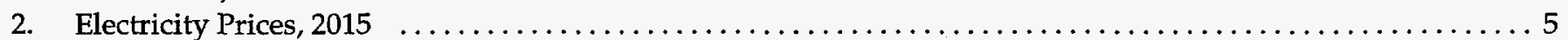

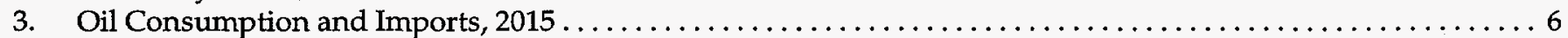

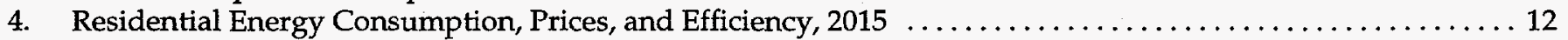

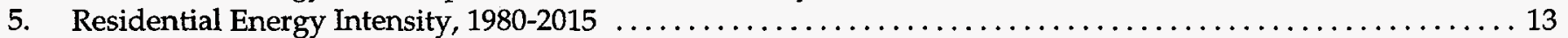

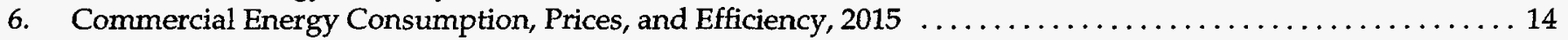

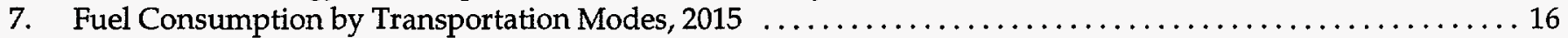

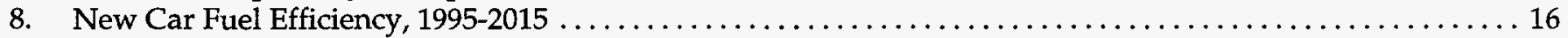

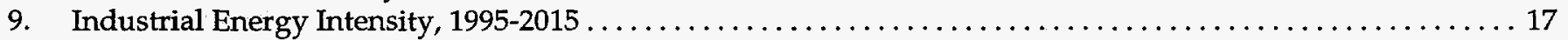

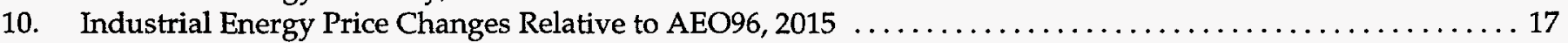

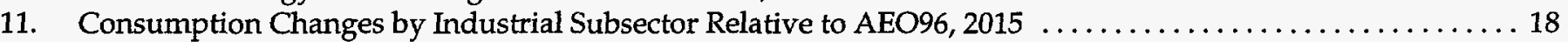

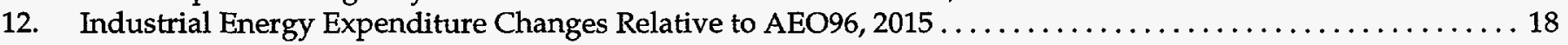

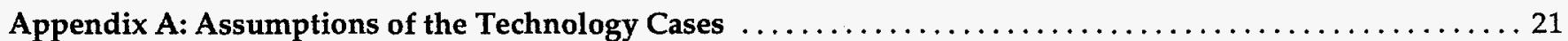

Appendix B: Comparison Tables Illustrating the Slow Technological Progress Case, the AEO96

Reference Case, and the Rapid Technological Progress Case $\ldots \ldots \ldots \ldots \ldots \ldots \ldots \ldots \ldots \ldots \ldots \ldots \ldots \ldots \ldots$

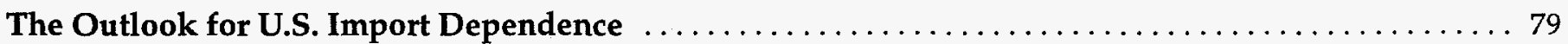

\section{Figures}

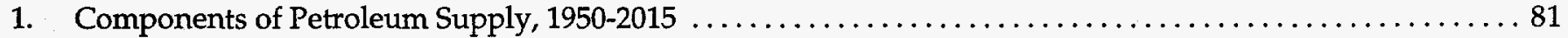

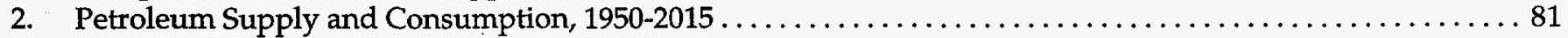

3. Crude Oil and Product Net Imports as a Percentage of Consumption, $1950-2015 \ldots \ldots \ldots \ldots \ldots \ldots \ldots 2$

4. Net Import Percentage of Consumption, Oil Price Cases, All Countries and Persian Gulf, 1995-2015 . . . . . . 82

5. Net Import Percentage of Consumption, Reference Case, All Countries, Persian Gulf, and with Canada

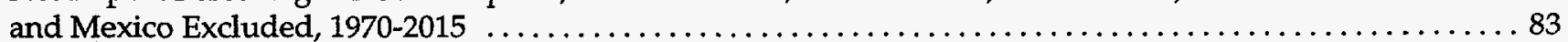




\section{Contents (Continued)}

Fuel Economy, Vehicle Choice, and Changing Demographics $\ldots \ldots \ldots \ldots \ldots \ldots \ldots \ldots \ldots \ldots$

Tables

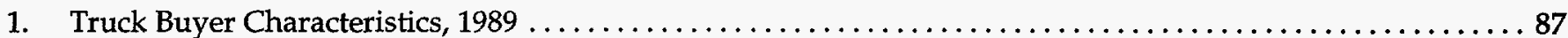

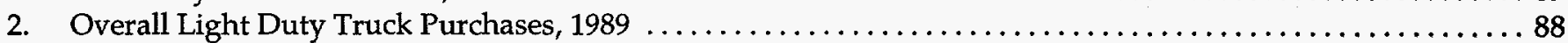

\section{Figures}

1. Age and Sex Weighted Population Index of Truck Purchasers, $1970-1995 \ldots \ldots \ldots \ldots \ldots \ldots \ldots \ldots \ldots \ldots$

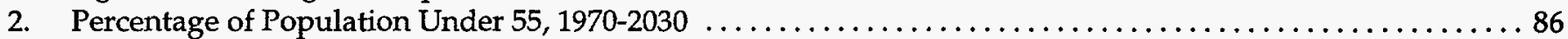

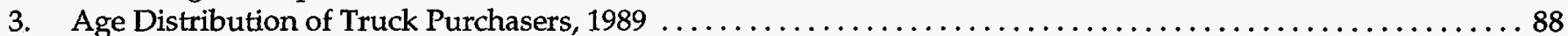

4. Cumulative Percentage of Truck Purchases by Age Category, $1989 \ldots \ldots \ldots \ldots \ldots \ldots \ldots \ldots \ldots \ldots \ldots \ldots \ldots$

5. Age and Sex Weighted Population Index of Truck Purchasers, $1970-2030 \ldots \ldots \ldots \ldots \ldots \ldots \ldots \ldots$. . . . . . . 89

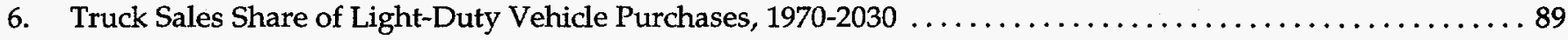

Annual Energy Outlook Forecast Evaluation $\ldots \ldots \ldots \ldots \ldots \ldots \ldots \ldots \ldots \ldots \ldots \ldots \ldots \ldots \ldots \ldots \ldots$

Tables

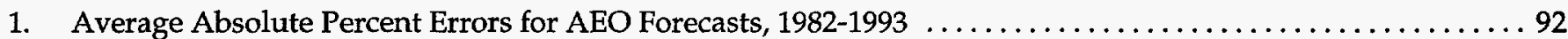

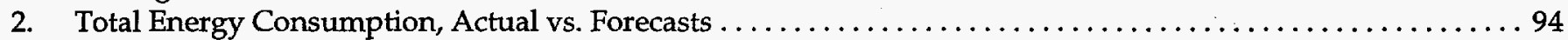

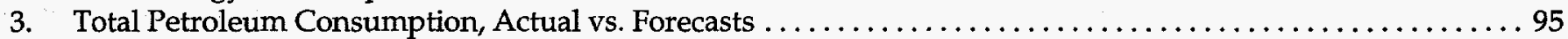

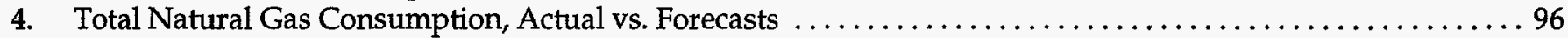

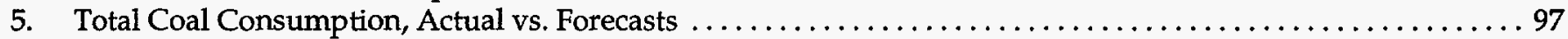

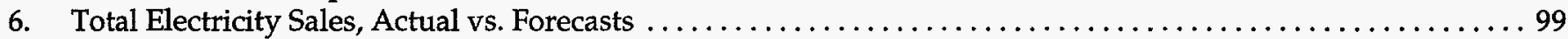

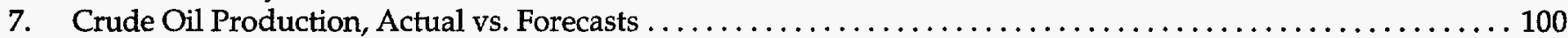

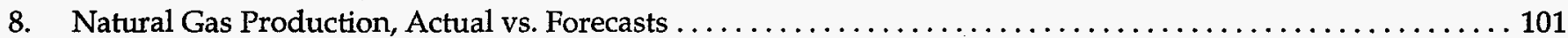

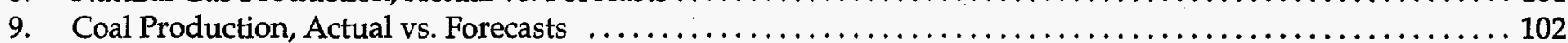

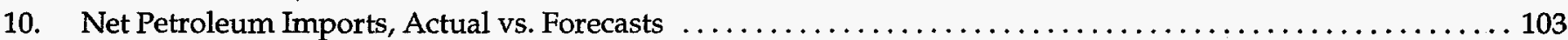

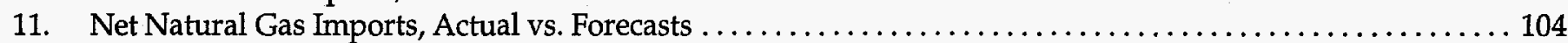

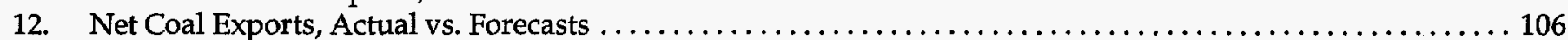

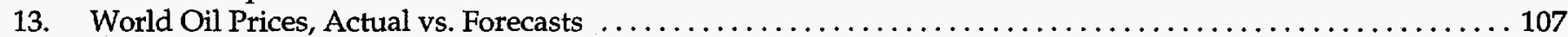

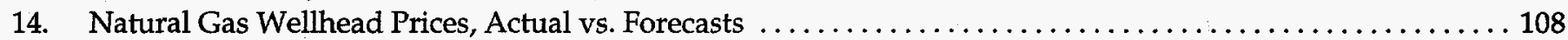

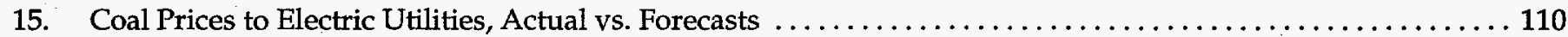

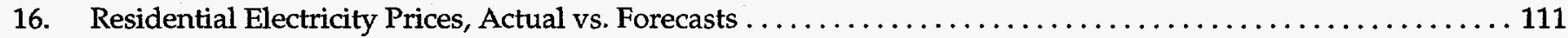

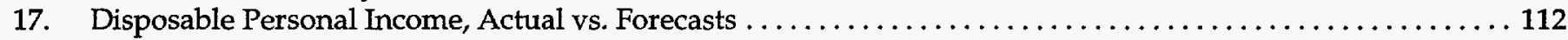

\section{Differences Between Energy Information Administration Energy Forecasts: Reasons and Resolution 115}

\section{Tables}

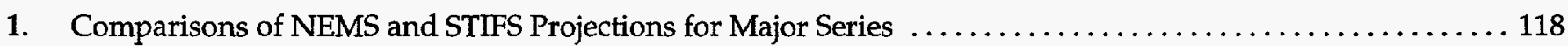

2. NEMS and STIFS Projections in 1994 and Percent Differences ............................... 119

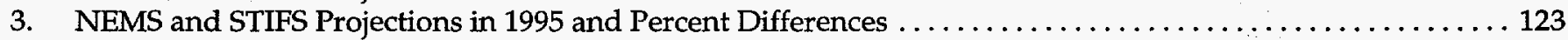




\title{
The Potential Impact of Technological Progress on U.S. Energy Markets
}

\author{
by \\ Erin Boedecker, John Cymbalsky, Crawford Honeycutt, Jeffrey Jones, \\ Andy S. Kydes and Duc Le
}

The following report represents a pilot study on the potential impact of technological progress on the U.S. economy and environment. For simplicity, it used the special technology cases developed in the Annual Energy Outlook 1996 and combined them to form integrated technology cases which are likely to produce more realistic scenarios than standalone cases.

Since "technological optimism" is treated differently in each energy sector, the level of optimism across sectors is not consistent and therefore does not produce a level playing field across energy markets. This analysis does not attempt to evaluate the energy programs of the Federal Government or their programmatic goals. There is no relationship between the assumptions in the cases analyzed below and the funding levels of the U.S. research and development programs.

We invite technical reviews of this effort so that we might improve both the definitions of the technology cases and their implementation and make meaningful comparisons across markets and technologies in order to analyze their future impacts on energy markets and the environment.

\section{Introduction}

Technological progress, which we define in this paper to include technological innovation and/or adoption, and the rates of supply productivity improvements have been major factors that have often been underestimated since the mid-1970s by energy forecasters. As a consequence of this underestimation, supply projections have been dominated by depletion effects and the resulting fossil fuel price projections have had high rates of growth-neither of which have been borne out by history. The rate of technological progress has historically far outpaced the potential depletion effects on U.S. domestic production, and that technological progress has been the principal reason for coal and natural gas price stability or declines over the past fifteen years. Technological progress on the supply side could continue to mitigate depletion effects through 2015 and beyond.

More recently, the apparent need for carbon mitigation has further focused attention on the potential role that advanced new technologies may play in achieving compliance with international carbon stabilization goals. International meetings and conferences on climate change consistently focus on the potential role of advanced technologies as the centerpiece of any carbon mitigation and climate stabilization strategy.

These factors, combined with workshops held at the Energy Information Administration (EIA), indicate that integrated technology cases are of value to a broad base of industry, Federal, and Departmental customers. While the technology study focus is important, the formulation and assessment of integrated technology cases is a relatively new and untested analytical activity at EIA.

This study does not attempt to evaluate the energy programs of the Federal government or their programmatic goals. If programmatic goals of Federal research and development (R\&D) are achieved, increased energy savings and supply above those indicated in this report could be realized. This study does not attempt to model those programmatic goals. Further, future geopolitical events, e.g., greater instability in international energy markets, or environmental imperatives, e.g., binding commitments to reduce carbon emissions, could cause an increased focus on end-use and supply improvement and implementation than represented by this study. 
Because technological optimism is treated differently in each module of the National Energy Modeling System (NEMS), no attempt was made in this study to level the playing field in terms of insuring the same level of technological optimism across markets. Thus, this analysis should not be used to imply that additional $R \& D$ would be more effective in one area versus another. In addition, the analysis process was complicated because some modules, like the oil and gas supply module, represent technological progress autonomously with no explicit representation of technologies while others, like the residential, commercial, and electricity generation modules, represent technologies explicitly. Even within technology-rich modules, technological progress is represented in some cases as a greater willingness by consumers to adopt energy-efficient technologies from the technologies represented in the Annual Energy Outlook 1996 (AEO96) $^{1}$ reference case, for example, in the residential and commercial sectors, while in other modules, the characterization of technologies was actually changed to reflect greater or lesser optimism, such as in the generation module. In the residential and commercial sectors, we judged that a dramatically increased willingness to adopt advanced technologies from the menu of AEO96 reference case technologies would have a much greater impact on energy use patterns in these sectors than keeping consumer purchase patterns unchanged and making available higher efficiency end-use equipment options. Consequently, comparisons or conclusions across technology modules should not be drawn casually because of such concerns. This pilot study was initiated to better understand the impact of technology on energy and the environment and to obtain customer feedback in order to improve on the construction of the cases for further analysis.

The AEO96 defined a series of sensitivity cases for select energy sectors, also referred to as standalone technology cases, ${ }^{2}$ that examined the impact of alternative assumptions for that sector, all else held constant to the AEO96 reference case. Two sets of market and technology assumptions were defined for each energy sector to illustrate uncertainty in these markets. In this study, the series of AEO96 slow technology assumptions were combined to form an integrated slow technological progress case. The more optimistic group of sensitivity assumptions was also combined to form an integrated rapid technological progress case. The slow technological progress case examined fuel and technology choices when the availability or adoption of new technologies was assumed to occur at a slower rate than the AEO96 reference case in all energy markets. The rapid technological progress case examined fuel and technology choices when the availability or adoption of new technologies was assumed to occur at a faster rate than the AEO96 reference case. Although the rapid technology case incorporates either faster adoption or faster advancement of cost and performance of technologies, the study did not attempt to analyze the potentially best technologies achievable through research, and it does not incorporate all possible new technologies or the greatest possible improvements in new technologies. The assumptions behind the cases are described in Appendix A.

The reader should be aware of one additional major assumption of this study. Technology changes and market effects were assumed to be isolated to U.S. markets only. Including international markets would probably have resulted in even more significant oil price impacts.

This paper examines the implications of alternative technological assumptions of the AEO96 sensitivity cases within an integrated energy-economic framework. The benefits-usually energy savings or price reductions-of many energy programs are usually estimated by program offices in a nonintegrated framework, analyzing technologies only in their direct market without considering feedback effects. Integrated assessment of technology programs occasionally occurs in offices within the same umbrella departmental organization, but outside the program offices. When the analytical framework is not integrated, the potential interactions and rebound effects between programs and technologies may not be adequately accounted for and different impacts are likely to be estimated for an individual program than when these other factors are considered.

The goals of this analysis are: (a) to analyze the potential role of technological progress on energy supply, consumption, and prices in U.S. energy markets; and (b) to assess how "success" on one side of the supply or demand equation may reduce the potential benefits on the other side.

"Success" for end-use demand means that consumers are more willing to purchase high-efficiency equipment that requires longer payback periods than in the AEO96 reference case. Success in the electricity generation markets is reflected by earlier availability of advanced technologies, lower perceived risk and risk premium, and

\footnotetext{
${ }^{1}$ Energy Information Administration, Annual Energy Outlook 1996, DOE/EIA-0383(96) (Washington, DC, January 1996).

${ }^{2}$ Energy Information Administration, Annual Energy Outlook 1996, DOE/EIA-0383(96) (Washington, DC, January 1996), pp. 250-259.
} 
faster "learning-by-doing" which can lower costs to the mature state sooner. For oil and gas production, success is represented as faster rates of drilling cost reduction and expansion of the resource base. For coal, success is represented as higher labor productivity rates. Slower technological progress is typically represented for demand by freezing the menu of technologies to that available in 1995. For electricity generation technologies, advanced technologies become available later in the forecast, at higher cost, and slower learning (more cumulative capacity is required to be constructed to achieve the same cost reductions achieved in the AEO96 reference case). It should be noted that technologies and their characteristics represented in the AEO96 reference case are considered to be conservative by some analysts.

The National Energy Modeling System (NEMS) was used to perform the simulations. The integrated technology cases examined were defined as follows:

(a) rapid technological progress assumptions for both end-use and supply technologies

(b) slow technological progress assumptions for both end-use and supply technologies

(c) rapid technological progress assumptions only for end-use technologies with reference case assumptions for supply technologies (electricity generation technologies are incorporated in the supply technology progress assumptions)

(d) rapid technological progress assumptions only for supply technologies with reference case demand technology assumptions

(e) slow technological progress assumptions for enduse technologies with reference case assumptions for supply technologies

(f) slow technological progress assumptions for supply technologies with reference case demand technology assumptions

(g) rapid technological progress assumptions for supply technologies with slow technological progress assumptions for the demand technologies

(h) slow technological progress assumptions for supply technologies with rapid technological progress assumptions for the demand technologies.

All cases used the AEO96 reference case world oil price assumptions, U.S. macroeconomic growth assumptions, and international market conditions. The results of cases (a) and (b) are compared to the $A E O 96$ reference case in Appendix B.

Although alternative supply technologies are incorporated in the cases and some discussion of the price impacts of the alternative supply technologies are discussed, this study focuses on demand and electricity markets.

\section{Energy Supply, Consumption, and Price Patterns}

The AEO96 reference case indicates where technological progress may proceed, given today's policies, consumer behavior trends, and other economic and noneconomic conditions. The rate of technological progress toward higher-efficiency and lower-cost energy supply and utilization technologies and their rate of market adoption can have a profound effect on energy prices, energy consumption, and carbon emissions. Although technological progress for both the supply and demand technologies in the energy system can result in significant price reductions, the impacts of simultaneous technological progress for both supply and demand on prices are not additive. Technological progress for supply technologies primarily reduces the cost of production and, consequently, improves the competitiveness of domestic energy industries and provides greater self-reliance on domestically-produced energy supplies. Since energy is less than 10 percent of the Gross Domestic Product (GDP), reductions in energy prices provide only a mild positive influence on economic growth and lower inflation rates. Table 1 summarizes the results of the integrated technology cases.

Technological progress for demand technologies primarily reduces energy consumption and, as a consequence, energy prices and carbon emissions. In general, however, individual technological successes and benefits are not necessarily additive because of the energy-economic feedback effects and the interactions among energy decisionmakers across energy markets. To illustrate, the wellhead natural gas price in 2015 is projected to be $\$ 1.86$ per thousand cubic feet in 1994 dollars when rapid demand technological progress assumptions are combined with $A E O 96$ reference case supply technology assumptions (Figure 1). Conversely, when rapid supply technological progress assumptions are combined with AEO96 reference case demand technological progress assumptions, the wellhead gas price is projected to be $\$ 1.98$ per thousand cubic feet. The wellhead gas price is projected to be $\$ 1.55$ per thousand cubic feet when rapid technological progress is combined for both supply and demand. If the technological impacts were additive, the 
Table 1. Energy Indicators, 2015

\begin{tabular}{|c|c|c|c|c|c|c|c|c|c|c|}
\hline Key Indicators & 1994 & $\begin{array}{c}\text { AEO96 } \\
\text { Reference } \\
\text { Case } \\
\end{array}$ & $\begin{array}{c}\text { Rapid } \\
\text { Technological } \\
\text { Progress } \\
\end{array}$ & $\begin{array}{c}\text { Slow } \\
\text { Technological } \\
\text { Progress } \\
\end{array}$ & $\begin{array}{c}\text { Rapid } \\
\text { Supply and } \\
\text { Reference } \\
\text { Demand } \\
\end{array}$ & $\begin{array}{c}\text { Reference } \\
\text { Supply and } \\
\text { Rapid } \\
\text { Demand } \\
\end{array}$ & $\begin{array}{c}\text { Slow Supply } \\
\text { and } \\
\text { Reference } \\
\text { Demand } \\
\end{array}$ & $\begin{array}{c}\text { Reference } \\
\text { Supply } \\
\text { and Slow } \\
\text { Demand } \\
\end{array}$ & $\begin{array}{l}\text { Rapid } \\
\text { Supply } \\
\text { and Slow } \\
\text { Demand }\end{array}$ & $\begin{array}{c}\text { Slow } \\
\text { Supply } \\
\text { and Rapid } \\
\text { Demand }\end{array}$ \\
\hline $\begin{array}{l}\text { World Oil Price } \\
\text { (1994 dollars per barrel) }\end{array}$ & 15.52 & 25.43 & 21.17 & 28.58 & 24.06 & 22.25 & 26.77 & 26.63 & 25.41 & 23.24 \\
\hline $\begin{array}{l}\text { Oil Imports } \\
\text { (quadrillion Btu) }\end{array}$ & 17.3 & 25.4 & 19.4 & 29.3 & 23.5 & 21.1 & 27.1 & 27.0 & 25.1 & 22.7 \\
\hline $\begin{array}{l}\text { Oil Consumption } \\
\text { (quadrillion Btu) }\end{array}$ & 34.6 & 41.7 & 35.9 & 44.7 & 41.6 & 35.9 & 42.0 & 44.0 & 43.6 & 36.1 \\
\hline $\begin{array}{l}\text { Gas Wellhead Price } \\
\text { (1994 dollars per thousand } \\
\text { cubic feet) } \quad \ldots \ldots \ldots \ldots .\end{array}$ & 1.88 & 2.57 & 1.55 & 4.18 & 1.98 & 1.86 & 3.63 & 3.12 & 2.17 & 2.25 \\
\hline $\begin{array}{l}\text { Gas Consumption } \\
\text { (quadrillion Btu) }\end{array}$ & 21.4 & 29.5 & 25.4 & 29.4 & 30.0 & 25.1 & 28.4 & 31.1 & 31.3 & 24.4 \\
\hline $\begin{array}{l}\text { Coal Minemouth Price } \\
\text { (1994 dollars per ton) }\end{array}$ & 19.41 & 17.39 & 15.26 & 20.95 & 16.22 & 16.09 & 19.07 & 18.68 & 17.74 & .17 .44 \\
\hline $\begin{array}{l}\text { Coal Consumption } \\
\text { (quadrillion Btu) }\end{array}$ & 19.7 & 23.3 & 21.2 & 26.0 & 22.7 & 21.4 & 23.7 & 25.1 & 24.7 & 21.6 \\
\hline $\begin{array}{l}\text { Total Consumption } \\
\text { (quadrillion Btu) }\end{array}$ & 89.1 & 108.0 & 95.5 & 113.7 & 108.4 & 95.2 & 107.5 & 114.1 & 114.1 & 94.9 \\
\hline $\begin{array}{l}\text { Carbon Emissions } \\
\text { (millions tons) }\end{array}$ & 1,397 & 1,735 & 1,540 & 1,863 & 1,728 & 1,540 & 1,737 & 1,847 & 1,837 & 1,540 \\
\hline $\begin{array}{l}\text { Per Capita Carbon Emissions } \\
\text { (tons) } \quad \ldots \ldots \ldots \ldots \ldots \ldots . .\end{array}$ & 5 & 5.6 & 5.0 & 6.0 & 5.6 & 5.0 & 5.6 & 5.9 & 5.9 & 5.0 \\
\hline GDP (billion 1987 dollars) & 5,344 & 8,114 & 8,158 & 8,072 & 8,130 & 8,148 & 8,090 & 8,102 & 8,118 & 8,135 \\
\hline
\end{tabular}

AEO = Annual Energy Outlook.

$B t u=$ British thermal unit.

GDP $=$ Gross Domestic Product.

Source: Tables B1, B17, and B18 and AEO96 National Energy Modeling System runs TECHHM.D031496A, TECHMH.D031496A, TECHLM.D031496A, TECHMF.D031496A,

TECHHF.D040796B, and TECHLH.D040796A. 1994: Energy Information Administration, Annual Energy Outlook 1996, DOE/EIA-0383(96) (Washington, DC, January 1996). 
Figure 1. Fuel Prices, 2015

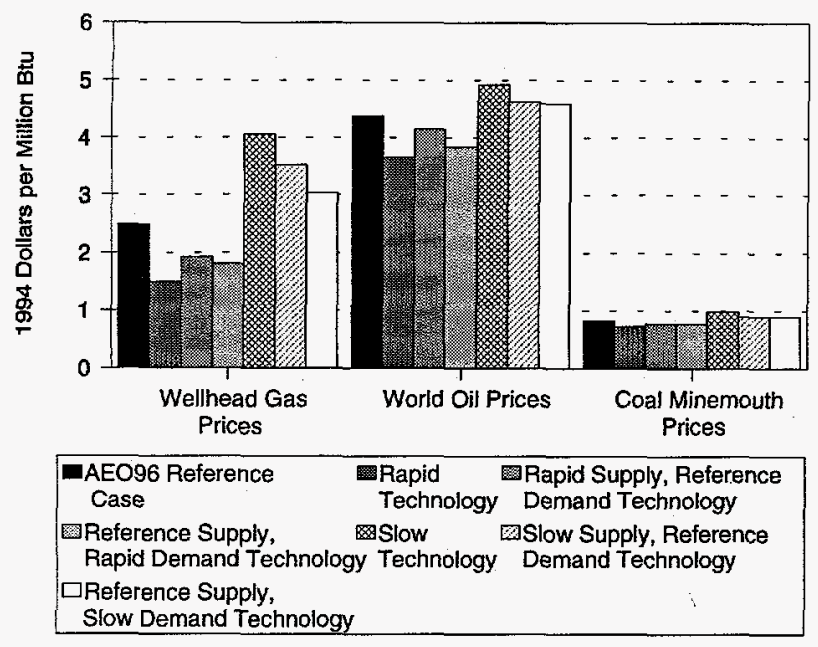

Source: Table B1 and AEO96 National Energy Modeling System runs TECHHM.D031496A, TECHMH.D031496A, TECHLM.D031496A, and TECHMF.D031496A.

gas price would have been $\$ 1.27$ per thousand cubic feet in 2015. The results are similar for coal and oil.

\section{Prices}

Using the AEO96 reference case international assumptions for oil markets, world oil prices were endogenously determined for the technology cases in response to changes in U.S. oil consumption and production patterns. Since the international oil market was assumed to remain unchanged from the AEO96 reference case, the penetration of domestic oil and gas technologies into the international market was not represented, and the feedback effect of lower oil prices on international oil demand was also not captured in these cases. This study focused on first-order effects of technological progress that is limited to U.S. markets.

World crude oil prices in 2015 vary from $\$ 21.17$ per barrel to $\$ 28.58$ per barrel, a 35-percent change between the slow and rapid technological progress cases. U.S. oil demand is expected to become a declining share of world oil consumption over the forecast period, and crude oil supply is expected to be plentiful internationally. These factors moderate the variation of oil prices to that of domestic coal price variations.

International gas markets have a limited influence on domestic U.S. gas prices. Natural gas does not have the international accessibility that international oil markets provide. Consequently, natural gas prices are more
Figure 2. Electricity Prices, 2015

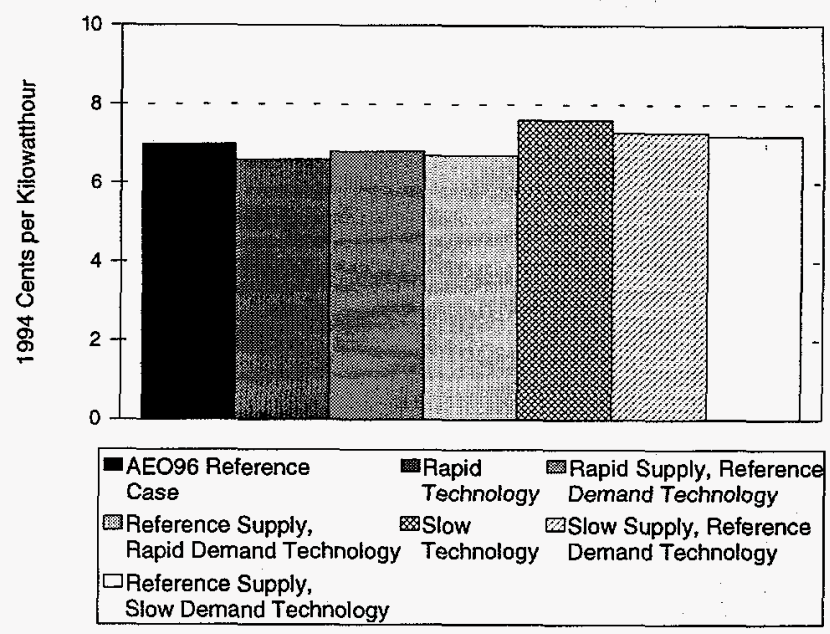

Source: Table B8 and AEO96. National Energy Modeling System runs TECHHM.D031496A, TECHMH.D031496A, TECHLM.D031496A, and TECHMF.D031496A.

sensitive to the quantities of economically recoverable resources available in the North American continent, and these, in turn, are dependent on the geology and the rate of technological progress. In 2015, natural gas wellhead prices are expected to range from $\$ 1.55$ per thousand cubic feet to $\$ 4.18$ per thousand cubic feet, a 170 -percent increase between the technology cases.

Coal prices range from $\$ 15.26$ per ton to $\$ 20.95$ per ton, a 37 -percent increase-slightly higher than the world oil price. Figure 2 illustrates the pattern of electricity prices which also follow the pattern in fossil fuel prices.

\section{Energy Consumption}

Total energy consumption in the rapid technological progress case is projected to be about 13 percent lower than the $A E O 96$ reference case because of the penetration of advanced technologies. When technological progress is assumed to be slower than the AEO96 reference case for both supply and demand technologies, fossil fuel prices in 2015 are projected to be substantially higher and total fossil fuel consumption is projected to be about 5 percent above the $A E 096$ reference case due largely to lower energy conversion efficiencies. Because the majority of energy-utilizing equipment turns over slowly, the impact of slow technological improvement on energy consumption during the forecast period is muted. Further, because energy represents less than 10 percent of GDP, increases in delivered energy costs have a mild but downward impact on GDP and consequently on energy service demand. 
With the rapid demand technology case held constant, the rapid supply technology case has only a slight impact on oil demand, despite the changed oil price. This occurs because of the way technological change and adoption were represented in the rapid demand technology case and because of the relative prices between oil and gas. Since transportation and industrial technology improvements and penetration were set to be autonomous, or independent of prices, in the rapid demand technology case, oil demand in these markets was virtually unchanged for any of the supply cases encountered. The other sectors, residential, commercial, and generation, saw lower oil prices but even lower gas prices, and fuel switching was largely discouraged. In 2015, when the rapid demand technology assumptions are combined with the rapid supply technology assumptions, the world oil price is about 6 percent lower and the wellhead gas price is about 16 percent lower then the case where rapid demand technology assumptions are combined with AEO96 reference case supply technology assumptions.

Coal and oil consumption in the slow technological progress case is projected to be 12 percent and 7 percent higher, respectively, in 2015 than the AEO96 reference case while natural gas consumption rises above and then declines to the AEO96 reference case in 2015. Natural gas consumption increases earlier in the projection period are due to the lower efficiencies in the menu of end-use technologies available to satisfy end-use demands. At the same time, the slower supply technological progress relative to the $A E O 96$ reference case drives up production costs and supply prices.

Because relative gas wellhead prices increase the most quickly in the slow technology case, natural gas market shares are reduced in end-use and electricity markets in the last decade of the forecast horizon. Higher natural gas consumption relative to the $A E O 96$ reference case through 2010, coupled with slower oil and gas technological progress, causes wellhead gas prices in the slow technological progress case to increase by 40 percent from 2010 to 2015.

Coal consumption increases primarily in the industrial and electricity generation sectors because of the assumed slowdown of efficiency improvements and some substitution for natural gas in generation. Oil consumption increases primarily in the transportation sector because of the assumed slowdown of efficiency improvements.

Relative to the assumptions in this study, technological progress for demand technologies has a greater potential influence in reducing oil imports and oil consumption than technological progress for supply (Figure 3). In these technology cases, rapid demand technological progress
Figure 3. Oil Consumption and Imports, 2015

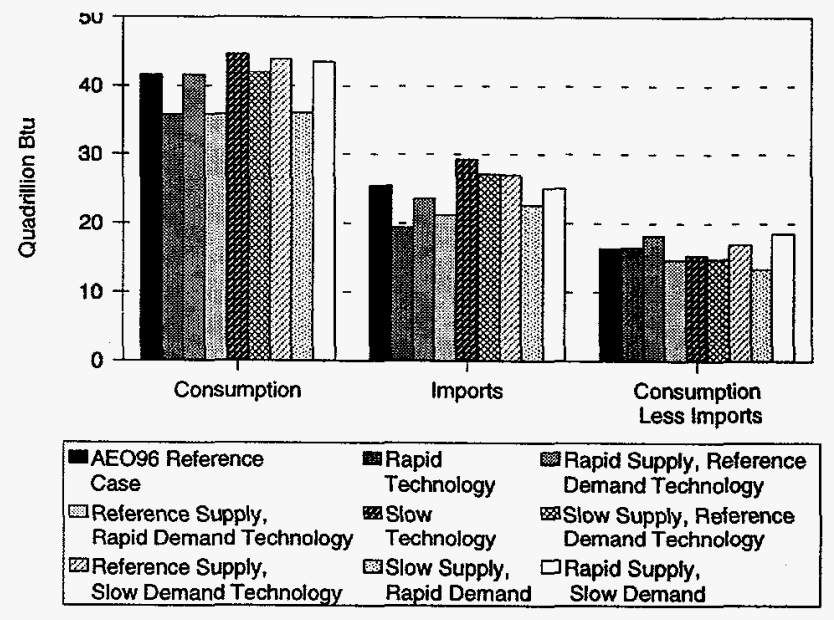

Source: Table B1 and AEO96 National Energy Modeling System runs TECHHM.D031496A, TECHMH.D031496A, TECHLM.D031496A, TECHMF.D031496A, TECHLH.D040796A, and TECHHF.D040796B.

reduces oil consumption by approximately 5.8 quadrillion British thermal units (Btu) in 2015 because the demand for oil is determined largely by transportation technology changes which are assumed to be independent of price. When rapid demand technological progress occurs with $A E O 96$ reference case supply technologies, oil imports are reduced by 4.3 quadrillion Btu. When both rapid supply and demand technological progress are assumed, oil imports are reduced by 6.0 quadrillion Btu representing additional displacement of oil imports by domestic oil production.

The rapid supply case coupled with reference case demand technology assumptions suggest that rapid supply technology assumptions-50-percent improvement over the AEO96 reference case-can reduce imports by about 1.9 quadrillion Btu in 2015 and increase the profitability of domestic oil and gas production. Import oil dependence is minimized when rapid demand technological progress is combined with rapid supply technological progress because demand technological progress reduces the overall demand and prices for delivered energy and rapid supply technological progress reduces exploration and production costs and prices and makes the domestic oil and gas industry more profitable.

Oil imports are projected to range from 19.4 quadrillion Btu in 2015 in the rapid technological progress case to 29.3 quadrillion Btu in the slow technological progress case. The highest domestic crude oil production in 2015 is projected to be 14.1 quadrillion Btu and results from rapid supply technological progress and slow demand technological progress assumptions. In the cases with 
rapid supply progress combined with reference or slow demand technological progress, the costs of domestic oil production are the lowest of the cases with correspondingly high domestic production; the assumed supply technological advances make more domestic oil resources economically recoverable and cheaper to produce, and world crude oil prices, $\$ 24.06$ per barrel and $\$ 25.41$ per barrel, respectively, provide domestic producers with adequate financial incentives to increase production in 2015 relative to the $A E O 96$ reference case.

Advances in supply technologies influence the tradeoff between oil imports and domestic crude oil production. Rapid supply technological progress assumptions result in lower production costs and increased domestic oil production in 2015 which displaces oil imports, all else being equal. Slow supply technological progress relative to the $A E O 96$ reference case increases domestic exploration and production costs, reduces the profitability of the domestic oil and gas industries, reduces domestic oil production, and increases oil imports. Domestic oil consumption is determined primarily by transportation demand and the demand technological progress assumptions, regardless of which supply technological progress assumptions are used. For example, domestic oil consumption hovers around 42 quadrillion Btu whenever the AEO96 reference case demand technology assumptions are used. When the rapid demand technological progress assumptions are used, U.S. oil consumption in 2015 is projected to fall to about 36 quadrillion Btu, regardless of which supply technological progress assumptions are used.

The rate of demand technological progress primarily affects consumption for fuels and fuel prices while the rate of supply technological progress primarily affects fuel prices and domestic production. Consequently, fuel consumption is at its greatest when lower end-use efficiencies, leading to higher fuel consumption, are coupled with higher supply efficiencies, lower prices and greater domestic supply. Similarly, fuel consumption, both in the aggregate and for each fuel, is at its lowest when end-use efficiencies are their highest. However, in the slow technology case, slow supply technological progress increases the price of natural gas faster than either coal, which is far more abundant domestically than natural gas, or oil, whose prices are moderated by the international markets. Consequently, natural gas wellhead prices rise to $\$ 4.18$ per thousand cubic feet in 2015 , and natural gas loses market share primarily to coal for generation and to electricity at end-use markets.

Natural gas consumption in 2015 ranges from 25.4 quadrillion Btu in the rapid technological progress case to
29.4 quadrillion Btu in the slow technological progress case, corresponding to natural gas prices from $\$ 1.55$ per thousand cubic feet to $\$ 4.18$ per thousand cubic feet, respectively (Figure 1). The greatest gas consumption in 2015-31.3 quadrillion Btu-is projected to occur, however, in the case that uses rapid supply technological progress assumptions combined with slow demand technological progress assumptions (Table 1). The lowest gas consumption occurs with rapid demand technological progress assumptions. The rapid supply/slow demand technological progress case is equivalent to a case in which fuel supply costs are low and consumers use lowest capital costs as the primary criterion to make equipment and efficiency choices. In this case, gas prices-\$2.17 per thousand cubic feet-are projected to be much lower than the price of $\$ 2.57$ per thousand cubic feet in the AEO96 reference case in 2015; consequently, natural gas and total energy consumption is projected to be correspondingly higher.

\section{Carbon Emissions}

Carbon emissions are directly correlated with fossil fuel consumption. The AEO96 reference case projects 1,735 million metric tons of anthropogenically-generated carbon emissions in the United States in 2015, an average of 5.6 tons per person. Under the rapid supply and demand technological progress case, carbon emissions are projected to decline to about 1,540 million metric tons, 5.0 tons per person, while in the slow technological progress case, carbon emissions are projected to rise to 1,863 million metric tons, 6.0 tons per person. None of these cases achieve the 1990 emission levels of 1,335 million metric tons of carbon and 5.4 tons per person.

Although none of the cases examined achieve carbon stabilization despite the favorable assumptions made in the rapid technology case, the results suggest that R\&D investments that succeed in developing low-cost, highefficiency end-use technologies and facilitate market acceptance of them could play a significant role in efforts to moderate carbon emissions in the United States. R\&D programs that improve the availability and market acceptance of cost-efficient transportation technologies coupled with successful oil and gas supply $R \& D$ programs could have a significant impact on reducing imported oil dependence if these programs were successful.

\section{Price-Induced GDP Changes}

Due to fuel price changes, the GDP ranges from $\$ 8,072$ billion (1987 dollars) to $\$ 8,158$ billion, a 1-percent or $\$ 86$ billion change in the GDP in 2015. The price-induced GDP losses/gains of the slow/rapid technological progress 
cases are approximately symmetrically distributed around the reference case.

\section{Electricity Generation Market}

In the electric power industry, conventional and advanced generation technologies compete whenever new generation capacity is required. Conventional capacity is mature and is characterized by having stable construction and operating costs. The operating and cost characteristics of advanced technologies are typically uncertain and dynamic. The rates of technological improvements affect the relative economics of conventional and advanced technologies, which impact capacity expansion and operating decisions in the longer-term.

Relative to the $A E O 96$ reference case, the slow and rapid technology cases examine the impact of alternative assumptions about technological optimism and learning on generation technology costs and efficiency improvements and the consequent impact on the market penetration of generation technologies. The alternative technology cases examined in this study do not attempt to evaluate Federal energy goals or programs. Cost reductions and efficiency improvements used to define the rapid technology case should not be interpreted as limits to technological cost and performance. The future success of applied research efforts cannot be known. Significantly greater improvement in cost reductions and efficiency improvements may be attainable through applied research programs. Technological optimism factors are inputs to the NEMS electricity module and reflect the degree of design and cost uncertainties for each new adyanced technology. Such factors are developed from engineering estimates that reflect how much of the design is new and, hence, may need initial minor redesign or "fine-tuning." Typically, the costs associated with the first-of-a-kind-plants include the costs of the first few nonoptimally constructed plants and the lack of manufacturing experience. Consistent with historical evidence, once the manufacturer has gained experience and finetuned the design, manufacturing experience, or learning by doing, affects economies of scale and reduces the cost of units built later. The degree of technological optimism associated with costs for advanced technologies depends on the stage of development and the complexity of design. Learning effects are also assumed for operating efficiencies. The final mature-technology capital costs and operating efficiencies are the same for all cases, but the technology cases assume that the improvements occur differently.
The assumptions for the technological optimism and learning rates are the same as the corresponding assumptions used in the technological optimism cases discussed in the AEO96. As in the AEO96 reference case, it is assumed that no new nuclear plants, either conventional or advanced, will be built. Advanced fossil technology options include coal-fired integrated gasified combined cycle (IGCC), combined cycle, combustion turbine, and fuel cells. Geothermal, biomass, solar thermal, and solar photovoltaics are considered to be advanced renewable technologies.

Compared to the AEO96 reference case, the slow technological progress case incorporates more pessimistic assumptions for the initial first-of-a-kind costs and for the rate that costs decline thereafter due to learning. The slow technological progress case also requires the completion of more units before the technological optimism adjustment is eliminated and the reductions due to learning are fully realized. Further, improvements in operating efficiencies are also assumed to occur more slowly than in the AEO96 reference case. In the AEO96 reference case, operating efficiencies are assumed to increase until 2010 and then remain constant. In the slow technological progress case, the same efficiency improvements are not achieved until 2015. As a result of the assumptions for capital costs and operating efficiencies in the slow technological progress case, advanced technologies are less competitive with conventional generation equipment than in the AEO96 reference case.

Compared to the AEO96 reference case, the rapid technological progress case incorporates more optimistic assumptions for the technological optimism and learning that result in lower capital costs for the initial units of advanced technologies, completion of fewer units before the technological optimism adjustment is eliminated, and faster cost reductions due to learning. Improvements in operating efficiencies are assumed to occur by 2005 , which is five years sooner than in the AEO96 reference case. As a result of the assumptions for capital costs and operating efficiency improvements in the rapid technological progress case, advanced technologies are more competitive with conventional capacity types than in the AEO96 reference case.

The rates 0 : technological progress in the electric power industry affect capacity expansion decisions, which in turn impact fuel consumption and delivered fuel prices. Furthermore, planning and operating decisions are affected by the demand for electricity, which varies across the cases as a result of the technological improvement rates in the end-use sectors. 


\section{Slow Technological Progress}

In 2015, the demand for electricity in the slow technological progress case is about 4 percent higher than in the AEO96 reference case due to the lower efficiencies assumed in end-use sectors. Consequently, the need for new capacity after 1996 beyond those projects already planned is about 46 gigawatts higher than in the AEO96 reference case (Table 2). Because of technological optimism and learning factors that yield higher actual costs, advanced technologies account for 39 percent of the total unplanned additions, compared to 49 percent in the AEO96 reference case.

Most of the incremental capacity requirements in the slow technological progress case are met by conventional combustion turbine, combined cycle, and coal-fired facilities. However, as natural gas becomes increasingly more expensive than coal and oil in the last decade of the projections, coal capacity additions are increasingly favored over natural gas units. Although the share of advanced fossil technologies is lower than the AEO96 reference case, slightly more fossil-fueled capacity is built as a result of the higher total capacity requirement. In contrast, both the share and amount of renewable additions are lower in the slow technological progress case. Most renewable technologies have higher capital costs and lower operating costs than conventional fossilfired plants so they are more sensitive to changes in the learning and technological optimism factors. Renewable technologies, like advanced fossil technologies, face larger first-of-a-kind costs and slower learning-by-doing in the slow technology case than in the reference case. Consequently, renewable technologies, like other advanced technologies, take longer to become economic and penetrate the generation market.

Compared to the $A E O 96$ reference case, coal consumption in the electricity generation market in 2015 is 6 percent higher in the slow technological progress case due to the higher demand for electricity and the corresponding increase in coal-fired generating capacity. Although there is no increase in oil-fired generating capacity in the slow technological progress case, oil consumption increases by 46 percent primarily due to switching from natural gas to oil in conventional dual-fired steam plants. This fuel switching occurs because of changes in the relative fuel prices between the two cases. In 2015, the delivered prices of oil and natural gas are 11 percent and 55 percent higher, respectively, in the slow technological progress case than in the AEO96 reference case. The higher fuel prices in the slow technological progress case are largely due to the lower efficiencies assumed in the end-use sectors, which result in higher fuel consumption than in the AEO96 reference case. However, natural gas use in 2015 is only 3 percent lower in the slow technological progress case because the switching from natural gas to oil is partially offset by increased generation from new gas-fired facilities, which are built as a result of the higher demand for electricity.

\section{Rapid Technological Progress}

In 2015, the demand for electricity in the rapid technological progress case is about 12 percent lower than in the AEO96 reference case due to the higher efficiencies assumed in the end-use sectors. Consequently, the need for new capacity beyond those projects already planned is about 93 gigawatts lower than the AEO96 reference case (Table 2). In spite of lower initial capital costs and faster learning, advanced technologies account for only 35 percent of the total unplanned additions, compared to 49 percent in the AEO96 reference case. The lower share of capacity additions for advanced technologies occurs because the lower total capacity requirements reduce opportunities for penetration of advanced technologies, which delays cost reductions due to the technological optimism and learning-by-doing.

Compared to the AEO96 reference case, generation market fuel use patterns in the rapid technological progress case follow the corresponding trends in capacity additions. That is, consumption of coal and natural gas is lower due to fewer capacity additions of conventional and advanced coal- and gas-fired capacity. Lower fuel consumption in the end-use demand sectors results in fossil fuel prices that are considerably lower in the rapid technological progress case than in the $A E O 96$ reference case.

\section{Residential Energy Markets}

In the slow technological progress case, the menu of technologies available to the residential market is restricted to the technologies available in 1995. Building shell efficiencies for new and existing buildings are assumed to remain unchanged with respect to their status in 1995. Fuel prices may affect the choice of end-use equipment purchases and efficiencies over the forecast horizon. The menu of technologies, however, is that which was available in 1995. Consumers' decision rules are those specified in the AEO96 reference case.

In the rapid technological progress case, the menu of available end-use technologies is exactly the same as for the AEO96 reference case. However, all consumer discount rates relating to fuel and equipment choices are set at 7 percent real. Discount rates in the AEO96 reference case 
Table 2. Electricity Results, 2015

\begin{tabular}{|c|c|c|c|}
\hline Electricity Indicators & $\begin{array}{l}\text { Slow Technological } \\
\text { Progress Case }\end{array}$ & $\begin{array}{c}\text { AEO96 } \\
\text { Reference Case } \\
\end{array}$ & $\begin{array}{c}\text { Rapid Technological } \\
\text { Progress Case }\end{array}$ \\
\hline Electricity Sales (billion kilowatthours) & 4,029 & 3,889 & 3,407 \\
\hline \multicolumn{4}{|l|}{$\begin{array}{l}\text { Cumulative Unplanned Capacity Additions } \\
\text { (gigawatts) }\end{array}$} \\
\hline \multicolumn{4}{|l|}{ Conventional Technologies } \\
\hline Coal....... & 23.0 & 7.5 & 1.0 \\
\hline Combined Cycle & 14.8 & 1.9 & 0.8 \\
\hline Combustion Turbine ...... & 95.1 & 77.9 & 48.8 \\
\hline Subtotal-Conventional & 132.9 & 87.3 & 50.5 \\
\hline \multicolumn{4}{|l|}{ Advanced Technologies } \\
\hline \multicolumn{4}{|l|}{ Fossil } \\
\hline Coal $\ldots \ldots \ldots \ldots \ldots \ldots \ldots \ldots, \ldots$ & 2.3 & 1.6 & 1.0 \\
\hline Combined Cycle ...... & 66.2 & 64.2 & 14.5 \\
\hline Combustion Turbine $\ldots \ldots \ldots \ldots \ldots$ & 2.0 & 2.3 & 2.4 \\
\hline Fuel Cells ..................... & 3.5 & 2.3 & 1.9 \\
\hline Subtotal-Fossil ..... & 74.0 & 70.4 & 19.7 \\
\hline \multicolumn{4}{|l|}{ Renewable } \\
\hline Geothermal .................... & 1.9 & 1.4 & 0.4 \\
\hline Biomass ............ & 0.2 & 0.9 & 0.4 \\
\hline Wind $\ldots \ldots \ldots \ldots \ldots \ldots \ldots \ldots \ldots \ldots$ & 6.5 & 8.6 & 4.6 \\
\hline Solar Thermal $\ldots \ldots \ldots \ldots \ldots \ldots$ & 0.9 & 1.4 & 1.0 \\
\hline Solar Photovoltaics $\ldots \ldots \ldots \ldots \ldots \ldots$. & 0.3 & 0.3 & 0.7 \\
\hline Subtotal-Renewable ... & 9.8 & 12.5 & 7.1 \\
\hline Subtotal-Advanced & 83.7 & 82.9 & 26.8 \\
\hline Total-Unplanned Capacity Additions ..... & 216.6 & 170.2 & 77.3 \\
\hline \multicolumn{4}{|l|}{ Fuel Consumption (quadrillion Btu) } \\
\hline Coal .............. & 21.8 & 20.6 & 19.5 \\
\hline Natural Gas $\ldots \ldots \ldots \ldots \ldots \ldots \ldots \ldots$ & 7.3 & 7.5 & 5.3 \\
\hline 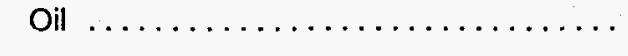 & 1.6 & 1.1 & 1.0 \\
\hline \multicolumn{4}{|l|}{ Fuel Prices (1994 dollars per million Btu) } \\
\hline Coal & 1.44 & 1.28 & 1.17 \\
\hline Natural Gas...$\ldots \ldots \ldots$. & 4.58 & 2.95 & 1.79 \\
\hline 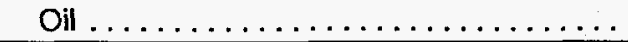 & 4.86 & 4.38 & 3.68 \\
\hline
\end{tabular}

AEO = Annual Energy Outlook.

Btu $=$ British thermal units.

Source: Tables B2, B3, and B8 and AEO96 National Energy Modeling System runs TECH01.D031396A, AEO96B.D101995C, and TECH04.D031396B.

range from 20 percent to over 100 percent, depending on the technology (Table 3). The shell efficiency for the 1993 existing housing stock is assumed to increase to a level consistent with 1994 new construction by 2015. Relative to the $A E O 96$ reference case, the rapid technological progress case assumptions yield shell efficiencies for existing houses that are 6 and 3 percent higher for heating and cooling, respectively. 
Table 3. Consumer Discount Rate Assumptions by Appliance (Percent)

\begin{tabular}{|c|c|c|c|}
\hline Appliance Type & $\begin{array}{c}\text { Slow Technological } \\
\text { Progress Case }\end{array}$ & $\begin{array}{c}\text { AEO96 } \\
\text { Reference Case }\end{array}$ & $\begin{array}{l}\text { Rapid Technological } \\
\text { Progress Case }\end{array}$ \\
\hline Heating Fuel Type for New Construction . . . . . . & 20 & 20 & 7 \\
\hline Heating $\ldots \ldots \ldots \ldots \ldots \ldots \ldots \ldots \ldots$ & 30 & 30 & 7 \\
\hline Cooling $\ldots \ldots \ldots \ldots \ldots \ldots \ldots \ldots$ & 33 & 33 & 7 \\
\hline Electric Water Heater . . . . & 111 & 111 & 7 \\
\hline Distillate Water Heater & 50 & 50 & 7 \\
\hline Natural Gas Water Heater . . . . . . . . . . . . & 63 & 63 & 7 \\
\hline Electric Clothes Dryer & 120 & 120 & 7 \\
\hline Refrigerator $\ldots \ldots \ldots$ & 69 & 69 & 7 \\
\hline Freezer $\ldots \ldots \ldots \ldots \ldots \ldots \ldots \ldots \ldots \ldots$ & 91 & 91 & 7 \\
\hline
\end{tabular}

AEO = Annual Energy Outlook.

Source: Energy Information Administration, Office of Integrated Analysis and Forecasting.

The shell efficiency for new construction improves at twice the rate of the AEO96 reference case through 2015. All else being equal, e.g., fuel prices, this implies that new buildings built in 2015 would be between 15 and 20 percent more efficient in terms of heating and cooling shell efficiencies in the rapid technological progress case than new buildings in the AEO96 reference case. However, all is not equal because prices in the rapid technology case fall in response to lower demand, thereby reducing priceinduced shell efficiency gains by about 50 percent. New technologies made available in the AEO96 reference case were used because these represent significant advances in efficiency improvements over time at declining capital costs as Table 4 illustrates.

Table 4 demonstrates the importance of consumer behavior, imbedded in their discount rates, in determining the market share of more efficient technologies. The technologies represented in Table 4 are the most efficient models available in the appropriate year. Relative to the AEO96 reference case, the rapid technological progress case yields greater market shares for these efficient technologies because of lower consumer discount rates. Table 3 presents the consumer discount rates assumed for the various technologies. In Table 3, efficiency refers to the discount rate associated with choosing the efficiency level of the appliance, as opposed to the fuel choice among different technologies, e.g., gas versus electric. All rows in the table refer to efficiency choice except for the first, which is the fuel choice for new construction.

Tables 3 and 4 demonstrate the effect of lowering the consumer discount rates and thus lengthening payback periods, by comparing the market share the technology receives under each case. For example, in the rapid technological progress case, the electric air-source hot water heater gains 71 percent market share over the AEO96 reference case by 2015 due to a decrease in the consumer discount rate from 111 percent to 7 percent. The other examples provided in Tables 3 and 4 are comparable in terms of their relationship between market share and consumer discount rate assumptions.

\section{Slow Technological Progress}

The slow technological progress case, in which no technological advances occur after 1995, requires 5 percent more natural gas and electricity by 2015 than the AEO96 reference case. However, natural gas prices rise 27 percent relative to $A E O 96$ by 2015 , causing a partial shift away from natural gas appliances toward electric and distillate, dampening the potential increase in natural gas consumption had prices not increased as dramatically. Figure 4 shows the sharp increase in residential natural gas prices relative to the AEO96 reference case and the less variable electricity prices.

\section{Rapid Technological Progress}

In the rapid technological progress case, fuel consumption and prices decline as a result of increasing end-use energy efficiency, relative to $A E O 96$. Electricity consumption declines the most by 2015 because electric-powered end uses have much more potential for energy efficiency gains and end-use prices are expected to be relatively stable. Heat pumps exhibit an enormous gain in stock efficiency 
Table 4. Residential Technology and Building Efficiency Indices, 2015 $(1995=1.00)$

\begin{tabular}{|c|c|c|c|c|}
\hline Best Technology Available & 1995 & $\begin{array}{c}\text { Slow Technological } \\
\text { Progress Case }\end{array}$ & $\begin{array}{c}\text { AEO96 } \\
\text { Reference Case }\end{array}$ & $\begin{array}{l}\text { Rapid Technological } \\
\text { Progress Case }\end{array}$ \\
\hline \multicolumn{5}{|l|}{ Shell Efficiency } \\
\hline Existing & 1.00 & 1.00 & 1.03 & 1.09 \\
\hline New. & 1.10 & 1.10 & 1.36 & 1.48 \\
\hline \multicolumn{5}{|l|}{ Natural Gas Furnace } \\
\hline Cost $\ldots \ldots \ldots \ldots$ & 1.00 & 1.00 & 0.98 & 0.98 \\
\hline Efficiency.......... & 1.00 & 1.00 & 1.07 & 1.07 \\
\hline Market Share (percent) $\ldots \ldots \ldots \ldots$ & 4 & 4 & 35 & 69 \\
\hline \multicolumn{5}{|l|}{ Air-Source Heat Pump } \\
\hline Cost $\ldots \ldots \ldots \ldots \ldots \ldots, \ldots, \ldots$ & 1.00 & 1.00 & 0.71 & 0.71 \\
\hline Efficiency $\ldots \ldots \ldots \ldots \ldots \ldots \ldots$ & 1.00 & 1.00 & 1.13 & 1.13 \\
\hline Market Share (percent) & 2 & 2 & 40 & 54 \\
\hline \multicolumn{5}{|l|}{ Electric Air Source Hot Water Heater } \\
\hline Cost $\ldots \ldots \ldots \ldots$ & 1.00 & 1.00 & 0.56 & 0.56 \\
\hline Efficiency ..... & 1.00 & 1.00 & 1.64 & 1.64 \\
\hline Market Share (percent) & 0 & 0 & 2 & 73 \\
\hline
\end{tabular}

$\mathrm{AEO}=$ Annual Energy Outlook.

Source: AEO96 National Energy Modeling System runs TECH01.D031396A, AEO96B.D101995C, and TECH04.D031396B.

Figure 4. Residential Energy Consumption, Prices, and Efficiency, 2015

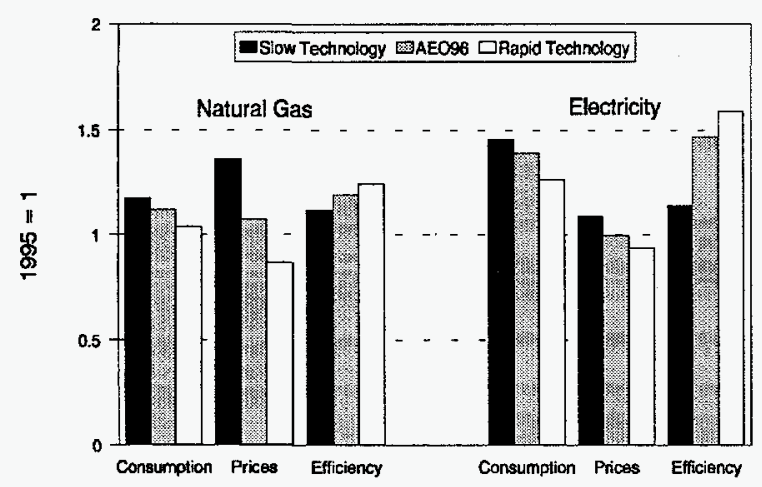

Source: Tables B2 and B3 and AEO96 National Energy Modeling System runs TECH01.D031396A, AEO96B.D101995C, and TECH04.D031396B.

in both the AEO96 reference case and the rapid technological progress case. With discount rates lowered from 20 percent to 7 percent, electric heat pump stock efficiency increases more than 50 percent over 1995 levels, causing a 26 -percent decline in space cooling intensity by 2015 , relative to 1995 . Figure 5 illustrates residential sector energy intensity.

\section{Commercial Energy Markets}

The assumptions for the slow technological progress case are identical to the assumptions for the commercial sector 1995 technology case defined for AEO96. The same end-use technologies are assumed to be available in both the rapid technological progress and the AEO96 reference case; however, the distribution of discount rates relating to equipment choice was adjusted in the rapid technological progress case to represent a greater willingness to invest for future energy savings. Discount rates for this case are defined such that half of the commercial sector consumers will invest at the risk-free interest rate, 25 percent of commercial consumers will invest at the risk-free rate plus 10 percent, and 25 percent of commercial consumers will invest at the risk-free rate plus 20 percent. For equipment expected to last 15 years, the resulting time required by consumers to recover their investment would be approximately eleven, six, and four years respectively.

Discount rates in the $A E 096$ reference case range from the risk-free rate plus 13.6 percent, roughly a five-year payback period, to over 1,000 percent, with 33 percent of commercial consumers at the highest discount rate, thus assuming to base equipment purchases solely on capital costs. By 2015, the shell efficiency for existing commercial building stock is assumed to increase to a level consistent 
with 1995 new construction, about 16 percent more efficient than the average existing building shell in 1992. Existing commercial building shell efficiency in the AEO96 reference case is assumed to improve 5 percent over the 1992 average by the year 2015 .

Table 5 illustrates the effects that commercial consumer discount rates and availability have on the market share of efficient technologies. The technologies represented in Table 5 are the most efficient models available in the appropriate year. The most efficient technologies gain a greater percentage of the market in the rapid technological progress case relative to the $A E O 96$ reference case as a result of lower discount rates. The 92-percent market share for the gas-fired chiller in the slow technological progress case indicates economic conditions in which the most efficient technology available can succeed in a market employing current choice patterns.

\section{Slow Technological Progress}

No efficiency advances in either end-use equipment or existing building shells are projected after 1995 in the slow technological progress case. For this case, the commercial sector is projected to consume 2 percent more electricity and 3 percent more distillate fuel oil by 2015 as compared to the $A E 096$ reference case.

Given these assumptions, delivered electricity prices in 2015 to the commercial sector are the least affected while delivered natural gas prices are the most affected, a 29-
Figure 5. Residential Energy Intensity, 1980-2015

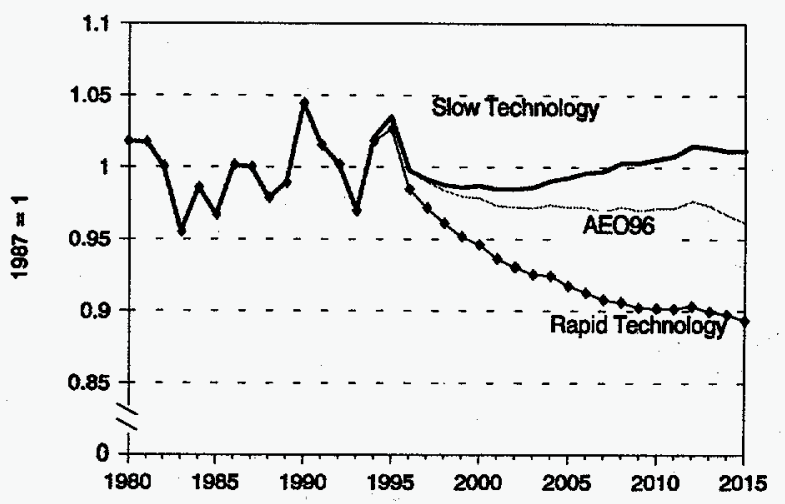

Source: History: Residential energy intensity is calculated using consumption, households, and weather data. The historical data are as follows: Consumption: Energy Information Administration, State Energy Data Report 1991, DOE/EIA0214(91) (Washington, DC, May 1993). Weather: Energy Information Administration, Annual Energy Review 1994, DOE/EIA-0384(94) (Washington, DC, July 1995). Households: DRI/McGraw-Hill, US Quarterly Model US96A, February 1996. Projections: AEO96 National Energy Modeling System runs TECH01.D031396A, AEO96B.D101995C, and TECH04.D031396B.

percent increase, relative to the $A E O 96$ reference case. Consequently, natural gas consumption declines about 2 percent while electricity and oil consumption increase relative to the AEO96 reference case (Figure 6).

Table 5. Commercial Technology Indices, 2015 $(1995=1.00)$

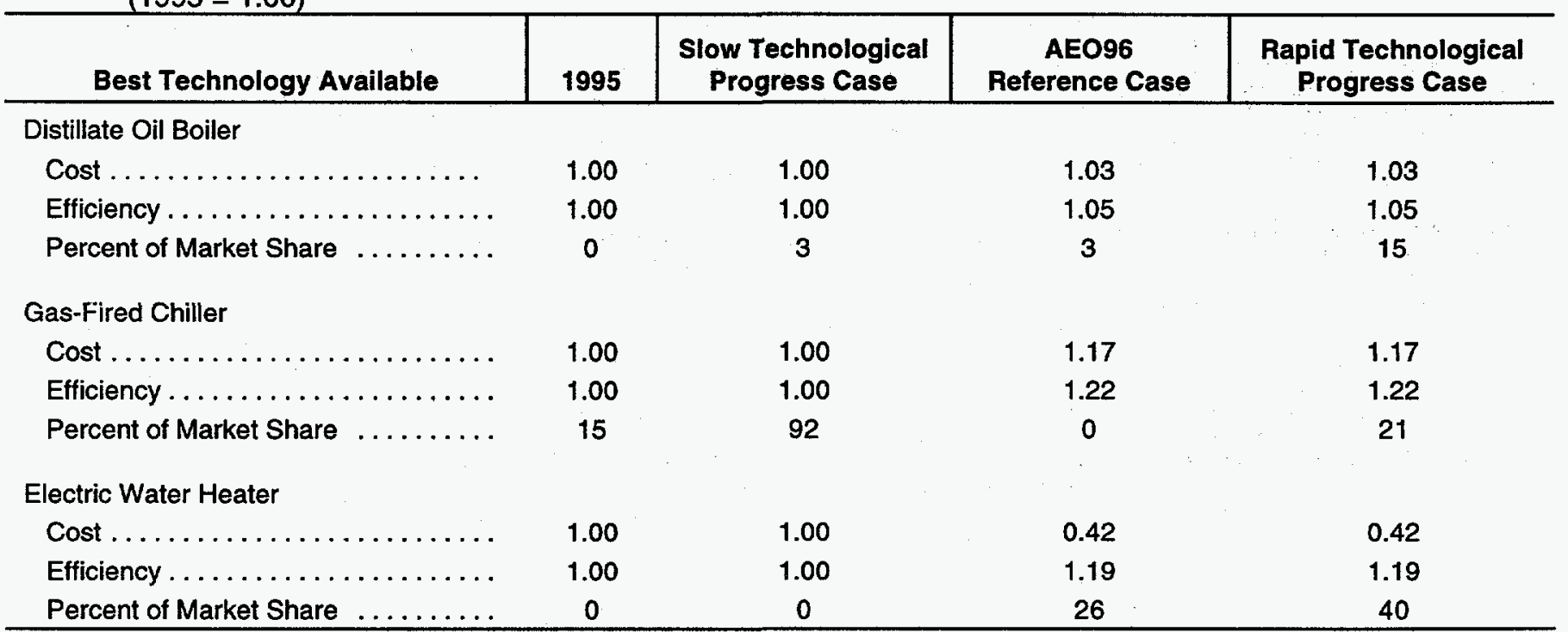

AEO = Annual Energy Outlook.

Source: AEO96 National Energy Modeling System runs TECH01.D031396A, AEO96B.D101995C, and TECH04.D031396B. 
Figure 6. Commercial Energy Consumption, Prices, and Efficiency, 2015

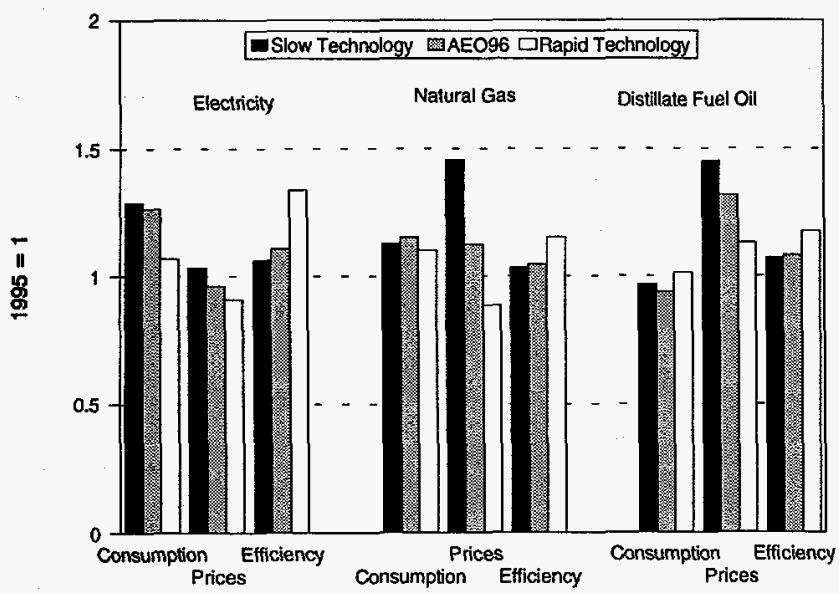

Source: Tables B2 and B3 and AEO96 National Energy Modeling System runs TECH01.D031396A, AEO96B.D101995C, and TECH04.D031396B.

\section{Rapid Technological Progress}

Delivered energy to the commercial sector in the rapid technological progress case fell by about 10 percent in 2015 relative to the AEO96 reference case due to the significant availability of new technologies. Nevertheless, the share of natural gas in the total delivered energy to the commercial sector actually rises about 2 percent above the $A E O 96$ reference case while the share of electricity actually declines by about 3.5 percent.

The commercial discount rates assumed for the rapid technological progress case contribute to increasing enduse energy efficiency which in turn results in declines in both fuel prices and energy intensity, relative to AEO96.

Consumption of distillate fuel oil actually increases in this case as commercial consumers switch from electricity to oil, and, to some extent, natural gas-powered space heating equipment. Relative prices again play an important role in determining fuei shares, with oil and gas prices showing a greater decline than electricity prices when compared to AEO96.

\section{Transportation Energy Markets}

The slow technological progress case assumes that energy efficiencies for all modes of transportation remain at 1995 levels throughout the forecast period. The rapid progress case assumes that fuel efficiency improvement rates continue throughout the forecast period at the historical growth rates of the 1970 to 1990 period. If the programmatic goals of the advanced vehicle programs were met by 2007 and other performance characteristics, such as horsepower, remain acceptable to consumers, new car efficiency could significantly increase above the 40 miles per gallon in 2015 indicated in the rapid technology case.

\section{Slow Technological Progress}

Although travel demand, e.g., vehicle miles traveled, declines in response to higher fuel prices (Tables 6 and 7), the lack of efficiency improvement in the slow technological progress case results in an increase in fuel consumption of 7 percent in 2015 relative to the AEO96 reference case.

Figure 7 shows that the light-duty vehicles, which accounted for more than 55 percent of total energy use in transportation by 2015 , consume 11 percent more than the $A E 096$ reference case as travel demand decreases by 1.2 percent. Freight trucks primarily use distillate fuel and consume 5 percent more fuel in 2015 when freight transport declines by 0.6 percent. The constant 1995 aircraft fuel efficiency results in an increase in jet fuel consumption by 4 percent despite a decline of 1.4 percent in air travel. Unlike other modes, the efficiency in shipping does not change from the AEO96 reference case (Table 6); therefore, residual fuel consumption decreases by 1.3 percent in response to a 7.2 percent decline in freight shipping. As a result, energy use in rail, marine, and other transportation modes decreases by 1 percent in 2015. Figure 8 illustrates the new car miles per gallon paths for each of the three cases.

\section{Rapid Technological Progress}

Higher efficiency improvements relative to the AEO96 reference case are projected to produce significant energy savings for all modes of transportation despite slightly higher travel demand in all modes. The total fuel consumption is projected to decline by 11 percent in 2015. Most of the fuel savings come from light-duty vehicles and freight trucks, which result in a decrease in consumption of 9.5 percent and 20 percent, respectively. Efficiency improvements in new aircraft, particularly the Boeing 777 jumbo jets, combined with a decline in military use of jet fuel, account for the 4-percent fuel savings. The assumed fuel efficiency improvements result in energy savings and more than offset the rise in travel demand. Rail and shipping efficiencies in the rapid technology case improve slightly faster than the rate of improvement for new cars because, by assumption, the rapid technology case assumes that the annual efficiency improvement rates by mode that occurred in the last twenty years will continue through the forecast period. 
Table 6. Travel Demand and Efficiency, 2015

\begin{tabular}{|c|c|c|c|}
\hline Travel Modes & $\begin{array}{l}\text { Slow Technological } \\
\text { Progress Case }\end{array}$ & $\begin{array}{c}\text { AE096 } \\
\text { Reference Case }\end{array}$ & $\begin{array}{l}\text { Rapid Technological } \\
\text { Progress Case }\end{array}$ \\
\hline & \multicolumn{3}{|c|}{ Transportation Demand by Mode (Billions) } \\
\hline Light Duty Vehicles (vehicle-miles traveled) & 2,742 & 2,774 & 2,807 \\
\hline Freight Trucks (vehicle-miles traveled) & 486 & 489 & 493 \\
\hline \multirow[t]{2}{*}{ Marine (ton-miles traveled) } & 918 & 989 & 1,160 \\
\hline & \multicolumn{3}{|c|}{ Fuel Efficiencies } \\
\hline New Car (miles/gallon) & 27.5 & 33.1 & 40.2 \\
\hline Aircraft (seat-miles/gallon) & 57.2 & 60.5 & 65.6 \\
\hline Rail (ton-miles/thousand Btu) & 2.9 & 3.1 & 4.0 \\
\hline Shipping (ton-miles/thousand Btu) $\ldots \ldots \ldots$ & 2.6 & 2.6 & 3.6 \\
\hline
\end{tabular}

$\mathrm{AEO}=$ Annual Energy Outlook.

Source: Table B7.

Table 7. Fuel Prices, 2015

(1994 Dollars per Million Btu)

\begin{tabular}{c|c|c|c}
\hline Fuel Prices & $\begin{array}{c}\text { Slow Technological } \\
\text { Progress Case }\end{array}$ & $\begin{array}{c}\text { AEO96 } \\
\text { Reference Case }\end{array}$ & $\begin{array}{c}\text { Rapid Technological } \\
\text { Progress Case }\end{array}$ \\
\hline Motor Gasoline $\ldots \ldots \ldots \ldots \ldots \ldots \ldots \ldots \ldots \ldots$ & 11.85 & 10.86 & 9.42 \\
Distillate $\ldots \ldots \ldots \ldots \ldots \ldots \ldots \ldots \ldots \ldots \ldots$ & 9.81 & 9.21 & 8.23 \\
Jet Fuel $\ldots \ldots \ldots \ldots \ldots \ldots \ldots \ldots \ldots \ldots \ldots$ & 7.13 & 6.36 & 5.40 \\
Residual $\ldots \ldots \ldots \ldots \ldots \ldots \ldots \ldots \ldots$ & 4.23 & 3.72 & 3.06 \\
\hline
\end{tabular}

AEO = Annual Energy Outlook.

Source: Table B3.

\section{Industrial Energy Markets}

The slow technological progress case in the industrial sector examines two combinations of technology assumptions about energy supply and demand: (a) that industry will not install new equipment that exceeds the 1995 level of efficiency, although new equipment will be installed to replace retirements and to meet growing demand for industrial output, and (b) industrial sector energy consumption will fall somewhat in response to higher energy prices resulting from reduced efficiency of energy supply technologies over the forecast horizon.
Over the 1970 to 1990 period, industrial energy intensity fell by 1.9 percent annually. This was due to energy conservation and changes in the composition of industrial output. The changing composition of industrial output accounted for about 40 percent of the intensity decline. The rapid technological progress case approximates the 1.9percent annual intensity decline from 1995 through 2015 solely through technological change. This is almost twice the rate of intensity decline in the AEO96 reference case of 1.0 percent over the same period. Since the composition of industrial output is unchanged across these technology cases, the 1-percent difference is due strictly to increased 
Figure 7. Fuel Consumption by Transportation Modes, 2015

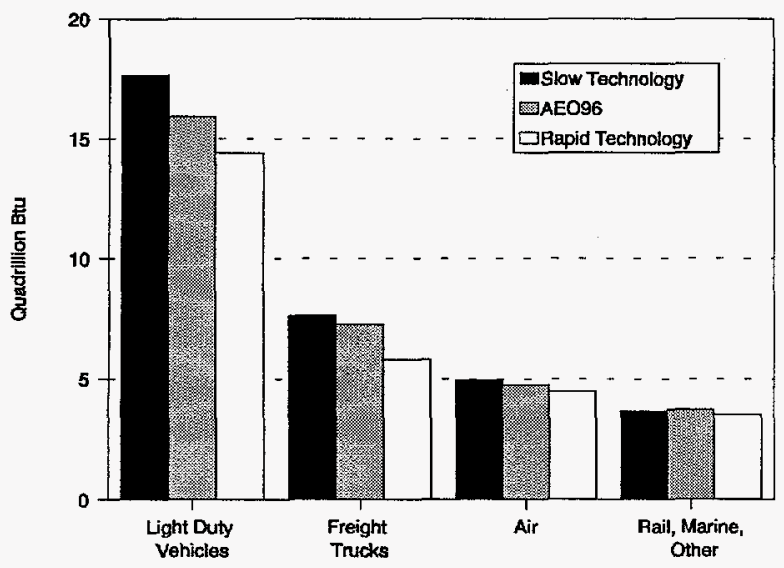

Source: Table B7.

conservation. The intensity fall is partially offset by the rebound effect caused by lower energy prices, resulting from increased efficiency in supply technology over the forecast period.

The annual rate of energy intensity decline ranges from 0.7 percent in the slow technological progress case to 1.9 percent in the rapid technological progress case (Figure 9). Energy consumption in the energy-intensive manufacturing industries experiences the most significant consumption changes due to technology assumptions. The price of natural gas to the industrial sector exhibits the largest response to the assumptions. Delivered natural gas prices increase by 50 percent in the slow technological progress case and fall by 33 percent in the rapid technological progress case (Figure 10).

Since the transmission and distribution margins to industrial gas customers are much lower than the margins for residential and commercial customers, responses to wide variations in the wellhead gas prices are more readily observed in industrial markets than in the residential and commercial markets where a larger proportion of the delivered price of gas is represented by nonfuel costs.

\section{Slow Technological Progress}

The slow technological progress case assumes that no equipment beyond the 1995 level of efficiency penetrates the industrial market over the forecast period. Although industry is faced with higher energy prices due to less efficient supply production technologies, the impact of less efficient manufacturing equipment requires industry to consume 6 percent more energy by the year 2015 than in the $A E O 96$ reference case. The energy-intensive industries
Figure 8. New Car Fuel Efficiency, 1995-2015

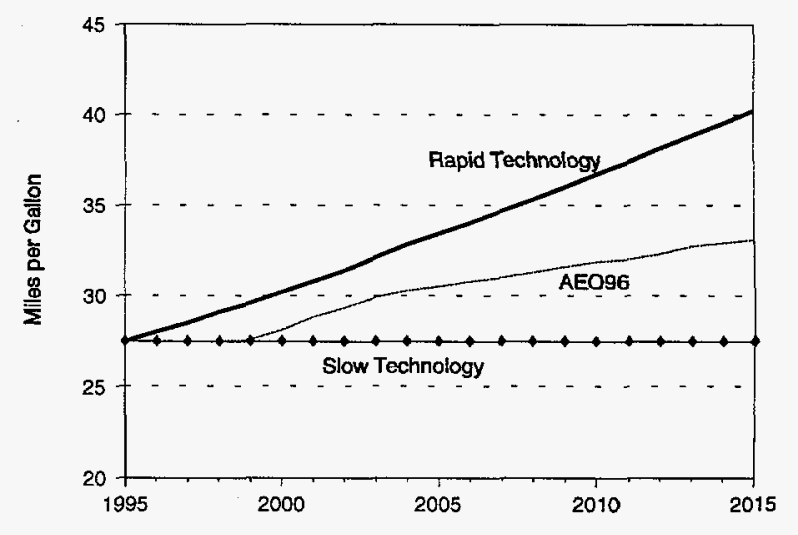

Source: Table B7.

consume 12 percent more energy than in the $A E O 96$ reference case, and the metal-based durables industries' consumption increases by 8 percent (Figure 11).

Higher energy expenditures are due to increased consumption from lower efficiencies and higher prices. While coal accounts for two-thirds of the consumption increase (1.3 quadrillion Btu), coal accounts for less than 10 percent of the increase in energy expenditures. In contrast, natural gas consumption declines slightly relative to the AEO96 reference case despite 50-percent higher natural gas prices.

Although the industrial sector consumption of natural gas in the slow technological progress case is only about 0.1 quadrillion Btu lower than the AEO96 reference case in absolute terms, delivered energy consumption by the industrial sector is about 7 percent higher in 2015 because of the lower end-use efficiencies. This implies that, in 2015, natural gas market share in the industrial sector is actually 3 percent lower than the AEO96 reference case. Since the price of natural gas to industrial markets is only 9 percent higher than the AEO96 reference case in 2005 and since industrial capital stock turns over very slowly, the 30percent price difference in 2010 and the 50- percent price difference in 2015 for delivered industrial natural gas prices have only a limited impact on natural gas consumption in the industrial sector. The 3-percent market share change is limited by capital stock turnover and the limited interfuel substitution potential in U.S. industrial processes.

Natural gas expenditures represent half of the total increase in energy expenditures in 2015 (Figure 12). Coal consumption in the slow technological progress case 
Figure 9. Industrial Energy Intensity, 1995-2015

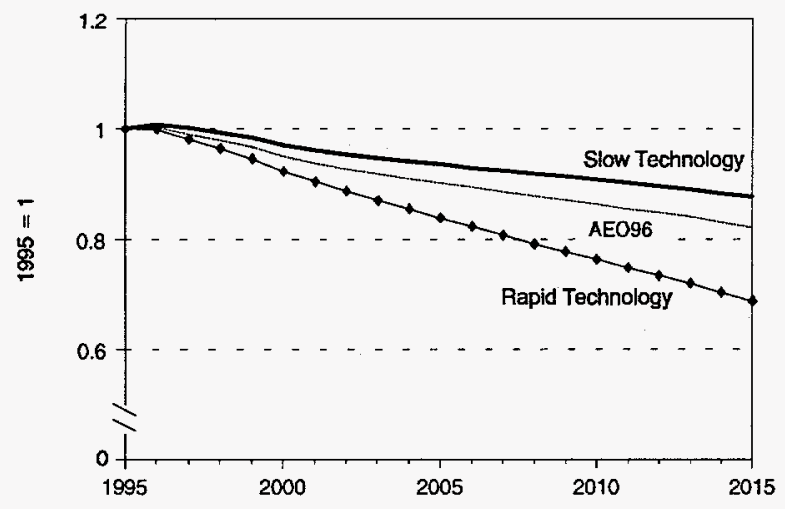

Source: AEO96 National Energy Modeling System runs TECH01.D031396A, AEO96B.D101995C, and TECH04.D031396B.

increases by 1.3 quadrillion Btu relative to the AEO96 reference case, and the delivered price of steam coal increases by $\$ 0.19$ per million Btu, from $\$ 1.31$ to $\$ 1.50$ per million Btu, resulting in increased expenditures of $\$ 2.32$ billion. Natural gas expenditures in the industrial sector are projected to increase from $\$ 38.0$ billion in the AEO96 reference case to $\$ 56.3$ billion in the slow technological progress case despite a net decrease of natural gas consumption of 0.1 quadrillion Btu. This reflects the influence of both the quantity dominance and the price changes for industrial natural gas on energy expenditures. The consumption increases coupled with the higher energy prices result in an increase in total energy expenditures of $\$ 38$ billion in 2015, a 24-percent increase over the AEO96 reference case. Cumulatively over the forecast, total expenditures in the slow technological progress case result in incremental energy expenditures in excess of $\$ 265$ billion, an almost 10-percent increase in cumulative expenditures over the $A E O 96$ reference case.

\section{Rapid Technological Progress}

The higher efficiency gains resulting from the rapid technology assumptions produce significant energy consumption and energy expenditure savings for the industrial market. By 2015, total purchased fuel consumption is projected to be 16 percent lower than in the $A E O 96$ reference case. Again, the energy-intensive industries respond to the case assumptions by consuming 19 percent less energy than in the $A E O 96$ reference case projections. Most of the energy savings result from the decrease in coal consumption, 33 percent lower than in the AEO96 reference case. As in the slow technological progress case, coal expenditures account for very little of the total change in energy expenditures. In this case, the natural gas price falls
Figure 10. Industrial Energy Price Changes Relative to AEO96, 2015

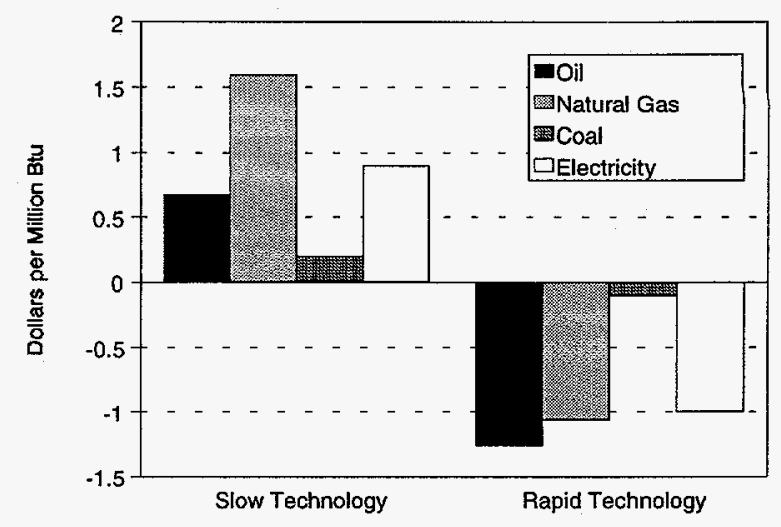

Source: Table B3.

by one-third while the petroleum price falls by one-fourth compared with the AEO96 reference case. As a result, oil accounts for most of the decline in energy expenditures followed by natural gas. These consumption patterns also impact total industrial energy expenditures. In 2015, the rapid technological progress assumptions imply a $\$ 52.5$ billion decrease in energy expenditures which is a 33-percent decrease relative to the $A E O 96$ reference case in 2015. In cumulative undiscounted expenditure terms, industry expends almost $\$ 450$ billion less by 2015 , which is a 15.7percent decrease relative to the $A E O 96$ reference case.

\section{Impacts of Technological Breakthroughs Across the Energy System}

This analysis establishes the importance of technological progress on supply prices, production, and end-use consumption. Assumed technological advances and rates of penetration on one side of the demand-supply equation reduce the potential for additional energy savings from the other side. That is, as advanced demand technologies succeed in displacing less efficient end-use technologies, the demand for each fuel form-electricity, natural gas, distillate fuel, and coal-tends to decline and diminishes the adoption of potentially successful supply technologies. For example, as electricity end-use technologies improve, the demand for electricity will decline, and consequently the market for new, more efficient supply technologies will be diminished. Alternatively, if supply technologies succeed, then the cost of supplying the fuel will decline, and the value of paying additional capital to buy higherefficiency end-use equipment will also be diminished. 
Figure 11. Consumption Changes by Industrial Subsector Relative to AEO96, 2015

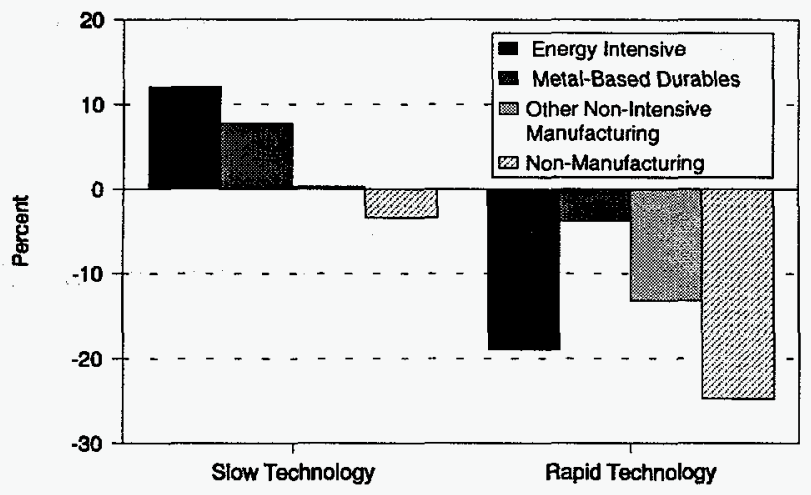

Source: AEO96 National Energy Modeling System runs TECH01.D031396A, AEO96B.D101995C, and TECH04.D031396B.

\section{Observations and Conclusions}

In this analysis, we began with relatively simple assumptions about what constitutes slow and rapid technological progress on the supply and demand for energy. The assumptions used for the AEO96 technology cases for each sector were used as the starting point for these integrated cases. These assumptions were modified slightly for the residential and commercial sectors to add price-responsive decisionmaking for the rapid technology case. Also, the industrial and the coal and oil and gas supply models do not represent individual technologies but use historically plausible technological progress rates to evaluate system impacts. In retrospect, the insights of this study were somewhat limited by the oversimplified approach used to define these two cases for each sector because technological change in the oil, gas, transportation, industrial, and coal markets was specified as autonomous change without explicit linkage to causal factors in energy markets. One of the major benefits of this study was to help us define technology cases so they can provide greater value to our clients. Some of the major insights of this study include:

- Successful R\&D in the oil and gas production and exploration industries will, all else being equal, reduce the cost of domestic crude oil and gas supply, increase the profitability of both domestic industries, increase oil and gas production, and reduce oil and gas prices in U.S. energy markets.

- Because crude oil prices are largely determined by an international market, advances in domestic oil exploration and production technologies reduce world oil prices to a lesser extent than comparable
Figure 12. Industrial Energy Expenditure Changes Relative to AEO96, 2015

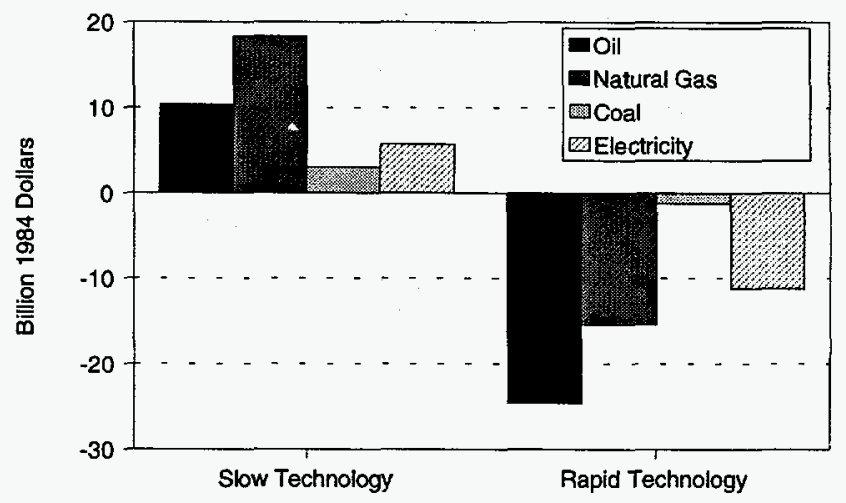

Source: AEO96 National Energy Modeling System runs TECH01.D031396A, AEO96B.D101995C, and TECH04.D031396B.

advances in domestic gas exploration and drilling technologies reduce U.S. natural gas wellhead prices.

- Under the cases considered, U.S. dependence on oil imports is minimized when rapid technology advances for both supply and demand are developed, commercialized, and adopted in the U.S. energy system.

- The rate of demand technological progress primarily affects consumption for fuels with secondary impacts on fuel prices; the rate of supply technological progress primarily affects fuel prices with some important secondary impacts on domestic production. Consequently, fuel consumption and carbon emissions are likely to be at their greatest with slower demand technology assumptions.

- Carbon emissions are at their lowest when demand technological progress is most rapid.

- Rapid deployment of higher efficiency equipment for demand tends to postpone the need for new generation and reduces the market potential for advanced generation technologies.

- Although none of the cases examined achieve carbon stabilization despite the favorable assumptions made in the rapid technology case, the results suggest that $R \& D$ investments that succeed in developing low-cost, high-efficiency end-use technologies and facilitate market acceptance of them could play a significant role in moderating carbon 
emissions in the United States. R\&D programs that improve the availability and market acceptance of cost-efficient transportation technologies coupled with successful oil and gas supply R\&D programs could have a significant impact on reducing imported oil dependence if these programs were successful.

- Relative to the assumptions of this study, technological progress for demand technologies has a greater potential impact in reducing oil imports and carbon emissions than progress for the supply technologies.
Numerous uncertainties cloud our ability to see with clarity the evolution of the U.S. energy system. The principal uncertainties relate to the menu of advanced technologies that might be commercially available in the future, their cost and performance characteristics, the rate at which new high-efficiency technologies are developed through public and private R\&D efforts, and how consumer choices will and can evolve over time. An empirical issue is the relationship between R\&D funding, technical success, and market acceptance. Public awareness and acceptance of superior technologies are likely to be key elements of the way choices are made in the future. 

Appendix A

Assumptions of the Technology Cases 



\section{Appendix A}

\section{Assumptions of the Technology Cases}

\section{Residential Energy Market}

The slow technological progress case assumes that all future equipment purchases are made based only on equipment available in 1995. This case further assumes that building shell efficiency will increase more slowly than in the reference case. In the reference case, the 2015 housing stock shell efficiency is 9 percent higher than in 1993 for heating and 6 percent for cooling. In the slow technological progress case, heating shell efficiency is assumed to increase only 3 percent, and cooling shell, 2 percent.

The rapid technology case assumes that all equipment purchases from 1995 forward are based on consumer discount rates of 7 percent-unlike the $A E O 96$ standalone case which assumed that the best available technology was always chosen irrespective of capital cost. In the rapid technology case, heating shell efficiency is assumed to increase by 17 percent and cooling shell efficiency by 12 percent.

\section{Commercial Energy Market}

The slow technological progress case assumes that all future equipment purchases are made based only on equipment available in 1995. This case further assumes that building shell efficiency will increase more slowly than in the AEO96 reference case. In the reference case, existing building shells are allowed to increase in efficiency by as much as 5 percent over 1992 levels. In the slow technology case, existing shell efficiency is assumed to be fixed at 1995 levels.

The rapid technology case assumes that all equipment purchases from 1995 forward are based on significantly lower discount rates than in the AEO96 reference case-unlike the $A E O 96$ standalone case which assumed that the best available technology was always chosen irrespective of capital cost. That is, in the rapid technological progress case, 50 percent of consumers are assumed to invest at the risk-free discount rate, 25 percent at the risk-free rate plus 10 percent, and 25 percent will invest at the risk-free rate plus 20 percent. In the rapid technology case, existing shell efficiency is assumed to increase to the levels of efficiency for new construction by 2015.

\section{Industrial Energy Market}

Over the 1970 to 1990 period, industrial energy intensity fell by 1.9 percent annually, due to energy conservation and the changing composition of industrial output. The rapid technology case approximates the 1.9-percent annual decline from 1995 through 2015 . This intensity decline is twice the rate of decline anticipated in the AEO96 reference case ( 0.9 percent). For this study, the composition of industrial output was held the same as in the $A E O 96$ reference case.

The slow technological progress case holds the energy efficiency of new plant and equipment constant over the forecast. New equipment and processes, however, typically are more energy efficient than those used in existing plants. As a result, the average energy intensity declines as old equipment is retired and new, more efficient, production capacity is added.

\section{Transportation Energy Market}

Over the 1970 to 1990 period, transportation energy efficiency rose by 1.9 percent annually for light-duty vehicles, 1.5 percent for freight trucks, 1.6 percent for rail locomotives and marine vessels, and 2.1 percent for aircraft. These rates were used to override the normal, fuel price dependent, endogenously-determined, efficiency improvement rates in the rapid technological progress case for the transportation sector.

The slow technological progress case assumes that new transportation vehicle fuel efficiencies are held constant at 1995 levels over the forecast.

\section{Electricity Generation Market}

The slow and rapid technology cases assume different capital costs for advanced generating technologies than in the AEO96 reference case. In the slow technology case, 
technological optimism and learning factors-which increase the nth-of-a-kind capital costs for new capacity-are doubled relative to the reference case and are applied to the first six units constructed. After six units are constructed, the cost increase due to technological optimism is removed and a learning factor of 14 percent-where capital costs are reduced by 14 percent for each doubling of capacity-is applied to the next 48 units constructed. After a total of 60 units have been built, capital costs are assumed to remain constant at the nth-ofa-kind costs for all subsequent construction. Conversely, the rapid technology case assumes a halving of the technological optimism factors relative to the reference case for the first two units and a learning factor of 6 percent applied to the next 18 units. After a total of 20 units have been built, capital costs are assumed to remain constant at the nth-of-a-kind costs for all subsequent construction (Table A1).

\section{Oil and Gas Supply}

Oil and gas technological progress rates for unproved resources and drilling costs were adjusted upward and downward by approximately 50 percent. This change affects both the size of reserve additions per new well and the number of new well completions. The specific variations in economically recoverable resources used in the analysis are shown in Table 33 and Table 34 of the

Table A1. Characteristics of New Generating Technologies

\begin{tabular}{|c|c|c|c|c|c|c|}
\hline Technology & $\begin{array}{c}\text { Year } \\
\text { Available }\end{array}$ & $\begin{array}{l}\text { Overnight } \\
\text { Capital Costs } \\
\text { (1987 dollars } \\
\text { per kilowatt) }\end{array}$ & $\begin{array}{l}1995 \text { of First- } \\
\text { of-a-Kind Heat } \\
\text { Rate (Btu per } \\
\text { kilowatthour) }\end{array}$ & $\begin{array}{c}2010 \text { Heat } \\
\text { Rate } \\
\text { (Btu per } \\
\text { kilowatthour) }\end{array}$ & $\begin{array}{l}\text { Fixed O\&M } \\
\text { (1987 } \\
\text { dollars per } \\
\text { kilowatt) }\end{array}$ & $\begin{array}{l}\text { Variable O\&M } \\
\text { (1987 dollars } \\
\text { per thousand } \\
\text { kilowatthours) }\end{array}$ \\
\hline Pulverized Coal . . . . . . . . & now & 1,190 & 9,961 & 8,142 & 40.5 & 1.9 \\
\hline Advanced Coal & 2000 & 1,008 & 8,730 & 7,582 & 39.4 & 1.0 \\
\hline Oil/Gas Steam . & now & 752 & 9,477 & 9,477 & 5.3 & 5.2 \\
\hline Advanced Combined-Cycle .. & 1999 & 332 & 7,300 & 5,687 & 21.0 & 0.4 \\
\hline Combustion Turbine ....... & now & 275 & 11,900 & 10,663 & 9.4 & 0.1 \\
\hline \multicolumn{7}{|l|}{ Advanced Combustion } \\
\hline Turbine. & 1999 & 600 & 9,000 & 7,935 & 26.7 & 0.5 \\
\hline Geothermal ....... & now & 2,093 & NA & NA & 69.0 & 0.0 \\
\hline Municipal Solid Waste & now & 4,389 & 16,000 & 16,000 & 12.8 & -3.8 \\
\hline Solar Thermal & 1999 & 1,045 & NA & NA & 19.8 & 0.0 \\
\hline Photovoltaic . . . . & 1999 & 1,705 & NA & NA & 5.1 & 0.0 \\
\hline Wind $\ldots \ldots \ldots \ldots \ldots$ & now & 740 & NA & NA & 20.9 & 0.0 \\
\hline
\end{tabular}

Btu $=$ British thermal units.

$O \& M=$ Operation and maintenance.

Notes: These costs characterize the technologies used in the AEO96 reference case. Overnight capital costs are for the nth of a kind unit, plus project contingencies. Since geothermal cost and performance parameters are specific for each of the 51 sites in the database, the value shown is an average for representative sites in California in 2015. Geothermal learning costs involve capital components for well, field, and two plant types, as well as operation and maintenance components. Details are provided in the model documentation. Because municipal solid waste (MSW) does not compete with other technologies in the model, these values are used only in calculating the average costs of electricity. Negative value for variable O\&M costs represents tipping fees for MSW disposal. Solar thermal only operates in Electricity Market Module regions 2,5 , and $10-13$ because of its requirement for significant direct, normal insolation.

Sources: Fossil and Nuclear: Office of Integrated Analysis and Forecasting. Electric Power Research Institute (EPRI), trade journals and other engineering estimates. Renewables: Values are derived by the Energy Information Administration, Office of Integrated Analysis and Forecasting in consultation with the organizations and publications as indicated for the following technologies. Solar Thermal: California Energy Commission Memorandum, "Technology Characterization for ER94," August 6, 1993 (Solar Central Receiver), Photovoltaic: Electric Power Research Institute - Technical Assessment Guide 1993 (EPRI-TAG 1993), (Fixed, flat plate, single tracking), Wind: EPRI-TAG 1993 (Horizontal axis), Geothermal: EPRI-TAG 1993 (Flash and Binary), Municipal Solid Waste: EPRI-TAG 1993, Biomass: EPRI-TAG 1993 (Advanced Gasifier-Combined Cycle). 
AEO96 assumptions, available on the EIA Home Page at ftp:/ / ftp.eia.doe.gov/pub/forecasting/aeo96/assumption/aeo96asu.pdf.

\section{Coal Supply}

In the reference case, labor productivity is assumed to increase at an average rate of 3.6 percent per year through 2015. The technology cases increased and decreased the annual growth rates by 50 percent in each year after 1995 . For example, a 4-percent productivity rate specific to a given year, mine type, and region in the reference case was set to 6 percent in the high productivity case and to 2 percent in the low productivity case. This resulted in average productivity growth rates of 5.1 percent per year in the high productivity case and 2.2 percent per year in the low productivity case. 

Appendix B

Comparison Tables Illustrating the Slow Technological Progress Case, the AEO96 Reference Case, and the Rapid Technological Progress Case 


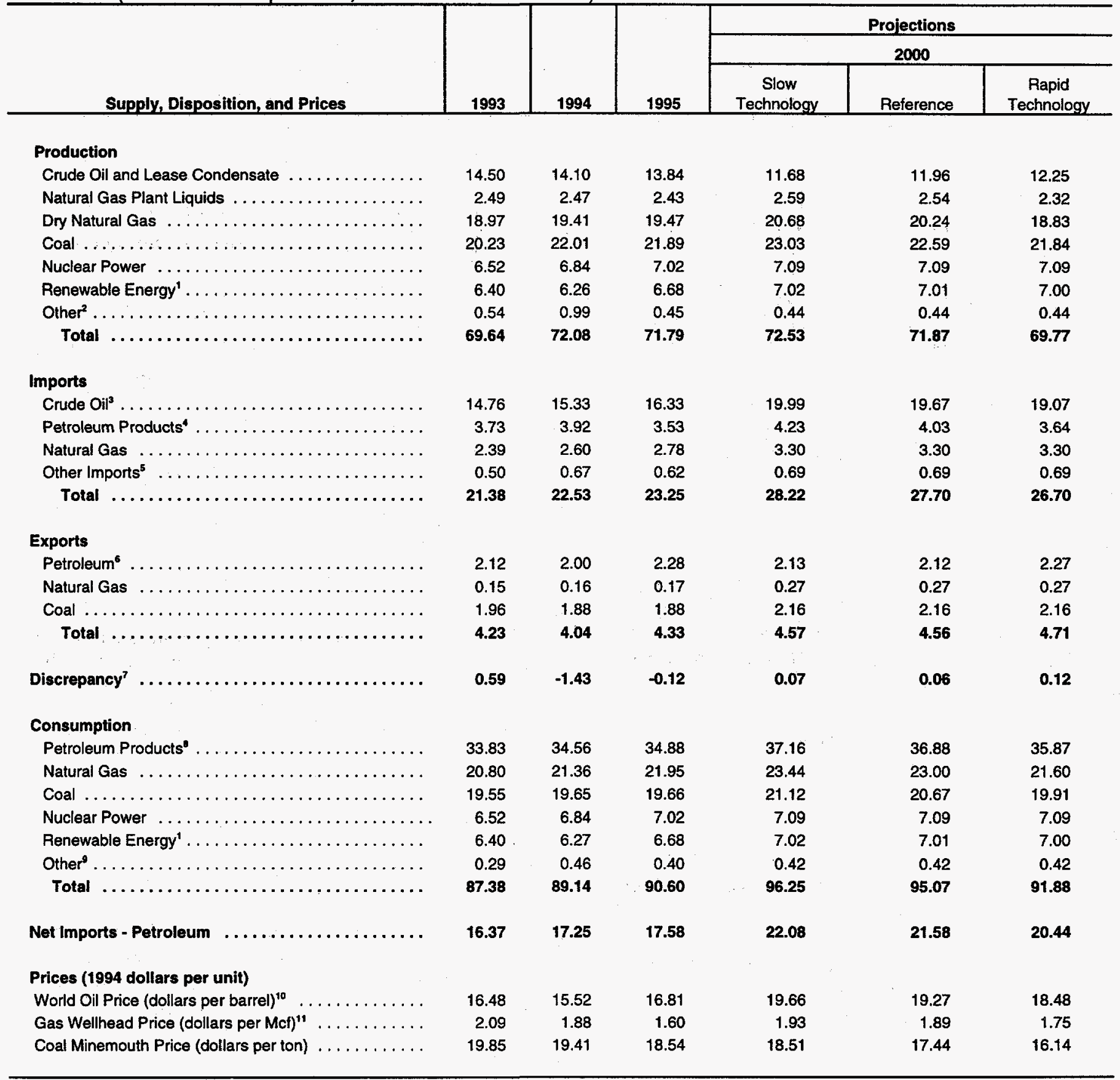

Includes utility and nonutility electricity from hydroelectric, wood and wood waste, municipal solid waste, other biomass, wind, photovoltaic and solar thermal sources; non-electric

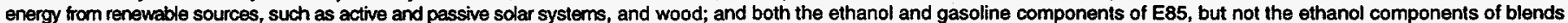
less than 85 percent. Excludes nonmarketed renewable energy. See Table 18 for selected nonmarketed residential and commercial renewable energy.

Includes liquid hydrogen, methanol, supplemental natural gas, and some domestic inputs to refineries.

Includes imports of crude oil for the Strategic Petroleum Reserve.

"Includes imports of finished petroleum products, imports of unfinished oils, alcohols, ethers, and blending components.

5 Includes coal, coal coke (net), and electricity (net).

Includes crude oil and petroleum products.

'Balancing item. Includes unaccounted for supply, losses, gains, and net storage withdraws.

"Includes natural gas plant liquids, crude oil consumed as a fuel, and nonpetroleum based liquids for blending, such as ethanol.

includes net electricity imports, methanol, and liquid hydrogen.

${ }^{10}$ Average refiner acquisition cost for imported crude oil.

"Represents lower 48 onshore and offshore supplies. 
Table B1. Total Energy Supply and Disposition Summary (Continued) (Quadrillion Btu per Year, Unless Otherwise Noted)

\begin{tabular}{|c|c|c|c|c|c|c|c|c|c|c|c|}
\hline \multicolumn{9}{|c|}{ Projections } & \multirow{2}{*}{\multicolumn{3}{|c|}{$\begin{array}{c}\text { Annual Growth } \\
\text { 1994-2015 (percent) }\end{array}$}} \\
\hline \multicolumn{3}{|c|}{2005} & \multicolumn{3}{|c|}{2010} & \multicolumn{3}{|c|}{2015} & & & \\
\hline $\begin{array}{c}\text { Slow } \\
\text { Technology }\end{array}$ & Reference & $\begin{array}{c}\text { Rapid } \\
\text { Technology }\end{array}$ & $\begin{array}{c}\text { Slow } \\
\text { Technology }\end{array}$ & Reference & $\begin{array}{c}\text { Rapid } \\
\text { Technology }\end{array}$ & $\begin{array}{c}\text { Slow } \\
\text { Technology }\end{array}$ & Reference & $\begin{array}{c}\text { Rapid } \\
\text { Technology }\end{array}$ & $\begin{array}{c}\text { Slow } \\
\text { Technology }\end{array}$ & Reference & $\begin{array}{c}\text { Rapid } \\
\text { Technology }\end{array}$ \\
\hline 10.63 & 11.10 & 11.61 & 10.87 & 11.51 & 11.84 & 11.40 & 12.30 & 12.79 & -1.0 & -0.6 & -0.5 \\
\hline 2.84 & 2.74 & 2.44 & 3.08 & 2.97 & 2.56 & 3.19 & 3.20 & 2.84 & 1.2 & 1.2 & 0.7 \\
\hline 22.55 & 21.89 & 19.32 & 24.41 & 23.52 & 20.48 & 25.58 & 25.72 & 23.03 & 1.3 & 1.3 & 0.8 \\
\hline 24.85 & 23.95 & 22.57 & 26.41 & 24.94 & 23.29 & 28.90 & 26.14 & 24.17 & 1.3 & 0.8 & 0.4 \\
\hline 6.93 & 6.93 & 6.93 & 6.52 & 6.52 & 6.52 & 4.63 & 4.63 & 4.63 & -1.8 & -1.8 & -1.8 \\
\hline 7.46 & 7.38 & 7.28 & 7.85 & 7.78 & 7.57 & 8.53 & 8.51 & 7.97 & 1.5 & 1.5 & 1.2 \\
\hline 0.51 & 0.50 & 0.49 & 0.56 & 0.55 & 0.53 & 0.62 & 0.58 & 0.55 & -2.2 & -2.5 & -2.7 \\
\hline 75.77 & 74.50 & 70.64 & 79.72 & 77.79 & 72.78 & 82.85 & 81.08 & 75.99 & 0.7 & 0.6 & 0.3 \\
\hline 22.30 & 21.13 & 19.43 & 23.23 & 21.05 & 19.11 & 23.05 & 20.87 & 18.21 & 2.0 & 1.5 & 0.8 \\
\hline 5.46 & 5.23 & 4.27 & 6.36 & 6.16 & 4.10 & 8.12 & 6.40 & 3.54 & 3.5 & 2.4 & -0.5 \\
\hline 3.48 & 3.48 & 3.48 & 3.84 & 3.84 & 3.36 & 4.42 & 4.42 & 2.95 & 2.5 & 2.5 & 0.6 \\
\hline 0.59 & 0.59 & 0.57 & 0.61 & 0.61 & 0.59 & 0.56 & 0.56 & 0.54 & -0.9 & -0.9 & -1.0 \\
\hline 31.83 & 30.43 & 27.76 & 34.04 & 31.65 & 27.17 & 36.15 & 32.25 & 25.24 & 2.3 & 1.7 & 0.5 \\
\hline 1.90 & 1.88 & 1.92 & 1.84 & 1.77 & 2.01 & 1.89 & 1.91 & 2.39 & -0.3 & -0.2 & 0.9 \\
\hline 0.31 & 0.31 & 0.31 & 0.32 & 0.32 & 0.32 & 0.33 & 0.33 & 0.33 & 3.5 & 3.5 & 3.5 \\
\hline 2.37 & 2.37 & 2.37 & 2.66 & 2.66 & 2.67 & 3.14 & 3.14 & 3.15 & 2.5 & 2.5 & 2.5 \\
\hline 4.58 & 4.56 & 4.60 & 4.82 & 4.74 & 4.99 & 5.36 & 5.38 & 5.86 & 1.4 & 1.4 & 1.8 \\
\hline 0.01 & 0.01 & 0.05 & -0.08 & -0.01 & -0.01 & 0.07 & 0.07 & 0.09 & N/A & NA & N/A \\
\hline 40.08 & 39.12 & 36.62 & 42.39 & 40.68 & 36.36 & 44.69 & 41.69 & 35.86 & 1.2 & 0.9 & 0.2 \\
\hline 25.45 & 24.79 & 22.24 & 27.65 & 26.76 & 23.26 & 29.42 & 29.52 & 25.37 & 1.5 & 1.6 & 0.8 \\
\hline 22.79 & 21.83 & 20.45 & 24.03 & 22.55 & 20.83 & 26.03 & 23.27 & 21.24 & 1.3 & 0.8 & 0.4 \\
\hline 6.93 & 6.93 & 6.93 & 6.52 & 6.52 & 6.52 & 4.63 & 4.63 & 4.63 & -1.8 & -1.8 & -1.8 \\
\hline 7.46 & 7.38 & 7.28 & 7.86 & 7.78 & 7.58 & 8.54 & 8.52 & 7.98 & 1.5 & 1.5 & 1.2 \\
\hline 0.33 & 0.33 & 0.33 & 0.41 & 0.40 & 0.40 & 0.40 & 0.40 & 0.39 & -0.6 & -0.7 & -0.9 \\
\hline 103.04 & 100.38 & 93.85 & 108.86 & 104.69 & 94.95 & 113.72 & 108.02 & 95.46 & 1.2 & 0.9 & 0.3 \\
\hline 25.86 & 24.48 & 21.78 & 27.75 & 25.44 & 21.20 & 29.28 & 25.36 & 19.36 & 2.6 & 1.9 & 0.6 \\
\hline 22.99 & 21.86 & 19.77 & 25.59 & 23.70 & 20.55 & 28.58 & 25.43 & 21.17 & 3.0 & 2.4 & 1.5 \\
\hline 2.23 & 1.99 & 1.52 & 2.99 & 2.15 & 1.38 & 4.18 & 2.57 & 1.55 & 3.9 & 1.5 & -0.9 \\
\hline 19.95 & 17.68 & 15.74 & 20.22 & 17.43 & 15.59 & 20.95 & 17.39 & 15.26 & 0.4 & -0.5 & -1.1 \\
\hline
\end{tabular}

Btu $=$ British thermal unit.

Mct = Thousand cubic feet.

N/A = Not applicable.

Note: Totals may not equal sum of components due to independent rounding. Figures for 1993 and 1994 may differ from published data due to internal conversion factors.

Sources: 1993 natural gas values: Energy Information Administration (EIA), Natural Gas Annual 1993, DOE/EIA-0131(93) (Washington, DC, October 1994). 1993 coal minemouth prices: ElA, Coal Industry Amual 1993, DOE/EIA-0584(93) (Washington, DC, December 1994). Other 1993 values: EIA, Annual Energy Review 1994, DOE/ElA-0384(94) (Washington,

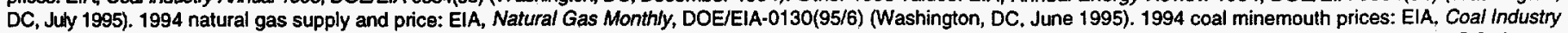
Annual 1994, DOE/EIA-0584(94) (Washington, DC, December 1995), 1994 coal production and exports: ElA, Monthly Energy Review, DOE/EIA-0035(95/08) (Washington, DC, August

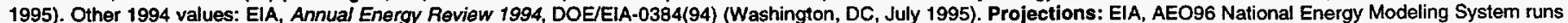
TECH01.D031396A (Slow Technology), AEO96B.D101995C (reference), and TECH04.D031396B (Rapid Technology). 
Table B2. Energy Consumption by Sector and Source

(Quadrillion Btu per Year, Unless Otherwise Noted)

\begin{tabular}{|c|c|c|c|c|c|c|}
\hline \multirow[b]{3}{*}{ Sector and Source } & \multirow[b]{3}{*}{1993} & \multirow[b]{3}{*}{1994} & \multirow[b]{3}{*}{1995} & \multicolumn{3}{|c|}{ Projections } \\
\hline & & & & \multicolumn{3}{|c|}{2000} \\
\hline & & & & $\begin{array}{c}\text { Slow } \\
\text { Technology }\end{array}$ & Reference & $\begin{array}{c}\text { Rapid } \\
\text { Technology }\end{array}$ \\
\hline
\end{tabular}

\section{Energy Consumption}

\begin{tabular}{|c|c|c|c|c|c|c|}
\hline \multicolumn{7}{|l|}{ Residential } \\
\hline Distillate Fuel & 0.91 & 0.87 & 0.84 & 0.86 & 0.84 & 0.83 \\
\hline Kerosene $\ldots \ldots \ldots \ldots \ldots \ldots \ldots \ldots \ldots$ & 0.08 & 0.07 & 0.07 & 0.07 & 0.07 & 0.07 \\
\hline Liquefied Petroleum Gas . . . . . . . . . . . . . . & 0.40 & 0.41 & 0.40 & 0.39 & 0.39 & 0.38 \\
\hline Petroleum Subtotal . . . . . . . . . . . . . . & 1.39 & 1.35 & 1.31 & 1.32 & 1.30 & 1.28 \\
\hline Natural Gas $\ldots \ldots \ldots \ldots \ldots \ldots \ldots \ldots$ & 5.10 & 5.03 & 5.00 & 5.28 & 5.22 & 5.10 \\
\hline 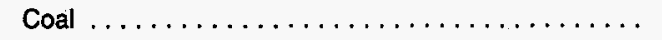 & 0.06 & 0.06 & 0.05 & 0.05 & 0.05 & 0.05 \\
\hline Renewable Energy ${ }^{\top} \ldots \ldots \ldots \ldots \ldots \ldots \ldots$ & 0.57 & 0.55 & 0.55 & 0.58 & 0.57 & 0.56 \\
\hline Electricity $\ldots \ldots \ldots \ldots \ldots \ldots \ldots \ldots \ldots \ldots \ldots \ldots \ldots \ldots \ldots$ & 3.39 & 3.43 & 3.49 & 3.72 & 3.70 & 3.57 \\
\hline Delivered Energy $\ldots \ldots \ldots \ldots \ldots \ldots \ldots$ & 10.51 & 10.41 & 10.40 & 10.96 & 10.84 & 10.55 \\
\hline Electricity Related Losses . . . . . . . . . . . . . . & 7.57 & 7.63 & 7.73 & 8.02 & 7.96 & 7.64 \\
\hline Total $\ldots \ldots \ldots \ldots \ldots \ldots \ldots \ldots \ldots \ldots \ldots \ldots \ldots \ldots \ldots$ & 18.08 & 18.04 & 18.13 & 18.98 & 18.80 & 18.19 \\
\hline \multicolumn{7}{|l|}{ Commercial } \\
\hline Distillate Fuel $\ldots \ldots \ldots \ldots \ldots \ldots \ldots \ldots$ & 0.46 & 0.42 & 0.42 & 0.42 & 0.42 & 0.45 \\
\hline Residual Fuel $\ldots \ldots \ldots \ldots \ldots \ldots \ldots \ldots \ldots$ & 0.17 & 0.18 & 0.18 & 0.18 & 0.18 & 0.18 \\
\hline Kerosene $\ldots \ldots \ldots \ldots \ldots \ldots \ldots \ldots \ldots$ & 0.01 & 0.01 & 0.01 & 0.01 & 0.01 & 0.01 \\
\hline Liquefied Petroleum Gas . . . . . . . . . . . . . & 0.07 & 0.05 & 0.05 & 0.05 & 0.05 & 0.05 \\
\hline Motor Gasoline $^{2} \ldots \ldots \ldots \ldots \ldots \ldots$ & 0.03 & 0.07 & 0.07 & 0.07 & 0.07 & 0.07 \\
\hline Petroleum Subtotal $\ldots \ldots \ldots \ldots \ldots \ldots$ & 0.75 & 0.73 & 0.73 & 0.74 & 0.74 & 0.77 \\
\hline Natural Gas $\ldots \ldots \ldots \ldots \ldots \ldots \ldots \ldots \ldots$ & 2.98 & 3.01 & 3.04 & 3.21 & 3.20 & 3.09 \\
\hline Coal $\ldots \ldots \ldots \ldots \ldots \ldots \ldots \ldots \ldots \ldots \ldots \ldots$ & 0.08 & 0.08 & 0.08 & 0.09 & 0.09 & 0.09 \\
\hline Renewable Energy ${ }^{3} \ldots \ldots \ldots \ldots \ldots \ldots$ & 0.00 & 0.00 & 0.00 & 0.00 & 0.00 & 0.00 \\
\hline Electricity $\ldots \ldots \ldots \ldots \ldots \ldots \ldots \ldots \ldots$ & 3.01 & 3.13 & 3.20 & 3.37 & 3.36 & 3.10 \\
\hline Delivered Energy $\ldots \ldots \ldots \ldots \ldots \ldots \ldots$ & 6.83 & 6.96 & 7.06 & 7.41 & 7.39 & 7.05 \\
\hline Electricity Related Losses . . . . . . . . . . . . & 6.72 & 6.95 & 7.08 & 7.27 & 7.23 & 6.65 \\
\hline Total $\ldots \ldots \ldots \ldots \ldots \ldots \ldots \ldots \ldots \ldots \ldots \ldots \ldots$ & 13.55 & 13.91 & 14.14 & 14.68 & 14.62 & 13.70 \\
\hline \multicolumn{7}{|l|}{ Industrial ${ }^{4}$} \\
\hline$\ldots \ldots \ldots \ldots \ldots \ldots \ldots \ldots \ldots$ & 1.10 & 1.15 & 1.16 & 1.22 & 1.21 & 1.17 \\
\hline Liquefied Petroleum Gas . . . . . . . . . . . . & 1.79 & 1.87 & 1.92 & 1.97 & 1.97 & 1.91 \\
\hline Petrochemical Feedstocks $\ldots \ldots \ldots \ldots \ldots \ldots$ & 1.19 & 1.25 & 1.25 & 1.27 & 1.27 & 1.24 \\
\hline Residual Fuel $\ldots \ldots \ldots \ldots \ldots \ldots \ldots \ldots$ & 0.45 & 0.47 & 0.47 & 0.51 & 0.48 & 0.45 \\
\hline Motor Gasoline $^{2} \ldots \ldots \ldots \ldots \ldots \ldots \ldots$ & 0.18 & 0.19 & 0.19 & 0.20 & 0.20 & 0.19 \\
\hline Other Petroleum ${ }^{5} \ldots \ldots \ldots \ldots \ldots \ldots \ldots$ & 3.69 & 3.90 & 3.92 & 4.09 & 4.07 & 4.01 \\
\hline Petroleum Subtotal $\ldots \ldots \ldots \ldots \ldots \ldots$ & 8.41 & 8.83 & 8.92 & 9.26 & 9.21 & 8.98 \\
\hline Natural Gas ${ }^{6} \ldots \ldots \ldots \ldots \ldots \ldots \ldots \ldots \ldots$ & 9.07 & 9.34 & 9.76 & 10.74 & 10.49 & 10.26 \\
\hline 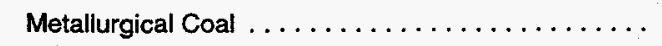 & 0.84 & 0.84 & 0.81 & 0.88 & 0.71 & 0.68 \\
\hline Steam Coal $\ldots \ldots \ldots \ldots \ldots \ldots \ldots \ldots$ & 1.66 & 1.69 & 1.67 & 1.98 & 1.73 & 1.56 \\
\hline Net Coal Coke Imports $\ldots \ldots \ldots \ldots \ldots \ldots$ & 0.02 & 0.02 & 0.02 & 0.03 & 0.03 & 0.02 \\
\hline Coal Subtotal $\ldots \ldots \ldots \ldots \ldots \ldots \ldots \ldots$ & 2.52 & 2.55 & 2.51 & 2.88 & 2.47 & 2.26 \\
\hline Renewable Energy ${ }^{7} \ldots \ldots \ldots \ldots \ldots \ldots$ & 2.08 & 2.16 & 2.19 & 2.36 & 2.36 & 2.36 \\
\hline Electricity $\ldots \ldots \ldots \ldots \ldots \ldots \ldots \ldots \ldots \ldots$ & 3.33 & 3.39 & 3.43 & 3.62 & 3.61 & 3.51 \\
\hline Delivered Energy $\ldots \ldots \ldots \ldots \ldots \ldots \ldots$ & 25.42 & 26.27 & 26.80 & 28.87 & 28.15 & 27.38 \\
\hline Electricity Related Losses . . . . . . . . . . . . . & 7.43 & 7.53 & 7.58 & 7.80 & 7.78 & 7.53 \\
\hline Total $\ldots \ldots \ldots \ldots \ldots \ldots \ldots \ldots \ldots \ldots$ & 32.85 & 33.80 & 34.38 & 36.67 & 35.93 & 34.91 \\
\hline
\end{tabular}


Table B2. Energy Consumption by Sector and Source (Continued) (Quadrillion Btu per Year, Unless Otherwise Noted)

\begin{tabular}{|c|c|c|c|c|c|c|c|c|c|c|c|}
\hline \multicolumn{9}{|c|}{ Projections } & \multirow{2}{*}{\multicolumn{3}{|c|}{$\begin{array}{c}\text { Annual Growth } \\
\text { 1994-2015 (percent) }\end{array}$}} \\
\hline \multicolumn{3}{|c|}{2005} & \multicolumn{3}{|c|}{2010} & \multicolumn{3}{|c|}{2015} & & & \\
\hline $\begin{array}{c}\text { Slow } \\
\text { Technology }\end{array}$ & Reference & $\begin{array}{c}\text { Rapid } \\
\text { Technology }\end{array}$ & $\begin{array}{c}\text { Slow } \\
\text { Technology }\end{array}$ & Reference & $\begin{array}{c}\text { Rapid } \\
\text { Technology }\end{array}$ & $\begin{array}{c}\text { Slow } \\
\text { Technology }\end{array}$ & Reference & $\begin{array}{c}\text { Rapid } \\
\text { Technology }\end{array}$ & $\begin{array}{c}\text { Slow } \\
\text { Technology }\end{array}$ & Reference & $\begin{array}{c}\text { Rapid } \\
\text { Technology }\end{array}$ \\
\hline
\end{tabular}

\begin{tabular}{|c|c|c|c|c|c|c|c|c|c|c|c|}
\hline 0.85 & 0.82 & 0.80 & 0.84 & 0.80 & 0.77 & 0.86 & 0.80 & 0.74 & -0.1 & -0.4 & -0.7 \\
\hline 0.07 & 0.06 & 0.06 & 0.06 & 0.06 & 0.06 & 0.06 & 0.06 & 0.06 & -0.5 & -0.6 & -0.7 \\
\hline 0.37 & 0.36 & 0.36 & 0.35 & 0.34 & 0.33 & 0.35 & 0.33 & 0.32 & -0.8 & -1.0 & -1.1 \\
\hline 1.28 & 1.24 & 1.22 & 1.26 & 1.20 & 1.16 & 1.27 & 1.19 & 1.12 & -0.3 & -0.6 & -0.9 \\
\hline 0.05 & 0.05 & 0.04 & 0.05 & 0.05 & 0.04 & 0.05 & 0.04 & 0.03 & -0.4 & -1.2 & -2.2 \\
\hline 0.60 & 0.58 & 0.55 & 0.62 & 0.59 & 0.54 & 0.64 & 0.59 & 0.53 & 0.7 & 0.3 & -0.2 \\
\hline 4.07 & 4.00 & 3.76 & 4.53 & 4.37 & 4.02 & 5.09 & 4.84 & 4.39 & 1.9 & 1.7 & 1.2 \\
\hline 11.47 & 11.20 & 10.69 & 12.15 & 11.71 & 10.92 & 12.93 & 12.28 & 11.28 & 1.0 & 0.8 & 0.4 \\
\hline 8.68 & 8.52 & 7.94 & 9.37 & 9.07 & 8.39 & 9.87 & 9.40 & 8.88 & 1.2 & 1.0 & 0.7 \\
\hline 20.15 & 19.73 & 18.63 & 21.52 & 20.78 & 19.31 & 22.80 & 21.68 & 20.16 & 1.1 & 0.9 & 0.5 \\
\hline 0.41 & 0.41 & 0.45 & 0.40 & 0.40 & 0.44 & 0.40 & 0.39 & 0.42 & -0.2 & -0.4 & 0.0 \\
\hline 0.18 & 0.18 & 0.18 & 0.19 & 0.19 & 0.19 & 0.19 & 0.19 & 0.19 & 0.3 & 0.3 & 0.3 \\
\hline 0.01 & 0.01 & 0.01 & 0.01 & 0.01 & 0.01 & 0.01 & 0.01 & 0.01 & 0.7 & 0.7 & 0.7 \\
\hline 0.06 & 0.06 & 0.06 & 0.06 & 0.06 & 0.06 & 0.06 & 0.06 & 0.06 & 1.0 & 1.0 & 1.0 \\
\hline 0.74 & 0.74 & 0.78 & 0.73 & 0.73 & 0.77 & 0.74 & 0.72 & 0.75 & 0.0 & -0.1 & 0.1 \\
\hline 3.31 & 3.31 & 3.16 & 3.40 & 3.42 & 3.25 & 3.44 & 3.51 & 3.35 & 0.6 & 0.7 & 0.5 \\
\hline 0.09 & 0.09 & 0.09 & 0.09 & 0.09 & 0.09 & 0.10 & 0.10 & 0.10 & 0.9 & 0.9 & 0.9 \\
\hline 0.00 & 0.00 & 0.00 & 0.00 & 0.00 & 0.00 & 0.00 & 0.00 & 0.00 & 0.7 & 0.7 & 0.7 \\
\hline 3.58 & 3.55 & 3.15 & 3.83 & 3.77 & 3.26 & 4.14 & 4.06 & 3.42 & 1.3 & 1.2 & 0.4 \\
\hline 7.73 & 7.68 & 7.18 & 8.05 & 8.02 & 7.37 & 8.41 & 8.40 & 7.63 & 0.9 & 0.9 & 0.4 \\
\hline 7.65 & 7.55 & 6.66 & 7.93 & 7.82 & 6.82 & 8.02 & 7.88 & 6.93 & 0.7 & 0.6 & 0.0 \\
\hline 15.38 & 15.24 & 13.84 & 15.98 & 15.84 & 14.19 & 16.42 & 16.28 & 14.56 & 0.8 & 0.8 & 0.2 \\
\hline 1.29 & 1.28 & 1.15 & 1.35 & 1.34 & 1.06 & 1.59 & 1.40 & 0.96 & 1.6 & 0.9 & -0.9 \\
\hline 2.10 & 2.11 & 1.89 & 2.19 & 2.19 & 1.75 & 2.29 & 2.30 & 1.62 & 1.0 & 1.0 & -0.7 \\
\hline 1.36 & 1.36 & 1.22 & 1.42 & 1.42 & 1.12 & 1.48 & 1.48 & 0.98 & 0.8 & 0.8 & -1.2 \\
\hline 0.54 & 0.49 & 0.44 & 0.57 & 0.49 & 0.36 & 0.60 & 0.52 & 0.40 & 1.1 & 0.4 & -0.8 \\
\hline 0.21 & 0.21 & 0.19 & 0.22 & 0.22 & 0.18 & 0.23 & 0.23 & 0.16 & 1.0 & 1.0 & -0.9 \\
\hline 4.25 & 4.16 & 3.88 & 4.41 & 4.22 & 3.67 & 4.59 & 4.32 & 3.46 & 0.8 & 0.5 & -0.6 \\
\hline 0.84 & 0.62 & 0.56 & 0.81 & 0.55 & 0.47 & 0.79 & 0.48 & 0.39 & -0.3 & -2.6 & -3.6 \\
\hline 2.27 & 1.76 & 1.39 & 2.71 & 1.83 & 1.29 & 3.26 & 1.96 & 1.25 & 3.2 & 0.7 & -1.4 \\
\hline 0.04 & 0.04 & 0.02 & 0.04 & 0.04 & 0.02 & 0.04 & 0.04 & 0.02 & 2.5 & 2.5 & -1.4 \\
\hline 3.15 & 2.42 & 1.97 & 3.56 & 2.42 & 1.77 & 4.09 & 2.48 & 1.65 & 2.3 & -0.1 & -2.0 \\
\hline 2.56 & 2.56 & 2.57 & 2.70 & 2.71 & 2.71 & 2.85 & 2.86 & 2.86 & 1.3 & 1.3 & 1.3 \\
\hline 3.91 & 3.86 & 3.63 & 4.14 & 4.04 & 3.67 & 4.36 & 4.23 & 3.69 & 1.2 & 1.1 & 0.4 \\
\hline 30.83 & 29.58 & 27.49 & 32.35 & 30.60 & 26.99 & 33.84 & 31.68 & 26.30 & 1.2 & 0.9 & 0.0 \\
\hline 8.35 & 8.22 & 7.68 & 8.56 & 8.38 & 7.67 & 8.46 & 8.22 & 7.47 & 0.6 & 0.4 & 0.0 \\
\hline 39.18 & 37.81 & 35.16 & 40.92 & 38.98 & 34.66 & $\mathbf{4 2 . 3 0}$ & 39.89 & 33.77 & 1.1 & 0.8 & 0.0 \\
\hline
\end{tabular}


Table B2. Energy Consumption by Sector and Source (Continued) (Quadrillion Btu per Year, Unless Otherwise Noted)

\begin{tabular}{|c|c|c|c|c|c|c|}
\hline \multirow[b]{3}{*}{ Sector and Source } & \multirow[b]{3}{*}{1993} & \multirow[b]{3}{*}{1994} & \multirow[b]{3}{*}{1995} & \multicolumn{3}{|c|}{ Projections } \\
\hline & & & & \multicolumn{3}{|c|}{2000} \\
\hline & & & & $\begin{array}{c}\text { Slow } \\
\text { Technology }\end{array}$ & Reference & $\begin{array}{c}\text { Rapid } \\
\text { Technology }\end{array}$ \\
\hline \multicolumn{7}{|l|}{ Transportation } \\
\hline Distillate Fuel $\ldots \ldots \ldots \ldots \ldots \ldots \ldots \ldots \ldots$ & 3.91 & 4.17 & 4.21 & 4.64 & 4.58 & 4.34 \\
\hline Jet Fuel $^{8} \ldots \ldots \ldots \ldots \ldots \ldots \ldots \ldots \ldots$ & 3.03 & 3.15 & 3.27 & 3.73 & 3.72 & 3.70 \\
\hline$\ldots \ldots \ldots \ldots \ldots \ldots \ldots \ldots \ldots$ & 14.13 & 14.21 & 14.54 & 15.45 & 15.35 & 15.00 \\
\hline Residual Fuel $\ldots \ldots \ldots \ldots \ldots \ldots \ldots \ldots \ldots$ & 0.91 & 0.90 & 0.94 & 1.07 & 1.07 & 1.06 \\
\hline Liquefied Petroleum Gas . . . . . . . . . . . . . . . . . & 0.02 & 0.03 & 0.02 & 0.02 & 0.02 & 0.02 \\
\hline Other Petroleum $^{9} \ldots \ldots \ldots \ldots \ldots \ldots \ldots \ldots$ & 0.20 & 0.20 & 0.21 & 0.22 & 0.22 & 0.22 \\
\hline$\ldots \ldots \ldots \ldots \ldots \ldots \ldots \ldots \ldots$ & 22.20 & 22.66 & 23.19 & 25.12 & 24.94 & 24.33 \\
\hline Pipeline Fuel Natural Gas $\ldots \ldots \ldots \ldots \ldots \ldots \ldots$ & 0.64 & 0.66 & 0.68 & 0.68 & 0.67 & 0.64 \\
\hline Compressed Natural Gas $\ldots \ldots \ldots \ldots \ldots \ldots \ldots \ldots$ & 0.01 & 0.01 & 0.01 & 0.01 & 0.01 & 0.01 \\
\hline Renewable Energy $(E 85)^{10} \ldots \ldots \ldots \ldots \ldots \ldots \ldots$ & 0.00 & 0.00 & 0.00 & 0.00 & 0.00 & 0.00 \\
\hline$\ldots \ldots \ldots \ldots \ldots \ldots \ldots \ldots \ldots \ldots$ & 0.00 & 0.00 & 0.00 & 0.00 & 0.00 & 0.00 \\
\hline Liquid Hydrogen $\ldots \ldots \ldots \ldots \ldots \ldots \ldots$ & 0.00 & 0.00 & 0.00 & 0.00 & 0.00 & 0.00 \\
\hline 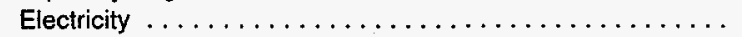 & 0.02 & 0.02 & 0.02 & 0.03 & 0.03 & 0.03 \\
\hline Delivered Energy $\ldots \ldots \ldots \ldots \ldots \ldots \ldots \ldots \ldots \ldots \ldots$ & 22.87 & 23.34 & 23.90 & 25.84 & 25.66 & 25.02 \\
\hline Electricity Related Losses . . . . . . . . . . . . . . . . & 0.04 & 0.05 & 0.05 & 0.07 & 0.07 & 0.06 \\
\hline 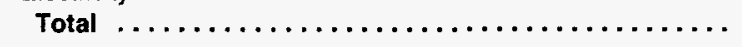 & 22.91 & 23.39 & 23.94 & 25.91 & 25.72 & 25.08 \\
\hline \multicolumn{7}{|l|}{ Delivered Energy Consumption for All Sectors } \\
\hline 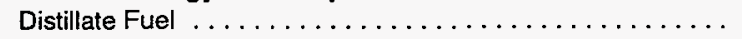 & 6.39 & 6.61 & 6.63 & 7.14 & 7.05 & 6.80 \\
\hline 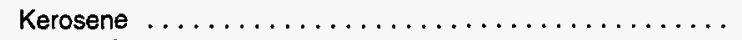 & 0.10 & 0.09 & 0.09 & 0.09 & 0.09 & 0.09 \\
\hline let Fuel $^{8} \ldots \ldots \ldots \ldots \ldots \ldots \ldots \ldots \ldots \ldots$ & 3.03 & 3.15 & 3.27 & 3.73 & 3.72 & 3.70 \\
\hline Liquefied Petroleum Gas . . . . . . . . . . . . . . . . . & 2.28 & 2.35 & 2.39 & 2.43 & 2.43 & 2.37 \\
\hline Motor Gasoline $^{2} \ldots \ldots \ldots \ldots \ldots \ldots \ldots \ldots$ & 14.33 & 14.47 & 14.80 & 15.72 & 15.62 & 15.27 \\
\hline Petrochemical Feedstocks $\ldots \ldots \ldots \ldots \ldots \ldots \ldots \ldots \ldots$ & 1.19 & 1.25 & 1.25 & 1.27 & 1.27 & 1.24 \\
\hline Residual Fuel $\ldots \ldots \ldots \ldots \ldots \ldots \ldots \ldots \ldots$ & 1.54 & 1.55 & 1.59 & 1.76 & 1.73 & 1.70 \\
\hline Other Petroleum ${ }^{12} \ldots \ldots \ldots \ldots \ldots \ldots \ldots$ & 3.88 & 4.09 & 4.12 & 4.29 & 4.28 & 4.21 \\
\hline 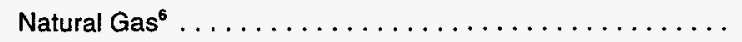 & 17.80 & 18.05 & 18.48 & 19.91 & 19.59 & 19.10 \\
\hline Metallurgical Coal . . . . . . . . . . . . . . . . . . & 0.84 & 0.84 & 0.81 & 0.88 & 0.71 & 0.68 \\
\hline Steam Coal $\ldots \ldots \ldots \ldots \ldots \ldots \ldots \ldots \ldots \ldots \ldots$ & 1.80 & 1.82 & 1.81 & 2.12 & 1.86 & 1.70 \\
\hline Net Coal Coke imports $\ldots \ldots \ldots \ldots \ldots \ldots$ & 0.02 & 0.02 & 0.02 & 0.03 & 0.03 & 0.02 \\
\hline Renewable Energy ${ }^{13} \ldots \ldots \ldots \ldots \ldots \ldots$ & 2.65 & 2.72 & 2.74 & 2.95 & 2.94 & 2.92 \\
\hline Methanol $^{11} \ldots \ldots \ldots \ldots \ldots \ldots \ldots \ldots \ldots \ldots$ & 0.00 & 0.00 & 0.00 & 0.00 & 0.00 & 0.00 \\
\hline Liquid Hydrogen $\ldots \ldots \ldots \ldots \ldots \ldots \ldots \ldots$ & 0.00 & 0.00 & 0.00 & 0.00 & 0.00 & 0.00 \\
\hline Electricity $\ldots \ldots \ldots \ldots \ldots \ldots \ldots \ldots \ldots \ldots$ & 9.76 & 9.97 & 10.15 & 10.75 & 10.70 & 10.21 \\
\hline Delivered Energy $\ldots \ldots \ldots \ldots \ldots \ldots \ldots \ldots \ldots \ldots \ldots$ & 65.62 & 66.99 & 68.16 & 73.09 & 72.03 & 70.00 \\
\hline Electricity Related Losses . . . . . . . . . . . . . . . . . & 21.76 & 22.15 & 22.44 & 23.16 & 23.03 & 21.87 \\
\hline 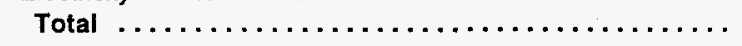 & 87.38 & 89.14 & 90.60 & 96.25 & 95.07 & 91.88 \\
\hline \multicolumn{7}{|l|}{ Electric Generators ${ }^{14}$} \\
\hline Distillate Fuel .. & 0.13 & 0.10 & 0.12 & 0.09 & 0.08 & 0.05 \\
\hline Residual Fuel . . . . . . . . . . . . & 0.95 & 0.89 & 0.61 & 0.62 & 0.60 & 0.45 \\
\hline$\ldots \ldots \ldots \ldots \ldots \ldots \ldots \ldots$ & 3.00 & 3.32 & 3.47 & 3.53 & 3.41 & 2.50 \\
\hline Steam Coal $\ldots \ldots \ldots \ldots \ldots \ldots \ldots \ldots \ldots \ldots$ & 16.89 & 16.97 & 17.02 & 18.10 & 18.07 & 17.51 \\
\hline$\ldots \ldots \ldots \ldots \ldots \ldots \ldots$ & 6.52 & 6.84 & 7.02 & 7.09 & 7.09 & 7.09 \\
\hline Renewable Energy $^{15} \ldots \ldots \ldots \ldots \ldots \ldots \ldots$ & 3.74 & 3.55 & 3.94 & 4.07 & 4.07 & 4.07 \\
\hline Electricity Imporis $\ldots \ldots \ldots \ldots \ldots \ldots \ldots \ldots \ldots$ & 0.29 & 0.46 & 0.40 & 0.42 & 0.42 & 0.42 \\
\hline Total $\ldots \ldots \ldots \ldots \ldots \ldots \ldots \ldots \ldots \ldots$ & 31.52 & 32.12 & 32.59 & 33.91 & 33.74 & 32.09 \\
\hline
\end{tabular}


Table B2. Energy Consumption by Sector and Source (Continued) (Quadrillion Btu per Year, Unless Otherwise Noted)

\begin{tabular}{c|c|c|c|c|c|c|c|c|c|c|}
\hline \multicolumn{9}{c|}{ Projections } & \multicolumn{2}{c|}{$\begin{array}{c}\text { Annual Growth } \\
1994-2015 \text { (percent) }\end{array}$} \\
\hline $\begin{array}{c}\text { Slow } \\
\text { Technology }\end{array}$ & Reference & $\begin{array}{c}\text { Rapid } \\
\text { Technology }\end{array}$ & $\begin{array}{c}\text { Slow } \\
\text { Technology }\end{array}$ & $\begin{array}{c}\text { 2010 } \\
\text { Reference }\end{array}$ & $\begin{array}{c}\text { Rapid } \\
\text { Technology }\end{array}$ & $\begin{array}{c}\text { Slow } \\
\text { Technology }\end{array}$ & Reference & $\begin{array}{c}\text { Rapid } \\
\text { Technology }\end{array}$ & $\begin{array}{c}\text { Slow } \\
\text { Technology }\end{array}$ & $\begin{array}{c}\text { Rapid } \\
\text { Reference }\end{array}$ \\
\hline
\end{tabular}

\begin{tabular}{|c|c|c|c|c|c|c|c|c|c|c|}
\hline 5.10 & 5.01 & 4.49 & 5.51 & 5.39 & 4.58 & 5.94 & 5.84 & 4.69 & 1.7 & 1.6 \\
\hline 4.19 & 4.13 & 4.05 & 4.55 & 4.43 & 4.27 & 4.88 & 4.69 & 4.41 & 2.1 & 1.9 \\
\hline 16.50 & 15.97 & 15.26 & 17.20 & 16.16 & 15.01 & 17.59 & 15.98 & 14.44 & 1.0 & 0.6 \\
\hline 1.21 & 1.22 & 1.21 & 1.36 & 1.37 & 1.36 & 1.49 & 1.51 & 1.50 & 2.4 & 2.5 \\
\hline 0.03 & 0.03 & 0.03 & 0.09 & 0.08 & 0.07 & 0.16 & 0.14 & 0.12 & 9.2 & 8.5 \\
\hline 0.23 & 0.23 & 0.23 & 0.23 & 0.23 & 0.23 & 0.24 & 0.24 & 0.24 & 0.9 & 0.9 \\
\hline 27.26 & 26.57 & 25.26 & 28.94 & 27.67 & 25.52 & 30.31 & 28.40 & 25.41 & 1.4 & 1.1 \\
\hline 0.71 & 0.71 & 0.65 & 0.78 & 0.76 & 0.68 & 0.85 & 0.86 & 0.77 & 1.2 & 1.3 \\
\hline 0.03 & 0.03 & 0.03 & 0.13 & 0.12 & 0.10 & 0.25 & 0.22 & 0.18 & 18.9 & 18.1 \\
\hline 0.06 & 0.05 & 0.05 & 0.12 & 0.11 & 0.10 & 0.19 & 0.16 & 0.14 & 26.3 & 25.4 \\
\hline 0.04 & 0.04 & 0.04 & 0.10 & 0.09 & 0.08 & 0.14 & 0.13 & 0.12 & 24.1 & 23.7 \\
\hline 0.00 & 0.00 & 0.00 & 0.00 & 0.00 & 0.00 & 0.00 & 0.00 & 0.00 & 56.9 & 56.8 \\
\hline 0.07 & 0.07 & $0.06^{\circ}$ & 0.12 & 0.11 & 0.10 & 0.15 & 0.14 & 0.12 & 10.1 & 9.5 \\
\hline 28.18 & 27.47 & 26.09 & 30.19 & 28.86 & 26.59 & 31.89 & 29.90 & 26.74 & 1.5 & 1.2 \\
\hline 0.15 & 0.14 & 0.13 & 0.25 & 0.23 & 0.20 & 0.30 & 0.26 & 0.24 & 9.4 & 8.8 \\
\hline 28.33 & 27.62 & 26.22 & 30.44 & 29.09 & 26.79 & 32.19 & 30.17 & 26.98 & 1.5 & 1.2 \\
\hline
\end{tabular}

\begin{tabular}{|c|c|c|c|c|c|c|c|c|c|c|c|}
\hline 7.66 & 7.52 & 6.89 & 8.10 & 7.93 & 6.84 & 8.80 & 8.42 & 6.82 & 1.4 & 1.2 & 0.1 \\
\hline 0.09 & 0.09 & 0.09 & 0.09 & 0.09 & 0.09 & 0.09 & 0.09 & 0.09 & -0.2 & -0.3 & -0.4 \\
\hline 4.19 & 4.13 & 4.05 & 4.55 & 4.43 & 4.27 & 4.88 & 4.69 & 4.41 & 2.1 & 1.9 & 1.6 \\
\hline 2.55 & 2.56 & 2.33 & 2.69 & 2.68 & 2.21 & 2.86 & 2.83 & 2.12 & 0.9 & 0.9 & -0.5 \\
\hline 16.79 & 16.25 & 15.52 & 17.50 & 16.46 & 15.26 & 17.89 & 16.28 & 14.66 & 1.0 & 0.6 & 0.1 \\
\hline 1.36 & 1.36 & 1.22 & 1.42 & 1.42 & 1.12 & 1.48 & 1.48 & 0.98 & 0.8 & 0.8 & -1.2 \\
\hline 1.94 & 1.89 & 1.83 & 2.12 & 2.05 & 1.90 & 2.28 & 2.21 & 2.09 & 1.9 & 1.7 & 1.4 \\
\hline 4.46 & 4.37 & 4.09 & 4.63 & 4.44 & 3.89 & 4.82 & 4.55 & 3.69 & 0.8 & 0.5 & -0.5 \\
\hline 20.98 & 20.50 & 19.51 & 21.78 & 21.35 & 19.90 & 22.17 & 22.06 & 20.03 & 1.0 & 1.0 & 0.5 \\
\hline 0.84 & 0.62 & 0.56 & 0.81 & 0.55 & 0.47 & 0.79 & 0.48 & 0.39 & -0.3 & -2.6 & -3.6 \\
\hline 2.41 & 1.90 & 1.52 & 2.85 & 1.97 & 1.42 & 3.41 & 2.10 & 1.38 & 3.0 & 0.7 & -1.3 \\
\hline 0.04 & 0.04 & 0.02 & 0.04 & 0.04 & 0.02 & 0.04 & 0.04 & 0.02 & 2.5 & 2.5 & -1.4 \\
\hline 3.22 & 3.20 & 3.17 & 3.45 & 3.41 & 3.35 & 3.68 & 3.62 & 3.53 & 1.5 & 1.4 & 1.3 \\
\hline 0.04 & 0.04 & 0.04 & 0.10 & 0.09 & 0.08 & 0.14 & 0.13 & 0.12 & 24.1 & 23.7 & 23.2 \\
\hline 0.00 & 0.00 & 0.00 & 0.00 & 0.00 & 0.00 & 0.00 & 0.00 & 0.00 & 56.9 & 56.8 & 57.7 \\
\hline 11.64 & 11.47 & 10.60 & 12.62 & 12.30 & 11.04 & 13.75 & 13.27 & 11.62 & 1.5 & 1.4 & 0.7 \\
\hline 78.21 & 75.94 & 71.44 & 82.75 & 79.19 & 71.87 & 87.08 & 82.25 & 71.95 & 1.3 & 1.0 & 0.3 \\
\hline 24.84 & 24.44 & 22.41 & 26.11 & 25.50 & 23.08 & 26.64 & 25.77 & 23.52 & 0.9 & 0.7 & 0.3 \\
\hline 103.04 & 100.38 & 93.85 & 108.86 & 104.69 & 94.95 & 113.72 & 108.02 & 95.46 & 1.2 & 0.9 & 0.3 \\
\hline 0.14 & 0.13 & 0.06 & 0.19 & 0.19 & 0.11 & 0.28 & 0.24 & 0.21 & 5.3 & 4.2 & 4.4 \\
\hline 0.90 & 0.83 & 0.53 & 1.10 & 1.00 & 0.67 & 1.31 & 0.90 & 0.80 & 1.8 & 0.0 & -0.6 \\
\hline 4.46 & 4.29 & 2.73 & 5.86 & 5.41 & 3.36 & 7.25 & 7.46 & 5.34 & 3.8 & 3.9 & 2.3 \\
\hline 19.51 & 19.27 & 18.35 & 20.32 & 19.99 & 18.93 & 21.79 & 20.64 & 19.45 & 1.2 & 0.9 & 0.7 \\
\hline 6.93 & 6.93 & 6.93 & 6.52 & 6.52 & 6.52 & 4.63 & 4.63 & 4.63 & -1.8 & -1.8 & -1.8 \\
\hline 4.24 & 4.19 & 4.12 & 4.41 & 4.38 & 4.22 & 4.86 & 4.90 & 4.44 & 1.5 & 1.6 & 1.1 \\
\hline 0.29 & 0.29 & 0.29 & 0.31 & 0.32 & 0.31 & 0.26 & 0.26 & 0.26 & -2.7 & -2.7 & -2.6 \\
\hline 36.47 & 35.92 & 33.01 & 38.73 & 37.80 & 34.12 & 40.39 & 39.04 & 35.14 & 1.1 & 0.9 & 0.4 \\
\hline
\end{tabular}


Table B2. Energy Consumption by Sector and Source (Continued)

(Quadrillion Btu per Year, Unless Otherwise Noted)

\begin{tabular}{|c|c|c|c|c|c|c|}
\hline \multirow[b]{3}{*}{ Sector and Source } & \multirow[b]{3}{*}{1993} & \multirow[b]{3}{*}{1994} & \multirow[b]{3}{*}{1995} & \multicolumn{3}{|c|}{ Projections } \\
\hline & & & & \multicolumn{3}{|c|}{2000} \\
\hline & & & & $\begin{array}{c}\text { Slow } \\
\text { Technology } \\
\end{array}$ & Reference & $\begin{array}{c}\text { Rapid } \\
\text { Technology }\end{array}$ \\
\hline \multicolumn{7}{|l|}{ Total Energy Consumption } \\
\hline 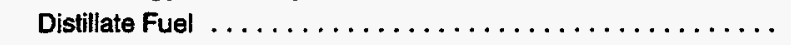 & 6.52 & 6.71 & 6.75 & 7.23 & 7.14 & 6.85 \\
\hline 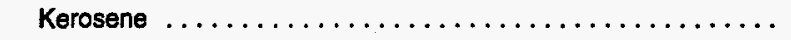 & 0.10 & 0.09 & 0.09 & 0.09 & 0.09 & 0.09 \\
\hline 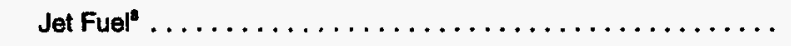 & 3.03 & 3.15 & 3.27 & 3.73 & 3.72 & 3.70 \\
\hline Liquefied Petroleum Gas . . . . . . . . . . . . . . . . . & 2.28 & 2.35 & 2.39 & 2.43 & 2.43 & 2.37 \\
\hline Motor Gasoline ${ }^{2}$ & 14.33 & 14.47 & 14.80 & 15.72 & 15.62 & 15.27 \\
\hline Petrochemical Feedstocks $\ldots \ldots \ldots \ldots \ldots \ldots \ldots \ldots$ & 1.19 & 1.25 & 1.25 & 1.27 & 1.27 & 1.24 \\
\hline Residual Fuel $\ldots \ldots \ldots \ldots \ldots \ldots \ldots \ldots \ldots \ldots \ldots \ldots$ & 2.49 & 2.44 & 2.20 & 2.38 & 2.33 & 2.14 \\
\hline Other Petroleum ${ }^{12} \ldots \ldots \ldots \ldots \ldots \ldots \ldots \ldots \ldots \ldots \ldots$ & 3.88 & 4.09 & 4.12 & 4.29 & 4.28 & 4.21 \\
\hline 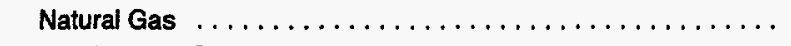 & 20.80 & 21.36 & 21.95 & 23.44 & 23.00 & 21.60 \\
\hline Metallurgical Coal $\ldots \ldots \ldots \ldots \ldots \ldots \ldots \ldots \ldots$ & 0.84 & 0.84 & 0.81 & 0.88 & 0.71 & 0.68 \\
\hline 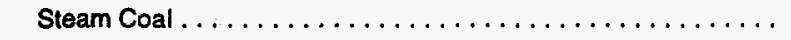 & 18.69 & 18.79 & 18.83 & 20.22 & 19.93 & 19.21 \\
\hline Net Coal Coke Imports $\ldots \ldots \ldots \ldots \ldots \ldots \ldots \ldots \ldots$ & 0.02 & 0.02 & 0.02 & 0.03 & 0.03 & 0.02 \\
\hline Nuclear Power $\ldots \ldots \ldots \ldots \ldots \ldots \ldots \ldots \ldots \ldots$ & 6.52 & 6.84 & 7.02 & 7.09 & 7.09 & 7.09 \\
\hline Renewable Energy ${ }^{16}$ & 6.40 & 6.27 & 6.68 & 7.02 & 7.01 & 7.00 \\
\hline Methanol $\ldots \ldots \ldots \ldots \ldots \ldots \ldots \ldots \ldots \ldots \ldots \ldots \ldots$ & 0.00 & 0.00 & 0.00 & 0.00 & 0.00 & 0.00 \\
\hline Liquid Hydrogen $\ldots \ldots \ldots \ldots \ldots \ldots \ldots \ldots \ldots \ldots \ldots$ & 0.00 & 0.00 & 0.00 & 0.00 & 0.00 & 0.00 \\
\hline Electricity Imports $\ldots \ldots \ldots \ldots \ldots \ldots \ldots \ldots \ldots \ldots \ldots \ldots$ & 0.29 & 0.46 & 0.40 & 0.42 & 0.42 & 0.42 \\
\hline 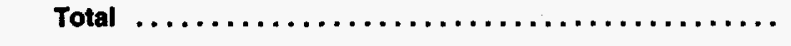 & 87.38 & 89.14 & 90.60 & 96.25 & 95.07 & 91.88 \\
\hline \multicolumn{7}{|l|}{ Energy Use and Related Statistics } \\
\hline Delivered Energy Use $\ldots \ldots \ldots \ldots \ldots \ldots \ldots \ldots \ldots$ & 65.62 & 66.99 & 68.16 & 73.09 & 72.03 & 70.00 \\
\hline Total Energy Use $\ldots \ldots \ldots \ldots \ldots \ldots \ldots \ldots \ldots \ldots$ & 87.39 & 89.14 & 90.60 & 96.26 & 95.07 & 91.89 \\
\hline Population (millions) $\ldots \ldots \ldots \ldots \ldots \ldots \ldots \ldots \ldots \ldots$ & 258.43 & 261.03 & 263.58 & 275.62 & 275.62 & 275.62 \\
\hline
\end{tabular}


Table B2. Energy Consumption by Sector and Source (Continued) (Quadrillion Btu per Year, Unless Otherwise Noted)

\begin{tabular}{|c|c|c|c|c|c|c|c|c|c|c|c|}
\hline \multicolumn{9}{|c|}{ Projections } & \multirow{2}{*}{\multicolumn{3}{|c|}{$\begin{array}{l}\text { Annual Growth } \\
1994-2015 \text { (percent) }\end{array}$}} \\
\hline \multicolumn{3}{|c|}{2005} & \multicolumn{3}{|c|}{2010} & \multicolumn{3}{|c|}{2015} & & & \\
\hline $\begin{array}{c}\text { Slow } \\
\text { Technology }\end{array}$ & Reference & $\begin{array}{c}\text { Rapid } \\
\text { Technology }\end{array}$ & $\begin{array}{c}\text { Slow } \\
\text { Technology }\end{array}$ & Reference & $\begin{array}{c}\text { Rapid } \\
\text { Technology }\end{array}$ & $\begin{array}{c}\text { Slow } \\
\text { Technology }\end{array}$ & Reference & $\begin{array}{c}\text { Rapid } \\
\text { Technology }\end{array}$ & $\begin{array}{c}\text { Slow } \\
\text { Technology }\end{array}$ & Reference & $\begin{array}{c}\text { Rapid } \\
\text { Technology }\end{array}$ \\
\hline
\end{tabular}

\begin{tabular}{|c|c|c|c|c|c|c|c|c|c|c|c|}
\hline 7.80 & 7.65 & 6.95 & 8.30 & 8.11 & 6.95 & 9.08 & 8.66 & 7.02 & 1.5 & 1.2 & 0.2 \\
\hline 0.09 & 0.09 & 0.09 & 0.09 & 0.09 & 0.09 & 0.09 & 0.09 & 0.09 & -0.2 & -0.3 & -0.4 \\
\hline 4.19 & 4.13 & 4.05 & 4.55 & 4.43 & 4.27 & 4.88 & 4.69 & 4.41 & 2.1 & 1.9 & 1.6 \\
\hline 2.55 & 2.56 & 2.33 & 2.69 & 2.68 & 2.21 & 2.86 & 2.83 & 2.12 & 0.9 & 0.9 & -0.5 \\
\hline 16.79 & 16.25 & 15.52 & 17.50 & 16.46 & 15.26 & 17.89 & 16.28 & 14.66 & 1.0 & 0.6 & 0.1 \\
\hline 1.36 & 1.36 & 1.22 & 1.42 & 1.42 & 1.12 & 1.48 & 1.48 & 0.98 & 0.8 & 0.8 & -1.2 \\
\hline 2.84 & 2.72 & 2.36 & 3.22 & 3.05 & 2.58 & 3.59 & 3.11 & 2.89 & 1.8 & 1.2 & 0.8 \\
\hline 4.46 & 4.37 & 4.09 & 4.63 & 4.44 & 3.89 & 4.82 & 4.55 & 3.69 & 0.8 & 0.5 & -0.5 \\
\hline 25.45 & 24.79 & 22.24 & 27.65 & 26.76 & 23.26 & 29.42 & 29.52 & 25.37 & 1.5 & 1.6 & 0.8 \\
\hline 0.84 & 0.62 & 0.56 & 0.81 & 0.55 & 0.47 & 0.79 & 0.48 & 0.39 & -0.3 & -2.6 & -3.6 \\
\hline 21.92 & 21.17 & 19.87 & 23.18 & 21.96 & 20.35 & 25.20 & 22.75 & 20.83 & 1.4 & 0.9 & 0.5 \\
\hline 0.04 & 0.04 & 0.02 & 0.04 & 0.04 & 0.02 & 0.04 & 0.04 & 0.02 & 2.5 & 2.5 & -1.4 \\
\hline 6.93 & 6.93 & 6.93 & 6.52 & 6.52 & 6.52 & 4.63 & 4.63 & 4.63 & -1.8 & -1.8 & -1.8 \\
\hline 7.46 & 7.38 & 7.28 & 7.86 & 7.78 & 7.58 & 8.54 & 8.52 & 7.98 & 1.5 & 1.5 & 1.2 \\
\hline 0.04 & 0.04 & 0.04 & 0.10 & 0.09 & 0.08 & 0.14 & 0.13 & 0.12 & 24.1 & 23.7 & 23.2 \\
\hline 0.00 & 0.00 & 0.00 & 0.00 & 0.00 & 0.00 & 0.00 & 0.00 & 0.00 & 56.9 & 56.8 & 57.7 \\
\hline 0.29 & 0.29 & 0.29 & 0.31 & 0.32 & 0.31 & 0.26 & 0.26 & 0.26 & -2.7 & -2.7 & -2.6 \\
\hline 103.04 & 100.38 & 93.85 & 108.86 & 104.69 & 94.95 & 113.72 & 108.02 & 95.46 & 1.2 & 0.9 & 0.3 \\
\hline
\end{tabular}

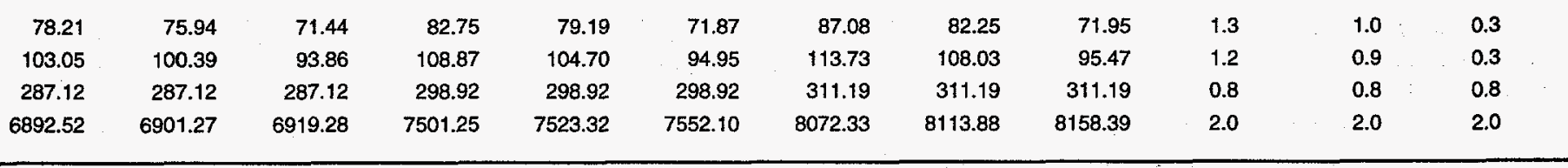

'Includes wood use for residential heating and cooking. See Table 18 for estimates of nonmarketed renewable energy consumption for geothermal heat pumps and solar thermal hot water heating.

${ }^{2}$ Includes ethanol (blends of 10 percent or less) and ethers blended into gasoline.

Includes commercial sector electricity cogenerated using wood and wood waste, municipal solid waste, and other biomass. See Table 18 for estimates of nonmarketed renewable energy consumption for solar thermal hot water heating.

4 Fuel consumption includes consumption for cogeneration.

Includes petroleum coke, asphalt, road oil, lubricants, still gas, and miscellaneous petroleum products.

includes lease and plant fuel.

IIncludes consumption of renewable energy from water, wood and wood waste, municipal solid waste, and other biomass.

Includes naphtha and kerosene type.

Includes aviation gas and lubricants.

${ }^{10}$ E85 is 85 percent ethanol (renewable) and 15 percent motor gasoline(nonrenewable).

"Only M85 (85 percent methanol).

${ }^{12}$ Includes unfinished oils, natural gasoline, motor gasoline blending compounds, aviation gasoline, lubricants, still gas, asphalt, road oil, petroleum coke, and miscellaneous petroleum products.

${ }^{13}$ Includes electricity generated for sale to electric utilities and for self use from renewable sources, and non-electric energy from renewable sources. Excludes nonmarketed renewable energy consumption from geothermal heat pumps and solar thermal hot water heaters.

${ }^{14}$ Includes consumption of energy by all electric power generators except cogenerators, which produce electricity as a by-product of other processes.

${ }^{15} \mathrm{Includes}$ hydroelectric, geothermal, wood and wood waste, municipal solid waste, other biomass, wind, photovoltaic and solar thermal sources.

IIncludes hydroelectric, geothemal, wood and wood waste, municipal solid waste, other biomass, wind, photovoltaic and solar thermal sources. Excludes nonmarketed renewable energy consumption from ground source heat pumps and solar thermal hot water heaters.

Btu $=$ British thermal unit.

Note: Totals may not equal sum of components due to independent rounding. Figures for 1993 and 1994 may differ from published data due to internal conversion factors. Consumption values of 0.00 are values that round to 0.00 , because they are less than 0.005 .

Sources: 1993 natural gas lease, plant, and pipeline fuel values: Energy Information Administration (EIA), Natural Gas Annual 1993, DOE/EIA-0131(93) (Washington, DC, October 1994) 1994 natural gas lease, plant, and pipeline fuel values: EIA, Natural Gas Monthly, DOE/EIA-0130(95/6) (Washington, DC, June 1995). 1994 transportation sector compressed natural gas consumption: EIA, AEO96 National Energy Modeling System runs TECH01.D031396A (Slow Technology), AEO96B.D101995C (reference), and TECH04.D031396B (Rapid Technology). 1993 and 1994 electric utility fuel consumption: ElA, Electric Power Annual, Volume l, DOE/EIA-0348(94)/1 (Washington, DC, July 1995). 1993 and 1994 nonutility consumption estimates: EIA Form 867, "Annual Nonutility Power Producer Report." Other 1993 values: EIA, State Energy Data Report 1993, DOE/EIA-0214(93) (Washington, DC July 1995) and Office of Coal, Nuclear, Electric, and Attemative Fuels estimates. Other 1994 values: ElA, Short-Term Energy Outlook, DOE/EIA-0202(95/3Q) (Washington, DC, August 1995). Projections: EIA, AEO96 National Energy Modeling System runs TECH01.D031396A (Slow Technology), AEO96B.D101995C (reference), and TECHO4.D031396B (Rapid Technology). 


\begin{tabular}{|c|c|c|c|c|c|c|}
\hline \multirow[b]{3}{*}{ Sector and Source } & \multirow[b]{3}{*}{1993} & \multirow[b]{3}{*}{1994} & \multirow[b]{3}{*}{1995} & \multicolumn{3}{|c|}{ Projections } \\
\hline & & & & \multicolumn{3}{|c|}{2000} \\
\hline & & & & $\begin{array}{c}\text { Slow } \\
\text { Technology }\end{array}$ & Reference & $\begin{array}{c}\text { Rapid } \\
\text { Technology }\end{array}$ \\
\hline Residential $\ldots \ldots \ldots \ldots \ldots \ldots \ldots \ldots \ldots \ldots \ldots \ldots \ldots$ & 12.73 & 12.80 & 12.88 & 13.11 & 13.04 & 12.81 \\
\hline Primary Energy . . . . . . . . . . . . . . . . . . & 6.42 & 6.46 & 6.36 & 6.63 & 6.58 & 6.46 \\
\hline Petroleum Products $\ldots \ldots \ldots \ldots \ldots \ldots \ldots \ldots$ & 7.92 & 7.62 & 8.15 & 8.95 & 8.84 & 8.71 \\
\hline Distiltate Fuel $\ldots \ldots \ldots \ldots \ldots \ldots \ldots \ldots \ldots$ & 6.69 & 6.33 & 6.82 & 7.59 & 7.48 & 7.30 \\
\hline Liquefied Petroleum Gas . . . . . . . . . . . . . . . . . . . . & 10.76 & 10.44 & 10.99 & 11.99 & 11.87 & 11.85 \\
\hline Natural Gas $\ldots \ldots \ldots \ldots \ldots \ldots \ldots \ldots \ldots$ & 6.07 & 6.21 & 5.95 & 6.10 & 6.08 & 5.94 \\
\hline Electricity $\ldots \ldots \ldots \ldots \ldots \ldots \ldots \ldots \ldots \ldots$ & 24.87 & 24.67 & 24.74 & 24.69 & 24.49 & 24.27 \\
\hline 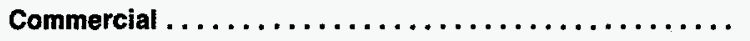 & 13.05 & 13.29 & 13.15 & 13.08 & 13.00 & 12.70 \\
\hline Primary Energy . . . . . . . . . . . . . . . . . . . & 4.99 & 5.10 & 4.83 & 5.17 & 5.13 & 4.98 \\
\hline Petroleum Products $\ldots \ldots \ldots \ldots \ldots \ldots \ldots \ldots$ & 4.90 & 4.80 & 4.90 & 5.64 & 5.54 & 5.34 \\
\hline Distillate Fuel $\ldots \ldots \ldots \ldots \ldots \ldots \ldots \ldots \ldots \ldots$ & 4.71 & 4.44 & 4.65 & 5.41 & 5.30 & 5.10 \\
\hline Residual Fuel $\ldots \ldots \ldots \ldots \ldots \ldots \ldots \ldots \ldots \ldots$ & 2.88 & 2.85 & 2.65 & 3.10 & 3.04 & 2.89 \\
\hline Natural Gas ${ }^{\dagger} \ldots \ldots \ldots \ldots \ldots \ldots \ldots \ldots \ldots$ & 5.11 & 5.28 & 4.91 & 5.16 & 5.14 & 5.00 \\
\hline Electricity $\ldots \ldots \ldots \ldots \ldots \ldots \ldots \ldots \ldots \ldots$ & 23.24 & 23.30 & 23.15 & 22.56 & 22.43 & 22.50 \\
\hline 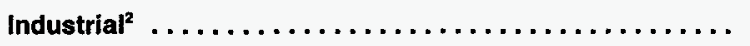 & 5.02 & 5.01 & 4.70 & 5.01 & 5.02 & 4.92 \\
\hline Primary Energy $\ldots \ldots \ldots \ldots \ldots \ldots \ldots \ldots \ldots$ & 3.29 & 3.31 & 3.01 & 3.43 & 3.41 & 3.28 \\
\hline Petroleum Products $\ldots \ldots \ldots \ldots \ldots \ldots \ldots \ldots \ldots$ & 4.24 & 4.50 & 4.10 & 4.85 & 4.77 & 4.64 \\
\hline Distillate Fuel $\ldots \ldots \ldots \ldots \ldots \ldots \ldots \ldots \ldots \ldots$ & 4.87 & 4.59 & 4.64 & 5.44 & 5.33 & 5.13 \\
\hline Liquefied Petroleum Gas $\ldots \ldots \ldots \ldots \ldots \ldots \ldots \ldots \ldots$ & 4.87 & 6.11 & 5.84 & 6.76 & 6.66 & 6.66 \\
\hline Residual Fuel $\ldots \ldots \ldots \ldots \ldots \ldots \ldots \ldots \ldots \ldots$ & 2.44 & 2.50 & 2.54 & 2.98 & 2.92 & 2.78 \\
\hline Natural $\mathrm{Gas}^{3} \ldots \ldots \ldots \ldots \ldots \ldots \ldots \ldots \ldots$ & 2.82 & 2.56 & 2.30 & 2.61 & 2.57 & 2.42 \\
\hline Metallurgical Coal $\ldots \ldots \ldots \ldots \ldots \ldots \ldots \ldots \ldots$ & 1.77 & 1.76 & 1.77 & 1.74 & 1.63 & 1.57 \\
\hline Steam Coal . . . . . . . . . . . . . . . . . . . . . . & 1.45 & 1.45 & 1.38 & 1.35 & 1.30 & 1.23 \\
\hline Electricity $\ldots \ldots \ldots \ldots \ldots \ldots \ldots \ldots \ldots \ldots$ & 14.76 & 14.75 & 14.55 & 14.40 & 14.33 & 14.30 \\
\hline Transportation $\ldots \ldots \ldots \ldots \ldots \ldots \ldots \ldots \ldots \ldots$ & 7.97 & 7.74 & $\mathbf{8 . 3 0}$ & 9.17 & 9.04 & $\mathbf{8 . 7 6}$ \\
\hline Primary Energy $\ldots \ldots \ldots \ldots \ldots \ldots \ldots \ldots \ldots \ldots$ & 7.96 & 7.74 & 8.30 & 9.16 & 9.03 & 8.75 \\
\hline Petroleum Products $\ldots \ldots \ldots \ldots \ldots \ldots \ldots \ldots \ldots$ & 7.96 & 7.74 & 8.30 & 9.16 & 9.03 & 8.75 \\
\hline Distillate Fuel ${ }^{4} \ldots \ldots \ldots \ldots \ldots \ldots \ldots \ldots$ & 8.21 & 8.04 & 8.27 & 8.90 & 8.79 & $\mathbf{8 . 5 7}$ \\
\hline Jet Fuel ${ }^{5} \ldots \ldots \ldots \ldots \ldots \ldots \ldots \ldots \ldots \ldots \ldots$ & 4.38 & 3.95 & 4.29 & 5.46 & 5.35 & 5.13 \\
\hline Motor Gasoline $^{6} \ldots \ldots \ldots \ldots \ldots \ldots \ldots \ldots$ & 9.11 & 8.92 & 9.66 & 10.65 & 10.51 & 10.21 \\
\hline Residual Fuel $\ldots \ldots \ldots \ldots \ldots \ldots \ldots \ldots \ldots$ & 2.10 & 2.02 & 2.30 & 2.76 & 2.69 & 2.56 \\
\hline Natural Gas ${ }^{7} \ldots \ldots \ldots \ldots \ldots \ldots \ldots \ldots \ldots$ & 5.52 & 6.01 & 6.07 & 6.58 & 6.55 & 6.40 \\
\hline Electricity $\ldots \ldots \ldots \ldots \ldots \ldots \ldots \ldots \ldots \ldots$ & 14.64 & 14.80 & 14.81 & 15.19 & 15.24 & 15.41 \\
\hline Total End-Use Energy $\ldots \ldots \ldots \ldots \ldots \ldots \ldots \ldots \ldots$ & 8.24 & 8.16 & 8.23 & 8.66 & 8.62 & 8.41 \\
\hline Primary Energy $\ldots \ldots \ldots \ldots \ldots \ldots \ldots \ldots \ldots \ldots \ldots$ & 7.87 & 7.76 & 7.86 & 8.34 & 8.30 & 8.10 \\
\hline Electricity $\ldots \ldots \ldots \ldots \ldots \ldots \ldots \ldots \ldots \ldots$ & 20.89 & 20.85 & 20.77 & 20.53 & 20.39 & 20.28 \\
\hline \multicolumn{7}{|l|}{ Electric Generators ${ }^{8}$} \\
\hline Fossil Fuel Average $\ldots \ldots \ldots \ldots \ldots \ldots \ldots \ldots$ & 1.62 & 1.56 & 1.50 & 1.52 & 1.46 & 1.34 \\
\hline Petroleum Products $\ldots \ldots \ldots \ldots \ldots \ldots \ldots \ldots$ & 2.83 & 2.76 & 3.03 & 3.36 & 3.27 & 3.11 \\
\hline Distillate Fuel $\ldots \ldots \ldots \ldots \ldots \ldots \ldots \ldots \ldots$ & 4.23 & 3.96 & 4.21 & 5.06 & 4.95 & 4.78 \\
\hline Residual Fuel $\ldots \ldots \ldots \ldots \ldots \ldots \ldots \ldots \ldots$ & 2.64 & 2.62 & 2.79 & 3.11 & 3.04 & 2.91 \\
\hline Natural Gas $\ldots \ldots \ldots \ldots \ldots \ldots \ldots \ldots \ldots$ & 2.55 & 2.19 & 2.04 & 2.24 & 2.19 & 2.01 \\
\hline Steam Coal $\ldots \ldots \ldots \ldots \ldots \ldots \ldots \ldots \ldots \ldots$ & 1.38 & 1.36 & 1.32 & 1.31 & 1.26 & 1.19 \\
\hline
\end{tabular}


Table B3. Energy Prices by Sector and Source (Continued) (1994 Dollars per Million Btu)

\begin{tabular}{c|c|c|c|c|c|c|c|c|c|c|}
\hline \multicolumn{9}{c|}{ Projections } & \multicolumn{2}{c|}{$\begin{array}{c}\text { Annual Growth } \\
1994-2015 \text { (percent) }\end{array}$} \\
\hline $\begin{array}{c}\text { Slow } \\
\text { Technology }\end{array}$ & $\begin{array}{c}2005 \\
\text { Reference }\end{array}$ & $\begin{array}{c}\text { Rapid } \\
\text { Technology }\end{array}$ & $\begin{array}{c}\text { Slow } \\
\text { Technology }\end{array}$ & $\begin{array}{c}\text { Rapid } \\
\text { Reference }\end{array}$ & $\begin{array}{c}\text { Slow } \\
\text { Technology }\end{array}$ & Rechnology & Reference & $\begin{array}{c}\text { Rapid } \\
\text { Technology }\end{array}$ & $\begin{array}{c}\text { Slow } \\
\text { Technology }\end{array}$ & $\begin{array}{c}\text { Rapid } \\
\text { Reference }\end{array}$ \\
\hline
\end{tabular}

\begin{tabular}{|c|c|c|c|c|c|c|c|c|c|c|}
\hline 13.66 & 13.35 & 12.81 & 14.64 & 13.65 & 12.72 & 16.14 & 14.31 & 12.89 & 1.1 & 0.5 \\
\hline 6.78 & 6.55 & 6.14 & 7.33 & 6.53 & 5.80 & 8.46 & 6.95 & 5.75 & 1.3 & 0.3 \\
\hline 9.43 & 9.28 & 8.83 & 10.00 & 9.63 & 8.93 & 10.43 & 9.77 & 8.52 & 1.5 & 1.2 \\
\hline 8.07 & 7.89 & 7.45 & 8.56 & 8.19 & 7.57 & 8.94 & 8.35 & 7.51 & 1.7 & 1.3 \\
\hline 12.59 & 12.46 & 11.99 & 13.52 & 13.03 & 12.13 & 14.23 & 13.26 & 10.88 & 1.5 & 1.1 \\
\hline 6.20 & 5.96 & 5.54 & 6.78 & 5.89 & 5.13 & 8.09 & 6.39 & 5.18 & 1.3 & 0.1 \\
\hline 25.15 & 24.62 & 24.13 & 25.93 & 24.63 & 23.70 & 27.00 & 24.72 & 23.23 & 0.4 & 0.0 \\
\hline 13.43 & 13.15 & 12.44 & 14.11 & 13.21 & 12.07 & 15.35 & 13.66 & 11.94 & 0.7 & 0.1 \\
\hline 5.35 & 5.14 & 4.72 & 5.92 & 5.17 & 4.46 & 7.01 & 5.59 & 4.49 & 1.5 & 0.4 \\
\hline 6.17 & 5.96 & 5.47 & 6.68 & 6.29 & 5.59 & 7.09 & 6.45 & 5.44 & 1.9 & 1.4 \\
\hline 5.88 & 5.69 & 5.22 & 6.35 & 5.99 & 5.34 & 6.74 & 6.13 & 5.26 & 2.0 & 1.5 \\
\hline 3.67 & 3.48 & 3.11 & 4.10 & 3.80 & 3.26 & 4.56 & 4.05 & 3.40 & 2.3 & 1.7 \\
\hline 5.28 & 5.06 & 4.64 & 5.88 & 5.03 & 4.28 & 7.15 & 5.53 & 4.37 & 1.5 & 0.2 \\
\hline 22.78 & 22.49 & 22.31 & 23.14 & 22.27 & 21.65 & 23.96 & 22.29 & 21.09 & 0.1 & -0.2 \\
\hline
\end{tabular}

\begin{tabular}{|c|c|c|c|c|c|c|c|c|c|c|}
\hline 5.31 & 5.25 & 4.92 & 5.78 & 5.42 & 4.83 & 6.41 & 5.64 & 4.68 & 1.2 & 0.6 \\
\hline 3.74 & 3.63 & 3.24 & 4.25 & 3.84 & 3.15 & 4.92 & 4.08 & 2.98 & 1.9 & 1.0 \\
\hline 5.32 & 5.18 & 4.78 & 5.84 & 5.52 & 4.85 & 6.29 & 5.61 & 4.36 & 1.6 & 1.1 \\
\hline 5.91 & 5.72 & 5.27 & 6.39 & 6.04 & 5.40 & 6.79 & 6.18 & 5.34 & 1.9 & 1.4 \\
\hline 7.21 & 7.13 & 6.70 & 7.94 & 7.50 & 6.54 & 8.54 & 7.59 & 5.24 & 1.6 & 1.0 \\
\hline 3.53 & 3.35 & 3.00 & 3.95 & 3.66 & 3.14 & 4.42 & 3.92 & 3.26 & 2.7 & 2.2 \\
\hline 2.88 & 2.64 & 2.19 & 3.59 & 2.76 & 2.02 & 4.79 & 3.20 & 2.14 & 3.0 & 1.1 \\
\hline 1.85 & 1.66 & 1.58 & 1.85 & 1.66 & 1.56 & 1.87 & 1.64 & 1.54 & 0.3 & -0.3 \\
\hline 1.45 & 1.33 & 1.21 & 1.48 & 1.33 & 1.19 & 1.50 & 1.31 & 1.19 & 0.2 & -0.5 \\
\hline 14.52 & 14.37 & 14.12 & 14.62 & 14.14 & 13.61 & 14.97 & 14.07 & 13.07 & 0.1 & -0.2 \\
\hline
\end{tabular}

\begin{tabular}{|c|c|c|c|c|c|c|c|c|c|c|c|}
\hline 9.68 & 9.35 & 8.65 & 10.10 & 9.47 & 8.51 & 10.29 & 9.37 & 8.10 & 1.4 & 0.9 & 0.2 \\
\hline 9.67 & 9.33 & 8.64 & 10.08 & 9.45 & 8.49 & 10.26 & 9.34 & 8.07 & 1.4 & 0.9 & 0.2 \\
\hline 9.67 & 9.33 & 8.64 & 10.09 & 9.46 & 8.50 & 10.28 & 9.36 & 8.08 & 1.4 & 0.9 & 0.2 \\
\hline 9.21 & 9.00 & 8.49 & 9.51 & 9.12 & 8.42 & 9.81 & 9.21 & 8.23 & 1.0 & 0.6 & 0.1 \\
\hline 6.10 & 5.88 & 5.35 & 6.64 & 6.17 & 5.51 & 7.13 & 6.36 & 5.40 & 2.8 & 2.3 & 1.5 \\
\hline 11.26 & 10.87 & 10.08 & 11.74 & 11.04 & 9.93 & 11.85 & 10.86 & 9.42 & 1.4 & 0.9 & 0.3 \\
\hline 3.31 & 3.12 & 2.78 & 3.74 & 3.44 & 2.93 & 4.23 & 3.72 & 3.06 & 3.6 & 3.0 & 2.0 \\
\hline 6.76 & 6.51 & 6.06 & 7.35 & 6.47 & 5.67 & 8.78 & 7.15 & 6.02 & 1.8 & 0.8 & 0.0 \\
\hline 15.49 & 15.56 & 15.70 & 15.49 & 15.51 & 15.55 & 15.47 & 15.43 & 15.17 & 0.2 & 0.2 & 0.1 \\
\hline
\end{tabular}

\begin{tabular}{|c|c|c|c|c|c|c|c|c|c|c|c|}
\hline 9.07 & 8.88 & 8.37 & 9.62 & 9.06 & 8.30 & 10.30 & 9.27 & 8.19 & 1.1 & 0.6 & 0.0 \\
\hline 8.74 & 8.56 & 8.06 & 9.25 & 8.71 & 7.98 & 9.80 & 8.84 & 7.81 & 1.1 & 0.6 & 0.0 \\
\hline 20.79 & 20.46 & 20.11 & 21.27 & 20.38 & 19.67 & 22.14 & 20.48 & 19.29 & 0.3 & -0.1 & -0.4 \\
\hline
\end{tabular}

$\begin{array}{rrrrrrrrrrrr}1.69 & 1.54 & 1.31 & 1.95 & 1.63 & 1.30 & 2.36 & 1.83 & 1.39 & 2.0 & 0.8 & -0.5 \\ 3.88 & 3.68 & 3.26 & 4.35 & 4.04 & 3.45 & 4.86 & 4.38 & 3.68 & 2.7 & 2.2 & 1.4 \\ 5.51 & 5.32 & 4.91 & 5.90 & 5.57 & 4.99 & 6.28 & 5.70 & 4.84 & 2.2 & 1.8 & 0.9 \\ 3.62 & 3.43 & 3.06 & 4.08 & 3.75 & 3.21 & 4.55 & 4.03 & 3.38 & 2.7 & 2.1 \\ 2.51 & 2.26 & 1.76 & 3.32 & 2.44 & 1.60 & 4.58 & 2.95 & 1.79 & 3.6 & 1.2 \\ 1.38 & 1.28 & 1.18 & 1.39 & 1.26 & 1.16 & 1.44 & 1.28 & 1.17 & 0.3 & -0.3 & -0.7\end{array}$


Table B3. Energy Prices by Sector and Source (Continued) (1994 Dollars per Million Btu)

\begin{tabular}{|c|c|c|c|c|c|c|}
\hline \multirow[b]{3}{*}{ Sector and Source } & \multirow[b]{3}{*}{1993} & \multirow[b]{3}{*}{1994} & \multirow[b]{3}{*}{1995} & \multicolumn{3}{|c|}{ Projections } \\
\hline & & & & \multicolumn{3}{|c|}{2000} \\
\hline & & & & $\begin{array}{c}\text { Slow } \\
\text { Technology } \\
\end{array}$ & Reference & $\begin{array}{c}\text { Rapid } \\
\text { Technology } \\
\end{array}$ \\
\hline \multicolumn{7}{|l|}{ Average Price to All Users ${ }^{\circ}$} \\
\hline Petroleum Products ....... & 6.88 & 6.77 & 7.11 & 7.97 & 7.86 & 7.64 \\
\hline Distillate Fuel ${ }^{4} \ldots \ldots \ldots \ldots \ldots \ldots \ldots \ldots \ldots$ & 7.11 & 6.94 & 7.17 & 7.91 & 7.79 & 7.57 \\
\hline Jet Fuel $\ldots \ldots \ldots \ldots \ldots \ldots \ldots \ldots \ldots \ldots \ldots$ & 4.38 & 3.95 & 4.29 & 5.46 & 5.35 & 5.13 \\
\hline Liquefied Petroleum Gas . . . . . . . . . . . . . . . . & 6.06 & 6.94 & 6.84 & 7.73 & 7.62 & 7.63 \\
\hline Motor Gasoline $^{6} \ldots \ldots \ldots \ldots \ldots \ldots \ldots$ & 9.11 & 8.92 & 9.64 & 10.63 & 10.49 & 10.18 \\
\hline Residual Fuel $\ldots \ldots \ldots \ldots \ldots \ldots \ldots \ldots \ldots \ldots \ldots$ & 2.42 & 2.39 & 2.51 & 2.92 & 2.86 & 2.71 \\
\hline Natural Gas $\ldots \ldots \ldots \ldots \ldots \ldots \ldots \ldots \ldots \ldots \ldots$ & 4.01 & 3.86 & 3.56 & 3.79 & 3.77 & 3.69 \\
\hline Coal $\ldots \ldots \ldots \ldots \ldots \ldots \ldots \ldots \ldots \ldots \ldots \ldots \ldots \ldots \ldots$ & 1.41 & 1.39 & 1.33 & 1.31 & 1.26 & 1.20 \\
\hline 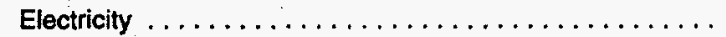 & 20.89 & 20.85 & 20.77 & 20.53 & 20.39 & 20.28 \\
\hline
\end{tabular}


Table B3. Energy Prices by Sector and Source (Continued) (1994 Dollars per Million Btu)

\begin{tabular}{|c|c|c|c|c|c|c|c|c|c|c|c|}
\hline \multicolumn{9}{|c|}{ Projections } & \multirow{2}{*}{\multicolumn{3}{|c|}{$\begin{array}{c}\text { Annual Growth } \\
\text { 1994-2015 (percent) }\end{array}$}} \\
\hline \multicolumn{3}{|c|}{2005} & \multicolumn{3}{|c|}{2010} & \multicolumn{3}{|c|}{2015} & & & \\
\hline $\begin{array}{c}\text { Slow } \\
\text { Technology }\end{array}$ & Reference & $\begin{array}{c}\text { Rapid } \\
\text { Technology }\end{array}$ & $\begin{array}{c}\text { Slow } \\
\text { Technology }\end{array}$ & Reference & $\begin{array}{c}\text { Rapid } \\
\text { Technology }\end{array}$ & $\begin{array}{c}\text { Slow } \\
\text { Technology }\end{array}$ & Reference & $\begin{array}{c}\text { Rapid } \\
\text { Technology }\end{array}$ & $\begin{array}{c}\text { Slow } \\
\text { Technology } \\
\end{array}$ & Reference & $\begin{array}{c}\text { Rapid } \\
\text { Technology }\end{array}$ \\
\hline 8.46 & 8.18 & 7.64 & 8.90 & 8.36 & 7.60 & 9.15 & 8.33 & 7.20 & 1.4 & 1.0 & 0.3 \\
\hline 8.29 & 8.09 & 7.59 & 8.67 & 8.28 & 7.62 & 8.96 & 8.41 & 7.48 & 1.2 & 0.9 & 0.4 \\
\hline 6.10 & 5.88 & 5.35 & 6.64 & 6.17 & 5.51 & 7.13 & 6.36 & 5.40 & 2.8 & 2.3 & 1.5 \\
\hline 8.15 & 8.04 & 7.66 & 8.97 & 8.48 & 7.69 & 9.67 & 8.65 & 6.55 & 1.6 & 1.1 & -0.3 \\
\hline 11.24 & 10.86 & 10.07 & 11.73 & 11.02 & 9.92 & 11.84 & 10.84 & 9.41 & 1.4 & 0.9 & 0.3 \\
\hline 3.47 & 3.28 & 2.91 & 3.91 & 3.60 & 3.05 & 4.40 & 3.86 & 3.20 & 2.9 & 2.3 & 1.4 \\
\hline 3.93 & 3.71 & 3.37 & 4.57 & 3.73 & 3.07 & 5.79 & 4.13 & 3.10 & 1.9 & 0.3 & -1.0 \\
\hline 1.39 & 1.28 & 1.18 & 1.41 & 1.27 & 1.17 & 1.45 & 1.28 & 1.17 & 0.2 & -0.4 & -0.8 \\
\hline 20.79 & 20.46 & 20.11 & 21.27 & 20.38 & 19.67 & 22.14 & 20.48 & 19.29 & 0.3 & -0.1 & -0.4 \\
\hline
\end{tabular}

${ }^{1}$ Excludes independent power producers.

${ }^{2}$ Includes cogenerators.

${ }^{3}$ Excludes uses for lease and plant fuel.

Includes Federal and State taxes on diesel fuel and excludes county and local taxes.

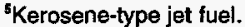

'Sales weighted average price for all grades. Includes Federal and State taxes and excludes county and local taxes.

${ }^{7}$ Compressed natural gas used as a vehicle fuel. Price includes estimated motor vehicle fuel taxes.

Includes all electric power generators except cogenerators, which produce electricity as a by-product of other processes.

'Weighted averages of end-use fuel prices are derived from the prices shown in each sector and the corresponding sectoral consumption.

Btu = British thermal unit.

Note: 1993 and 1994 figures may differ from published data due to internal rounding.

Sources: 1993 prices for gasoline, distilate, and jet fuel are based on prices in the Energy Information Administration (EIA), Petroleum Marketing Annual 1993, DOE/EIA-0487(93) Washington, DC, July 1994). 1994 prices for gasoline, distillate, and jel fuel are based on prices in various 1994 issues of EIA, Petroleum Marketing Monthly, DOE/EIA-0380(94/1-12) (Washington, DC, 1994). 1993 and 1994 prices for all other petroleum products are derived from the EIA, State Energy Price and Expenditure Report 1991, DOE/EIA-0376(91) Washington, DC, September 1993). 1993 residential, commercial, and transportation natural gas delivered prices: EIA, Natural Gas Annual 1993, DOE/EIA-0131(93) (Washington, DC, October 1994). 1993 electric generators natural gas delivered prices: Form FERC-423, "Monthly Report of Cost and Quality of Fuels for Electric Plants." 1993 and 1994 industrial gas delivered prices are based on ElA, Manufacturing Energy Consumption Survey 1991. 1994 residential and commercial natural gas delivered prices: EIA, Natural Gas Monthly, DOE/EIA-0130(95/6) (Washington, DC, June 1995). Other 1994 natural gas delivered prices: EIA, AEO96 National Energy Modeling System runs TECH01.D031396A (Slow Technology), AEO96B.D101995C (reference), and TECH04.D031396B (Rapid Technology). Values for 1993 coal prices have been estimated from EIA, State Price and Expenditure Report 1992 DOE/EIA-0376(92) (Washington, DC, December 1994) using consumption quantities aggregated from ElA, State Energy Data Report 1992, Consumption Estimates, DOE/EIA-0214(92) (Washington, DC, May 1994). Values for 1994 coal prices have been estimated from EIA, State Price and Expenditure Report 1993, DOE/EIA-0376(93) (Washington, DC, December 1995) using consumption quantities aggregated from EIA, State Energy Data Report 1993, Consumption Estimates, DOE/EIA-0214(93) (Washington, DC, May 1995). 1993 residential electricity prices derived from ElA, Short Term Energy Outlook, DOE/EIA-0202(95/3Q) (Washington, DC, August 1995). 1993 and 1994 electricity prices for commercial, industrial, and transportation: ElA AEO96 National Energy Modeling System run TECH01.D031396A (Slow Technology), AEO96B.D101995C (reference), and TECH04.D031396B (Rapid Technology). Projections: EIA, AEO96 National Energy Modeling System runs TECH01.D031396A (Slow Technology), AEO96B.D101995C (reference), and TECHO4.D031396B (Rapid Technology). 
Table B4. Residential Sector Key Indicators and End-Use Consumption (Quadrillion Btu per Year, Unless Otherwise Noted)

\begin{tabular}{c|c|c|c|c|c|c|}
\hline \multirow{2}{*}{ Key Indicators and Consumption } & & & \multicolumn{2}{|c}{ Projections } \\
\cline { 3 - 5 } & & & & \multicolumn{2}{|c|}{2000} \\
\cline { 3 - 5 } & & & & Slow & Rapid \\
Technology & Reference & Technology \\
\hline
\end{tabular}

Household Characteristics

Households (millions)

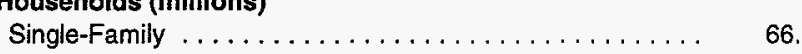

Multifamily ........................... 24.25

Mobile Homes . . . . . . . . . . . . . . . . . 5.57

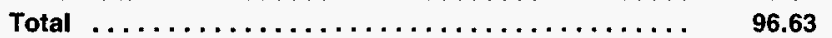

Housing Starts (millions)

Single-Family . . . . . . . . . . . . . . . . . . .

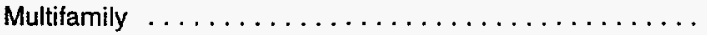

Mobile Homes . . . . . . . . . . . . . . . . . . . . . .

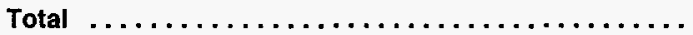

Average House Square Footage

0.16

0.26

1.55

1630

67.80

24.40

5.71

97.92

68.60
24.56
5.84
99.00

1.19

0.25

0.31

1.75

1637

1.00

0.26

0.30

1.56

1643

Energy Intensity

Million Btu Consumed per Household

Delivered Energy Consumption .................

Electricity Related Losses . . . . . . . . . . . . . . .

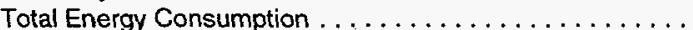

Thousand Btu Consumed per Square Foot

Delivered Energy Consumption . . . . . . . . . . . . .

Electricity Related Losses . . . . . . . . . . . . . . . . . .

Total Energy Consumption .

.......................

Delivered Energy Consumption by Fuel

\section{Distillate}

108.76

78.29

187.06

66.71

48.02

114.74
106.34

77.91

184.26

64.95

47.59

112.54
105.05

78.08

183.13

63.95

47.53

111.48

72.56
25.58
6.14
104.29

72.57
25.58
6.14
104.29

72.58

25.59

6.14

104.31

1.03
0.35
0.23
1.61
1666

1.03

0.36

0.23

1.62

1666

105.06
76.94
182.00

103.94

76.34

180.28

101.17

73.22

174.39

62.40

45.83

108.24

60.74

43.96

104.70

Space Heating $\ldots \ldots \ldots \ldots \ldots \ldots \ldots \ldots \ldots \ldots \ldots$

Water Heating . . . . . . . . . . . . . . . . . .

Other Uses ${ }^{1} \ldots \ldots \ldots \ldots \ldots \ldots \ldots \ldots \ldots \ldots \ldots$

Delivered Energy $\ldots \ldots \ldots \ldots \ldots \ldots \ldots \ldots \ldots \ldots$

Liquefied Petroleum Gas

Space Heating $\ldots \ldots \ldots \ldots \ldots \ldots \ldots \ldots \ldots \ldots$

Water Heating..$\ldots \ldots \ldots \ldots \ldots \ldots \ldots \ldots \ldots$

Cooking ${ }^{2} \ldots \ldots \ldots \ldots \ldots \ldots \ldots \ldots \ldots \ldots \ldots \ldots$

Other Uses ${ }^{3} \ldots \ldots \ldots \ldots \ldots \ldots \ldots \ldots \ldots \ldots \ldots$

Delivered Energy

Delivered Energy

\section{Electricity}

Space Heating

Space Cooling . . . . . . . . . . . . . . . . . . . .

Water Heating . . . . . . . . . . . . . . . . . . . .

Refrigeration $\ldots \ldots \ldots \ldots \ldots \ldots \ldots \ldots \ldots \ldots \ldots \ldots$

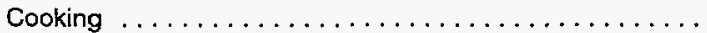

Clothes Dryers $\ldots \ldots \ldots \ldots \ldots \ldots \ldots \ldots \ldots \ldots$

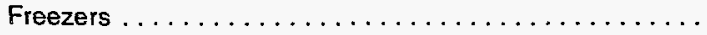

Lighting . . . . . . . . . . . . . . . . . . .

Other Uses ${ }^{4}$

Delivered Energy

$\begin{array}{lll}0.42 & 0.41 & 0.40 \\ 0.48 & 0.48 & 0.52 \\ 0.35 & 0.36 & 0.36 \\ 0.47 & 0.46 & 0.44 \\ 0.10 & 0.10 & 0.10 \\ 0.17 & 0.17 & 0.17 \\ 0.14 & 0.14 & 0.13 \\ 0.31 & 0.31 & 0.32 \\ 0.95 & 1.01 & 1.05 \\ \mathbf{3 . 3 9} & \mathbf{3 . 4 3} & \mathbf{3 . 4 9}\end{array}$

$\begin{array}{ll}0.44 & 0.42 \\ 0.50 & 0.48 \\ 0.37 & 0.30 \\ 0.39 & 0.38 \\ 0.10 & 0.10 \\ 0.18 & 0.18 \\ 0.10 & 0.10 \\ 0.33 & 0.31 \\ 1.29 & 1.29 \\ 3.70 & 3.57\end{array}$


Table B4. Residential Sector Key Indicators and End-Use Consumption (Continued) (Quadrillion Btu per Year, Unless Otherwise Noted)

\begin{tabular}{c|c|c|c|c|c|c|c|c|c|c|}
\hline \multicolumn{9}{c|}{ Projections } & \multicolumn{2}{c}{$\begin{array}{c}\text { Annual Growth } \\
1994-2015 \text { (percent) }\end{array}$} \\
\hline $\begin{array}{c}\text { Slow } \\
\text { Technology }\end{array}$ & Reference & $\begin{array}{c}\text { Rapid } \\
\text { Technology }\end{array}$ & $\begin{array}{c}\text { Slow } \\
\text { Technology }\end{array}$ & Reference & $\begin{array}{c}\text { Rapid } \\
\text { Technology }\end{array}$ & $\begin{array}{c}\text { Slow } \\
\text { Technology }\end{array}$ & Reference & $\begin{array}{c}\text { Rapid } \\
\text { Technology }\end{array}$ & $\begin{array}{c}\text { Slow } \\
\text { Technology }\end{array}$ & $\begin{array}{c}\text { Rapid } \\
\text { Reference }\end{array}$ \\
\hline
\end{tabular}

\begin{tabular}{|c|c|c|c|c|c|c|c|c|c|c|c|}
\hline 76.79 & 76.80 & 76.83 & 80.97 & 81.00 & 81.06 & 85.19 & 85.27 & 85.35 & 1.1 & 1.1 & 1.1 \\
\hline 27.03 & 27.05 & 27.07 & 28.74 & 28.77 & 28.82 & 30.60 & 30.67 & 30.74 & 1.1 & 1.1 & 1.1 \\
\hline 6.36 & 6.37 & 6.37 & 6.58 & 6.58 & 6.58 & 6.80 & 6.80 & 6.81 & 0.8 & 0.8 & 0.8 \\
\hline 110.18 & 110.21 & 110.26 & 116.29 & 116.36 & 116.46 & 122.59 & 122.73 & 122.90 & 1.1 & 1.1 & 1.1 \\
\hline 1.09 & 1.09 & 1.10 & 1.05 & 1.06 & 1.06 & 1.09 & 1.10 & 1.10 & N/A & N/A & N/A \\
\hline 0.42 & 0.42 & 0.42 & 0.47 & 0.48 & 0.48 & 0.48 & 0.49 & 0.50 & N/A & $N / A$ & $\mathrm{~N} /$ \\
\hline 0.24 & 0.24 & 0.24 & 0.24 & 0.24 & 0.24 & 0.25 & 0.25 & 0.25 & N/A & N/A & N/A \\
\hline 1.74 & 1.75 & 1.76 & 1.76 & 1.78 & 1.78 & 1.82 & 1.84 & 1.85 & N/A & N/A & N/A \\
\hline 1684 & 1684 & 1684 & 1698 & 1698 & 1698 & 1710 & 1710 & 1710 & 0.2 & 0.2 & 0.2 \\
\hline
\end{tabular}

\begin{tabular}{|c|c|c|c|c|c|c|c|c|c|c|c|}
\hline 104.06 & 101.65 & 96.93 & 104.45 & 100.64 & 93.76 & 105.50 & 100.05 & 91.75 & 0.0 & -0.3 & -0.7 \\
\hline 78.80 & 77.33 & 72.00 & 80.60 & 77.98 & 72.07 & 80.50 & 76.62 & 72.28 & 0.2 & -0.1 & -0.3 \\
\hline 182.86 & 178.98 & 168.93 & 185.06 & 178.62 & 165.83 & 186.00 & 176.67 & 164.03 & 0.0 & -0.2 & -0.5 \\
\hline 61.79 & 60.36 & 57.55 & 61.50 & 59.26 & 55.21 & 61.68 & 58.49 & 53.64 & -0.2 & -0.5 & -0.9 \\
\hline 46.79 & 45.92 & 42.75 & 47.46 & 45.91 & 42.44 & 47.06 & 44.79 & 42.26 & -0.1 & -0.3 & -0.6 \\
\hline 108.58 & 106.28 & 100.31 & 108.96 & 105.17 & 97.64 & 108.74 & 103.28 & 95.90 & -0.2 & -0.4 & -0.7 \\
\hline
\end{tabular}

\begin{tabular}{|c|c|c|c|c|c|c|c|c|c|c|c|}
\hline 0.75 & 0.72 & 0.70 & 0.74 & 0.70 & 0.68 & 0.76 & 0.70 & 0.65 & -0.1 & -0.5 & -0.8 \\
\hline 0.10 & 0.09 & 0.09 & 0.10 & 0.09 & 0.09 & 0.10 & 0.09 & 0.09 & 0.0 & -0.3 & -0.4 \\
\hline 0.00 & 0.00 & 0.00 & 0.00 & 0.00 & 0.00 & 0.00 & 0.00 & 0.00 & 1.4 & 1.4 & 1.4 \\
\hline 0.85 & 0.82 & 0.80 & 0.84 & 0.80 & 0.77 & 0.86 & 0.80 & 0.74 & -0.1 & -0.4 & -0.7 \\
\hline 0.23 & 0.23 & 0.22 & 0.21 & 0.21 & 0.20 & 0.20 & 0.19 & 0.18 & -1.4 & -1.6 & -1.9 \\
\hline 0.05 & 0.04 & 0.04 & 0.05 & 0.04 & 0.04 & 0.05 & 0.04 & 0.04 & -0.6 & -1.0 & -1.1 \\
\hline 0.02 & 0.02 & 0.02 & 0.02 & 0.02 & 0.02 & 0.02 & 0.02 & 0.02 & -0.8 & -0.8 & -0.8 \\
\hline 0.07 & 0.07 & 0.07 & 0.07 & 0.07 & 0.07 & 0.08 & 0.08 & 0.08 & 1.2 & 1.2 & 1.2 \\
\hline 0.37 & 0.36 & 0.36 & 0.35 & 0.34 & 0.33 & 0.35 & 0.33 & 0.32 & -0.8 & -1.0 & -1.1 \\
\hline 3.77 & 3.70 & 3.52 & 3.91 & 3.82 & 3.61 & 4.01 & 3.88 & 3.65 & 0.7 & 0.6 & 0.3 \\
\hline 0.00 & 0.00 & 0.00 & 0.00 & 0.00 & 0.00 & 0.01 & 0.00 & 0.00 & 14.6 & 14.2 & 13.9 \\
\hline 1.37 & 1.32 & 1.29 & 1.44 & 1.35 & 1.23 & 1.51 & 1.39 & 1.21 & 0.8 & 0.4 & -0.3 \\
\hline 0.20 & 0.20 & 0.20 & 0.22 & 0.20 & 0.20 & 0.23 & 0.21 & 0.21 & 1.3 & 0.9 & 0.9 \\
\hline 0.05 & 0.05 & 0.05 & 0.05 & 0.05 & 0.05 & 0.05 & 0.05 & 0.05 & 0.2 & 0.2 & 0.1 \\
\hline 0.06 & 0.06 & 0.06 & 0.06 & 0.06 & 0.06 & 0.07 & 0.07 & 0.07 & 1.0 & 1.0 & 1.0 \\
\hline 5.46 & 5.33 & 5.12 & 5.68 & 5.50 & 5.16 & 5.88 & 5.61 & 5.20 & 0.8 & 0.5 & 0.2 \\
\hline 0.48 & 0.46 & 0.43 & 0.51 & 0.48 & 0.43 & 0.54 & 0.49 & 0.44 & 1.4 & 0.9 & 0.4 \\
\hline 0.54 & 0.52 & 0.47 & 0.59 & 0.53 & 0.48 & 0.63 & 0.54 & 0.49 & 1.3 & 0.5 & 0.1 \\
\hline 0.38 & 0.37 & 0.26 & 0.39 & 0.39 & 0.23 & 0.41 & 0.40 & 0.22 & 0.6 & 0.5 & -2.2 \\
\hline 0.36 & 0.35 & 0.34 & 0.35 & 0.32 & 0.31 & 0.35 & 0.30 & 0.30 & -1.3 & -2.0 & -2.1 \\
\hline 0.11 & 0.11 & 0.11 & 0.12 & 0.11 & 0.11 & 0.12 & 0.12 & 0.12 & 1.0 & 0.9 & 0.9 \\
\hline 0.19 & 0.19 & 0.19 & 0.20 & 0.20 & 0.19 & 0.21 & 0.21 & 0.20 & 0.9 & 0.9 & 0.8 \\
\hline 0.08 & 0.08 & 0.08 & 0.07 & 0.07 & 0.07 & 0.07 & 0.07 & 0.07 & -3.1 & -3.1 & -3.3 \\
\hline 0.36 & 0.34 & 0.30 & 0.39 & 0.35 & 0.26 & 0.41 & 0.36 & 0.20 & 1.3 & 0.7 & -2.0 \\
\hline 1.57 & 1.57 & 1.57 & 1.93 & 1.93 & 1.93 & 2.35 & 2.36 & 2.36 & 4.1 & 4.1 & 4.1 \\
\hline 4.07 & 4.00 & 3.76 & 4.53 & 4.37 & 4.02 & 5.09 & 4.84 & 4.39 & 1.9 & 1.7 & .2 \\
\hline
\end{tabular}


Table B4. Residential Sector Key Indicators and End-Use Consumption (Continued) (Quadrillion Btu per Year, Unless Otherwise Noted)

\begin{tabular}{|c|c|c|c|c|c|c|}
\hline \multirow[b]{3}{*}{ Key Indicators and Consumption } & \multirow[b]{3}{*}{1993} & \multirow[b]{3}{*}{1994} & \multirow[b]{3}{*}{1995} & \multicolumn{3}{|c|}{ Projections } \\
\hline & & & & \multicolumn{3}{|c|}{2000} \\
\hline & & & & $\begin{array}{c}\text { Slow } \\
\text { Technology }\end{array}$ & Reference & $\begin{array}{c}\text { Rapid } \\
\text { Technology }\end{array}$ \\
\hline \multicolumn{7}{|l|}{ Marketed Renewables } \\
\hline 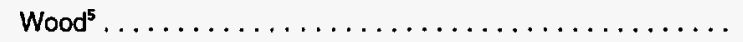 & 0.57 & 0.55 & 0.55 & 0.58 & 0.57 & 0.56 \\
\hline Delivered Energy $\ldots \ldots \ldots \ldots \ldots \ldots \ldots \ldots \ldots \ldots$ & 0.57 & 0.55 & 0.55 & 0.58 & 0.57 & 0.56 \\
\hline Other Fuels $^{6} \ldots \ldots \ldots \ldots \ldots \ldots \ldots \ldots \ldots \ldots \ldots$ & 0.13 & 0.12 & 0.12 & 0.12 & 0.12 & 0.12 \\
\hline \multicolumn{7}{|l|}{ Delivered Energy Consumption by End-Use } \\
\hline Space Heating $\ldots \ldots \ldots \ldots \ldots \ldots \ldots \ldots \ldots \ldots$ & 5.76 & 5.58 & 5.49 & 5.82 & 5.74 & 5.58 \\
\hline 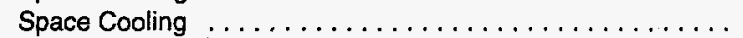 & 0.48 & 0.48 & 0.52 & 0.51 & 0.51 & 0.48 \\
\hline Water Heating $\ldots \ldots \ldots \ldots \ldots \ldots \ldots \ldots$ & 1.77 & 1.79 & 1.80 & 1.83 & 1.82 & 1.74 \\
\hline Refrigeration $\ldots \ldots \ldots \ldots \ldots \ldots \ldots \ldots \ldots$ & 0.47 & 0.46 & 0.44 & 0.39 & 0.39 & 0.38 \\
\hline 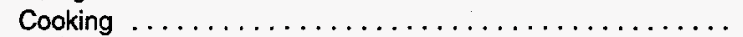 & 0.29 & 0.30 & 0.30 & 0.32 & 0.32 & 0.32 \\
\hline Clothes Dryers . . . . . . . . . . . . . . . . . . & 0.22 & 0.23 & 0.23 & 0.23 & 0.23 & 0.23 \\
\hline Freezers $\ldots \ldots \ldots \ldots \ldots \ldots \ldots \ldots \ldots \ldots \ldots$ & 0.14 & 0.14 & 0.13 & 0.10 & 0.10 & 0.10 \\
\hline Lighting $\ldots \ldots \ldots \ldots \ldots \ldots \ldots \ldots \ldots$ & 0.31 & 0.31 & 0.32 & 0.34 & 0.33 & 0.31 \\
\hline 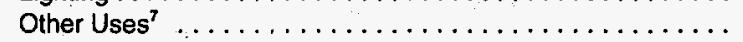 & 1.07 & 1.13 & 1.17 & 1.41 & 1.41 & 1.41 \\
\hline Delivered Energy $\ldots \ldots \ldots \ldots \ldots \ldots \ldots \ldots \ldots$ & 10.51 & 10.41 & 10.40 & 10.96 & 10.84 & 10.55 \\
\hline \multicolumn{7}{|l|}{ Electricity Related Losses by End-Use } \\
\hline 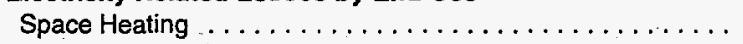 & 0.95 & 0.90 & 0.89 & 0.97 & 0.95 & 0.91 \\
\hline 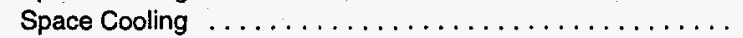 & 1.07 & 1.08 & 1.16 & 1.10 & 1.08 & 1.02 \\
\hline Water Heating $\ldots \ldots \ldots \ldots \ldots \ldots \ldots \ldots$ & 0.78 & 0.79 & 0.79 & 0.79 & 0.79 & 0.65 \\
\hline Refrigeration $\ldots \ldots \ldots \ldots \ldots \ldots \ldots \ldots \ldots$ & 1.05 & 1.02 & 0.98 & 0.84 & 0.83 & 0.82 \\
\hline Cooking $\ldots \ldots \ldots \ldots \ldots \ldots \ldots \ldots \ldots \ldots \ldots \ldots \ldots \ldots$ & 0.21 & 0.22 & 0.22 & 0.22 & 0.22 & 0.22 \\
\hline Clothes Dryers $\ldots \ldots \ldots \ldots \ldots \ldots \ldots \ldots \ldots \ldots \ldots \ldots$ & 0.38 & 0.39 & 0.39 & 0.39 & 0.39 & 0.39 \\
\hline Freezers $\ldots \ldots \ldots \ldots \ldots \ldots \ldots \ldots \ldots \ldots \ldots \ldots \ldots$ & 0.31 & 0.30 & 0.28 & 0.21 & 0.21 & 0.20 \\
\hline Lighting $\ldots \ldots \ldots \ldots \ldots \ldots \ldots \ldots \ldots$ & 0.70 & 0.70 & 0.70 & 0.73 & 0.71 & 0.67 \\
\hline Other Uses ${ }^{7} \ldots \ldots \ldots \ldots \ldots \ldots \ldots \ldots \ldots \ldots \ldots$ & 2.13 & 2.24 & 2.32 & 2.77 & 2.77 & 2.75 \\
\hline Total Electricity Related Losses . . . . . . . . . . . . . . & 7.57 & 7.63 & 7.73 & 8.02 & 7.96 & 7.64 \\
\hline \multicolumn{7}{|l|}{ Total Energy Consumption by End-Use } \\
\hline Space Heating $\ldots \ldots \ldots \ldots \ldots \ldots \ldots \ldots \ldots \ldots$ & 6.70 & 6.48 & 6.38 & 6.78 & 6.69 & 6.49 \\
\hline 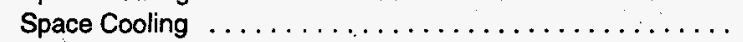 & 1.54 & 1.56 & 1.68 & 1.61 & 1.59 & 1.50 \\
\hline Water Heating $\ldots \ldots \ldots \ldots \ldots \ldots \ldots \ldots \ldots$ & 2.55 & 2.58 & 2.58 & 2.62 & 2.60 & 2.39 \\
\hline Refrigeration $\ldots \ldots \ldots \ldots \ldots \ldots \ldots \ldots \ldots \ldots \ldots$ & 1.52 & 1.48 & 1.43 & 1.23 & 1.22 & 1.21 \\
\hline$\ldots \ldots \ldots \ldots \ldots \ldots \ldots \ldots$ & 0.51 & 0.52 & 0.52 & 0.54 & 0.54 & 0.54 \\
\hline Clothes Dryers $\ldots \ldots \ldots \ldots \ldots \ldots \ldots \ldots \ldots$ & 0.60 & 0.61 & 0.61 & 0.63 & 0.63 & 0.62 \\
\hline Freezers $\ldots \ldots \ldots \ldots \ldots \ldots \ldots \ldots \ldots \ldots \ldots \ldots$ & 0.46 & 0.44 & 0.41 & 0.30 & 0.30 & 0.30 \\
\hline$\ldots \ldots \ldots \ldots \ldots$ & 1.01 & 1.01 & 1.02 & 1.08 & 1.04 & 0.99 \\
\hline$\ldots \ldots \ldots \ldots \ldots \ldots \ldots \ldots \ldots$ & 3.19 & 3.36 & 3.49 & 4.18 & 4.18 & 4.17 \\
\hline Total $\ldots \ldots \ldots \ldots \ldots \ldots \ldots \ldots \ldots \ldots \ldots$ & 18.08 & 18.04 & 18.13 & 18.98 & 18.80 & 18.19 \\
\hline \multicolumn{7}{|l|}{ Non-Marketed Renewables } \\
\hline Geothermal ${ }^{8}$. & 0.01 & 0.01 & 0.01 & 0.01 & 0.01 & 0.02 \\
\hline$\ldots \ldots \ldots \ldots \ldots \ldots \ldots \ldots$ & 0.01 & 0.01 & 0.01 & 0.01 & 0.01 & 0.01 \\
\hline Total $\ldots \ldots \ldots \ldots \ldots \ldots \ldots \ldots \ldots \ldots \ldots \ldots$ & 0.02 & 0.02 & 0.02 & 0.02 & 0.02 & 0.02 \\
\hline
\end{tabular}

Includes such appliances as swimming-pool and hot-tub heaters.

${ }^{2}$ Does not include outdoor grills.

${ }^{3}$ Includes such appliances as swimming-pool heaters, outdoor grills, and outdoor lighting (natural gas).

4 includes such appliances as microwave ovens, television sets, and dishwashers.

Includes wood used for primary and secondary heating in wood stoves or fireplaces as reported by the Residential Energy Consumption Survey 1993.

Includes kerosene and coal.

TIncludes such appliances as swimming-pool heaters, hot-tub heaters, outdoor grills, outdoor lighting (natural gas), microwave ovens, television sets, and dishwashers.

Includes primary energy displaced by ground-source heat pumps in space heating and cooling applications.

Includes primary energy displaced by solar thermal water heaters. 
Table B4. Residential Sector Key Indicators and End-Use Consumption (Continued) (Quadrillion Btu per Year, Unless Otherwise Noted)

\begin{tabular}{|c|c|c|c|c|c|c|c|c|c|c|c|}
\hline \multicolumn{9}{|c|}{ Projections } & \multirow{2}{*}{\multicolumn{3}{|c|}{$\begin{array}{c}\text { Annual Growth } \\
\text { 1994-2015 (percent) }\end{array}$}} \\
\hline \multicolumn{3}{|c|}{2005} & \multicolumn{3}{|c|}{2010} & \multicolumn{3}{|c|}{2015} & & & \\
\hline $\begin{array}{c}\text { Slow } \\
\text { Technology }\end{array}$ & Reference & \begin{tabular}{|c|} 
Rapid \\
Technology
\end{tabular} & $\begin{array}{c}\text { Slow } \\
\text { Technology }\end{array}$ & Reference & $\begin{array}{c}\text { Papid } \\
\text { Technology }\end{array}$ & $\begin{array}{c}\text { Slow } \\
\text { Technology }\end{array}$ & Reference & $\begin{array}{c}\text { Rapid } \\
\text { Technology }\end{array}$ & $\begin{array}{c}\text { Slow } \\
\text { Technology }\end{array}$ & Reference & $\begin{array}{c}\text { Rapid } \\
\text { Technology }\end{array}$ \\
\hline 0.60 & 0.58 & 0.55 & 0.62 & 0.59 & 0.54 & 0.64 & 0.59 & 0.53 & 0.7 & 0.3 & -0.2 \\
\hline 0.60 & 0.58 & 0.55 & 0.62 & 0.59 & 0.54 & 0.64 & 0.59 & 0.53 & 0.7 & 0.3 & 0.2 \\
\hline 0.12 & 0.11 & 0.11 & 0.12 & 0.11 & 0.10 & 0.11 & 0.10 & 0.09 & -0.5 & -0.8 & -1.3 \\
\hline 5.95 & 5.80 & 5.53 & 6.11 & 5.90 & 5.55 & 6.26 & 5.96 & 5.54 & 0.6 & 0.3 & 0.0 \\
\hline 0.54 & 0.52 & 0.48 & 0.59 & 0.53 & 0.48 & 0.63 & 0.54 & 0.49 & 1.3 & 0.5 & 0.1 \\
\hline 1.89 & 1.83 & 1.68 & 1.97 & 1,87 & 1.59 & 2.06 & 1.93 & 1.56 & 0.7 & 0.3 & -0.6 \\
\hline 0.36 & 0.35 & 0.34 & 0.35 & 0.32 & 0.31 & 0.35 & 0.30 & 0.30 & -1.3 & -2.0 & -2.1 \\
\hline 0.34 & 0.33 & 0.33 & 0.35 & 0.34 & 0.34 & 0.37 & 0.35 & 0.35 & 1.0 & 0.7 & 0.8 \\
\hline 0.24 & 0.24 & 0.24 & 0.25 & 0.25 & 0.25 & 0.26 & 0.26 & 0.26 & 0.8 & 0.8 & 0.7 \\
\hline 0.08 & 0.08 & 0.08 & 0.07 & 0.07 & 0.07 & 0.07 & 0.07 & 0.07 & -3.1 & -3.1 & -3.3 \\
\hline 0.36 & 0.34 & 0.30 & 0.39 & 0.35 & 0.26 & 0.41 & 0.36 & 0.20 & 1.3 & 0.7 & -2.0 \\
\hline 1.71 & 1.71 & 1.71 & 2.07 & 2.07 & 2.07 & 2.51 & 2.51 & 2.51 & 3.9 & 3.9 & 3.9 \\
\hline 11.47 & 11.20 & 10.69 & 12.15 & 11.71 & 10.92 & 12.93 & 12.28 & 11.28 & 1.0 & 0.8 & 0.4 \\
\hline 1.01 & 0.98 & 0.90 & 1.05 & 0.99 & 0.91 & 1.04 & 0.95 & 0.88 & 0.7 & 0.3 & -0.1 \\
\hline 1.16 & 1.11 & 1.00 & 1.21 & 1.10 & 1.00 & 1.22 & 1.04 & 0.98 & 0.6 & -0.1 & -0.4 \\
\hline 0.81 & 0.80 & 0.55 & 0.81 & 0.80 & 0.48 & 0.79 & 0.77 & 0.44 & 0.0 & -0.1 & -2.6 \\
\hline 0.76 & 0.74 & 0.73 & 0.71 & 0.65 & 0.65 & 0.68 & 0.58 & 0.60 & -1.9 & -2.6 & -2.5 \\
\hline 0.23 & 0.23 & 0.23 & 0.24 & 0.23 & 0.24 & 0.23 & 0.23 & 0.24 & 0.3 & 0.2 & 0.4 \\
\hline 0.41 & 0.40 & 0.39 & 0.41 & 0.41 & 0.41 & 0.41 & 0.41 & 0.41 & 0.3 & 0.3 & 0.3 \\
\hline 0.17 & 0.17 & 0.17 & 0.15 & 0.15 & 0.14 & 0.14 & 0.13 & 0.13 & -3.7 & -3.7 & -3.8 \\
\hline 0.78 & 0.73 & 0.64 & 0.80 & 0.73 & 0.54 & 0.80 & 0.71 & 0.41 & 0.6 & 0.0 & -2.5 \\
\hline 3.36 & 3.35 & 3.32 & 3.98 & 3.99 & 4.03 & 4.56 & 4.58 & 4.78 & 3.5 & 3.5 & 3.7 \\
\hline 8.68 & 8.52 & 7.94 & 9.37 & 9.07 & 8.39 & 9.87 & 9.40 & 8.88 & 1.2 & 1.0 & 0.7 \\
\hline 6.96 & 6.78 & 6.43 & 7.16 & 6.90 & 6.46 & 7.31 & 6.91 & 6.42 & 0.6 & 0.3 & 0.0 \\
\hline 1.70 & 1.63 & 1.48 & 1.80 & 1.63 & 1.49 & 1.85 & 1.58 & 1.47 & 0.8 & 0.1 & -0.2 \\
\hline 2.69 & 2.63 & 2.23 & 2.78 & 2.67 & 2.07 & 2.85 & 2.70 & 2.00 & 0.5 & 0.2 & -1.2 \\
\hline 1.12 & 1.09 & 1.07 & 1.06 & 0.97 & 0.96 & 1.03 & 0.89 & 0.90 & -1.7 & -2.4 & -2.4 \\
\hline 0.57 & 0.56 & 0.56 & 0.59 & 0.57 & 0.58 & 0.61 & 0.58 & 0.59 & 0.8 & 0.5 & 0.6 \\
\hline 0.65 & 0.65 & 0.63 & 0.67 & 0.67 & 0.65 & 0.67 & 0.67 & 0.67 & 0.5 & 0.5 & 0.5 \\
\hline 0.25 & 0.25 & 0.24 & 0.22 & 0.22 & 0.21 & 0.21 & 0.20 & 0.20 & -3.5 & -3.5 & -3.6 \\
\hline 1.14 & 1.08 & 0.95 & 1.19 & 1.09 & 0.80 & 1.21 & 1.07 & 0.62 & 0.9 & 0.3 & -2.3 \\
\hline 5.06 & 5.06 & 5.03 & 6.05 & 6.06 & 6.10 & 7.07 & 7.09 & 7.29 & 3.6 & 3.6 & 3.7 \\
\hline 20.15 & 19.73 & 18.63 & 21.52 & 20.78 & 19.31 & 22.80 & 21.68 & 20.16 & 1.1 & 0.9 & 0.5 \\
\hline 0.02 & 0.02 & 0.02 & 0.02 & 0.02 & 0.02 & 0.02 & 0.03 & 0.03 & 4.1 & 4.8 & 4.8 \\
\hline 0.01 & 0.01 & 0.01 & 0.01 & 0.01 & 0.01 & 0.01 & 0.01 & 0.01 & -0.3 & -0.3 & -0.3 \\
\hline 0.02 & 0.02 & 0.03 & 0.03 & 0.03 & 0.03 & 0.03 & 0.03 & 0.03 & 2.9 & 3.5 & 3.5 \\
\hline
\end{tabular}

Btu $=$ British thermal unit.

N/A = Not applicable.

Note: Totals may not equal sum of components due to independent rounding.

Sources: 1993: Energy information Administration (EIA), State Energy Data Report, DOEJEIA-0214(93) (Washington, DC, July 1995). 1994: EIA, Short-Term Energy Outlook,

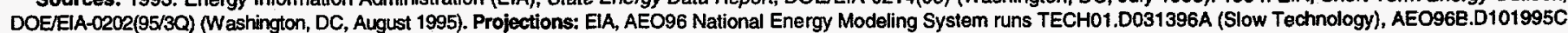
(reference), and TECH04.D031396B (Rapid Technology). 
Table B5. Commercial Sector Key Indicators and End-Use Consumption (Quadrillion Btu per Year, Unless Otherwise Noted)

\begin{tabular}{|c|c|c|c|c|c|c|}
\hline \multirow[b]{3}{*}{ Key Indicators and Consumption } & \multirow[b]{3}{*}{1993} & \multirow[b]{3}{*}{1994} & \multirow[b]{3}{*}{1995} & \multicolumn{3}{|c|}{ Projections } \\
\hline & & & & \multicolumn{3}{|c|}{2000} \\
\hline & & & & $\begin{array}{c}\text { Slow } \\
\text { Technology }\end{array}$ & Reference & $\begin{array}{c}\text { Rapid } \\
\text { Technology }\end{array}$ \\
\hline
\end{tabular}

Key Indicators

Total Floor Space (billion square feet)

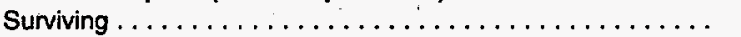

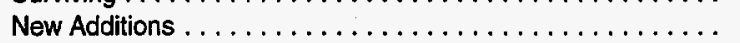

Total $\ldots \ldots \ldots \ldots \ldots \ldots \ldots \ldots \ldots \ldots \ldots \ldots \ldots \ldots$

$67.2 \quad 68.2 \quad 69.2$

$\begin{array}{lll}1.8 & 1.8 & 1.9\end{array}$

$69.1 \quad 70.0 \quad 71.0$

Energy Consumption Intensity

(thousand Btu per square foot)

Delivered Energy Consumption $\ldots \ldots \ldots \ldots \ldots \ldots \ldots$

Electricity Related Losses . . . . . . . . . . . . . . . .

Total Energy Consumption

$98.9 \quad 99.3$

$97.3 \quad 99.3$

$196.2 \quad 198.6$

99.4

99.8

199.2

98.4
96.4

194.8

73.7
1.7

75.4

73.7

1.7

75.4

1.7

75.4

Delivered Energy Consumption by Fuel

\section{Electricity}

Space Heating $\ldots \ldots \ldots \ldots \ldots \ldots \ldots \ldots \ldots \ldots \ldots$

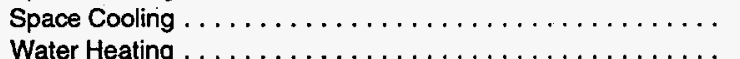

Water Heating $\ldots \ldots \ldots \ldots \ldots \ldots \ldots \ldots \ldots \ldots \ldots \ldots \ldots \ldots$
Ventilation $\ldots \ldots \ldots \ldots \ldots \ldots \ldots \ldots \ldots$

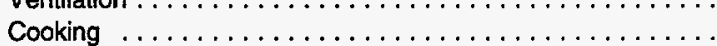

Lighting $\ldots \ldots \ldots \ldots \ldots \ldots \ldots \ldots \ldots \ldots \ldots \ldots \ldots$

Refrigeration .

Office Equipment (PC) $\ldots \ldots \ldots \ldots \ldots \ldots \ldots \ldots \ldots$

Office Equipment (non-PC) $\ldots \ldots \ldots \ldots \ldots \ldots \ldots \ldots$

Other Uses ${ }^{t} \ldots \ldots \ldots \ldots \ldots \ldots \ldots \ldots \ldots \ldots \ldots$

Delivered Energy

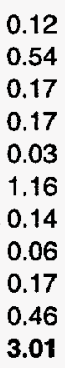

0.11

0.53

0.11

0.54

0.17

0.17

0.03

1.15

0.14

0.07

0.18

0.57

3.13

0.56

0.17

0.17

0.03

1.16

0.14

0.07

0.18

0.59

3.20

Natural Gas ${ }^{2}$

Space Heating

Space Cooling..$\ldots \ldots \ldots \ldots \ldots \ldots \ldots \ldots \ldots \ldots \ldots$

Water Heating . . . . . . . . . . . . . . . . . . . . . .

Cooking

Other Uses ${ }^{3}$

Delivered Energy

Distillate

Space Heating $\ldots \ldots \ldots \ldots \ldots \ldots \ldots \ldots \ldots \ldots \ldots, 0.20$

Water Heating $\ldots \ldots \ldots \ldots \ldots \ldots \ldots \ldots \ldots \ldots \ldots \ldots$

Other Uses 4

Delivered Energy . . . . . . . . . . . . . . . . . . . . .

0.06

0.06

0.46

0.20

0.06

0.17

0.42

0.05

0.17

Other Fuels $\mathbf{s}^{5}$

0.37

0.39

0.40

Marketed Renewable Fuels

Biomass

Delivered Energy

0.00

0.00

0.00

0.00

0.00

0.00

1.66

0.56

Space Heating $\ldots \ldots \ldots \ldots \ldots \ldots \ldots \ldots \ldots \ldots$

Space Cooling . . . . . . . . . . . . . . . . . . . . .

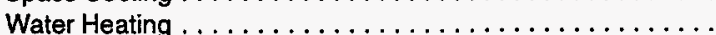

Ventilation..$\ldots \ldots \ldots \ldots \ldots \ldots \ldots \ldots \ldots \ldots \ldots \ldots$

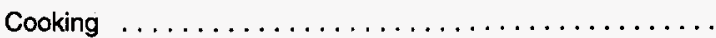

Lighting $\ldots \ldots \ldots \ldots \ldots \ldots \ldots \ldots \ldots \ldots \ldots \ldots \ldots$

Refrigeration $\ldots \ldots \ldots \ldots \ldots \ldots \ldots \ldots \ldots \ldots \ldots \ldots$

Office Equipment (PC)

Office Equipment (non-PC) $\ldots \ldots \ldots \ldots \ldots \ldots \ldots \ldots \ldots \ldots$

Other Uses ${ }^{6}$

Delivered Energy
0.71

0.17

0.21

1.16

0.14

0.06

0.17

2.00

6.83
1.57

1.56

0.59

0.72

0.17

0.17

0.21

1.15

0.14

0.07

0.18

2.20

6.96
0.12

0.52

0.17

0.18

0.03

1.20

0.15

0.09

0.21

0.69

3.37

1.33

0.03

0.50

0.21

1.14

3.21

0.20

0.05

0.18

0.42

0.41

0.00

0.00

1.65

0.55

0.72

0.18

0.24

1.20

0.15

0.09

0.21

2.42

7.41
0.12

0.51

0.16

0.18

0.03

1.20

0.15

0.09

0.21

0.70

3.36

1.32

0.03

0.50

0.21

1.15

3.20

0.19

0.05

0.18

0.42

0.41

0.00

0.00

1.64

0.54

0.71

0.18

0.24

1.20

0.15

0.09

0.21

243

7.39
0.10

0.49

0.14

0.18

0.03

1.03

0.14

0.09

0.21

0.70

3.10

1.26

0.03

0.46

0.18

1.15

3.09

0.22

0.05

0.18

0.45

0.41

0.00

0.00

1.58

0.52

0.66

0.18

0.21

1.03

0.14

0.09

0.21

2.44

7.05 
Table B5. Commercial Sector Key Indicators and End-Use Consumption (Continued) (Quadrillion Btu per Year, Unless Otherwise Noted)

\begin{tabular}{c|c|c|c|c|c|c|c|c|c|c|}
\hline \multicolumn{2}{c|}{2010} & \multicolumn{3}{c|}{$\begin{array}{c}\text { Annual Growth } \\
1994-2015 \text { (percent) }\end{array}$} \\
\hline $\begin{array}{c}\text { Slow } \\
\text { Technology }\end{array}$ & Reference & $\begin{array}{c}\text { Rapid } \\
\text { Technology }\end{array}$ & $\begin{array}{c}\text { Slow } \\
\text { Technology }\end{array}$ & $\begin{array}{c}\text { Rapid } \\
\text { Reference }\end{array}$ & $\begin{array}{c}\text { Slow } \\
\text { Technology }\end{array}$ & $\begin{array}{c}\text { Rechnology } \\
\text { Reference }\end{array}$ & $\begin{array}{c}\text { Rapid } \\
\text { Technology }\end{array}$ & $\begin{array}{c}\text { Slow } \\
\text { Technology }\end{array}$ & $\begin{array}{c}\text { Rapid } \\
\text { Reference }\end{array}$ \\
\hline
\end{tabular}

\begin{tabular}{|c|c|c|c|c|c|c|c|c|c|c|c|}
\hline 77.6 & 77.6 & 77.6 & 81.6 & 81.6 & 81.6 & 86.0 & 86.0 & 86.0 & 1.1 & 1.1 & 1.1 \\
\hline 1.7 & 1.7 & 1.7 & 1.8 & 1.8 & 1.8 & 2.0 & 2.0 & 2.0 & 0.5 & 0.5 & 0.5 \\
\hline 79.3 & 79.3 & 79.3 & 83.3 & 83.3 & 83.3 & 88.1 & 88.1 & 88.1 & 1.1 & 1.1 & 1.1 \\
\hline 97.5 & 96.9 & 90.6 & 96.6 & 96.2 & 88.4 & 95.5 & 95.4 & 86.7 & -0.2 & -0.2 & -0.6 \\
\hline 96.5 & 95.2 & 84.0 & 95.1 & 93.8 & 81.8 & 91.0 & 89.5 & 78.7 & -0.4 & -0.5 & -1.1 \\
\hline 194.0 & 192.2 & 174.6 & 191.7 & 190.0 & 170.2 & 186.5 & 184.9 & 165.3 & -0.3 & -0.3 & -0.9 \\
\hline
\end{tabular}

\begin{tabular}{|c|c|c|c|c|c|c|c|c|c|c|c|}
\hline 0.13 & 0.12 & 0.09 & 0.13 & 0.13 & 0.09 & 0.14 & 0.14 & 0.08 & 1.2 & 1.0 & -1.7 \\
\hline 0.52 & 0.50 & 0.46 & 0.53 & 0.50 & 0.44 & 0.54 & 0.50 & 0.43 & 0.1 & -0.2 & -1.0 \\
\hline 0.17 & 0.15 & 0.12 & 0.18 & 0.14 & 0.11 & 0.18 & 0.13 & 0.10 & 0.1 & -1.4 & -2.8 \\
\hline 0.19 & 0.19 & 0.19 & 0.20 & 0.20 & 0.20 & 0.21 & 0.21 & 0.21 & 1.1 & 1.1 & 1.0 \\
\hline 0.03 & 0.03 & 0.03 & 0.03 & 0.03 & 0.03 & 0.03 & 0.03 & 0.03 & -0.4 & -0.5 & -1.3 \\
\hline 1.25 & 1.25 & 0.98 & 1.31 & 1.32 & 0.96 & 1.39 & 1.40 & 0.95 & 0.9 & 0.9 & -0.9 \\
\hline 0.15 & 0.15 & 0.15 & 0.16 & 0.16 & 0.15 & 0.18 & 0.18 & 0.16 & 1.1 & 1.0 & 0.7 \\
\hline 0.10 & 0.10 & 0.10 & 0.11 & 0.11 & 0.11 & 0.12 & 0.12 & 0.12 & 2.6 & 2.6 & 2.6 \\
\hline 0.24 & 0.24 & 0.24 & 0.27 & 0.27 & 0.27 & 0.30 & 0.30 & 0.30 & 2.5 & 2.5 & 2.5 \\
\hline 0.80 & 0.80 & 0.80 & 0.91 & 0.91 & 0.92 & 1.05 & 1.05 & 1.06 & 3.0 & 3.0 & 2.9 \\
\hline 3.58 & 3.55 & 3.15 & 3.83 & 3.77 & 3.26 & 4.14 & 4.06 & 3.42 & 1.3 & 1.2 & 0.4 \\
\hline 1.35 & 1.34 & 1.25 & 1.35 & 1.35 & 1.23 & 1.32 & 1.35 & 1.22 & 0.2 & 0.3 & -0.1 \\
\hline 0.03 & 0.03 & 0.04 & 0.03 & 0.03 & 0.05 & 0.03 & 0.04 & 0.06 & 1.4 & 2.3 & 4.4 \\
\hline 0.52 & 0.52 & 0.48 & 0.54 & 0.54 & 0.51 & 0.55 & 0.57 & 0.54 & 0.6 & 0.8 & 0.6 \\
\hline 0.22 & 0.22 & 0.19 & 0.24 & 0.24 & 0.21 & 0.25 & 0.26 & 0.22 & 1.5 & 1.8 & 1.0 \\
\hline 1.19 & 1.20 & 1.20 & 1.24 & 1.24 & 1.25 & 1.30 & 1.30 & 1.31 & 0.9 & 0.9 & 0.9 \\
\hline 3.31 & 3.31 & 3.16 & 3.40 & 3.42 & 3.25 & 3.44 & 3.51 & 3.35 & 0.6 & 0.7 & 0.5 \\
\hline 0.19 & 0.18 & 0.22 & 0.17 & 0.17 & 0.20 & 0.17 & 0.16 & 0.19 & -0.6 & -1.0 & -0.2 \\
\hline 0.05 & 0.05 & 0.05 & 0.05 & 0.05 & 0.05 & 0.04 & 0.04 & 0.05 & -1.1 & -1.1 & -0.8 \\
\hline 0.18 & 0.18 & 0.18 & 0.18 & 0.18 & 0.18 & 0.19 & 0.19 & 0.19 & 0.4 & 0.4 & 0.4 \\
\hline 0.41 & 0.41 & 0.45 & 0.40 & 0.40 & 0.44 & 0.40 & 0.39 & 0.42 & -0.2 & -0.4 & 0.0 \\
\hline 0.42 & 0.42 & 0.42 & 0.42 & 0.42 & 0.42 & 0.43 & 0.43 & 0.43 & 0.5 & 0.5 & 0.5 \\
\hline 0.00 & 0.00 & 0.00 & 0.00 & 0.00 & 0.00 & 0.00 & 0.00 & 0.00 & 0.7 & 0.7 & 0.7 \\
\hline 0.00 & 0.00 & 0.00 & 0.00 & 0.00 & 0.00 & 0.00 & 0.00 & 0.00 & 0.7 & 0.7 & 0.7 \\
\hline 1.67 & 1.65 & 1.56 & 1.66 & 1.66 & 1.52 & 1.63 & 1.64 & 1.48 & 0.2 & 0.2 & -0.2 \\
\hline 0.55 & 0.54 & 0.50 & 0.56 & 0.53 & 0.49 & 0.57 & 0.54 & 0.49 & 0.1 & -0.1 & -0.6 \\
\hline 0.74 & 0.72 & 0.65 & 0.76 & 0.73 & 0.66 & 0.77 & 0.74 & 0.69 & 0.4 & 0.2 & -0.1 \\
\hline 0.19 & 0.19 & 0.19 & 0.20 & 0.20 & 0.20 & 0.21 & 0.21 & 0.21 & 1.1 & 1.1 & 1.0 \\
\hline 0.25 & 0.26 & 0.22 & 0.27 & 0.27 & 0.23 & 0.28 & 0.29 & 0.25 & 1.2 & 1.5 & 0.7 \\
\hline 1.25 & 1.25 & 0.98 & 1.31 & 1.32 & 0.96 & 1.39 & 1.40 & 0.95 & 0.9 & 0.9 & -0.9 \\
\hline 0.15 & 0.15 & 0.15 & 0.16 & 0.16 & 0.15 & 0.18 & 0.18 & 0.16 & 1.1 & 1.0 & 0.7 \\
\hline 0.10 & 0.10 & 0.10 & 0.11 & 0.11 & 0.11 & 0.12 & 0.12 & 0.12 & 2.6 & 2.6 & 2.6 \\
\hline 0.24 & 0.24 & 0.24 & 0.27 & 0.27 & 0.27 & 0.30 & 0.30 & 0.30 & 2.5 & 2.5 & 2.5 \\
\hline 2.59 & 2.59 & 2.61 & 2.76 & 2.77 & 2.78 & 2.97 & 2.97 & 2.99 & 1.4 & 1.4 & 1.4 \\
\hline 7.73 & 7.68 & 7.18 & 8.05 & 8.02 & 7.37 & 8.41 & 8.40 & 7.63 & 0.9 & 0.9 & 0.4 \\
\hline
\end{tabular}


Table B5. Commercial Sector Key Indicators and End-Use Consumption (Continued) (Quadrillion Btu per Year, Unless Otherwise Noted)

\begin{tabular}{|c|c|c|c|c|c|c|}
\hline \multirow[b]{3}{*}{ Key Indicators and Consumption } & \multirow[b]{3}{*}{1993} & \multirow[b]{3}{*}{1994} & \multirow[b]{3}{*}{1995} & \multicolumn{3}{|c|}{ Projections. } \\
\hline & & & & \multicolumn{3}{|c|}{2000} \\
\hline & & & & $\begin{array}{c}\text { Slow } \\
\text { Technology }\end{array}$ & Reference & $\begin{array}{c}\text { Rapid } \\
\text { Technology }\end{array}$ \\
\hline \multicolumn{7}{|l|}{ Electricity Related Losses by End-Use } \\
\hline 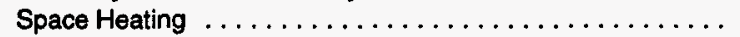 & 0.26 & 0.25 & 0.24 & 0.26 & 0.26 & 0.21 \\
\hline 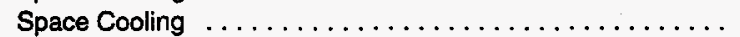 & 1.19 & 1.18 & 1.24 & 1.12 & 1.11 & 1.04 \\
\hline Water Heating $\ldots \ldots \ldots \ldots \ldots \ldots \ldots \ldots \ldots \ldots$ & 0.39 & 0.38 & 0.38 & 0.38 & 0.35 & 0.30 \\
\hline 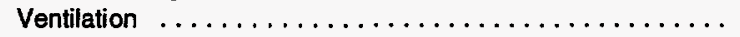 & 0.38 & 0.38 & 0.38 & 0.39 & 0.39 & 0.38 \\
\hline 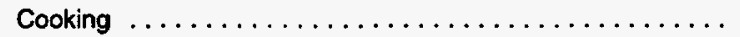 & 0.08 & 0.07 & 0.07 & 0.07 & 0.07 & 0.06 \\
\hline 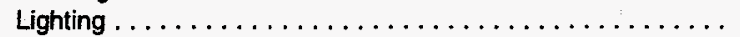 & 2.58 & 2.56 & 2.57 & 2.59 & 2.59 & 2.20 \\
\hline Refrigeration $\ldots \ldots \ldots \ldots \ldots \ldots \ldots \ldots$ & 0.31 & 0.31 & 0.31 & 0.32 & 0.32 & 0.30 \\
\hline Office Equipment $(\mathrm{PC}) \ldots \ldots \ldots \ldots \ldots \ldots$ & 0.12 & 0.15 & 0.16 & 0.19 & 0.19 & 0.19 \\
\hline Office Equipment (non-PC) .. & 0.39 & 0.40 & 0.41 & 0.45 & 0.45 & 0.45 \\
\hline 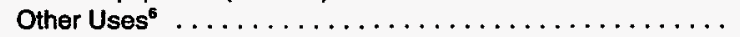 & 1.02 & 1.27 & 1.31 & 1.50 & 1.50 & 1.50 \\
\hline Total Electricity Related Losses . . . . . . . . . . . . . . . . & 6.72 & 6.95 & 7.08 & 7.27 & 7.23 & 6.65 \\
\hline \multicolumn{7}{|l|}{ Total Energy Consumption by End-Use } \\
\hline Space Heating $\ldots \ldots \ldots \ldots \ldots \ldots \ldots \ldots$ & 1.92 & 1.82 & 1.80 & 1.91 & 1.89 & 1.79 \\
\hline Space Cooling $\ldots \ldots \ldots \ldots \ldots \ldots \ldots \ldots \ldots$ & 1.75 & 1.73 & 1.83 & 1.67 & 1.65 & 1.56 \\
\hline 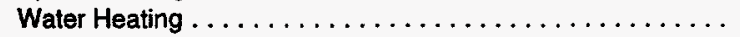 & 1.10 & 1.09 & 1.10 & 1.10 & 1.07 & 0.96 \\
\hline Ventilation $\ldots \ldots \ldots \ldots \ldots \ldots \ldots \ldots \ldots$ & 0.54 & 0.55 & 0.55 & 0.57 & 0.57 & 0.56 \\
\hline 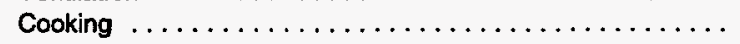 & 0.29 & 0.29 & 0.29 & 0.31 & 0.31 & 0.27 \\
\hline Lighting $\ldots \ldots \ldots \ldots \ldots \ldots \ldots \ldots \ldots$ & 3.74 & 3.72 & 3.73 & 3.79 & 3.80 & 3.23 \\
\hline Refrigeration $\ldots \ldots \ldots \ldots \ldots \ldots \ldots \ldots \ldots$ & 0.45 & 0.45 & 0.46 & 0.46 & 0.46 & 0.45 \\
\hline Office Equipment (PC) $\ldots \ldots \ldots \ldots \ldots \ldots \ldots \ldots$ & 0.18 & 0.22 & 0.23 & 0.28 & 0.28 & 0.28 \\
\hline Office Equipment (non-PC) $\ldots \ldots \ldots \ldots \ldots \ldots \ldots$ & 0.56 & 0.58 & 0.59 & 0.66 & 0.66 & 0.66 \\
\hline Other Uses ${ }^{6} \ldots \ldots \ldots \ldots \ldots \ldots \ldots \ldots \ldots \ldots$ & 3.02 & 3.47 & 3.56 & 3.92 & 3.93 & 3.94 \\
\hline Total $\ldots \ldots \ldots \ldots \ldots \ldots \ldots \ldots \ldots \ldots \ldots$ & 13.55 & 13.91 & 14.14 & 14.68 & 14.62 & 13.70 \\
\hline \multicolumn{7}{|l|}{ Non-Marketed Renewable Fuels } \\
\hline 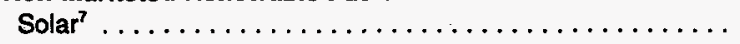 & 0.01 & 0.01 & 0.01 & 0.02 & 0.02 & 0.02 \\
\hline 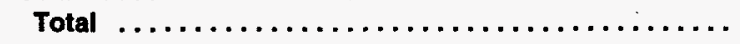 & 0.01 & 0.01 & 0.01 & 0.02 & 0.02 & 0.02 \\
\hline
\end{tabular}


Table B5. Commercial Sector Key Indicators and End-Use Consumption (Continued) (Quadrillion Btu per Year, Unless Otherwise Noted)

\begin{tabular}{|c|c|c|c|c|c|c|c|c|c|c|c|}
\hline \multicolumn{9}{|c|}{ Projections } & \multirow{2}{*}{\multicolumn{3}{|c|}{$\begin{array}{c}\text { Annual Growth } \\
\text { 1994-2015 (percent) }\end{array}$}} \\
\hline \multicolumn{3}{|c|}{2005} & \multicolumn{3}{|c|}{2010} & \multicolumn{3}{|c|}{2015} & & & \\
\hline $\begin{array}{c}\text { Slow } \\
\text { Technology }\end{array}$ & Reference & $\begin{array}{c}\text { Rapid } \\
\text { Technology }\end{array}$ & $\begin{array}{c}\text { Slow } \\
\text { Technology }\end{array}$ & Reference & $\begin{array}{c}\text { Rapid } \\
\text { Technology }\end{array}$ & $\begin{array}{c}\text { Slow } \\
\text { Technology }\end{array}$ & Reference & $\begin{array}{c}\text { Rapid } \\
\text { Technology }\end{array}$ & $\begin{array}{c}\text { Slow } \\
\text { Technology }\end{array}$ & Reference & $\begin{array}{c}\text { Rapid } \\
\text { Technology }\end{array}$ \\
\hline
\end{tabular}

\begin{tabular}{|c|c|c|c|c|c|c|c|c|c|c|c|}
\hline 0.27 & 0.27 & 0.19 & 0.28 & 0.27 & 0.18 & 0.28 & 0.26 & 0.16 & 0.6 & 0.3 & -2.1 \\
\hline 1.12 & 1.08 & 0.98 & 1.09 & 1.04 & 0.93 & 1.04 & 0.98 & 0.87 & -0.6 & -0.9 & -1.4 \\
\hline 0.37 & 0.32 & 0.26 & 0.36 & 0.29 & 0.22 & 0.34 & 0.25 & 0.19 & -0.6 & -2.0 & -3.2 \\
\hline 0.40 & 0.41 & 0.39 & 0.41 & 0.42 & 0.41 & 0.41 & 0.42 & 0.42 & 0.4 & 0.5 & 0.5 \\
\hline 0.07 & 0.07 & 0.05 & 0.06 & 0.06 & 0.05 & 0.06 & 0.06 & 0.05 & -1.1 & -1.1 & -1.7 \\
\hline 2.66 & 2.67 & 2.07 & 2.71 & 2.73 & 2.01 & 2.69 & 2.72 & 1.92 & 0.2 & 0.3 & -1.4 \\
\hline 0.33 & 0.33 & 0.31 & 0.34 & 0.34 & 0.32 & 0.35 & 0.34 & 0.33 & 0.5 & 0.4 & 0.3 \\
\hline 0.21 & 0.21 & 0.21 & 0.22 & 0.22 & 0.23 & 0.22 & 0.22 & 0.23 & 2.0 & 2.0 & 2.2 \\
\hline 0.51 & 0.51 & 0.50 & 0.55 & 0.55 & 0.56 & 0.59 & 0.59 & 0.61 & 1.8 & 1.9 & 2.0 \\
\hline 1.70 & 1.70 & 1.70 & 1.89 & 1.89 & 1.92 & 2.04 & 2.05 & 2.14 & 2.3 & 2.3 & 2.5 \\
\hline 7.65 & 7.55 & 6.66 & 7.93 & 7.82 & 6.82 & 8.02 & 7.88 & 6.93 & 0.7 & 0.6 & 0.0 \\
\hline 1.94 & 1.91 & 1.75 & 1.94 & 1.93 & 1.70 & 1.91 & 1.91 & 1.64 & 0.2 & 0.2 & -0.5 \\
\hline 1.67 & 1.61 & 1.48 & 1.65 & 1.57 & 1.42 & 1.61 & 1.52 & 1.36 & -0.4 & -0.6 & -1.1 \\
\hline 1.11 & 1.04 & 0.91 & 1.12 & 1.02 & 0.88 & 1.11 & 0.99 & 0.88 & 0.1 & -0.4 & -1.0 \\
\hline 0.59 & 0.60 & 0.58 & 0.61 & 0.62 & 0.60 & 0.62 & 0.63 & 0.63 & 0.6 & 0.7 & 0.7 \\
\hline 0.32 & 0.32 & 0.27 & 0.33 & 0.34 & 0.28 & 0.34 & 0.35 & 0.30 & 0.7 & 0.9 & 0.2 \\
\hline 3.91 & 3.92 & 3.05 & 4.02 & 4.05 & 2.97 & 4.08 & 4.12 & 2.87 & 0.4 & 0.5 & -1.2 \\
\hline 0.48 & 0.48 & 0.46 & 0.51 & 0.50 & 0.48 & 0.52 & 0.52 & 0.50 & 0.7 & 0.6 & 0.4 \\
\hline 0.31 & 0.31 & 0.31 & 0.33 & 0.33 & 0.33 & 0.34 & 0.34 & 0.35 & 2.2 & 2.2 & 2.3 \\
\hline 0.74 & 0.74 & 0.74 & 0.82 & 0.82 & 0.83 & 0.89 & 0.89 & 0.91 & 2.1 & 2.1 & 2.2 \\
\hline 4.29 & 4.30 & 4.30 & 4.65 & 4.66 & 4.70 & 5.00 & 5.02 & 5.13 & 1.8 & 1.8 & 1.8 \\
\hline 15.38 & 15.24 & 13.84 & 15.98 & 15.84 & 14.19 & 16.42 & 16.28 & 14.56 & 0.8 & 0.8 & 0.2 \\
\hline 0.03 & 0.03 & 0.03 & 0.03 & 0.03 & 0.03 & 0.04 & 0.04 & 0.04 & 5.7 & 5.7 & 5.7 \\
\hline 0.03 & 0.03 & 0.03 & 0.03 & 0.03 & 0.03 & 0.04 & 0.04 & 0.04 & 5.7 & 5.7 & 5.7 \\
\hline
\end{tabular}

'Includes miscellaneous uses such as service station equipment, district services, automated teller machines, telecommunications equipment, and medical equipment. ${ }^{2}$ Excludes estimated consumption from independent power producers.

Includes miscellaneous uses such as district services, pumps, lighting, emergency electric generators, and manufacturing performed in commercial buildings.

Includes miscellaneous uses such as cooking, district senvices, and emergency electric generators.

Includes residual fuel oil, liquefied pelroleum gas, coal, motor gasoline, and kerosene.

includes miscellaneous uses such as service station equipment, district services, automated teller machines, telecommunications equipment, medical equipment, pumps, lighting,

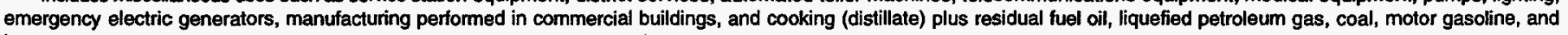
kerosene.

'Includes primary energy displaced by solar thermal water heaters.

Btu = British themal unit.

$\mathrm{PC}=$ Personal computer.

Note: Totals may not equal sum of components due to independent rounding.

Sources: 1993: Energy Information Administration (EIA), State Energy Data Report, DOE/EIA-0214(93) (Washington, DC, July 1995). 1994: ElA, Short-Term Energy Outlook,

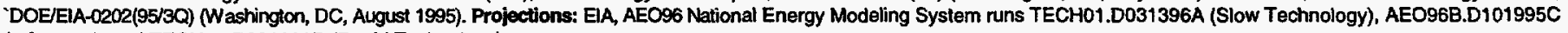
(reference), and TECH04.D031396B (Rapid Technology). 
Table B6. Industrial Sector Key Indicators and Consumption (Quadrillion Btu per Year, Unless Otherwise Noted)

\begin{tabular}{|c|c|c|c|c|c|c|}
\hline \multirow{3}{*}{ Key Indicators and Consumption } & \multirow{3}{*}{1993} & \multirow{3}{*}{1994} & \multirow{3}{*}{1995} & \multicolumn{3}{|c|}{ Projections } \\
\hline & & & & \multicolumn{3}{|c|}{2000} \\
\hline & & & & $\begin{array}{l}\text { Slow } \\
\text { Technology }\end{array}$ & Reference & $\begin{array}{c}\text { Rapid } \\
\text { Technology }\end{array}$ \\
\hline \multicolumn{7}{|l|}{ Key Indicators } \\
\hline \multicolumn{7}{|l|}{ Value of Gross Output (billion 1987 dollars) } \\
\hline 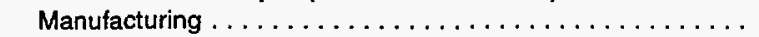 & 2596 & 2784 & 2829 & 3126 & 3127 & 3127 \\
\hline Nonmanufacturing $\ldots \ldots \ldots \ldots \ldots \ldots \ldots \ldots \ldots$ & 888 & 934 & 952 & 1029 & 1030 & 1030 \\
\hline Total $\ldots \ldots \ldots \ldots \ldots \ldots \ldots \ldots \ldots \ldots \ldots$ & 3484 & 3718 & 3782 & 4155 & 4157 & 4157 \\
\hline \multicolumn{7}{|l|}{ Energy Prices (1994 dollars per million Btu) } \\
\hline 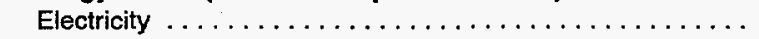 & 14.76 & 14.75 & 14.55 & 14.40 & 14.33 & 14.30 \\
\hline 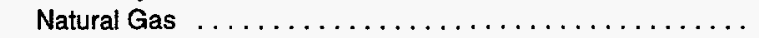 & 2.82 & 2.56 & 2.30 & 2.61 & 2.57 & 2.42 \\
\hline Steam Coal $\ldots \ldots \ldots \ldots \ldots \ldots \ldots \ldots \ldots \ldots$ & 1.45 & 1.45 & 1.38 & 1.35 & 1.30 & 1.23 \\
\hline Residual Oil $\ldots \ldots \ldots \ldots \ldots \ldots \ldots \ldots \ldots \ldots \ldots$ & 2.44 & 2.50 & 2.54 & 2.98 & 2.92 & 2.78 \\
\hline Distillate Oil $\ldots \ldots \ldots \ldots \ldots \ldots \ldots \ldots \ldots$ & 4.87 & 4.59 & 4.64 & 5.44 & 5.33 & 5.13 \\
\hline Liquefied Petroleum Gas . . . . . . . . . . . . . . . . . & 4.87 & 6.11 & 5.84 & 6.76 & 6.66 & 6.66 \\
\hline Motor Gasoline . . . . . . . . . . . . . . . . . & 9.09 & 8.91 & 8.24 & 9.40 & 9.26 & 8.95 \\
\hline Metallurgical Coal $\ldots \ldots \ldots \ldots \ldots \ldots \ldots \ldots \ldots$ & 1.77 & 1.76 & 1.77 & 1.74 & 1.63 & 1.57 \\
\hline \multicolumn{7}{|l|}{ Energy Consumption } \\
\hline \multicolumn{7}{|l|}{ Consumption' (quadrillion Btu per year) } \\
\hline Purchased Electricity $\ldots \ldots \ldots \ldots \ldots \ldots \ldots$ & 3.33 & 3.39 & 3.43 & 3.62 & 3.61 & 3.51 \\
\hline Natural Gas $^{2} \ldots \ldots \ldots \ldots \ldots \ldots \ldots \ldots \ldots$ & 9.07 & 9.34 & 9.76 & 10.74 & 10.49 & 10.26 \\
\hline Steam Coal $\ldots \ldots \ldots \ldots \ldots \ldots \ldots \ldots \ldots \ldots$ & 1.66 & 1.69 & 1.67 & 1.98 & 1.73 & 1.56 \\
\hline Metallurgical Coal and Coke $^{3} \ldots \ldots \ldots \ldots \ldots \ldots$ & 0.86 & 0.86 & 0.84 & 0.90 & 0.74 & 0.70 \\
\hline Residual Fuel $\ldots \ldots \ldots \ldots \ldots \ldots \ldots \ldots \ldots \ldots$ & 0.45 & 0.47 & 0.47 & 0.51 & 0.48 & 0.45 \\
\hline Distillate $\ldots \ldots \ldots \ldots \ldots \ldots \ldots \ldots \ldots$ & 1.10 & 1.15 & 1.16 & 1.22 & 1.21 & 1.17 \\
\hline Liquefied Petroleum Gas . . . . . . . . . . . . . . . . & 1.79 & 1.87 & 1.92 & 1.97 & 1.97 & 1.91 \\
\hline Petrochemical Feedstocks $\ldots \ldots \ldots \ldots \ldots \ldots \ldots \ldots$ & 1.19 & 1.25 & 1.25 & 1.27 & 1.27 & 1.24 \\
\hline Other Petroleum ${ }^{4} \ldots \ldots \ldots \ldots \ldots \ldots \ldots \ldots \ldots$ & 3.87 & 4.09 & 4.11 & 4.29 & 4.27 & 4.21 \\
\hline Renewables ${ }^{5} \ldots \ldots \ldots \ldots \ldots \ldots \ldots \ldots \ldots \ldots \ldots$ & 2.08 & 2.16 & 2.19 & 2.36 & 2.36 & 2.36 \\
\hline Delivered Energy $\ldots \ldots \ldots \ldots \ldots \ldots \ldots \ldots \ldots \ldots \ldots$ & 25.42 & 26.27 & 26.80 & 28.87 & 28.15 & 27.38 \\
\hline Electricity Related Losses . . . . . . . . . . . . . . . . . & 7.43 & 7.53 & 7.58 & 7.80 & 7.78 & 7.53 \\
\hline Total $\ldots \ldots \ldots \ldots \ldots \ldots \ldots \ldots \ldots \ldots$ & 32.85 & 33.80 & 34.38 & 36.67 & 35.93 & 34.91 \\
\hline \multicolumn{7}{|l|}{$\begin{array}{l}\text { Consumption per Unit of Output } \\
\text { (thousand Btu per } 1987 \text { dollars) }\end{array}$} \\
\hline Purchased Electricity $\ldots \ldots \ldots \ldots \ldots \ldots \ldots$ & 0.96 & 0.91 & 0.91 & 0.87 & 0.87 & 0.85 \\
\hline 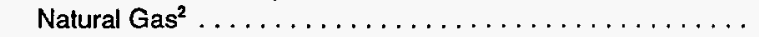 & 2.60 & 2.51 & 2.58 & 2.59 & 2.52 & 2.47 \\
\hline Steam Coal $\ldots \ldots \ldots \ldots \ldots \ldots \ldots \ldots \ldots \ldots \ldots \ldots$ & 0.48 & 0.45 & 0.44 & 0.48 & 0.42 & 0.38 \\
\hline Metallurgical Coal and Coke ${ }^{3} \ldots$ & 0.25 & 0.23 & 0.22 & 0.22 & 0.18 & 0.17 \\
\hline Residual Fuel $\ldots \ldots \ldots \ldots \ldots \ldots \ldots \ldots \ldots \ldots \ldots$ & 0.13 & 0.13 & 0.12 & 0.12 & 0.11 & 0.11 \\
\hline 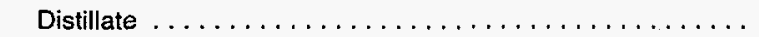 & 0.32 & 0.31 & 0.31 & 0.29 & 0.29 & 0.28 \\
\hline Liquefied Petroleum Gas . . . . . . . . . . . . . . . . . . & 0.52 & 0.50 & 0.51 & 0.47 & 0.47 & 0.46 \\
\hline Petrochemical Feedstocks $\ldots \ldots \ldots \ldots \ldots \ldots \ldots \ldots \ldots$ & 0.34 & 0.34 & 0.33 & 0.31 & 0.31 & 0.30 \\
\hline Other Petroleum ${ }^{4} \ldots \ldots \ldots \ldots \ldots \ldots \ldots \ldots \ldots \ldots$ & 1.11 & 1.10 & 1.09 & 1.03 & 1.03 & 1.01 \\
\hline Renewables $^{5} \ldots \ldots \ldots \ldots \ldots \ldots \ldots \ldots \ldots \ldots \ldots \ldots$ & 0.60 & 0.58 & 0.58 & 0.57 & 0.57 & 0.57 \\
\hline Delivered Energy $\ldots \ldots \ldots \ldots \ldots \ldots \ldots \ldots \ldots \ldots$ & 7.30 & 7.07 & 7.09 & 6.95 & 6.77 & 6.59 \\
\hline Electricity Related Losses . . . . . . . . . . . . . . . . . . & 2.13 & 2.02 & 2.00 & 1.88 & 1.87 & 1.81 \\
\hline Total $\ldots \ldots \ldots \ldots \ldots \ldots \ldots \ldots \ldots \ldots$ & 9.43 & 9.09 & 9.09 & 8.83 & 8.64 & 8.40 \\
\hline
\end{tabular}

${ }^{1}$ Fuel consumption includes consumption for cogeneration.

${ }^{2}$ Includes lease and plant fuel.

Includes net coke coal imports.

Includes petroleum coke, asphalt, road oil, lubricants, motor gasoline, still gas, and miscellaneous petroleum products.

5Includes solar, geothermal, and biomass energy. 
Table B6. Industrial Sector Key Indicators and Consumption (Continued) (Quadrillion Btu per Year, Unless Otherwise Noted)

\begin{tabular}{|c|c|c|c|c|c|c|c|c|c|c|c|}
\hline \multicolumn{9}{|c|}{ Projections } & \multirow{2}{*}{\multicolumn{3}{|c|}{$\begin{array}{c}\text { Annual Growth } \\
\text { 1994-2015 (percent) }\end{array}$}} \\
\hline \multicolumn{3}{|c|}{2005} & \multicolumn{3}{|c|}{2010} & \multicolumn{3}{|c|}{2015} & & & \\
\hline $\begin{array}{c}\text { Slow } \\
\text { Technology }\end{array}$ & Reference & $\begin{array}{c}\text { Rapid } \\
\text { Technology }\end{array}$ & $\begin{array}{c}\text { Slow } \\
\text { Technology }\end{array}$ & Reference & $\begin{array}{c}\text { Rapid } \\
\text { Technology }\end{array}$ & $\begin{array}{c}\text { Slow } \\
\text { Technology }\end{array}$ & Reference & $\begin{array}{c}\text { Rapid } \\
\text { Technology }\end{array}$ & $\begin{array}{c}\text { Slow } \\
\text { Technology }\end{array}$ & Reference & $\begin{array}{c}\text { Rapid } \\
\text { Technology }\end{array}$ \\
\hline
\end{tabular}

\begin{tabular}{|c|c|c|c|c|c|c|c|c|c|c|c|}
\hline 3507 & 3508 & 3510 & 3808 & 3811 & 3815 & 4108 & 4120 & 4126 & 1.9 & 1.9 & 1.9 \\
\hline 1094 & 1099 & 1107 & 1142 & 1155 & 1179 & 1199 & 1226 & 1280 & 1.2 & 1.3 & 1.5 \\
\hline 4601 & 4607 & 4617 & 4949 & 4966 & 4994 & 5306 & 5346 & 5407 & 1.7 & 1.7 & 1.8 \\
\hline 14.52 & 14.37 & 14.12 & 14.62 & 14.14 & 13.61 & 14.97 & 14.07 & 13.07 & 0.1 & -0.2 & -0.6 \\
\hline 2.88 & 2.64 & 2.19 & 3.59 & 2.76 & 2.02 & 4.79 & 3.20 & 2.14 & 3.0 & 1.1 & -0.9 \\
\hline 1.45 & 1.33 & 1.21 & 1.48 & 1.33 & 1.19 & 1.50 & 1.31 & 1.19 & 0.2 & -0.5 & -0.9 \\
\hline 3.53 & 3.35 & 3.00 & 3.95 & 3.66 & 3.14 & 4.42 & 3.92 & 3.26 & 2.7 & 2.2 & 1.3 \\
\hline 5.91 & 5.72 & 5.27 & 6.39 & 6.04 & 5.40 & 6.79 & 6.18 & 5.34 & 1.9 & 1.4 & 0.7 \\
\hline 7.21 & 7.13 & 6.70 & 7.94 & 7.50 & 6.54 & 8.54 & 7.59 & 5.24 & 1.6 & 1.0 & -0.7 \\
\hline 10.18 & 9.79 & 9.00 & 10.83 & 10.12 & 9.01 & 11.06 & 10.09 & 8.67 & 1.0 & 0.6 & -0.1 \\
\hline 1.85 & 1.66 & 1.58 & 1.85 & 1.66 & 1.56 & 1.87 & 1.64 & 1.54 & 0.3 & -0.3 & -0.6 \\
\hline
\end{tabular}

\begin{tabular}{|c|c|c|c|c|c|c|c|c|c|c|c|}
\hline 3.91 & 3.86 & 3.63 & 4.14 & 4.04 & 3.67 & 4.36 & 4.23 & 3.69 & 1.2 & 1.1 & 0.4 \\
\hline 11.46 & 11.12 & 10.55 & 11.79 & 11.55 & 10.71 & 11.76 & 11.86 & 10.53 & 1.1 & 1.1 & 0.6 \\
\hline 2.27 & 1.76 & 1.39 & 2.71 & 1.83 & 1.29 & 3.26 & 1.96 & 1.25 & 3.2 & 0.7 & -1.4 \\
\hline 0.88 & 0.66 & 0.58 & 0.85 & 0.59 & 0.49 & 0.83 & 0.52 & 0.40 & -0.2 & -2.4 & -3.5 \\
\hline 0.54 & 0.49 & 0.44 & 0.57 & 0.49 & 0.36 & 0.60 & 0.52 & 0.40 & 1.1 & 0.4 & -0.8 \\
\hline 1.29 & 1.28 & 1.15 & 1.35 & 1.34 & 1.06 & 1.59 & 1.40 & 0.96 & 1.6 & 0.9 & -0.9 \\
\hline 2.10 & 2.11 & 1.89 & 2.19 & 2.19 & 1.75 & 2.29 & 2.30 & 1.62 & 1.0 & 1.0 & -0.7 \\
\hline 1.36 & 1.36 & 1.22 & 1.42 & 1.42 & 1.12 & 1.48 & 1.48 & 0.98 & 0.8 & 0.8 & -1.2 \\
\hline 4.46 & 4.38 & 4.07 & 4.64 & 4.44 & 3.85 & 4.82 & 4.55 & 3.61 & 0.8 & 0.5 & -0.6 \\
\hline 2.56 & 2.56 & 2.57 & 2.70 & 2.71 & 2.71 & 2.85 & 2.86 & 2.86 & 1.3 & 1.3 & 1.3 \\
\hline 30.83 & 29.58 & 27.49 & 32.35 & 30.60 & 26.99 & 33.84 & 31.68 & 26.30 & 1.2 & 0.9 & 0.0 \\
\hline 8.35 & 8.22 & 7.68 & 8.56 & 8.38 & 7.67 & 8.46 & 8.22 & 7.47 & 0.6 & 0.4 & 0.0 \\
\hline 39.18 & $\mathbf{3 7 . 8 1}$ & 35.16 & 40.92 & 38.98 & 34.66 & 42.30 & 39.89 & 33.77 & 1.1 & 0.8 & 0.0 \\
\hline
\end{tabular}

\begin{tabular}{|c|c|c|c|c|c|c|c|c|c|c|c|}
\hline 0.85 & 0.84 & 0.79 & 0.84 & 0.81 & 0.73 & 0.82 & 0.79 & 0.68 & -0.5 & -0.7 & -1.4 \\
\hline 2.49 & 2.41 & 2.29 & 2.38 & 2.33 & 2.14 & 2.22 & 2.22 & 1.95 & -0.6 & -0.6 & -1.2 \\
\hline 0.49 & 0.38 & 0.30 & 0.55 & 0.37 & 0.26 & 0.61 & 0.37 & 0.23 & 1.5 & -1.0 & -3.2 \\
\hline 0.19 & 0.14 & 0.13 & 0.17 & 0.12 & 0.10 & 0.16 & 0.10 & 0.07 & -1.9 & -4.1 & -5.3 \\
\hline 0.12 & 0.11 & 0.10 & 0.12 & 0.10 & 0.07 & 0.11 & 0.10 & 0.07 & -0.6 & -1.3 & -2.6 \\
\hline 0.28 & 0.28 & 0.25 & 0.27 & 0.27 & 0.21 & 0.30 & 0.26 & 0.18 & -0.1 & -0.8 & -2.6 \\
\hline 0.46 & 0.46 & 0.41 & 0.44 & 0.44 & 0.35 & 0.43 & 0.43 & 0.30 & -0.7 & -0.8 & -2.4 \\
\hline 0.29 & 0.29 & 0.26 & 0.29 & 0.29 & 0.22 & 0.28 & 0.28 & 0.18 & -0.9 & -0.9 & -2.9 \\
\hline 0.97 & 0.95 & 0.88 & 0.94 & 0.89 & 0.77 & 0.91 & 0.85 & 0.67 & -0.9 & -1.2 & -2.3 \\
\hline 0.56 & 0.56 & 0.56 & 0.55 & 0.55 & 0.54 & 0.54 & 0.54 & 0.53 & -0.4 & -0.4 & -0.5 \\
\hline 6.70 & 6.42 & 5.95 & 6.54 & 6.16 & 5.41 & 6.38 & 5.93 & 4.86 & -0.5 & -0.8 & -1.8 \\
\hline 1.82 & 1.79 & 1.66 & 1.73 & 1.69 & 1.54 & 1.59 & 1.54 & 1.38 & -1.1 & -1.3 & -1.8 \\
\hline 8.52 & 8.21 & 7.62 & 8.27 & 7.85 & 6.94 & 7.97 & 7.46 & 6.25 & -0.6 & -0.9 & -1.8 \\
\hline
\end{tabular}

Btu = British thermal unit.

Note: Totals may not equal sum of components due to independent rounding.

Sources: 1993 prices for gasoline and distillate are based on prices in the Energy Information Administration (EIA), Petroleum Marketing Annual 1993, DOE/EIA-0487(93) Washington, DC, July 1994). 1994 prices for gasoline and distillate are based on prices in various issues of EIA, Petroleum Marketing Monthly, DOE/EIA-0380(94/1-12) (Washington, DC, 1994). 1993 and 1994 coal prices: EIA, Monthly Energy Review, DOE/EIA-0035(95/08) (Washington, DC, August 1995). 1993 and 1994 natural gas and electricity prices: EIA,

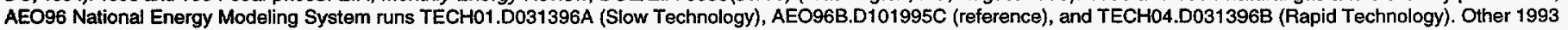

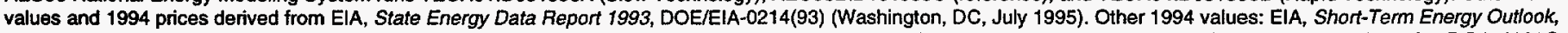

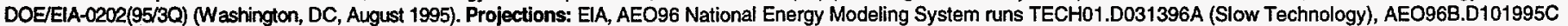
(reference), and TECH04.D031396B (Rapid Technology). 
Table B7. Transportation Sector Key Indicators and Delivered Energy Consumption

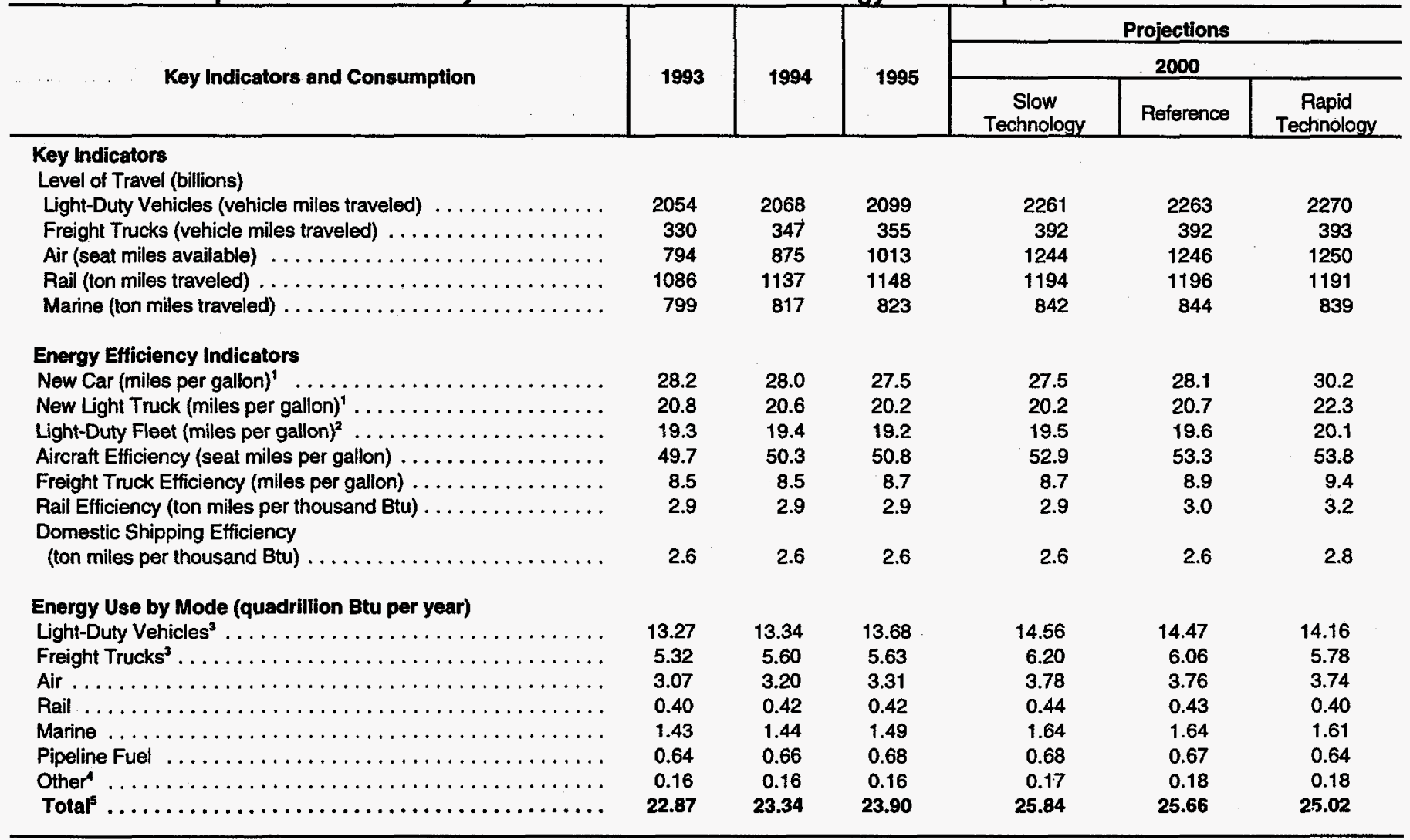


Table B7. Transportation Sector Key Indicators and Delivered Energy Consumption (Continued)

\begin{tabular}{|c|c|c|c|c|c|c|c|c|c|c|c|}
\hline \multicolumn{9}{|c|}{ Projections } & \multirow{2}{*}{\multicolumn{3}{|c|}{$\begin{array}{l}\text { Annual Growth } \\
\text { 1994-2015 (percent) }\end{array}$}} \\
\hline \multicolumn{3}{|c|}{2005} & \multicolumn{3}{|c|}{2010} & \multicolumn{3}{|c|}{2015} & & & \\
\hline $\begin{array}{c}\text { Slow } \\
\text { Technology }\end{array}$ & Reference & $\begin{array}{c}\text { Rapid } \\
\text { Technology }\end{array}$ & $\begin{array}{c}\text { Slow } \\
\text { Technology }\end{array}$ & Reference & $\begin{array}{c}\text { Rapid } \\
\text { Technology }\end{array}$ & $\mid \begin{array}{c}\text { Slow } \\
\text { Technology }\end{array}$ & Reference & $\begin{array}{c}\text { Rapid } \\
\text { Technology }\end{array}$ & $\begin{array}{c}\text { Slow } \\
\text { Technology }\end{array}$ & Reference & $\begin{array}{c}\text { Rapid } \\
\text { Technology }\end{array}$ \\
\hline 2448 & 2457 & 2473 & 2609 & 2629 & 2654 & 2742 & 2774 & 2807 & 1.4 & 1.4 & 1.5 \\
\hline 428 & 428 & 430 & 456 & 458 & 460 & 486 & 489 & 493 & 1.6 & 1.6 & 1.7 \\
\hline 877 & 889 & 905 & 901 & 933 & 1002 & 918 & 989 & 1160 & 0.6 & 0.9 & 1.7 \\
\hline 27.5 & 30.5 & 33.4 & 27.5 & 31.9 & 36.7 & 27.5 & 33.1 & 40.2 & -0.1 & 0.8 & 1.7 \\
\hline 20.2 & 21.9 & 24.6 & 20.3 & 23.8 & 27.1 & 20.3 & 25.1 & 29.7 & -0.1 & 1.0 & 1.8 \\
\hline 19.5 & 20.2 & 21.1 & 19.4 & 20.8 & 22.5 & 19.5 & 21.8 & 24.3 & 0.0 & 0.6 & 1.1 \\
\hline 15.79 & 15.26 & 14.61 & 16.86 & 15.78 & 14.68 & 17.65 & 15.92 & 14.41 & 1.3 & 0.8 & 0.4 \\
\hline 6.76 & 6.52 & 5.88 & 7.19 & 6.88 & 5.85 & 7.65 & 7.29 & 5.83 & 1.5 & 1.3 & 0.2 \\
\hline 4.24 & 4.17 & 4.10 & 4.60 & 4.48 & 4.32 & 4.94 & 4.75 & 4.47 & 2.1 & 1.9 & 1.6 \\
\hline 0.46 & 0.45 & 0.40 & 0.48 & 0.47 & 0.40 & 0.49 & 0.49 & 0.42 & 0.8 & 0.8 & 0.0 \\
\hline 1.81 & 1.82 & 1.78 & 1.99 & 2.00 & 1.95 & 2.14 & 2.18 & 2.13 & 1.9 & 2.0 & 1.9 \\
\hline 0.71 & 0.71 & 0.65 & 0.78 & 0.76 & 0.68 & 0.85 & 0.86 & 0.77 & 1.2 & 1.3 & 0.8 \\
\hline 0.19 & 0.19 & 0.19 & 0.19 & 0.19 & 0.20 & 0.20 & 0.20 & 0.21 & 1.1 & 1.2 & 1.2 \\
\hline 28.18 & 27.47 & 26.09 & 30.19 & 28.86 & 26.59 & 31.89 & 29.90 & 26.74 & 1.5 & 1.2 & 0.6 \\
\hline
\end{tabular}

'Environmental Protection Agency rated miles per gallon.

${ }^{2}$ Combined car and light truck "on-the-road" estimate.

includes light-duty trucks used for freight.

includes lubricants and aviation gasoline.

Total will not equal sum of components due to light-duty freight trucks included in both light-duty vehicle and freight truck consumption.

Btu = British thermal unit.

Note: Totals may not equal sum of components due to independent rounding.

Sources:1993 pipeline fuel consumption: Energy Information Administration (EIA), Natural Gas Annual 1993, DOE/EIA-0131(93) (Washington, DC, October 1994). Other 1993 values: Federal Highway Administration, Highway Statistics 1993 (Washington, DC, 1993); Oak Ridge National Laboratory, Transportation Energy Data Book: 12, 13, 14, and 15, (Oak Ridge, TN, May 1995); Federal Aviation Administration (FAA), FAA Aviation Forecasts Fiscal Years 1993-2004; National Highway Traffic and Safety Administration, Summany of Fuel Economy Performance, (Washington, DC, February 1994); ElA, Residential Transportation Energy Consumption Survey 1991, DOE/EIA-0464(91) (Washington, DC, December 1993); Argonne National Laboratory, FRATE Model 1990; and EIA, State Energy Data Report 1993, DOE/EIA-0214(93) (Washington, DC, July 1995). 1994 pipeline fuel consumption: EIA, Natural Gas Monthy, DOE/EIA-0130(95/6) Washington, DC, June 1995). Other 1994 values: FAA, FAA Aviation Forecasts Fiscal Years 1993-2004, (Washington, DC, February 1994); EIA, Short-Term Energy Outlook, DOE/EIA-0202(95/3Q) (Washington, DC, August 1995); EIA, Fuel Oil and Kerosene Sales 1993, DOE/EIA-0535(92) (Washington, DC, September 1994); and United States Department of Defense, Defense Fuel Supply Center. Projections: EIA, AEO96 National Energy Modeling System runs TECHO1.D031396A (Slow Technology), AEO96B.D101995C (reference), and TECH04.D031396B (Rapid Technology). 
Table B8. Electricity Supply, Disposition, and Prices (Billion Kilowatthours, Unless Otherwise Noted)

\begin{tabular}{|c|c|c|c|c|c|c|}
\hline \multirow{3}{*}{ Supply, Disposition, and Prices } & \multirow{3}{*}{1993} & \multirow{3}{*}{1994} & \multirow{3}{*}{1995} & \multicolumn{3}{|c|}{ Projections } \\
\hline & & & & \multicolumn{3}{|c|}{2000} \\
\hline & & & & $\begin{array}{c}\text { Slow } \\
\text { Technology }\end{array}$ & Reference & $\begin{array}{c}\text { Rapid } \\
\text { Technology }\end{array}$ \\
\hline \multicolumn{7}{|l|}{$\begin{array}{l}\text { Generation by Fuel Type } \\
\text { Electric Utilities }\end{array}$} \\
\hline Coal $\ldots \ldots \ldots \ldots \ldots \ldots \ldots \ldots \ldots \ldots$ & 1639 & 1635 & 1654 & 1780 & 1778 & 1725 \\
\hline Petroleum $\ldots \ldots \ldots \ldots \ldots \ldots \ldots \ldots \ldots \ldots \ldots \ldots \ldots$ & 100 & 91 & 66 & 66 & 63 & 46 \\
\hline Natural Gas $\ldots \ldots \ldots \ldots \ldots \ldots \ldots \ldots \ldots \ldots$ & 259 & 291 & 312 & 308 & 299 & 217 \\
\hline Nuclear Power $\ldots \ldots \ldots \ldots \ldots \ldots \ldots \ldots \ldots \ldots \ldots$ & 610 & 640 & 658 & 664 & 664 & 664 \\
\hline Pumped Storage $\ldots \ldots \ldots \ldots \ldots \ldots \ldots \ldots \ldots$ & -2 & -3 & -3 & -3 & -3 & -3 \\
\hline Renewable Sources' . . . . . . . . . . . . . . . . . . . & 277 & 256 & 290 & 294 & 294 & 295 \\
\hline Total $\ldots \ldots \ldots \ldots \ldots \ldots \ldots \ldots \ldots \ldots \ldots$ & 2883 & 2911 & 2977 & 3108 & 3095 & 2944 \\
\hline \multicolumn{7}{|l|}{ Nonutilities (excluding cogenerators) ${ }^{2}$} \\
\hline Coal $\ldots \ldots \ldots \ldots \ldots \ldots \ldots \ldots \ldots \ldots \ldots$ & 10 & 12 & 13 & 17 & 15 & 15 \\
\hline Petroleum $\ldots \ldots \ldots \ldots \ldots \ldots \ldots \ldots \ldots \ldots \ldots \ldots \ldots$ & 1 & 2 & 3 & 3 & 3 & 3 \\
\hline Natural Gas $\ldots \ldots \ldots \ldots \ldots \ldots \ldots \ldots \ldots$ & 13 & 12 & 15 & 30 & 28 & 25 \\
\hline Renewable Sources' $\ldots \ldots \ldots \ldots \ldots \ldots \ldots$ & 49 & 52 & 56 & 67 & 67 & 68 \\
\hline Total $\ldots \ldots \ldots \ldots \ldots \ldots \ldots \ldots \ldots \ldots \ldots \ldots \ldots$ & 73 & 78 & 86 & 116 & 114 & 111 \\
\hline Sales to Utilities $\ldots \ldots \ldots \ldots \ldots \ldots \ldots \ldots \ldots \ldots \ldots$ & 47 & 51 & 59 & 88 & 86 & 83 \\
\hline Generation for Own Use $\ldots \ldots \ldots \ldots \ldots \ldots \ldots$ & 26 & 27 & 27 & 28 & 28 & 28 \\
\hline \multicolumn{7}{|l|}{ Cogenerators $^{3}$} \\
\hline Coal $\ldots \ldots \ldots \ldots \ldots \ldots \ldots \ldots \ldots \ldots \ldots$ & 43 & 47 & 43 & 48 & 48 & 48 \\
\hline Petroleum $\ldots \ldots \ldots \ldots \ldots \ldots \ldots \ldots \ldots \ldots$ & 7 & 7 & 5 & 5 & 5 & 5 \\
\hline Natural Gas $\ldots \ldots \ldots \ldots \ldots \ldots \ldots \ldots \ldots \ldots$ & 154 & 171 & 176 & 191 & 191 & 191 \\
\hline Other Gaseous Fuels ${ }^{4} \ldots \ldots \ldots \ldots \ldots \ldots \ldots \ldots$ & 15 & 14 & 17 & 17 & 17 & 17 \\
\hline Renewable Sources ${ }^{t} \ldots \ldots \ldots \ldots \ldots \ldots \ldots \ldots \ldots \ldots$ & 38 & 40 & 40 & 43 & 43 & 43 \\
\hline Other ${ }^{5} \ldots \ldots \ldots \ldots \ldots \ldots \ldots \ldots \ldots$ & 3 & 3 & 3 & 3 & 3 & 3 \\
\hline Total $\ldots \ldots \ldots \ldots \ldots \ldots \ldots \ldots \ldots \ldots$ & 259 & 282 & 284 & 307 & 307 & 306 \\
\hline Sales to Utilities $\ldots \ldots \ldots \ldots \ldots \ldots \ldots \ldots \ldots$ & 137 & 153 & 148 & 163 & 163 & 163 \\
\hline Generation for Own Use . . . . . . . . . . . . . . & 123 & 129 & 136 & 143 & 144 & 143 \\
\hline Net Imports $\ldots \ldots \ldots \ldots \ldots \ldots \ldots \ldots \ldots \ldots \ldots \ldots \ldots$ & 28 & 45 & 39 & 40 & 40 & 40 \\
\hline \multicolumn{7}{|l|}{ Electricity Sales by Sector } \\
\hline Residential $\ldots \ldots \ldots \ldots \ldots \ldots \ldots \ldots \ldots \ldots \ldots \ldots$ & 995 & 1006 & 1024 & 1091 & 1084 & 1045 \\
\hline Commercial $\ldots \ldots \ldots \ldots \ldots \ldots \ldots \ldots \ldots \ldots \ldots$ & 884 & 917 & 939 & 989 & 984 & 910 \\
\hline Industrial $\ldots \ldots \ldots \ldots \ldots \ldots \ldots \ldots \ldots \ldots \ldots \ldots \ldots \ldots \ldots$ & 977 & 992 & 1004 & 1061 & 1059 & 1030 \\
\hline Transportation $\ldots \ldots \ldots \ldots \ldots \ldots \ldots \ldots \ldots \ldots$ & 6 & 6 & 6 & 9 & 9 & 9 \\
\hline 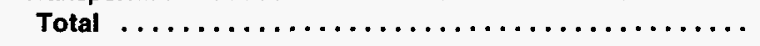 & 2861 & 2921 & 2973 & 3151 & 3137 & 2994 \\
\hline \multicolumn{7}{|l|}{ End-Use Prices (1994 cents per kilowatthour) ${ }^{6}$} \\
\hline Residential $\ldots \ldots \ldots \ldots \ldots \ldots \ldots \ldots \ldots \ldots \ldots \ldots \ldots$ & 8.5 & 8.4 & 8.4 & 8.4 & 8.4 & 8.3 \\
\hline Commercial $\ldots \ldots \ldots \ldots \ldots \ldots \ldots \ldots \ldots$ & 7.9 & 7.9 & 7.9 & 7.7 & 7.7 & 7.7 \\
\hline 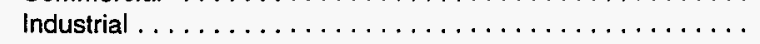 & 5.0 & 5.0 & 5.0 & 4.9 & 4.9 & 4.9 \\
\hline Transportation $\ldots \ldots \ldots \ldots \ldots \ldots \ldots \ldots \ldots$ & 5.0 & 5.0 & 5.1 & 5.2 & 5.2 & 5.3 \\
\hline All Sectors Average $\ldots \ldots \ldots \ldots \ldots \ldots \ldots \ldots$ & 7.1 & 7.1 & 7.1 & 7.0 & 7.0 & 6.9 \\
\hline \multicolumn{7}{|l|}{ Price Components (1994 cents per kilowatthour) } \\
\hline Capital Component $\ldots \ldots \ldots \ldots \ldots \ldots \ldots \ldots \ldots$ & 2.7 & 2.8 & 2.7 & 2.6 & 2.6 & 2.7 \\
\hline Fuel Component $\ldots \ldots \ldots \ldots \ldots \ldots \ldots \ldots \ldots \ldots$ & 1.2 & 1.2 & 1.2 & 1.1 & 1.1 & 1.0 \\
\hline Operation and Maintenance Component $\ldots \ldots \ldots \ldots \ldots \ldots$ & 2.8 & 2.8 & 2.8 & 2.8 & 2.8 & 2.8 \\
\hline Wholesale Power Cost $\ldots \ldots \ldots \ldots \ldots \ldots \ldots \ldots$ & 0.4 & 0.4 & 0.4 & 0.4 & 0.4 & 0.4 \\
\hline 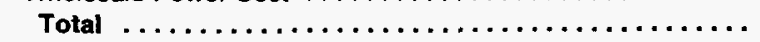 & 7.1 & 7.1 & 7.1 & 7.0 & 7.0 & 6.9 \\
\hline
\end{tabular}

'Includes conventional hydroelectric, geothermal, wood, wood waste, municipal solid waste, other biomass, solar and wind power.

includes generation at all nonutilities except for cogenerators. Includes small power producers, exempt wholesale generators, and generators at industrial and commercial facilities which provide electricity for on-site use and for sales to utilities.

${ }^{3}$ Includes cogeneration at facilities whose primary function is not electricity production. Includes sales to utilities and generation for own use.

"Other gaseous fuels include refinery and still gas.

'Other includes hydrogen, sulfur, batteries, chemicals, fish oil, and spent sulfite liquor.

sprices represent average revenue per kilowathour. 
Table B8. Electricity Supply, Disposition, and Prices (Continued) (Billion Kilowatthours, Unless Otherwise Noted)

\begin{tabular}{|c|c|c|c|c|c|c|c|c|c|c|c|}
\hline \multicolumn{9}{|c|}{ Projections } & \multirow{2}{*}{\multicolumn{3}{|c|}{$\begin{array}{l}\text { Annual Growth } \\
\text { 1994-2015 (percent) }\end{array}$}} \\
\hline \multicolumn{3}{|c|}{2005} & \multicolumn{3}{|c|}{2010} & \multicolumn{3}{|c|}{2015} & & & \\
\hline $\begin{array}{c}\text { Slow } \\
\text { Technology }\end{array}$ & Reference & $\begin{array}{c}\text { Rapid } \\
\text { Technology }\end{array}$ & $\begin{array}{c}\text { Slow } \\
\text { Technology }\end{array}$ & Reference & $\begin{array}{c}\text { Rapid } \\
\text { Technology }\end{array}$ & $\begin{array}{c}\text { Slow } \\
\text { Technology }\end{array}$ & Reference & $\begin{array}{c}\text { Rapid } \\
\text { Technology }\end{array}$ & $\begin{array}{c}\text { Slow } \\
\text { Technology }\end{array}$ & Reference & $\begin{array}{c}\text { Rapid } \\
\text { Technology }\end{array}$ \\
\hline
\end{tabular}

\begin{tabular}{|c|c|c|c|c|c|c|c|c|c|c|c|}
\hline 1928 & 1909 & 1822 & 2017 & 1995 & 1898 & 2131 & 2054 & 1949 & 1.3 & 1.1 & 0.8 \\
\hline 98 & 89 & 56 & 121 & 111 & 73 & 148 & 104 & 93 & 2.3 & 0.6 & 0.1 \\
\hline 382 & 363 & 237 & 464 & 446 & 283 & 554 & 590 & 445 & 3.1 & 3.4 & 2.0 \\
\hline 649 & 649 & 649 & 610 & 610 & 610 & 434 & 434 & 434 & -1.8 & -1.8 & -1.8 \\
\hline-3 & -3 & -3 & -3 & -3 & -3 & -3 & -3 & -3 & 0.3 & 0.3 & 0.3 \\
\hline 297 & 297 & 296 & 298 & 299 & 297 & 306 & 308 & 302 & 0.9 & 0.9 & 0.8 \\
\hline 3351 & 3304 & 3058 & 3508 & 3458 & 3159 & 3570 & 3487 & 3219 & 1.0 & 0.9 & 0.5 \\
\hline
\end{tabular}

\begin{tabular}{|c|c|c|c|c|c|c|c|c|c|c|c|}
\hline 21 & 17 & 16 & 32 & 23 & 18 & 83 & 37 & 21 & 9.6 & 5.5 & 2.7 \\
\hline 5 & 5 & 3 & 8 & 7 & 3 & 13 & 9 & 8 & 10.5 & 8.5 & 7.7 \\
\hline 53 & 51 & 27 & 161 & 117 & 35 & 358 & 332 & 122 & 17.5 & 17.1 & 11.6 \\
\hline 71 & 73 & 72 & 79 & 82 & 78 & 101 & 110 & 94 & 3.2 & 3.7 & 2.9 \\
\hline 150 & 145 & 118 & 280 & 229 & 135 & 555 & 489 & 244 & 9.8 & 9.2 & 5.6 \\
\hline 121 & 117 & 90 & 252 & 200 & 107 & 528 & 461 & 216 & 11.8 & 11.1 & 7.1 \\
\hline 28 & 28 & 28 & 28 & 28 & 28 & 28 & 28 & 28 & 0.2 & 0.2 & 0.2 \\
\hline
\end{tabular}

\begin{tabular}{|c|c|c|c|c|c|c|c|c|c|c|c|}
\hline 51 & 51 & 51 & 53 & 53 & 52 & 55 & 55 & 54 & 0.7 & 0.7 & 0.7 \\
\hline 6 & 6 & 6 & 6 & 6 & 6 & 7 & 6 & 6 & -0.1 & -0.9 & -0.9 \\
\hline 206 & 206 & 204 & 219 & 220 & 218 & 229 & 231 & 229 & 1.4 & 1.5 & 1.4 \\
\hline 17 & 17 & 17 & 17 & 17 & 17 & 17 & 17 & 17 & 0.8 & 0.8 & 0.8 \\
\hline 47 & 47 & 47 & 50 & 50 & 50 & 54 & 54 & 53 & 1.5 & 1.4 & 1.4 \\
\hline 3 & 3 & 3 & 4 & 4 & 4 & 4 & 4 & 4 & 1.0 & 1.0 & 0.9 \\
\hline 329 & 329 & 328 & 349 & 349 & 347 & 366 & 366 & 363 & 1.2 & 1.2 & 1.2 \\
\hline 168 & 168 & 168 & 172 & 172 & 172 & 176 & 176 & 176 & 0.7 & 0.7 & 0.7 \\
\hline 161 & 161 & 160 & 177 & 177 & 175 & 190 & 190 & 188 & 1.8 & 1.8 & 1.8 \\
\hline 28 & 28 & 28 & 30 & 30 & 30 & 25 & 25 & 26 & -2.7 & -2.7 & -2.6 \\
\hline 1192 & 1172 & 1101 & 1328 & 1282 & 1177 & 1493 & 1419 & 1287 & 1.9 & 1.7 & 1.2 \\
\hline 1050 & 1039 & 924 & 1123 & 1105 & 956 & 1212 & 1189 & 1003 & 1.3 & 1.2 & 0.4 \\
\hline 1147 & 1131 & 1065 & 1213 & 1184 & 1075 & 1279 & 1240 & 1082 & 1.2 & 1.1 & 0.4 \\
\hline 21 & 20 & 18 & 35 & 32 & 28 & 45 & 40 & 34 & 10.1 & 9.5 & 8.7 \\
\hline 3411 & 3363 & 3107 & 3699 & 3604 & 3236 & 4029 & 3889 & 3407 & 1.5 & 1.4 & 0.7 \\
\hline
\end{tabular}

\begin{tabular}{|c|c|c|c|c|c|c|c|c|c|c|c|}
\hline 8.6 & 8.4 & 8.2 & 8.8 & 8.4 & 8.1 & 9.2 & 8.4 & 7.9 & 0.4 & 0.0 & -0.3 \\
\hline 7.8 & 7.7 & 7.6 & 7.9 & 7.6 & 7.4 & 8.2 & 7.6 & 7.2 & 0.1 & -0.2 & -0.5 \\
\hline 5.0 & 4.9 & 4.8 & 5.0 & 4.8 & 4.6 & 5.1 & 4.8 & 4.5 & 0.1 & -0.2 & -0.6 \\
\hline 5.3 & 5.3 & 5.4 & 5.3 & 5.3 & 5.3 & 5.3 & 5.3 & 5.2 & 0.2 & 0.2 & 0.1 \\
\hline 7.1 & 7.0 & 6.9 & 7.3 & 7.0 & 6.7 & 7.6 & 7.0 & 6.6 & 0.3 & -0.1 & -0.4 \\
\hline
\end{tabular}

\begin{tabular}{|c|c|c|c|c|c|c|c|c|c|c|c|}
\hline 2.5 & 2.5 & 2.6 & 2.5 & 2.4 & 2.5 & 2.5 & 2.4 & 2.4 & -0.4 & -0.6 & -0.7 \\
\hline 1.3 & 1.2 & 1.0 & 1.4 & 1.2 & 1.0 & 1.5 & 1.2 & 1.1 & 1.1 & 0.1 & -0.5 \\
\hline 2.8 & 2.8 & 2.9 & 2.8 & 2.8 & 2.9 & 2.7 & 2.7 & 2.7 & -0.1 & -0.2 & -0.2 \\
\hline 0.4 & 0.4 & 0.4 & 0.6 & 0.5 & 0.4 & 0.8 & 0.6 & 0.4 & 3.8 & 2.5 & 0.6 \\
\hline 7.1 & 7.0 & 6.9 & 7.3 & 7.0 & 6.7 & 7.6 & 7.0 & 6.6 & 0.3 & -0.1 & -0.4 \\
\hline
\end{tabular}

Note: Totals may not equal sum of components due to independent rounding.

Sources: 1993 and 1994 commercial and transportation sales: Total transportation plus commercial sales come from Energy Information Administration (ElA), State Energy Data Report 1993, DOE/EIA-0214(93) (Washington, DC, August 1995), but individual sectors do not match because sales taken from commercial and placed in transportation, according to Oak Ridge National Laboratories, Transportation Energy Data Book 15 (May 1995) which indicates the transportation value should be higher. 1993 and 1994 generation by electric utilities, nonutilities, and cogenerators, net electricity imports, residential sales, and industrial sales: EIA, Annual Energy Review 1994, DOE/EIA-0384(94) (Washington, DC, July 1995). 1993 residential electric prices derived from EIA, Short Term Energy Outlook, DOE/EIA-0202(95/3Q) (Washington, DC, August 1995). 1993 and 1994 electricity prices for commercial, industrial, and transportation; price components; and projections: ElA, AEO96 National Energy Modeling System runs TECHO1.D031396A (Slow Technology), AEO96B.D101995C (reference), and TECH04.D031396B (Rapid Technology). 


\begin{tabular}{|c|c|c|c|c|c|c|}
\hline \multirow[b]{3}{*}{ Net Summer Capability ${ }^{1}$} & \multirow[b]{3}{*}{1993} & \multirow[b]{3}{*}{1994} & \multirow[b]{3}{*}{1995} & \multicolumn{3}{|c|}{ Projections } \\
\hline & & & & \multicolumn{3}{|c|}{2000} \\
\hline & & & & $\begin{array}{c}\text { Slow } \\
\text { Technology }\end{array}$ & Reference & $\begin{array}{c}\text { Rapid } \\
\text { Technology }\end{array}$ \\
\hline
\end{tabular}

Electric Utilities

\section{Capability}

Coal Steam . .

Other Fossil Steam ${ }^{2} \ldots \ldots \ldots \ldots \ldots \ldots \ldots \ldots \ldots \ldots$

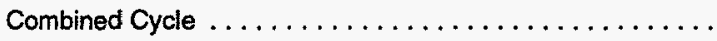

Combustion Turbine/Diesel . ...................

Nuclear Power .............................

Pumped Storage . . . . . . . . . . . . . . . . . . . . .

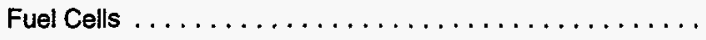

Renewable Sources ${ }^{3} \ldots \ldots \ldots \ldots \ldots \ldots \ldots \ldots \ldots \ldots$

Total

Cumulative Planned Additions

Coal Steam . . . . . . . . . .

Other Fossil Steam ${ }^{2} \ldots \ldots \ldots \ldots \ldots \ldots \ldots \ldots \ldots \ldots$

Combined Cycle . . . . . . . . . . . . . . . . . .

Combustion Turbine/Diesel . . . . . . . . . . . . . . .

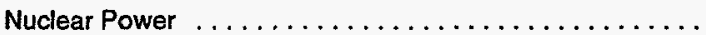

Pumped Storage . . . . . . . . . . . . . . . . . . .

Fuel Cells . . . . . . . . . . . . . . . . . . . .

Renewable Sources ${ }^{3} \ldots \ldots \ldots \ldots \ldots \ldots \ldots \ldots \ldots$

Total

$\begin{array}{rrr}302.4 & 301.9 & 303.0 \\ 141.0 & 140.0 & 138.3 \\ 11.4 & 11.5 & 12.7 \\ 50.8 & 51.0 & 54.3 \\ 99.1 & 99.1 & 99.2 \\ 19.0 & 19.0 & 19.9 \\ 0.0 & 0.0 & 0.0 \\ 77.2 & 77.2 & 77.5 \\ 700.9 & 699.8 & 704.8\end{array}$

298.0
128.0
17.9
76.4
100.3
19.9
0.6
78.5
719.5

Cumulative Unplanned Additions ${ }^{4}$

Coal Steam . ............................ 0

Other Fossil Steam ${ }^{2} \ldots \ldots \ldots \ldots \ldots \ldots \ldots \ldots \ldots \ldots \ldots, \quad 0.0$

Combined Cycle $\ldots \ldots \ldots \ldots \ldots \ldots \ldots \ldots \ldots \ldots \ldots \ldots . \ldots \ldots \ldots$

Combustion Turbine/Diesel . . . . . . . . . . . . . $\quad 0.0$

Nuclear Power ........................... 0.0

Pumped Storage $\ldots \ldots \ldots \ldots \ldots \ldots \ldots \ldots \ldots \ldots \ldots \ldots, \quad 0.0$

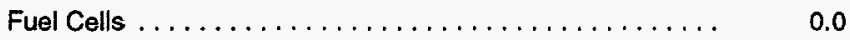

Renewable Sources ${ }^{3} \ldots \ldots \ldots \ldots \ldots \ldots \ldots \ldots \ldots$

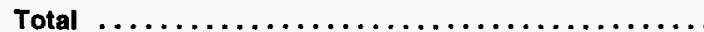

Cumulative Total Additions

Cumulative Retirements 5

\section{5}

\section{8}

Nonutilities (excludes cogenerators) ${ }^{6,7}$

\section{Capability}

Coal Steam . . . . . . . . . . . . . . . . . . . . . .

Other Fossil Steam ${ }^{2} \ldots \ldots \ldots \ldots \ldots \ldots \ldots \ldots \ldots \ldots \ldots$

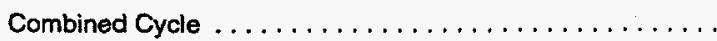

Combustion Turbine/Diesel . . . . . . . . . . . . . . . .

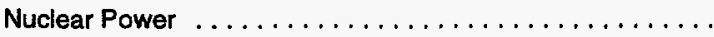

Pumped Storage . . . . . . . . . . . . . . . . . .

Fuel Cells . . . . . . . . . . . . . . . . . . . .

Renewable Sources ${ }^{3} \ldots \ldots \ldots \ldots \ldots \ldots \ldots \ldots \ldots \ldots \ldots$

Total $\ldots \ldots \ldots \ldots \ldots \ldots \ldots \ldots \ldots \ldots$

$\begin{array}{rrr}2.4 & 2.4 & 2.6 \\ 1.0 & 1.0 & 1.1 \\ 2.0 & 2.0 & 2.0 \\ 1.9 & 1.9 & 1.9 \\ 0.0 & 0.0 & 0.0 \\ 0.0 & 0.0 & 0.0 \\ 0.0 & 0.0 & 0.0 \\ 10.8 & 10.9 & 11.7 \\ 18.1 & 18.2 & 19.4\end{array}$

298.0

128.0

19.2

75.9

100.3

19.9

0.3

78.5

720.1

\section{1}

0.4

6.1

15.9

1.2

1.1

0.0

0.8

29.6

297.7

128.0

17.8

76.1

100.3

19.9

0.0

78.8

718.6

$\begin{array}{rr}4.1 & 4.1 \\ 0.4 & 0.4 \\ 6.1 & 6.1 \\ 15.9 & 15.9 \\ 1.2 & 1.2 \\ 1.1 & 1.1 \\ 0.0 & 0.0 \\ 0.8 & 0.8 \\ 29.6 & 29.6\end{array}$

0.3

0.0

0.3

9.9

0.0

0.0

0.6

0.1

11.2

40.8

30.9

41.3

30.9

0.0

0.0

0.3

9.6

0.0

0.0

0.0

0.4

10.3

39.8

30.9 
Table B9. Electricity Generating Capability (Continued) (Thousand Megawatts)

\begin{tabular}{|c|c|c|c|c|c|c|c|c|c|c|c|}
\hline \multicolumn{9}{|c|}{ Projections } & \multirow{2}{*}{\multicolumn{3}{|c|}{$\begin{array}{c}\text { Annual Growth } \\
\text { 1994-2015 (percent) }\end{array}$}} \\
\hline \multicolumn{3}{|c|}{2005} & \multicolumn{3}{|c|}{2010} & \multicolumn{3}{|c|}{2015} & & & \\
\hline $\begin{array}{c}\text { Slow } \\
\text { Technology }\end{array}$ & Reference & $\begin{array}{c}\text { Rapid } \\
\text { Technology }\end{array}$ & $\begin{array}{c}\text { Slow } \\
\text { Technology }\end{array}$ & Reference & $\begin{array}{c}\text { Rapid } \\
\text { Technology }\end{array}$ & $\begin{array}{c}\text { Slow } \\
\text { Technology }\end{array}$ & Reference & $\mid \begin{array}{c}\text { Rapid } \\
\text { Technology }\end{array}$ & $\begin{array}{c}\text { Slow } \\
\text { Technology }\end{array}$ & Reference & $\begin{array}{c}\text { Rapid } \\
\text { Technology }\end{array}$ \\
\hline
\end{tabular}

\begin{tabular}{|c|c|c|c|c|c|c|c|c|c|c|c|}
\hline 304.0 & 302.1 & 301.7 & 309.7 & 308.8 & 305.8 & 323.1 & 312.6 & 305.2 & 0.3 & 0.2 & 0.1 \\
\hline 121.8 & 121.8 & 121.8 & 118.2 & 118.2 & 118.2 & 113.5 & 113.5 & 113.5 & -1.0 & -1.0 & -1.0 \\
\hline 26.6 & 27.1 & 25.2 & 36.9 & 33.4 & 25.7 & 63.1 & 56.4 & 33.0 & 8.4 & 7.9 & 5.1 \\
\hline 93.3 & 90.4 & 87.2 & 107.1 & 103.4 & 90.2 & 121.4 & 115.8 & 100.7 & 4.2 & 4.0 & 3.3 \\
\hline 99.7 & 99.7 & 99.7 & 93.3 & 93.3 & 93.3 & 63.6 & 63.6 & 63.6 & -2.1 & -2.1 & -2.1 \\
\hline 19.9 & 19.9 & 19.9 & 19.9 & 19.9 & 19.9 & 19.9 & 19.9 & 19.9 & 0.2 & 0.2 & 0.2 \\
\hline 0.8 & 0.5 & 0.0 & 1.1 & 0.6 & 0.2 & 2.7 & 1.4 & 1.5 & 52.0 & 47.3 & 47.8 \\
\hline 79.0 & 79.0 & 79.1 & 79.4 & 79.6 & 79.3 & 81.7 & 82.0 & 80.4 & 0.3 & 0.3 & 0.2 \\
\hline 745.0 & 740.5 & 734.6 & 765.6 & 757.2 & 732.5 & 788.9 & 765.0 & 717.7 & 0.6 & 0.4 & 0.1 \\
\hline 9.1 & 9.1 & 9.1 & 14.8 & 14.8 & 14.8 & 16.5 & 16.5 & 16.5 & 29.7 & 29.7 & 29.7 \\
\hline 0.4 & 0.4 & 0.4 & 0.4 & 0.4 & 0.4 & 0.4 & 0.4 & 0.4 & N/A & N/A & N/A \\
\hline 13.4 & 13.4 & 13.4 & 13.6 & 13.6 & 13.6 & 13.6 & 13.6 & 13.6 & 24.5 & 24.5 & 24.5 \\
\hline 27.6 & 27.6 & 27.6 & 28.5 & 28.5 & 28.5 & 28.5 & 28.5 & 28.5 & 24.5 & 24.5 & 24.5 \\
\hline 1.2 & 1.2 & 1.2 & 1.2 & 1.2 & 1.2 & 1.2 & 1.2 & 1.2 & $N / A$ & N/A & N/A \\
\hline 1.1 & 1.1 & 1.1 & 1.1 & 1.1 & 1.1 & 1.1 & 1.1 & 1.1 & 6.6 & 6.6 & 6.6 \\
\hline 0.0 & 0.0 & 0.0 & 0.0 & 0.0 & 0.0 & 0.0 & 0.0 & 0.0 & N/A & N/A & N/A \\
\hline 1.1 & 1.1 & 1.1 & 1.1 & 1.1 & 1.1 & 1.1 & 1.1 & 1.1 & 36.2 & 36.2 & 36.2 \\
\hline 53.8 & 53.8 & 53.8 & 60.6 & 60.6 & 60.6 & 62.4 & 62.4 & 62.4 & 23.1 & 23.1 & 23.1 \\
\hline
\end{tabular}

\begin{tabular}{|c|c|c|c|c|c|c|c|c|c|c|c|}
\hline 1.4 & 0.9 & 0.5 & 2.0 & 1.7 & 0.6 & 15.8 & 5.9 & 1.1 & N/A & N/A & N/A \\
\hline 0.0 & 0.0 & 0.0 & 0.0 & 0.0 & 0.0 & 0.0 & 0.0 & 0.0 & N/A & N/A & N/A \\
\hline 1.7 & 2.2 & 0.3 & 11.9 & 8.3 & 0.6 & 38.0 & 31.3 & 7.9 & $\mathrm{~N} / \mathrm{A}$ & N/A & $N / A$ \\
\hline 15.8 & 12.8 & 9.6 & 28.9 & 25.3 & 12.0 & 44.4 & 38.7 & 23.7 & N/A & N/A & N/A \\
\hline 0.0 & 0.0 & 0.0 & 0.0 & 0.0 & 0.0 & 0.0 & 0.0 & 0.0 & N/A & N/A & N/A \\
\hline 0.0 & 0.0 & 0.0 & 0.0 & 0.0 & 0.0 & 0.0 & 0.0 & 0.0 & N/A & $N / A$ & N/A \\
\hline 0.8 & 0.5 & 0.0 & 1.1 & 0.6 & 0.2 & 2.7 & 1.4 & 1.5 & $N / A$ & $\mathrm{~N} / \mathrm{A}$ & $\mathrm{N} / \mathrm{A}$ \\
\hline 0.4 & 0.4 & 0.6 & 1.0 & 1.1 & 0.9 & 3.6 & 3.9 & 2.3 & N/A & $\mathrm{N} / \mathrm{A}$ & N/A \\
\hline 20.1 & 16.8 & 11.0 & 44.8 & 37.0 & 14.3 & 104.4 & 81.1 & 36.4 & NA & NA & NA \\
\hline 74.0 & 70.7 & 64.8 & 105.5 & 97.6 & 74.9 & 166.7 & 143.5 & 98.8 & 29.0 & 28.1 & 25.8 \\
\hline 40.3 & 40.3 & 40.3 & 53.9 & 53.9 & 53.9 & 93.9 & 93.9 & 93.9 & 11.2 & 11.2 & 11.2 \\
\hline
\end{tabular}

$\begin{array}{rrrrrrrrrrrr}4.1 & 3.5 & 3.4 & 5.7 & 4.4 & 3.7 & 13.5 & 6.5 & 4.1 & 8.6 & 4.9 & 2.6 \\ 1.1 & 1.1 & 1.1 & 1.1 & 1.1 & 1.1 & 1.1 & 1.1 & 1.1 & 0.3 & 0.3 & 0.3 \\ 5.7 & 4.8 & 3.3 & 17.0 & 11.8 & 3.5 & 45.8 & 37.6 & 10.2 & 16.1 & 15.0 & 8.1 \\ 19.0 & 15.5 & 10.8 & 40.1 & 31.5 & 13.8 & 55.1 & 43.9 & 29.8 & 17.5 & 16.2 & 14.1 \\ 0.0 & 0.0 & 0.0 & 0.0 & 0.0 & 0.0 & 0.0 & 0.0 & 0.0 & \text { N/A } & \text { N/A } & \text { N/A } \\ 0.0 & 0.0 & 0.0 & 0.0 & 0.0 & 0.0 & 0.0 & 0.0 & 0.0 & \text { N/A } & \text { N/A } & \text { N/A } \\ 0.1 & 0.1 & 0.0 & 0.1 & 0.2 & 0.1 & 0.8 & 1.0 & 0.4 & \text { N/A } & \text { N/A } & \text { N/A } \\ 15.1 & 15.5 & 15.3 & 16.5 & 17.5 & 16.7 & 22.0 & 24.4 & 20.6 & 3.4 & 3.9 & 3.1 \\ 45.1 & 40.5 & 34.0 & 80.5 & 66.5 & 38.8 & 138.2 & 114.5 & 66.2 & 10.1 & 9.2 & 6.3\end{array}$


Table B9. Electricity Generating Capability (Continued) (Thousand Megawatts)

\begin{tabular}{|c|c|c|c|c|c|c|}
\hline \multirow[b]{3}{*}{ Net Summer Capability ${ }^{1}$} & \multirow[b]{3}{*}{1993} & \multirow[b]{3}{*}{1994} & \multirow[b]{3}{*}{1995} & \multicolumn{3}{|c|}{ Projections } \\
\hline & & & & \multicolumn{3}{|c|}{2000} \\
\hline & & & & $\begin{array}{c}\text { Slow } \\
\text { Technology }\end{array}$ & Reference & $\begin{array}{c}\text { Rapid: } \\
\text { Technology }\end{array}$ \\
\hline Cogenerators ${ }^{7}$ & & & & & & \\
\hline Capacity & & & & & & \\
\hline$\ldots \ldots \ldots \ldots \ldots \ldots \ldots \ldots \ldots \ldots$ & 8.0 & 7.7 & 8.2 & 9.2 & 9.2 & 9.2 \\
\hline Petroleum . . . . . . . . . . . . . . . . . . & 0.8 & 0.8 & 0.9 & 0.9 & 0.9 & 0.9 \\
\hline 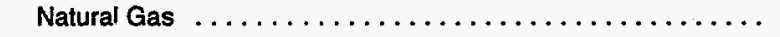 & 27.4 & 28.7 & 29.5 & 31.9 & 31.9 & 31.8 \\
\hline Other Gaseous Fuels $\ldots \ldots \ldots \ldots \ldots \ldots \ldots$ & 0.9 & 0.9 & 0.9 & 1.0 & 1.0 & 1.0 \\
\hline Renewable Sources ${ }^{3} \ldots \ldots \ldots \ldots \ldots \ldots \ldots \ldots \ldots$ & 5.9 & 6.1 & 6.2 & 6.6 & 6.6 & 6.6 \\
\hline 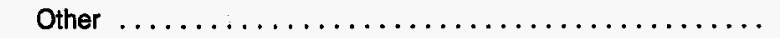 & 0.1 & 0.0 & 0.0 & 0.0 & 0.0 & 0.0 \\
\hline Total $\ldots \ldots \ldots \ldots \ldots \ldots \ldots \ldots \ldots \ldots \ldots \ldots$ & 43.1 & 44.3 & 45.6 & 49.5 & 49.5 & 49.5 \\
\hline Cumulative Additions ${ }^{4} \ldots \ldots \ldots \ldots \ldots \ldots \ldots \ldots \ldots \ldots$ & 8.4 & 9.7 & 12.1 & 33.0 & 31.2 & 29.6 \\
\hline
\end{tabular}


Table B9. Electricity Generating Capability (Continued) (Thousand Megawatts)

\begin{tabular}{|c|c|c|c|c|c|c|c|c|c|c|c|}
\hline \multicolumn{9}{|c|}{ Projections } & \multirow{2}{*}{\multicolumn{3}{|c|}{$\begin{array}{c}\text { Annual Growth } \\
\text { 1994-2015 (percent) }\end{array}$}} \\
\hline \multicolumn{3}{|c|}{2005} & \multicolumn{3}{|c|}{2010} & \multicolumn{3}{|c|}{2015} & & & \\
\hline $\begin{array}{c}\text { Slow } \\
\text { Technology } \\
\end{array}$ & Relerence & $\begin{array}{c}\text { Rapid } \\
\text { Technology }\end{array}$ & $\begin{array}{c}\text { Slow } \\
\text { Technology }\end{array}$ & Reference & $\begin{array}{c}\text { Rapid } \\
\text { Technology }\end{array}$ & $\begin{array}{c}\text { Slow } \\
\text { Technology }\end{array}$ & Reference & $\begin{array}{c}\text { Rapid } \\
\text { Technology }\end{array}$ & $\begin{array}{c}\text { Slow } \\
\text { Technology }\end{array}$ & Reference & $\begin{array}{c}\text { Aapid } \\
\text { Technology }\end{array}$ \\
\hline 9.6 & 9.6 & 9.6 & 9.9 & 9.9 & 9.9 & 10.3 & 10.2 & 10.2 & 1.4 & 1.4 & 1.3 \\
\hline 1.0 & 1.0 & 1.0 & 1.0 & 1.0 & 1.0 & 1.0 & 1.0 & 1.0 & 1.0 & 1.0 & 0.9 \\
\hline 33.9 & 33.9 & 33.7 & 35.7 & 35.8 & 35.5 & 37.2 & 37.2 & 37.0 & 1.2 & 1.2 & 1.2 \\
\hline 1.0 & 1.0 & 1.0 & 1.0 & 1.0 & 1.0 & 1.0 & 1.0 & 1.0 & 0.1 & 0.1 & 0.1 \\
\hline 7.2 & 7.2 & 7.2 & 7.7 & 7.7 & 7.7 & 8.2 & 8.2 & 8.1 & 1.4 & 1.4 & 1.4 \\
\hline 0.0 & 0.0 & 0.0 & 0.0 & 0.0 & 0.0 & 0.0 & 0.0 & 0.0 & -0.3 & -0.3 & -0.3 \\
\hline 52.6 & 52.6 & 52.4 & 55.3 & 55.3 & 55.0 & 57.7 & 57.7 & 57.3 & 1.3 & 1.3 & 1.2 \\
\hline 44.9 & 40.3 & 33.6 & 82.9 & 69.0 & 41.0 & 143.1 & 119.3 & 70.7 & 13.7 & 12.7 & 9.9 \\
\hline
\end{tabular}

'Net summer capability is the steady hourly output that generating equipment is expected to supply to system load (exclusive of auxiliary power), as demonstrated by tests during summer peak demand.

Includes oil-, gas-, and dual-fired capability.

Includes conventional hydroelectric, geothermal, wood, wood waste, municipal solid waste, other biomass, solar and wind power.

Cumulative additions after December 31, 1993. Non-zero utility planned additions in 1993 indicate units operational in 1993, but not supplying power to the grid.

SCumulative total retirements from 1993.

Inctudes nonutilities except for cogenerators. These facilities include small power producers, exempt wholesale generators and generators at industrial and commercial facilities which provide electricity for on-site use and for sale to utilities.

'Nameplate capacity is reported for nonutilites on Form ElA-867, "Annual Power Producer Report." Nameplate capacity is designated by the manufacturer. The nameplate capacity has been converted to the net summer capacity based on historic relationships.

N/A $=$ Not applicable.

Notes: Totals may not equal sum of components due to independent rounding. Net summer capacity has been estimated for nonutility generators for AEO96. Net summer capacity is used to be consistent with electric utility capacity estimates. Electric utility capacity is the most recent data available as of August 15, 1994. Therefore, capacity estimates may differ from other Energy Information Administration sources.

Sources: 1993 and 1994 net summer capacity at electric utilities and planned additions: Energy Information Administration (EIA), Form ElA-860, "Annual Electric Generator Report." Net summer capacity for nonutilities and cogeneration in 1993 and 1994 and planned additions estimated based on EIA, Form EIA-867, “Annual Nonutility Power Producer Report." Projections: EIA, AEO96 National Energy Modeling System runs TECH01.D031396A (Slow Technology), AEO96B.D101995C (reference), and TECH04.D031396B (Rapid Technology). 
Table B10. Petroleum Supply and Disposition Balance (Million Barrels per Day, Unless Otherwise Noted)

\begin{tabular}{|c|c|c|c|c|c|c|}
\hline \multirow[b]{3}{*}{ Supply and Disposition } & \multirow[b]{3}{*}{1993} & \multirow[b]{3}{*}{1994} & \multirow[b]{3}{*}{1995} & \multicolumn{3}{|c|}{ Projections } \\
\hline & & & & \multicolumn{3}{|c|}{2000} \\
\hline & & & & $\begin{array}{c}\text { Slow } \\
\text { Technology } \\
\end{array}$ & Reference & $\begin{array}{c}\text { High } \\
\text { Technology }\end{array}$ \\
\hline \multicolumn{7}{|l|}{ Crude Oil } \\
\hline Domestic Crude Production' & 6.85 & 6.66 & 6.54 & 5.52 & 5.65 & 5.79 \\
\hline Alaska $\ldots \ldots \ldots \ldots \ldots \ldots \ldots \ldots \ldots \ldots$ & 1.58 & 1.56 & 1.49 & 1.11 & 1.16 & 1.21 \\
\hline Lower 48 States . . . . . . . . . . . . . . . . . . . . . . & 5.26 & 5.10 & 5.05 & 4.41 & 4.49 & 4.57 \\
\hline Net Imports $\ldots \ldots \ldots \ldots \ldots \ldots \ldots \ldots \ldots \ldots \ldots$ & 6.67 & 6.95 & 7.40 & 9.06 & 8.95 & 8.62 \\
\hline Other Crude Supply ${ }^{2} \ldots \ldots \ldots \ldots \ldots \ldots \ldots \ldots$ & 0.08 & 0.24 & 0.00 & 0.00 & 0.00 & 0.00 \\
\hline Total Crude Supply $\ldots \ldots \ldots \ldots \ldots \ldots \ldots$ & 13.60 & 13.85 & 13.94 & 14.58 & 14.60 & 14.41 \\
\hline Natural Gas Plant Liquids $\ldots \ldots \ldots \ldots \ldots \ldots \ldots$ & 1.74 & 1.73 & 1.80 & 1.88 & 1.85 & 1.72 \\
\hline Other Inputs ${ }^{3} \ldots \ldots \ldots \ldots \ldots \ldots \ldots \ldots \ldots$ & 0.19 & 0.27 & 0.23 & 0.27 & 0.27 & 0.27 \\
\hline Refinery Processing Gain ${ }^{4} \ldots \ldots \ldots \ldots \ldots \ldots \ldots$ & 0.77 & 0.77 & 0.81 & 0.81 & 0.82 & 0.80 \\
\hline Net Product Imports ${ }^{5} \ldots \ldots \ldots \ldots \ldots \ldots \ldots \ldots \ldots$ & 0.93 & 1.09 & 0.85 & 1.24 & 1.13 & 0.94 \\
\hline Total Primary Supply ${ }^{6} \ldots \ldots \ldots \ldots \ldots \ldots$ & 17.22 & 17.70 & 17.64 & 18.79 & 18.66 & 18.15 \\
\hline \multicolumn{7}{|l|}{ Refined Petroleum Products Supplied } \\
\hline Motor Gasoline ${ }^{7} \ldots \ldots \ldots \ldots \ldots \ldots \ldots \ldots \ldots$ & 7.48 & 7.60 & 7.77 & 8.26 & 8.21 & 8.02 \\
\hline Jet Fuel ${ }^{8} \ldots \ldots \ldots \ldots \ldots \ldots \ldots \ldots \ldots \ldots \ldots \ldots \ldots \ldots$ & 1.47 & 1.53 & 1.58 & 1.80 & 1.80 & 1.79 \\
\hline Distillate Fuel $^{9} \ldots \ldots \ldots \ldots \ldots \ldots \ldots \ldots \ldots$ & 3.04 & 3.16 & 3.22 & 3.45 & 3.40 & 3.27 \\
\hline Residual Fuel $\ldots \ldots \ldots \ldots \ldots \ldots \ldots \ldots \ldots \ldots \ldots \ldots \ldots \ldots \ldots$ & 1.08 & 1.02 & 0.96 & 1.04 & 1.02 & 0.93 \\
\hline 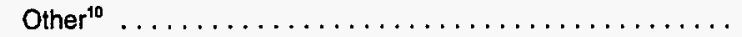 & 4.17 & 4.41 & 4.25 & 4.37 & 4.36 & 4.27 \\
\hline Total $\ldots \ldots \ldots \ldots \ldots \ldots \ldots \ldots \ldots \ldots$ & 17.24 & 17.72 & 17.78 & 18.91 & 18.78 & 18.28 \\
\hline \multicolumn{7}{|l|}{ Refined Petroleum Products Supplied } \\
\hline Residential and Commercial $\ldots \ldots \ldots \ldots \ldots \ldots \ldots$ & 1.14 & 1.17 & 1.08 & 1.10 & 1.08 & 1.09 \\
\hline Industrial| $^{11} \ldots \ldots \ldots \ldots \ldots \ldots \ldots \ldots \ldots \ldots$ & 4.45 & 4.58 & 4.66 & 4.83 & 4.81 & 4.68 \\
\hline Transportation $\ldots \ldots \ldots \ldots \ldots \ldots \ldots \ldots \ldots$ & 11.18 & 11.50 & 11.72 & 12.68 & 12.59 & 12.28 \\
\hline Electric Generators ${ }^{12}$. & 0.46 & 0.47 & 0.33 & 0.31 & 0.30 & 0.22 \\
\hline Total $\ldots \ldots \ldots \ldots \ldots \ldots \ldots \ldots \ldots \ldots \ldots \ldots$ & 17.24 & 17.72 & 17.78 & 18.91 & 18.78 & 18.28 \\
\hline 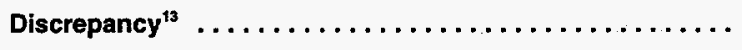 & -0.02 & -0.01 & -0.14 & -0.13 & -0.12 & -0.13 \\
\hline World Oil Price (1994 dollars per barrel) ${ }^{14} \ldots \ldots \ldots \ldots \ldots$. . . & 16.48 & 15.52 & 16.81 & 19.66 & 19.27 & 18.48 \\
\hline Import Share of Product Supplied . . . . . . . . . . . . & 0.44 & 0.45 & 0.46 & 0.54 & 0.54 & 0.52 \\
\hline Domestic Refinery Distillation Capacity . . . . . . . . . . . & 15.1 & 15.0 & 15.4 & 15.7 & 15.6 & 15.6 \\
\hline Capacity Utilization Rate (percent) . . . . . . . . . . . & 92.0 & 93.0 & 90.7 & 93.3 & 93.4 & 92.6 \\
\hline
\end{tabular}


Table B10. Petroleum Supply and Disposition Balance (Continued) (Million Barrels per Day, Unless Otherwise Noted)

\begin{tabular}{|c|c|c|c|c|c|c|c|c|c|c|c|}
\hline \multicolumn{9}{|c|}{ Projections } & \multirow{2}{*}{\multicolumn{3}{|c|}{$\begin{array}{c}\text { Annual Growth } \\
1994-2015 \text { (percent) }\end{array}$}} \\
\hline \multicolumn{3}{|c|}{2005} & \multicolumn{3}{|c|}{2010} & \multicolumn{3}{|c|}{2015} & & & \\
\hline $\begin{array}{c}\text { Slow } \\
\text { Technology }\end{array}$ & Reference & $\begin{array}{c}\text { Rapid } \\
\text { Technology }\end{array}$ & $\begin{array}{c}\text { Slow } \\
\text { Technology }\end{array}$ & Reference & $\begin{array}{c}\text { Rapid } \\
\text { Technology }\end{array}$ & $\begin{array}{c}\text { Slow } \\
\text { Technology }\end{array}$ & Reference & $\begin{array}{c}\text { Rapid } \\
\text { Technology }\end{array}$ & $\begin{array}{c}\text { Slow } \\
\text { Technology }\end{array}$ & Reference & $\begin{array}{c}\text { Papid } \\
\text { Technology }\end{array}$ \\
\hline 5.02 & 5.25 & 5.49 & 5.14 & 5.44 & 5.59 & 5.39 & 5.81 & 6.04 & -1.0 & -0.6 & -0.5 \\
\hline 0.73 & 0.80 & 0.92 & 0.63 & 0.71 & 0.78 & 0.65 & 0.73 & 0.80 & -4.1 & -3.5 & -3.1 \\
\hline 4.29 & 4.44 & 4.56 & 4.51 & 4.73 & 4.81 & 4.74 & 5.08 & 5.24 & -0.4 & 0.0 & 0.1 \\
\hline 10.16 & 9.62 & 8.83 & 10.58 & 9.58 & 8.69 & 10.43 & 9.42 & 8.19 & 2.0 & 1.5 & 0.8 \\
\hline 0.00 & 0.00 & 0.00 & 0.00 & 0.00 & 0.00 & 0.00 & 0.00 & 0.00 & N/A & N/A & N/A \\
\hline 15.18 & 14.86 & 14.32 & 15.72 & 15.02 & 14.28 & 15.82 & 15.23 & 14.23 & 0.6 & 0.5 & 0.1 \\
\hline 2.03 & 1.97 & 1.75 & 2.19 & 2.11 & 1.83 & 2.26 & 2.27 & 2.02 & 1.3 & 1.3 & 0.7 \\
\hline 0.31 & 0.31 & 0.30 & 0.32 & 0.31 & 0.30 & 0.32 & 0.31 & 0.29 & 0.8 & 0.7 & 0.4 \\
\hline 0.85 & 0.85 & 0.82 & 0.88 & 0.87 & 0.83 & 0.90 & 0.86 & 0.78 & 0.7 & 0.5 & 0.1 \\
\hline 1.87 & 1.79 & 1.33 & 2.30 & 2.26 & 1.12 & 3.22 & 2.37 & 0.71 & 5.3 & 3.8 & -2.0 \\
\hline 20.24 & 19.78 & 18.52 & 21.41 & 20.56 & 18.36 & 22.51 & 21.04 & 18.04 & 1.2 & 0.8 & 0.1 \\
\hline 8.81 & 8.53 & 8.15 & 9.19 & 8.64 & 8.01 & 9.40 & 8.56 & 7.71 & 1.0 & 0.6 & 0.1 \\
\hline 2.02 & 1.99 & 1.96 & 2.20 & 2.14 & 2.06 & 2.36 & 2.27 & 2.13 & 2.1 & 1.9 & 1.6 \\
\hline 3.71 & 3.64 & 3.31 & 3.95 & 3.86 & 3.31 & 4.31 & 4.11 & 3.35 & 1.5 & 1.3 & 0.3 \\
\hline 1.24 & 1.18 & 1.03 & 1.40 & 1.33 & 1.12 & 1.57 & 1.36 & 1.26 & 2.1 & 1.4 & 1.0 \\
\hline 4.58 & 4.54 & 4.18 & 4.79 & 4.69 & 3.96 & 5.04 & 4.89 & 3.73 & 0.6 & 0.5 & -0.8 \\
\hline 20.36 & 19.89 & 18.63 & 21.53 & 20.67 & 18.46 & 22.68 & 21.18 & 18.18 & 1.2 & 0.9 & 0.1 \\
\hline 1.07 & 1.05 & 1.05 & 1.05 & 1.02 & 1.02 & 1.06 & 1.01 & 0.99 & -0.5 & -0.7 & -0.8 \\
\hline 5.09 & 5.03 & 4.58 & 5.31 & 5.18 & 4.25 & 5.63 & 5.38 & 3.95 & 1.0 & 0.8 & -0.7 \\
\hline 13.74 & 13.39 & 12.73 & 14.60 & 13.93 & 12.85 & 15.29 & 14.29 & 12.79 & 1.4 & 1.0 & 0.5 \\
\hline 0.46 & 0.42 & 0.26 & 0.57 & 0.52 & 0.34 & 0.70 & 0.50 & 0.45 & 1.9 & 0.3 & -0.3 \\
\hline 20.36 & 19.89 & 18.63 & 21.53 & 20.67 & 18.46 & 22.68 & 21.18 & 18.18 & 1.2 & 0.9 & 0.1 \\
\hline-0.12 & -0.11 & -0.11 & -0.12 & -0.10 & -0.10 & -0.16 & -0.14 & -0.14 & N/A & N/A & $\mathbf{N} / \mathbf{A}$ \\
\hline 22.99 & 21.86 & 19.77 & 25.59 & 23.70 & 20.55 & 28.58 & 25.43 & 21.17 & 3.0 & 2.4 & 1.5 \\
\hline 0.59 & 0.57 & 0.55 & 0.60 & 0.57 & 0.53 & 0.60 & 0.56 & 0.49 & 1.4 & 1.0 & 0.4 \\
\hline 16.5 & 16.1 & 15.7 & 16.9 & 16.2 & 15.7 & 17.0 & 16.4 & 15.7 & 0.6 & 0.4 & 0.2 \\
\hline 92.4 & 92.5 & 91.6 & 93.9 & 92.9 & 91.2 & 93.7 & 93.5 & 90.9 & 0.0 & 0.0 & -0.1 \\
\hline
\end{tabular}

'Includes lease condensate.

${ }^{2}$ Strategic petroleum supply stock additions plus unaccounted for crude oil plus crude stock withdrawals minus crude products supplied.

'Includes alcohols, ethers, petroleum product stock withdrawals, domestic sources of blending components, and other hydrocarbons.

'Represents volumetric gain in refinery distillation and cracking processes.

Includes net imports of finished petroleum products, unfinished oils, other hydrocarbons, alcohols, ethers, and blending components.

Total crude supply plus natural gas plant liquids plus other inputs plus refinery processing gain plus net petroleum imports.

Includes ethanol and ethers blended into gasoline.

Includes naphtha and kerosene type.

Includes distillate and kerosene.

10includes aviation gasoline, liquefied petroleum gas, petrochemical feedstocks, lubricants, waxes, asphalt, road oil, still gas, special naphthas, petroleum coke, crude oil product supplied, and miscellaneous petroleum products.

"Includes consumption by cogenerators.

${ }^{12}$ Includes all electric power generators except cogenerators, which produce electricity as a by-product of other processes.

${ }^{23}$ Balancing item. Includes unaccounted for supply, losses and gains.

${ }^{14}$ Average refiner acquisition cost for imported crude oil.

N/A = Not applicable.

Note: Totals may not equal sum of components due to independent rounding.

Sources: 1993: Energy Information Administration (EIA), Petroleum Supply Annual 1993, DOE/EIA-0340(93) (Washington, DC, June 1994). 1994: EIA, Petroleum Supply Annual 1994, DOE/EIA-0340(94) (Washington, DC, May 1995). Projections: EIA, AEO96 National Energy Modeling System runs TECHO1.D031396A (Slow Technology). AEO96B.D101995C (reference), and TECH04.D031396B (Rapid Technology). 
Table B11. Petroleum Product Prices (1994 Cents per Gallon Unless Otherwise Noted)

\begin{tabular}{|c|c|c|c|c|c|c|}
\hline \multirow[b]{3}{*}{ Sector and Fuel } & \multirow[b]{3}{*}{1993} & \multirow[b]{3}{*}{1994} & \multirow[b]{3}{*}{1995} & \multicolumn{3}{|c|}{ Projections } \\
\hline & & & & \multicolumn{3}{|c|}{2000} \\
\hline & & & & $\begin{array}{c}\text { Slow } \\
\text { Technology }\end{array}$ & Reference & $\begin{array}{c}\text { Rapid } \\
\text { Technology }\end{array}$ \\
\hline World Oil Price (dollars per barrel) $\ldots \ldots \ldots \ldots \ldots \ldots$ & 16.48 & 15.52 & 16.81 & 19.66 & 19.27 & 18.48 \\
\hline \multicolumn{7}{|l|}{ Delivered Sector Product Prices } \\
\hline \multicolumn{7}{|l|}{ Residential } \\
\hline$\ldots \ldots \ldots \ldots \ldots \ldots \ldots \ldots \ldots \ldots \ldots$ & 92.7 & 87.8 & 94.6 & 105.2 & 103.7 & 101.2 \\
\hline Liqueffed Petroleum Gas . . . . . . . . . . . . . . . . . & 92.9 & 90.1 & 94.9 & 103.5 & 102.4 & 102.3 \\
\hline \multicolumn{7}{|l|}{ Commercial } \\
\hline Distillate Fuel $\ldots \ldots \ldots$ & 65.3 & 61.6 & 64.5 & 75.0 & 73.5 & 70.7 \\
\hline Residual Fuel $\ldots \ldots \ldots \ldots \ldots$ & 43.1 & 42.6 & 39.7 & 46.4 & 45.4 & 43.2 \\
\hline Residual Fuel (dollars per barrel) $\ldots \ldots \ldots \ldots \ldots \ldots \ldots$ & 18.11 & 17.89 & 16.67 & 19.51 & 19.08 & 18.16 \\
\hline \multicolumn{7}{|l|}{ Industrial' } \\
\hline Distillate Fuel $\ldots \ldots \ldots \ldots$ & 67.5 & 63.6 & 64.3 & 75.4 & 73.9 & 71.2 \\
\hline Liquefied Petroleum Gas $\ldots \ldots \ldots \ldots \ldots \ldots \ldots \ldots \ldots$ & 42.0 & 52.8 & 50.4 & 58.3 & 57.4 & 57.5 \\
\hline Residual Fuel $\ldots \ldots \ldots \ldots \ldots \ldots \ldots \ldots \ldots \ldots \ldots$ & 36.5 & 37.5 & 38.0 & 44.7 & 43.7 & 41.6 \\
\hline Residual Fuel (dollars per barrel) $\ldots \ldots \ldots \ldots \ldots \ldots \ldots$ & 15.33 & 15.74 & 15.96 & 18.76 & 18.35 & 17.47 \\
\hline \multicolumn{7}{|l|}{ Transportation } \\
\hline Distillate Fuel ${ }^{2} \ldots \ldots \ldots \ldots \ldots \ldots \ldots \ldots \ldots$ & 113.9 & 111.5 & 114.7 & 123.4 & 121.9 & 118.9 \\
\hline Jet Fuel $^{3} \ldots \ldots \ldots \ldots \ldots \ldots \ldots \ldots \ldots \ldots$ & 59.2 & 53.3 & 57.9 & 73.7 & 72.2 & 69.3 \\
\hline Motor Gasoline $\ldots \ldots \ldots \ldots \ldots \ldots \ldots \ldots \ldots$ & 113.9 & 111.6 & 120.1 & 132.3 & 130.5 & 126.8 \\
\hline Residual Fuel $\ldots \ldots \ldots \ldots \ldots \ldots \ldots \ldots \ldots \ldots$ & $\mathbf{3 1 . 5}$ & 30.2 & 34.4 & 41.3 & 40.3 & 38.3 \\
\hline Residual Fuel (dollars per barrel) $\ldots \ldots \ldots \ldots \ldots \ldots \ldots$ & 13.22 & 12.68 & 14.45 & 17.34 & 16.94 & 16.10 \\
\hline \multicolumn{7}{|l|}{ Electric Generators ${ }^{5}$} \\
\hline$\ldots \ldots \ldots \ldots \ldots \ldots \ldots \ldots$ & 58.7 & 54.9 & 58.3 & 70.2 & 68.7 & 66.3 \\
\hline Residual Fuel $\ldots \ldots \ldots \ldots \ldots \ldots \ldots \ldots \ldots \ldots \ldots$ & 39.6 & 39.3 & 41.8 & 46.6 & 45.6 & 43.6 \\
\hline Residual Fuel (dollars per barrel) $\ldots \ldots \ldots \ldots \ldots \ldots \ldots$ & 16.62 & 16.49 & 17.54 & 19.56 & 19.14 & 18.29 \\
\hline \multicolumn{7}{|l|}{ Refined Petroleum Product Prices ${ }^{6}$} \\
\hline Distillate Fuel $\ldots \ldots \ldots \ldots \ldots \ldots$ & 98.6 & 96.3 & 99.4 & 109.7 & 108.1 & 105.0 \\
\hline Jet Fuel $\ldots \ldots \ldots \ldots \ldots \ldots \ldots \ldots \ldots \ldots \ldots \ldots \ldots \ldots \ldots \ldots$ & 59.2 & 53.3 & 57.9 & 73.7 & 72.2 & 69.3 \\
\hline Liquefied Petroleum Gas $\ldots \ldots \ldots \ldots \ldots \ldots \ldots \ldots$ & 52.3 & 59.9 & 59.0 & 66.7 & 65.7 & 65.8 \\
\hline Motor Gasoline $\ldots \ldots \ldots \ldots \ldots \ldots \ldots \ldots \ldots \ldots$ & 113.9 & 111.6 & 119.8 & 132.1 & 130.3 & 126.5 \\
\hline Residual Fuel $\ldots \ldots \ldots \ldots \ldots \ldots \ldots \ldots \ldots \ldots$ & 36.3 & 35.8 & 37.6 & 43.8 & 42.8 & 40.5 \\
\hline Residual Fuel (dollars per barrel) . . . . . . . . . . . . . . . & 15.24 & 15.04 & 15.81 & 18.39 & 17.96 & 17.02 \\
\hline Average $\ldots \ldots \ldots \ldots \ldots \ldots \ldots \ldots \ldots \ldots \ldots \ldots$ & 91.2 & 89.7 & 94.7 & 105.9 & 104.3 & 101.5 \\
\hline
\end{tabular}


Table B11. Petroleum Product Prices (Continued) (1994 Cents per Gallon Unless Otherwise Noted)

\begin{tabular}{|c|c|c|c|c|c|c|c|c|c|c|c|}
\hline \multicolumn{9}{|c|}{ Projections } & \multirow{2}{*}{\multicolumn{3}{|c|}{$\begin{array}{c}\text { Annual Growth } \\
\text { 1994-2015 (percent) }\end{array}$}} \\
\hline \multicolumn{3}{|c|}{2005} & \multicolumn{3}{|c|}{2010} & \multicolumn{3}{|c|}{2015} & & & \\
\hline $\begin{array}{c}\text { Slow } \\
\text { Technology }\end{array}$ & Reference & $\begin{array}{c}\text { Rapid } \\
\text { Technology }\end{array}$ & $\begin{array}{c}\text { Slow } \\
\text { Technology }\end{array}$ & Reference & $\begin{array}{c}\text { Rapid } \\
\text { Technology }\end{array}$ & $\begin{array}{c}\text { Slow } \\
\text { Technology }\end{array}$ & Reference & $\begin{array}{c}\text { Rapid } \\
\text { Technology }\end{array}$ & $\begin{array}{c}\text { Slow } \\
\text { Technology }\end{array}$ & Reference & $\begin{array}{c}\text { Rapid } \\
\text { Technology }\end{array}$ \\
\hline 22.99 & 21.86 & 19.77 & 25.59 & 23.70 & 20.55 & 28.58 & 25.43 & 21.17 & 3.0 & 2.4 & 1.5 \\
\hline 111.9 & 109.4 & 103.3 & 118.7 & 113.6 & 105.0 & 124.0 & 115.8 & 104.2 & 1.7 & 1.3 & 0.8 \\
\hline 108.7 & 107.5 & 103.5 & 116.7 & 112.4 & 104.7 & 122.8 & 114.5 & 93.9 & 1.5 & 1.1 & 0.2 \\
\hline 81.5 & 78.9 & 72.4 & 88.1 & 83.1 & 74.0 & 93.5 & 85.0 & 73.0 & 2.0 & 1.5 & 0.8 \\
\hline 54.9 & 52.0 & 46.5 & 61.4 & 56.8 & 48.8 & 68.3 & 60.7 & 51.0 & 2.3 & 1.7 & 0.9 \\
\hline 23.05 & 21.86 & 19.54 & 25.80 & 23.88 & 20.50 & 28.70 & 25.47 & 21.40 & 2.3 & 1.7 & 0.9 \\
\hline 81.9 & 79.3 & 73.0 & 88.7 & 83.7 & 74.8 & 94.2 & 85.8 & 74.0 & 1.9 & 1.4 & 0.7 \\
\hline 62.2 & 61.6 & 57.8 & 68.5 & 64.7 & 56.5 & 73.7 & 65.5 & 45.2 & 1.6 & 1.0 & -0.7 \\
\hline 52.9 & 50.2 & 44.9 & 59.2 & 54.8 & 47.0 & 66.2 & 58.7 & 48.8 & 2.7 & 2.2 & 1.3 \\
\hline 22.22 & 21.07 & 18.86 & 24.86 & 23.01 & 19.74 & 27.81 & 24.64 & 20.50 & 2.7 & 2.2 & 1.3 \\
\hline 127.7 & 124.8 & 117.8 & 131.9 & 126.5 & 116.7 & 136.1 & 127.7 & 114.1 & 1.0 & 0.6 & 0.1 \\
\hline 82.4 & 79.3 & 72.2 & 89.7 & 83.2 & 74.3 & 96.2 & 85.9 & 72.9 & 2.8 & 2.3 & 1.5 \\
\hline 140.0 & 135.2 & 125.4 & 146.1 & 137.3 & 123.6 & 147.4 & 135.1 & 117.2 & 1.3 & 0.9 & 0.2 \\
\hline 49.5 & 46.8 & 41.6 & 55.9 & 51.4 & 43.8 & 63.3 & 55.6 & 45.8 & 3.6 & 3.0 & 2.0 \\
\hline 20.78 & 19.64 & 17.49 & 23.49 & 21.60 & 18.39 & 26.60 & 23.36 & 19.24 & 3.6 & 3.0 & 2.0 \\
\hline 76.4 & 73.8 & 68.1 & 81.9 & 77.2 & 69.3 & 87.1 & 79.0 & 67.1 & 2.2 & 1.8 & 0.9 \\
\hline 54.3 & 51.3 & 45.9 & 61.1 & 56.1 & 48.0 & 68.2 & 60.3 & 50.6 & 2.7 & 2.1 & 1.2 \\
\hline 22.79 & 21.56 & 19.27 & 25.65 & 23.58 & 20.18 & 28.63 & 25.34 & 21.24 & 2.7 & 2.1 & 1.2 \\
\hline 115.0 & 112.2 & 105.3 & 120.2 & 114.9 & 105.6 & 124.2 & 116.6 & 103.7 & 1.2 & 0.9 & 0.4 \\
\hline 82.4 & 79.3 & 72.2 & 89.7 & 83.2 & 74.3 & 96.2 & 85.9 & 72.9 & 2.8 & 2.3 & 1.5 \\
\hline 70.3 & 69.4 & 66.2 & 77.4 & 73.2 & 66.3 & 83.4 & 74.6 & 56.5 & 1.6 & 1.1 & -0.3 \\
\hline 139.8 & 135.0 & 125.2 & 145.9 & 137.1 & 123.4 & 147.3 & 134.9 & 117.1 & 1.3 & 0.9 & 0.2 \\
\hline 52.0 & 49.1 & 43.6 & 58.6 & 53.9 & 45.7 & 65.8 & 57.8 & 47.9 & 2.9 & 2.3 & 1.4 \\
\hline 21.84 & 20.63 & 18.30 & 24.61 & 22.62 & 19.20 & 27.65 & 24.27 & 20.11 & 2.9 & 2.3 & 1.4 \\
\hline 112.2 & 108.5 & 101.2 & 118.0 & 110.6 & 100.5 & 120.8 & 110.2 & 95.5 & 1.4 & 1.0 & 0.3 \\
\hline
\end{tabular}

Includes cogenerators.

${ }^{2}$ Includes Federal and State taxes on diesel fuel and excludes county and local taxes.

3Kerosene-type jet fuel.

¿Sales weighted average price for all grades. Includes Federal and State taxes and excludes county and local taxes.

'Includes all electric power generators except cogenerators, which produce electricity as a by-product of other processes.

Weighted averages of end-use fuel prices are derived from the prices in each sector and the corresponding sectoral consumption.

Sources: 1993 prices for gasoline, distillate, and jet fuel are based on prices in the Energy Information Administration (EIA), Petroleum Marketing Annual 1993, DOE/EIA0487(93) (Washington, DC, July 1994). 1994 prices for gasoline, distillate, and jet fuel are based on prices in various 1994 issues of ElA, Petroleum Marketing Monthly,

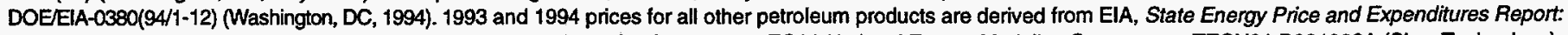

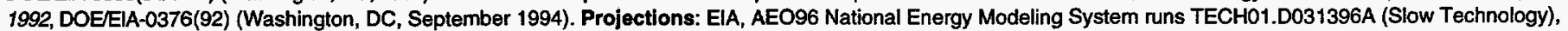
AEO96B.D101995C (reference), and TECH04.D031396B (Rapid Technology). 
Table B12. Natural Gas Supply, Disposition, and Prices

(Trillion Cubic Feet per Year, Unless Otherwise Noted)

\begin{tabular}{|c|c|c|c|c|c|c|}
\hline \multirow{3}{*}{ Supply, Disposition, and Prices } & \multirow[b]{3}{*}{1993} & \multirow[b]{3}{*}{1994} & \multirow[b]{3}{*}{1995} & \multicolumn{3}{|c|}{ Projections } \\
\hline & & & & \multicolumn{3}{|c|}{2000} \\
\hline & & & & $\begin{array}{c}\text { Slow } \\
\text { Technology }\end{array}$ & Reference & $\begin{array}{c}\text { Rapid } \\
\text { Technology } \\
\end{array}$ \\
\hline \multicolumn{7}{|l|}{ Production } \\
\hline Dry Gas Production ${ }^{1}$ & 18.42 & 18.84 & 18.90 & 20.08 & 19.65 & 18.28 \\
\hline Supplemental Natural Gas ${ }^{2} \ldots \ldots \ldots \ldots \ldots \ldots \ldots$ & 0.12 & 0.12 & 0.13 & 0.09 & 0.09 & 0.09 \\
\hline Net Imports $\ldots \ldots \ldots \ldots \ldots \ldots \ldots \ldots \ldots \ldots \ldots \ldots \ldots \ldots$ & 2.21 & 2.40 & 2.56 & 2.98 & 2.98 & 2.98 \\
\hline 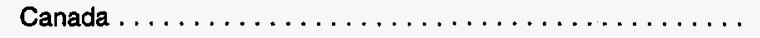 & 2.22 & 2.45 & 2.65 & 2.87 & 2.87 & 2.87 \\
\hline Mexico $\ldots \ldots \ldots \ldots \ldots \ldots \ldots \ldots \ldots \ldots \ldots \ldots \ldots$ & -0.04 & -0.04 & -0.03 & -0.05 & -0.05 & -0.05 \\
\hline Liquefied Natural Gas . . . . . . . . . . . . . . . . . . & 0.03 & -0.01 & -0.05 & 0.16 & 0.16 & 0.16 \\
\hline Total Supply $\ldots \ldots \ldots \ldots \ldots \ldots \ldots \ldots \ldots \ldots$ & 20.74 & 21.36 & 21.60 & 23.14 & 22.71 & 21.35 \\
\hline \multicolumn{7}{|l|}{ Consumption by Sector } \\
\hline 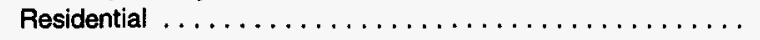 & 4.94 & 4.87 & 4.84 & 5.12 & 5.07 & 4.95 \\
\hline 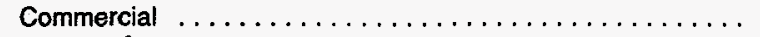 & 2.89 & 2.92 & 2.95 & 3.11 & 3.10 & 2.99 \\
\hline Industria| $^{3} \ldots \ldots \ldots \ldots \ldots \ldots \ldots \ldots \ldots \ldots \ldots$ & 7.62 & 7.83 & 8.23 & 9.12 & 8.90 & 8.72 \\
\hline Electric Generators $^{4} \ldots \ldots \ldots \ldots \ldots \ldots \ldots$ & 2.94 & 3.25 & 3.39 & 3.46 & 3.33 & 2.45 \\
\hline Lease and Plant Fuel ${ }^{5} \ldots \ldots \ldots \ldots \ldots \ldots \ldots$ & 1.18 & 1.24 & 1.24 & 1.30 & 1.28 & 1.24 \\
\hline Pipeline Fuel $\ldots \ldots \ldots \ldots \ldots \ldots \ldots \ldots \ldots \ldots \ldots$ & 0.62 & 0.64 & 0.66 & 0.66 & 0.65 & 0.62 \\
\hline 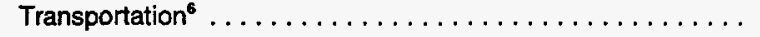 & 0.01 & 0.01 & 0.01 & 0.01 & 0.01 & 0.01 \\
\hline Total $\ldots \ldots \ldots \ldots \ldots \ldots \ldots \ldots \ldots \ldots$ & 20.20 & 20.75 & 21.32 & 22.77 & 22.34 & 20.97 \\
\hline Discrepancy $^{7} \ldots \ldots \ldots \ldots \ldots \ldots \ldots \ldots \ldots \ldots$ & 0.54 & 0.61 & 0.28 & 0.37 & 0.37 & 0.37 \\
\hline \multicolumn{7}{|l|}{ Source Price (1994 dollars per thousand cubic feet) } \\
\hline Average Lower 48 Wellhead Price ${ }^{8} \ldots \ldots \ldots \ldots \ldots$ & 2.09 & 1.88 & 1.60 & 1.93 & 1.89 & 1.75 \\
\hline Average Import Price $\ldots \ldots \ldots$. . & 2.06 & 1.85 & 1.55 & 1.81 & 1.79 & 1.56 \\
\hline Average $^{0} \ldots \ldots \ldots \ldots \ldots \ldots \ldots \ldots \ldots \ldots \ldots$ & 2.08 & 1.87 & 1.59 & 1.92 & 1.88 & 1.72 \\
\hline \multicolumn{7}{|l|}{ Delivered Price (1994 dollars per thousand cubic feet) } \\
\hline 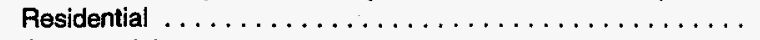 & 6.26 & 6.40 & 6.13 & 6.29 & 6.26 & 6.12 \\
\hline Commercial $\ldots \ldots \ldots \ldots \ldots \ldots \ldots \ldots \ldots \ldots \ldots$ & 5.26 & 5.44 & 5.06 & 5.32 & 5.30 & 5.15 \\
\hline 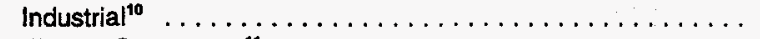 & 2.91 & 2.64 & 2.37 & 2.70 & 2.65 & 2.49 \\
\hline Electric Generators ${ }^{11} \ldots \ldots$. & 2.61 & 2.24 & 2.09 & 2.29 & 2.24 & 2.05 \\
\hline Transportation $^{12} \ldots \ldots \ldots \ldots \ldots \ldots \ldots \ldots \ldots$ & 5.69 & 6.19 & 6.26 & 6.79 & 6.75 & 6.60 \\
\hline Average $^{13} \ldots \ldots \ldots \ldots \ldots \ldots \ldots \ldots \ldots \ldots \ldots$ & 4.14 & 3.98 & 3.67 & 3.91 & 3.89 & 3.80 \\
\hline
\end{tabular}

'Market production (wet) minus extraction losses.

${ }^{2}$ Synthetic natural gas, propane air, cake oven gas, refinery gas, biomass gas, air injected for Btu stabilization, and manufactured gas commingled and distributed with natural gas.

Includes consumption by cogenerators.

4includes all electric power generators except cogenerators, which produce electricity as a by-product of other processes.

BRepresents natural gas used in the field gathering and processing plant machinery.

"Compressed natural gas used as vehicle fuel.

${ }^{7}$ Balancing item. 1993 and 1994 values reflect net storage injections plus natural gas lost as a result of converting flow data measured at varying temperatures and

pressures to a standard temperature and pressure and the merger of different data reporting systems which vary in scope, format, definition, and respondent type.

'Represents lower 48 onshore and offshore supplies.

'Quantity-weighted average of the average lower 48 wellhead price and the average price of imports at the United States border.

${ }^{10}$ Includes consumption by cogenerators.

"Includes all electric power generators except cogenerators, which produce electricity as a by-product of other processes.

${ }^{12}$ Compressed natural gas used as a vehicle fuel.

${ }^{12}$ Weighted average prices and margins. Weights used are the sectoral consumption values excluding lease, plant, and pipeline fuel. 
Table B12. Natural Gas Supply, Disposition, and Prices (Continued) (Trillion Cubic Feet per Year)

\begin{tabular}{|c|c|c|c|c|c|c|c|c|c|c|c|}
\hline \multicolumn{9}{|c|}{ Projections } & \multirow{2}{*}{\multicolumn{3}{|c|}{$\begin{array}{c}\text { Annual Growth } \\
1994-2015 \text { (percent) }\end{array}$}} \\
\hline \multicolumn{3}{|c|}{2005} & \multicolumn{3}{|c|}{2010} & \multicolumn{3}{|c|}{2015} & & & \\
\hline $\begin{array}{c}\text { Slow } \\
\text { Technology } \\
\end{array}$ & Reference & $\begin{array}{c}\text { Rapid } \\
\text { Technology }\end{array}$ & $\begin{array}{c}\text { Slow } \\
\text { Technology }\end{array}$ & Reference & $\begin{array}{c}\text { Rapid } \\
\text { Technology }\end{array}$ & $\begin{array}{c}\text { Slow } \\
\text { Technology }\end{array}$ & Reference & \begin{tabular}{|c|} 
Rapid \\
Technology \\
\end{tabular} & $\begin{array}{c}\text { Slow } \\
\text { Technology }\end{array}$ & Reference & $\begin{array}{c}\text { Rapid } \\
\text { Technology }\end{array}$ \\
\hline 21.89 & 21.25 & 18.76 & 23.70 & 22.83 & 19.88 & 24.83 & 24.97 & 22.36 & 1.3 & 1.3 & 0.8 \\
\hline 0.06 & 0.06 & 0.06 & 0.06 & 0.06 & 0.06 & 0.08 & 0.06 & 0.06 & -1.9 & -3.1 & -3.1 \\
\hline 3.12 & 3.12 & 3.12 & 3.46 & 3.46 & 2.99 & 4.02 & 4.02 & 2.58 & 2.5 & 2.5 & 0.3 \\
\hline 2.90 & 2.90 & 2.90 & 3.11 & 3.11 & 2.64 & 3.42 & 3.42 & 1.98 & 1.6 & 1.6 & -1.0 \\
\hline-0.03 & -0.03 & -0.03 & 0.10 & 0.10 & 0.10 & 0.35 & 0.35 & 0.35 & N/A & N/A & N/A \\
\hline 0.25 & 0.25 & 0.25 & 0.25 & 0.25 & 0.25 & 0.25 & 0.25 & 0.25 & N/A & $N / A$ & $N / A$ \\
\hline 25.07 & 24.43 & 21.94 & 27.22 & 26.35 & 22.93 & 28.92 & 29.04 & 25.00 & 1.5 & 1.5 & 0.8 \\
\hline 5.30 & 5.17 & 4.97 & 5.51 & 5.33 & 5.01 & 5.70 & 5.44 & 5.04 & 0.8 & 0.5 & 0.2 \\
\hline 3.22 & 3.21 & 3.06 & 3.29 & 3.32 & 3.15 & 3.33 & 3.41 & 3.25 & 0.6 & 0.7 & 0.5 \\
\hline 9.71 & 9.40 & 8.96 & 9.92 & 9.72 & 9.04 & 9.80 & 9.91 & 8.73 & 1.1 & 1.1 & 0.5 \\
\hline 4.37 & 4.20 & 2.67 & 5.74 & 5.29 & 3.28 & 7.09 & 7.30 & 5.23 & 3.8 & 3.9 & 2.3 \\
\hline 1.41 & 1.38 & 1.28 & 1.52 & 1.48 & 1.35 & 1.60 & 1.59 & 1.48 & 1.2 & 1.2 & 0.9 \\
\hline 0.69 & 0.69 & 0.63 & 0.76 & 0.74 & 0.66 & 0.83 & 0.83 & 0.75 & 1.2 & 1.3 & 0.8 \\
\hline 0.03 & 0.03 & 0.03 & 0.13 & 0.11 & 0.10 & 0.24 & 0.21 & 0.17 & 18.9 & 18.1 & 17.1 \\
\hline 24.72 & 24.08 & 21.59 & 26.87 & 26.00 & 22.59 & 28.60 & 28.70 & 24.65 & 1.5 & 1.6 & 0.8 \\
\hline 0.35 & 0.35 & 0.35 & 0.35 & 0.35 & 0.35 & 0.32 & 0.35 & 0.35 & NA & $\mathbf{N} / \mathbf{A}$ & N/A \\
\hline 2.23 & 1.99 & 1.52 & 2.99 & 2.15 & 1.38 & 4.18 & 2.57 & 1.55 & 3.9 & 1.5 & -0.9 \\
\hline 2,20 & 1.90 & 1.46 & 2.94 & 2.08 & 1.33 & 4.15 & 2.53 & 1.48 & 3.9 & 1.5 & -1.0 \\
\hline 2.22 & 1.98 & 1.51 & 2.98 & 2.14 & 1.37 & 4.18 & 2.57 & 1.54 & 3.9 & 1.5 & -0.9 \\
\hline 6.39 & 6.15 & 5.72 & 6.99 & 6.08 & 5.29 & 8.34 & 6.59 & 5.34 & 1.3 & 0.1 & -0.9 \\
\hline 5.44 & 5.21 & 4.78 & 6.06 & 5.19 & 4.42 & 7.37 & 5.70 & 4.50 & 1.5 & 0.2 & -0.9 \\
\hline 2.97 & 2.72 & 2.26 & 3.70 & 2.84 & 2.08 & 4.94 & 3.30 & 2.20 & 3.0 & 1.1 & -0.9 \\
\hline 2.56 & 2.31 & 1.80 & 3.40 & 2.49 & 1.64 & 4.68 & 3.02 & 1.83 & 3.6 & 1.4 & -1.0 \\
\hline 6.97 & 6.71 & 6.25 & 7.57 & 6.67 & 5.85 & 9.05 & 7.37 & 6.21 & 1.8 & 0.8 & 0.0 \\
\hline 4.05 & 3.82 & 3.47 & 4.71 & 3.84 & 3.17 & 5.97 & 4.25 & 3.19 & 1.9 & 0.3 & -1.0 \\
\hline
\end{tabular}

Btu $=$ British thermal unit

N/A = Not applicable.

Note: Totals may not equal sum of components due to independent rounding. Figures for 1993 and 1994 may differ from published data due to internal conversion factors.

Sources: 1993 supply values and consumption as lease, plant, and pipeline fuel: Energy Information Administration (ElA), Natura/ Gas Annual 1993, DOE/EIA-0131(93) (Washington, DC, October 1994) with adjustments to end-use sector consumption levels based on Form ElA-867, "Annual Nonutility Power Producer Report." Other 1993 consumption: EIA, State Energy Data Report 1993, DOE/EIA-0214(93) (Washington, DC, July 1995) with adjustments to end-use sector consumption levels based on Form EIA-867, “Annual Nonutility Power Producer Report” 1994 supply values and consumption as lease, plant, and pipeline fuel: EIA, Natural Gas Monthly, DOE/EIA-0130(95/6) (Washington, DC, June 1995). 1994 transportation sector consumption: EIA, AEO96 National Energy Modeling System runs TECH01.D031396A (Slow Technology), AEO96B.D101995C (reference), and TECHO4.D031396B (Rapid Technology). Other 1994 consumption: EIA, Short-Term Energy Outlook, DOE/EIA-0202(95/3Q) (Washington, DC, August 1995) with adjustments to end-use sector consumption levels for consumption of natural gas by electric wholesale generators based on EIA, AEO96 National Energy Modeling System runs TECH01.D031396A (Slow Technology), AEO96B.D101995C (reference), and TECH04.D031396B (Rapid Technology). 1993 residential, commercial, and transportation delivered prices; average lower 48 wellhead price; and average import price: EIA, Natural Gas Annual 1993, DOE/EIA-0131(93) (Washington, DC, October 1994). 1993 electric generators delivered price: Form FERC-423, "Monthly Report of Cost and Quality of Fuels for Electric Plants". 1993 and 1994 industrial delivered prices based on EIA, Manufacturing Energy Consumption Survey, 1991. 1994 residential and commercial delivered prices, average lower 48 wellhead price, and average import price: EIA, Natural Gas Monthy, DOEJEIA-0130(95/6) (Washington, DC, June 1995). Projections: EIA, AEO96 National Energy Modeling System runs TECH01.D031396A (Slow Technology), AEO96B.D101995C (reference), and TECH04.D031396B (Rapid Technology). 


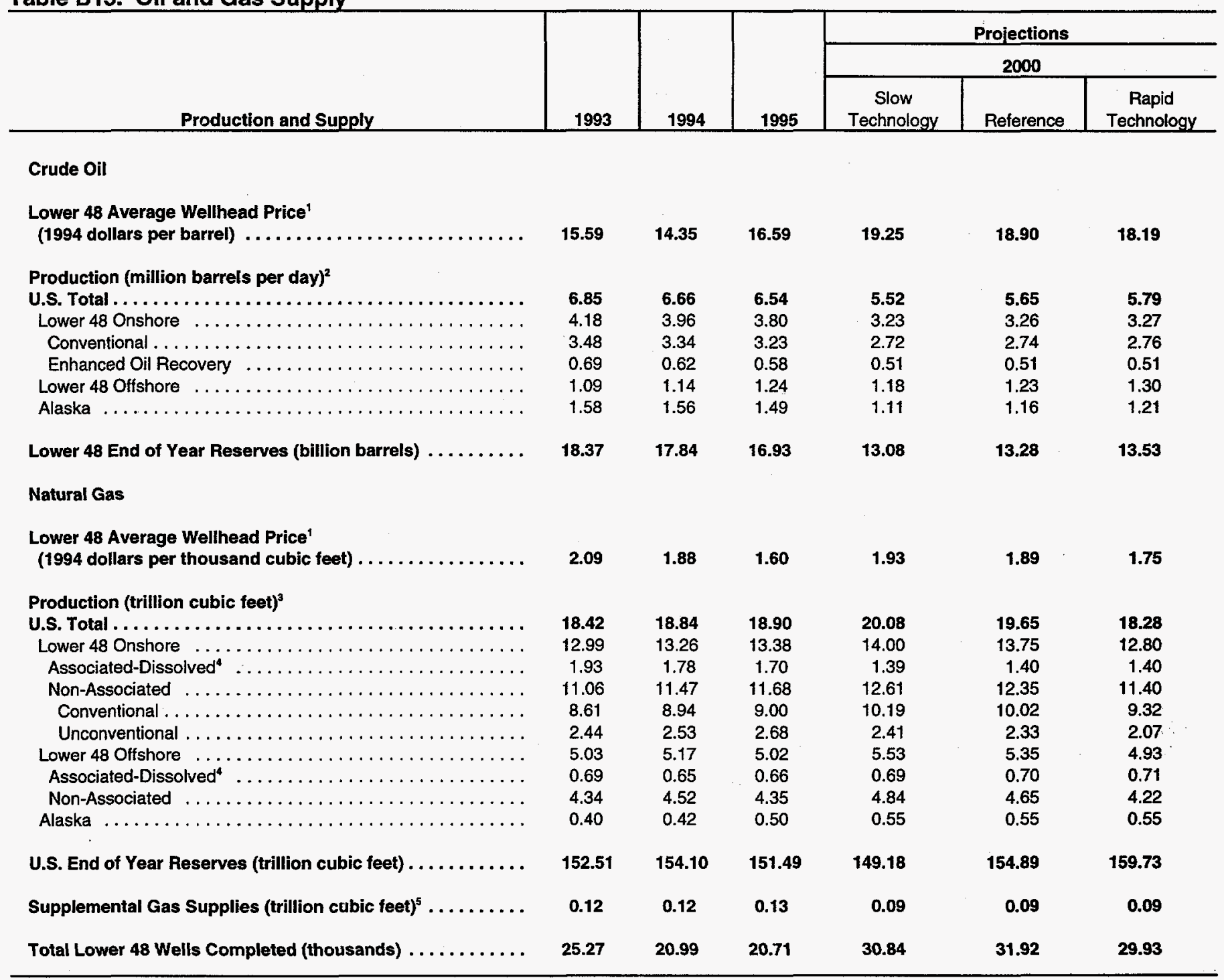


Table B13. Oil and Gas Supply (Continued)

\begin{tabular}{c|c|c|c|c|c|c|c|c|c|c|}
\hline \multicolumn{9}{c|}{ Projections } & \multicolumn{2}{c|}{$\begin{array}{c}\text { Annual Growth } \\
1994-2015 \text { (percent) }\end{array}$} \\
\hline $\begin{array}{c}\text { Slow } \\
\text { Technology }\end{array}$ & Reference & $\begin{array}{c}\text { Rapid } \\
\text { Technology }\end{array}$ & $\begin{array}{c}\text { Slow } \\
\text { Technology }\end{array}$ & $\begin{array}{c}2010 \\
\text { Reference }\end{array}$ & $\begin{array}{c}\text { Rapid } \\
\text { Technology }\end{array}$ & $\begin{array}{c}\text { Slow } \\
\text { Technology }\end{array}$ & Reference & $\begin{array}{c}\text { Rapid } \\
\text { Technology }\end{array}$ & $\begin{array}{c}\text { Slow } \\
\text { Technology }\end{array}$ & $\begin{array}{c}\text { Rapid } \\
\text { Reference }\end{array}$ \\
\hline
\end{tabular}

\begin{tabular}{|c|c|c|c|c|c|c|c|c|c|c|c|}
\hline 22.24 & 21.22 & 19.35 & 24.58 & 22.87 & 20.02 & 27.32 & 24.46 & 20.60 & 3.1 & 2.6 & 1.7 \\
\hline 5.02 & 5.25 & 5.49 & 5.14 & 5.44 & 5.59 & 5.39 & 5.81 & 6.04 & -1.0 & -0.6 & -0.5 \\
\hline 3.33 & 3.38 & 3.29 & 3.67 & 3.75 & 3.58 & 3.96 & 4.10 & 3.94 & 0.0 & 0.2 & 0.0 \\
\hline 2.70 & 2.75 & 2.71 & 2.85 & 2.94 & 2.85 & 2.98 & 3.13 & 3.09 & -0.5 & -0.3 & -0.4 \\
\hline 0.63 & 0.62 & 0.59 & 0.82 & 0.82 & 0.73 & 0.98 & 0.97 & 0.85 & 2.2 & 2.1 & 1.5 \\
\hline 0.97 & 1.06 & 1.27 & 0.84 & 0.98 & 1.23 & 0.77 & 0.98 & 1.29 & -1.8 & -0.7 & 0.6 \\
\hline 0.73 & 0.80 & 0.92 & 0.63 & 0.71 & 0.78 & 0.65 & 0.73 & 0.80 & -4.1 & -3.5 & -3.1 \\
\hline 12.32 & 12.65 & 12.66 & 12.96 & 13.44 & 13.24 & 13.77 & 14.52 & $14: 48$ & -1.2 & -1.0 & -1.0 \\
\hline 2.23 & 1.99 & 1.52 & 2.99 & 2.15 & 1.38 & 4.18 & 2.57 & 1.55 & 3.9 & 1.5 & -0.9 \\
\hline 21.89 & 21.25 & 18.76 & 23.70 & 22.83 & 19.88 & 24.83 & 24.97 & 22.36 & 1.3 & 1.3 & 0.8 \\
\hline 15.92 & 15.41 & 13.39 & 17.48 & 16.45 & 13.51 & 18.29 & 17.19 & 13.77 & 1.5 & 1.2 & 0.2 \\
\hline 1.32 & 1.32 & 1.29 & 1.41 & 1.40 & 1.29 & 1.49 & 1.47 & 1.35 & -0.9 & -0.9 & -1.3 \\
\hline 14.60 & 14.08 & 12.10 & 16.07 & 15.05 & 12.22 & 16.80 & 15.71 & 12.42 & 1.8 & 1.5 & 0.4 \\
\hline 11.91 & 11.54 & 9.98 & 13.04 & 12.13 & 9.96 & 13.34 & 12.29 & 9.85 & 1.9 & 1.5 & 0.5 \\
\hline 2.69 & 2.55 & 2.12 & 3.03 & 2.92 & 2.26 & 3.46 & 3.43 & 2.57 & 1.5 & 1.5 & 0.1 \\
\hline 5.37 & 5.25 & 4.78 & 5.57 & 5.74 & 5.74 & 5.84 & 7.09 & 7.92 & 0.6 & 1.5 & 2.0 \\
\hline 0.64 & 0.66 & 0.70 & 0.60 & 0.64 & 0.70 & 0.58 & 0.64 & 0.71 & -0.5 & -0.1 & 0.4 \\
\hline 4.74 & 4.59 & 4.07 & 4.97 & 5.10 & 5.04 & 5.25 & 6.45 & 7.21 & 0.7 & 1.7 & 2.2 \\
\hline 0.60 & 0.60 & 0.59 & 0.65 & 0.65 & 0.64 & 0.70 & 0.69 & 0.68 & 2.5 & 2.5 & 2.4 \\
\hline 150.19 & 163.38 & 168.54 & 152.46 & 164.42 & 167.07 & 154.71 & 162.00 & 169.22 & 0.0 & 0.2 & 0.4 \\
\hline 0.06 & 0.06 & 0.06 & 0.06 & 0.06 & 0.06 & 0.08 & 0.06 & 0.06 & -1.9 & -3.1 & -3.1 \\
\hline 43.59 & 43.10 & 36.44 & 60.95 & 54.83 & 43.94 & 83.50 & 71.67 & 57.95 & 6.8 & 6.0 & 5.0 \\
\hline
\end{tabular}

'Represents lower 48 onshore and offshore supplies.

${ }^{2}$ Includes lease condensate.

${ }^{3}$ Market production (wet) minus extraction losses.

"Gas which occurs in crude oil reserves either as free gas (associated) or as gas in solution with crude oil (dissolved).

Synthetic natural gas, propane air, coke oven gas, refinery gas, biomass gas, air injected for Btu stabilization, and manufactured gas commingled and distributed with natural gas.

Note: Totals may not equal sum of components due to independent rounding.

Sources: 1993 lower 48 onshore, lower 48 offshore, Alaska crude oil production: Energy Information Administration (EIA), Petroleum Supply Annual 1993, DOE/EIA0340(93)/1 (Washington, DC, June 1993). 1993 U.S. crude oil and natural gas reserves: EIA, U.S. Crude Oil, Natural Gas, and Natural Gas Liquids Reserves, DOE/EIA0216(93) (Washington, DC, October 1994). 1993 natural gas lower 48 average wellhead price, Alaska, total natural gas production, and supplemental gas supplies: ElA, Natural Gas Annual 1993, DOE/EIA-0131(93) (Washington, DC, October 1994). 1993 and 1994 crude oil lower 48 average wellhead price: EIA, Office of Integrated Analysis and Forecasting. 1993 and 1994 total wells completed: EIA, Office of Integrated Analysis and Forecasting. 1994 lower 48 onshore, lower 48 offshore, Alaska crude oil production: EIA, Petroleum Supply Annual 1994, DOE/EIA-0340(94) (Washington, DC, May 1995). 1994 U.S. crude oil reserves and U.S. natural gas reserves: ElA, U.S. Crude Oil, Natural Gas, and Natural Gas Liquids Reserves, Advanced Summary, DOE/ElA-0216(94) (Washington, DC, August 1995). 1994 natural gas lower 48 average wellhead price, total natural gas production: Natural Gas Monthy, DOE/EIA-0130(95/06) (Washington, DC, June 1995). Other 1993 and 1994 values: ElA, Office of Integrated Analysis and Forecasting. Figures for 1993 and 1994 may differ from published data due to internal conversion factors. Projections: ElA, AEO96 National Energy Modeling System runs TECH01.D031396A (Slow Technology), AEO96B.D101995C (reference), and TECHO4.D031396B (Rapid Technology). 
Table B14. Coal Supply, Disposition, and Prices (Million Short Tons per Year, Unless Otherwise Noted)

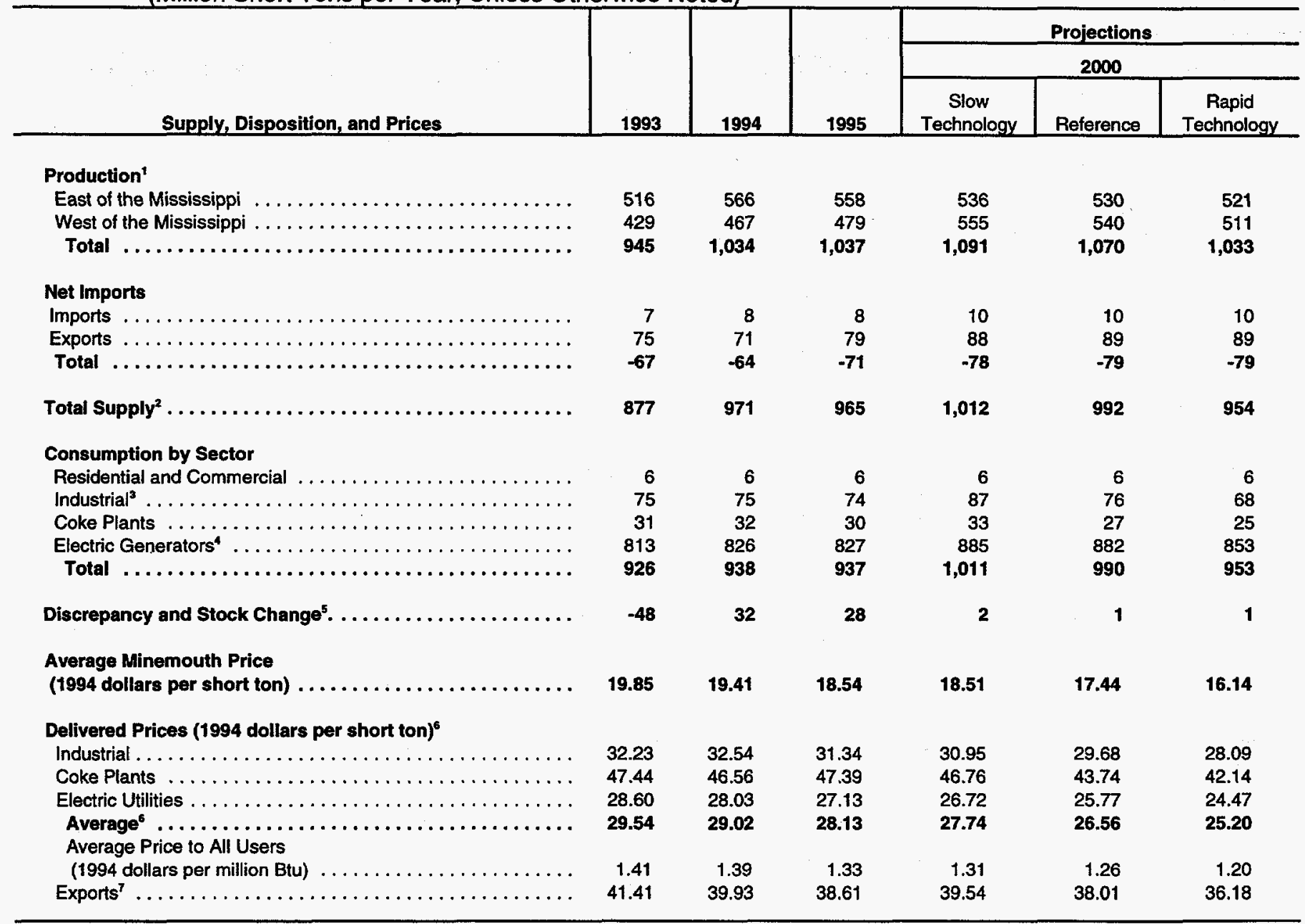


Table B14. Coal Supply, Disposition, and Prices (Continued) (Million Short Tons per Year, Unless Otherwise Noted)

\begin{tabular}{|c|c|c|c|c|c|c|c|c|c|c|c|}
\hline \multicolumn{9}{|c|}{ Projections } & \multirow{2}{*}{\multicolumn{3}{|c|}{$\begin{array}{c}\text { Annual Growth } \\
1994-2015 \text { (percent) }\end{array}$}} \\
\hline \multicolumn{3}{|c|}{2005} & \multicolumn{3}{|c|}{2010} & \multicolumn{3}{|c|}{2015} & & & \\
\hline $\begin{array}{c}\text { Slow } \\
\text { Technology }\end{array}$ & Reference & $\begin{array}{c}\text { Rapid } \\
\text { Technology }\end{array}$ & $\begin{array}{c}\text { Slow } \\
\text { Technology }\end{array}$ & Reference & $\begin{array}{c}\text { Rapid } \\
\text { Technology }\end{array}$ & $\begin{array}{c}\text { Slow } \\
\text { Technology }\end{array}$ & Reference & $\begin{array}{c}\text { Rapid } \\
\text { Technology }\end{array}$ & $\mid \begin{array}{c}\text { Slow } \\
\text { Technology }\end{array}$ & Reference & $\begin{array}{c}\text { Rapid } \\
\text { Technology }\end{array}$ \\
\hline 579 & 557 & 535 & 620 & 584 & 554 & 687 & 617 & 574 & 0.9 & 0.4 & 0.1 \\
\hline 598 & 577 & 535 & 631 & 599 & 552 & 678 & 623 & 573 & 1.8 & 1.4 & 1.0 \\
\hline 1,177 & 1,135 & 1,069 & 1,251 & 1,184 & 1,106 & 1,365 & 1,240 & 1,147 & 1.3 & 0.9 & 0.5 \\
\hline 10 & 10 & 10 & 10 & 10 & 10 & 10 & 10 & 10 & 1.5 & 1.5 & 1.5 \\
\hline 98 & 97 & 97 & 111 & 111 & 111 & 129 & 130 & 130 & 2.9 & 2.9 & 2.9 \\
\hline-87 & -87 & -87 & -100 & -100 & -101 & -119 & -119 & -120 & 3.0 & 3.0 & 3.0 \\
\hline 1,089 & 1,048 & 983 & 1,151 & 1,083 & 1,006 & 1,246 & 1,120 & 1,027 & 1.2 & 0.7 & 0.3 \\
\hline 6 & 6 & 6 & 6 & 6 & 6 & 7 & 6 & 6 & 0.5 & 0.2 & -0.1 \\
\hline 100 & 78 & 61 & 118 & 80 & 57 & 143 & 86 & 55 & 3.1 & 0.6 & -1.4 \\
\hline 31 & 23 & 21 & 30 & 20 & 17 & 30 & 18 & 14 & -0.3 & -2.7 & -3.7 \\
\hline 952 & 939 & 894 & 995 & 975 & 923 & 1065 & 1009 & 950 & 1.2 & 1.0 & 0.7 \\
\hline 1,090 & 1,046 & 982 & 1,150 & 108,2 & 1,003 & 1,244 & 1,120 & 1,025 & 1.4 & 0.8 & 0.4 \\
\hline 0 & 2 & 1 & 1 & 1 & 2 & 1 & 1 & 2 & N/A & N/A & N/A \\
\hline 19.95 & 17.68 & 15.74 & 20.22 & 17.43 & 15.59 & 20.95 & 17.39 & 15.26 & 0.4 & -0.5 & -1.1 \\
\hline 32.88 & 30.06 & 27.43 & 33.95 & 30.13 & 27.15 & 34.14 & 29.81 & 26.86 & 0.2 & -0.4 & -0.9 \\
\hline 49.56 & 44.57 & 42.24 & 49.66 & 44.60 & 41.92 & 50.08 & 44.00 & 41.21 & 0.3 & -0.3 & -0.6 \\
\hline 28.32 & 26.28 & 24.21 & 28.47 & 25.88 & 23.85 & 29.47 & 26.17 & 23.88 & 0.2 & -0.3 & -0.8 \\
\hline 29.35 & 26.97 & 24.80 & 29.60 & 26.55 & 24.35 & 30.50 & 26.74 & 24.29 & 0.2 & -0.4 & -0.8 \\
\hline 1.39 & 1.28 & 1.18 & 1.41 & 1.27 & 1.17 & 1.45 & 1.28 & 1.17 & 0.2 & -0.4 & -0.8 \\
\hline 41.50 & 38.30 & 36.12 & 40.91 & 37.64 & 35.25 & 41.45 & 37.22 & 35.01 & 0.2 & -0.3 & -0.6 \\
\hline
\end{tabular}

1'Includes anthracite, bituminous coal, and lignite.

${ }^{2}$ Production plus net imports plus net storage withdrawals.

includes consumption by cogenerators.

Includes all electric power generators except cogenerators, which produce electricity as a by-product of other processes.

${ }^{5}$ Balancing item: the sum of production, net imports, and net storage minus total consumption.

'Sectoral prices weighted by consumption tonnage; weighted average excludes residential/ commercial prices and export f.a.s. prices.

${ }^{7}$ Free-alongside-ship (t.a.s.) price at United States port-of-exit.

N/A = Not applicable.

Btu $=$ British thermal unit.

Note: Totals may not equal sum of components due to independent rounding.

Sources: 1993 production, imports, exports, consumption, minemouth price, and delivered prices: Energy Information Administration (EIA), Coal Industry Annual, DOEJEIA-0584(93) (Washington, DC, December 1994). 1994 production, imports, exports, consumption, minemouth price, and delivered prices: EIA, Coal Industry Annual, DOE/EIA-0584(94) (Washington, DC, 1995). Projections: EAA, AEO96 National Energy Modeling System runs TECH01.D031396A (Slow Technology), AEO96B.D101995C (reference), and TECH04.D031396B (Rapid Technology). 
Table B15. Renewable Energy Generating Capacity and Generation (Thousand Megawatts, Unless Otherwise Noted)

\begin{tabular}{|c|c|c|c|c|c|c|}
\hline \multirow[b]{3}{*}{ Capacity and Generation } & \multirow[b]{3}{*}{1993} & \multirow[b]{3}{*}{1994} & \multirow[b]{3}{*}{1995} & \multicolumn{3}{|c|}{ Projections } \\
\hline & & & & \multicolumn{3}{|c|}{2000} \\
\hline & & & & $\begin{array}{c}\text { Slow } \\
\text { Technology }\end{array}$ & Reference & $\begin{array}{c}\text { Rapid } \\
\text { Technology }\end{array}$ \\
\hline \multicolumn{7}{|l|}{$\begin{array}{l}\text { Electric Utilities and Nonutilities }{ }^{1} \\
\text { (excluding cogenerators) } \\
\text { Capability }\end{array}$} \\
\hline Conventional Hydropower $\ldots \ldots \ldots \ldots \ldots \ldots \ldots \ldots$ & 78.21 & 78.20 & 78.50 & 80.60 & 80.60 & 80.60 \\
\hline Geothermal $^{2} \ldots \ldots \ldots \ldots \ldots \ldots \ldots \ldots \ldots \ldots$ & 2.97 & 2.97 & 3.01 & 3.21 & 3.21 & 3.21 \\
\hline Municipal Solid Waste $\ldots \ldots \ldots \ldots \ldots \ldots \ldots \ldots \ldots$ & 3.00 & 3.04 & 3.45 & 4.03 & 4.03 & 4.03 \\
\hline Wood and Other Biomass ${ }^{3} \ldots \ldots \ldots \ldots \ldots \ldots \ldots$ & 1.71 & 1.71 & 1.75 & 1.80 & 1.80 & 1.80 \\
\hline Solar Thermal $\ldots \ldots \ldots \ldots \ldots \ldots \ldots \ldots \ldots \ldots$ & 0.35 & 0.35 & 0.36 & 0.40 & 0.40 & 0.40 \\
\hline Solar Photovoltaic . . . . . . . . . . . . . . . . . & 0.01 & 0.01 & 0.01 & 0.02 & 0.02 & 0.02 \\
\hline 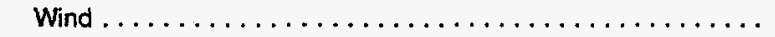 & 1.78 & 1.79 & 2.09 & 2.68 & 2.73 & 3.26 \\
\hline Total $\ldots \ldots \ldots \ldots \ldots \ldots \ldots \ldots \ldots \ldots$ & 88.04 & 88.07 & 89.17 & 92.74 & 92.79 & 93.32 \\
\hline \multicolumn{7}{|l|}{ Generation (billion kilowatthours) } \\
\hline Conventional Hydropower $\ldots \ldots \ldots \ldots \ldots \ldots \ldots \ldots$ & 279.10 & 259.86 & 293.80 & 303.11 & 303.10 & 303.04 \\
\hline Geothermal $^{2} \ldots \ldots \ldots \ldots \ldots \ldots \ldots \ldots \ldots \ldots$ & 17.32 & 17.06 & 17.10 & 18.18 & 18.18 & 18.17 \\
\hline Municipal Solid Waste $\ldots \ldots \ldots \ldots \ldots \ldots \ldots$ & 17.38 & 17.29 & 21.49 & 25.41 & 25.41 & 25.40 \\
\hline Wood and Other Biomass ${ }^{3} \ldots \ldots \ldots \ldots \ldots \ldots$ & 8.64 & 8.90 & 8.00 & 8.32 & 8.32 & 8.32 \\
\hline Solar Thermal $\ldots \ldots \ldots \ldots \ldots \ldots \ldots \ldots \ldots$ & 0.90 & 0.82 & 0.92 & 1.04 & 1.04 & 1.04 \\
\hline 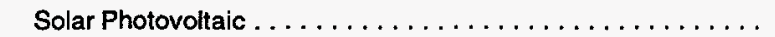 & 0.00 & 0.00 & 0.03 & 0.08 & 0.08 & 0.08 \\
\hline Wind $\ldots \ldots \ldots \ldots \ldots \ldots \ldots \ldots \ldots \ldots \ldots$ & 3.04 & 3.45 & 3.93 & 5.15 & 5.32 & 6.82 \\
\hline Total $\ldots \ldots \ldots \ldots \ldots \ldots \ldots \ldots \ldots \ldots \ldots$ & 326.38 & 307.39 & 345.28 & 361.28 & 361.44 & 362.86 \\
\hline \multicolumn{7}{|l|}{ Cogenerators ${ }^{4}$} \\
\hline Municipal Solid Waste $\ldots \ldots \ldots \ldots \ldots \ldots \ldots \ldots$ & 0.40 & 0.37 & 0.37 & 0.38 & 0.38 & 0.38 \\
\hline Biomass $\ldots \ldots \ldots \ldots \ldots \ldots \ldots \ldots \ldots \ldots$ & 5.54 & 5.72 & 5.81 & 6.10 & 6.10 & 6.10 \\
\hline Total $\ldots \ldots \ldots \ldots \ldots \ldots \ldots \ldots \ldots \ldots$ & 5.94 & 6.09 & 6.18 & 6.48 & 6.48 & 6.48 \\
\hline \multicolumn{7}{|l|}{ Generation (billion kilowatthours) } \\
\hline Municipal Solid Waste $\ldots \ldots \ldots \ldots \ldots \ldots \ldots$ & 1.88 & 1.93 & 1.75 & 1.79 & 1.79 & 1.79 \\
\hline Biomass $\ldots \ldots \ldots \ldots \ldots \ldots \ldots \ldots \ldots \ldots \ldots$ & 36.21 & 37.69 & 38.49 & 40.57 & 40.57 & 40.57 \\
\hline Total $\ldots \ldots \ldots \ldots \ldots \ldots \ldots \ldots \ldots \ldots$ & 38.10 & 39.62 & 40.24 & 42.36 & 42.36 & 42.36 \\
\hline
\end{tabular}


Table B15. Renewable Energy Generating Capacity and Generation (Continued) (Thousand Megawatts, Unless Otherwise Noted)

\begin{tabular}{|c|c|c|c|c|c|c|c|c|c|c|c|}
\hline \multicolumn{9}{|c|}{ Projections } & \multirow{2}{*}{\multicolumn{3}{|c|}{$\begin{array}{c}\text { Annual Growth } \\
\text { 1994-2015(percent) }\end{array}$}} \\
\hline \multicolumn{3}{|c|}{2005} & \multicolumn{3}{|c|}{2010} & \multicolumn{3}{|c|}{2015} & & & \\
\hline $\begin{array}{c}\text { Slow } \\
\text { Technology }\end{array}$ & Reference & $\begin{array}{c}\text { Rapid } \\
\text { Technology }\end{array}$ & $\begin{array}{c}\text { Slow } \\
\text { Technology }\end{array}$ & Reference & $\begin{array}{c}\text { Rapid } \\
\text { Technology }\end{array}$ & $\begin{array}{c}\text { Slow } \\
\text { Technology }\end{array}$ & Reference & $\begin{array}{c}\text { Rapid } \\
\text { Technology }\end{array}$ & $\begin{array}{c}\text { Slow } \\
\text { Technology }\end{array}$ & Reference & $\begin{array}{c}\text { Rapid } \\
\text { Technology }\end{array}$ \\
\hline
\end{tabular}

\begin{tabular}{|c|c|c|c|c|c|c|c|c|c|c|c|}
\hline 80.92 & 80.92 & 80.92 & 80.97 & 80.97 & 80.97 & 80.97 & 80.97 & 80.97 & 0.2 & 0.2 & 0.2 \\
\hline 3.08 & 3.08 & 2.95 & 3.47 & 3.36 & 2.87 & 4.22 & 3.72 & 2.68 & 1.7 & 1.1 & -0.5 \\
\hline 4.43 & 4.43 & 4.43 & 4.84 & 4.85 & 4.86 & 5.22 & 5.23 & 5.25 & 2.6 & 2.6 & 2.6 \\
\hline 1.92 & 1.92 & 1.92 & 1.92 & 1.93 & 1.92 & 2.16 & 2.84 & 2.36 & 1.1 & 2.4 & 1.6 \\
\hline 0.52 & 0.52 & 0.52 & 0.63 & 0.63 & 0.63 & 1.25 & 1.73 & 1.34 & 6.2 & 7.9 & 6.6 \\
\hline 0.07 & 0.07 & 0.07 & 0.18 & 0.18 & 0.18 & 0.32 & 0.32 & 0.78 & 17.6 & 17.6 & 22.7 \\
\hline 3.15 & 3.57 & 3.67 & 3.90 & 5.10 & 4.57 & 9.53 & 11.64 & 7.68 & 8.3 & 9.3 & 7.2 \\
\hline 94.09 & 94.51 & 94.48 & 95.92 & 97.02 & 95.99 & 103.67 & 106.45 & 101.07 & 0.8 & 0.9 & 0.7 \\
\hline 305.63 & 305.60 & 305.48 & 306.21 & 306.16 & 305.97 & 306.43 & 306.35 & 306.08 & 0.8 & 0.8 & 0.8 \\
\hline 17.95 & 17.94 & 16.99 & 21.16 & 20.36 & 16.88 & 27.03 & 23.55 & 16.20 & 2.2 & 1.5 & -0.2 \\
\hline 28.17 & 28.18 & 28.19 & 31.05 & 31.08 & 31.10 & 33.65 & 33.74 & 33.80 & 3.2 & 3.2 & 3.2 \\
\hline 8.86 & 8.86 & 8.86 & 8.86 & 8.89 & 8.86 & 10.53 & 15.26 & 11.94 & 0.8 & 2.6 & 1.4 \\
\hline 1.38 & 1.38 & 1.38 & 1.71 & 1.71 & 1.71 & 3.76 & 5.43 & 4.07 & 7.5 & 9.4 & 7.9 \\
\hline 0.18 & 0.18 & 0.18 & 0.40 & 0.40 & 0.40 & 0.69 & 0.69 & 1.59 & 29.6 & 29.6 & 34.8 \\
\hline 5.76 & 7.19 & 7.20 & 8.14 & 12.33 & 10.43 & 25.46 & 33.37 & 22.00 & 10.0 & 11.4 & 9.2 \\
\hline 367.93 & 369.33 & 368.29 & 377.53 & 380.94 & 375.34 & 407.54 & 418.39 & 395.68 & 1.4 & 1.5 & 1.2 \\
\hline 0.39 & 0.39 & 0.39 & 0.40 & 0.40 & 0.40 & 0.41 & 0.41 & 0.41 & 0.5 & 0.5 & 0.5 \\
\hline 6.76 & 6.76 & 6.75 & 7.24 & 7.22 & 7.19 & 7.75 & 7.70 & 7.62 & 1.5 & 1.4 & 1.4 \\
\hline 7.15 & 7.15 & 7.14 & 7.64 & 7.62 & 7.59 & 8.16 & 8.11 & 8.03 & 1.4 & 1.4 & 1.3 \\
\hline 1.85 & 1.85 & 1.85 & 1.89 & 1.89 & 1.89 & 1.93 & 1.93 & 1.93 & 0.0 & 0.0 & 0.0 \\
\hline 44.99 & 44.96 & 44.92 & 48.20 & 48.09 & 47.89 & 51.61 & 51.28 & 50.78 & 1.5 & 1.5 & 1.4 \\
\hline 46.84 & 46.81 & 46.76 & 50.09 & 49.98 & 49.78 & 53.54 & 53.21 & 52.71 & 1.4 & 1.4 & 1.4 \\
\hline
\end{tabular}

IIncludes traditional utilities and nonutilities other than cogenerators. These nonutility facilities include small power producers, exempt wholesale generators and generators at industrial and commercial facilities who do not produce steam for other uses.

${ }^{2}$ Includes hydrothermal resources only (hot water and steam).

${ }^{3}$ Includes projections for energy crops after 2010.

Includes cogenerators at facilities whose primary function is not electricity production. In general, biomass and other waste facilities are cogenerators while the remaining renewables produce only electricity.

Notes: Totals may not equal sum of components due to independent rounding. Net summer capability has been estimated for nonutility generators for AEO96. Net summer

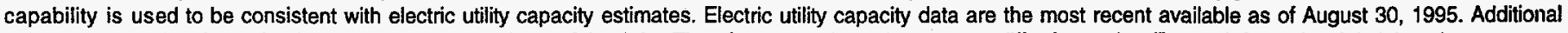
retirements are also determined based on the size and age of the units. Therefore, capacity estimates may differ from other Energy Information Administration sources.

Sources: 1993 and 1994 electric utility capability: Energy Information Administration (EIA), Form EIA-860 "Annual Electric Utility Report." 1993 and 1994 nonutility and cogenerator capability: Form ElA-867, "Annual Nonutility Power Producer Report." 1993 and 1994 generation: ElA, Annual Energy Review 1994, DOE/ElA-0384(94) (Washington, DC, July 1995). Projections: ElA, AEO96 National Energy Modeling System runs TECH01.D031396A (Slow Technology), AEO96B.D101995C (reference), and TECH04.D031396B (Rapid Technology). 
Table B16. Renewable Energy Consumption by Sector and Source (Quadrillion Btu per Year)

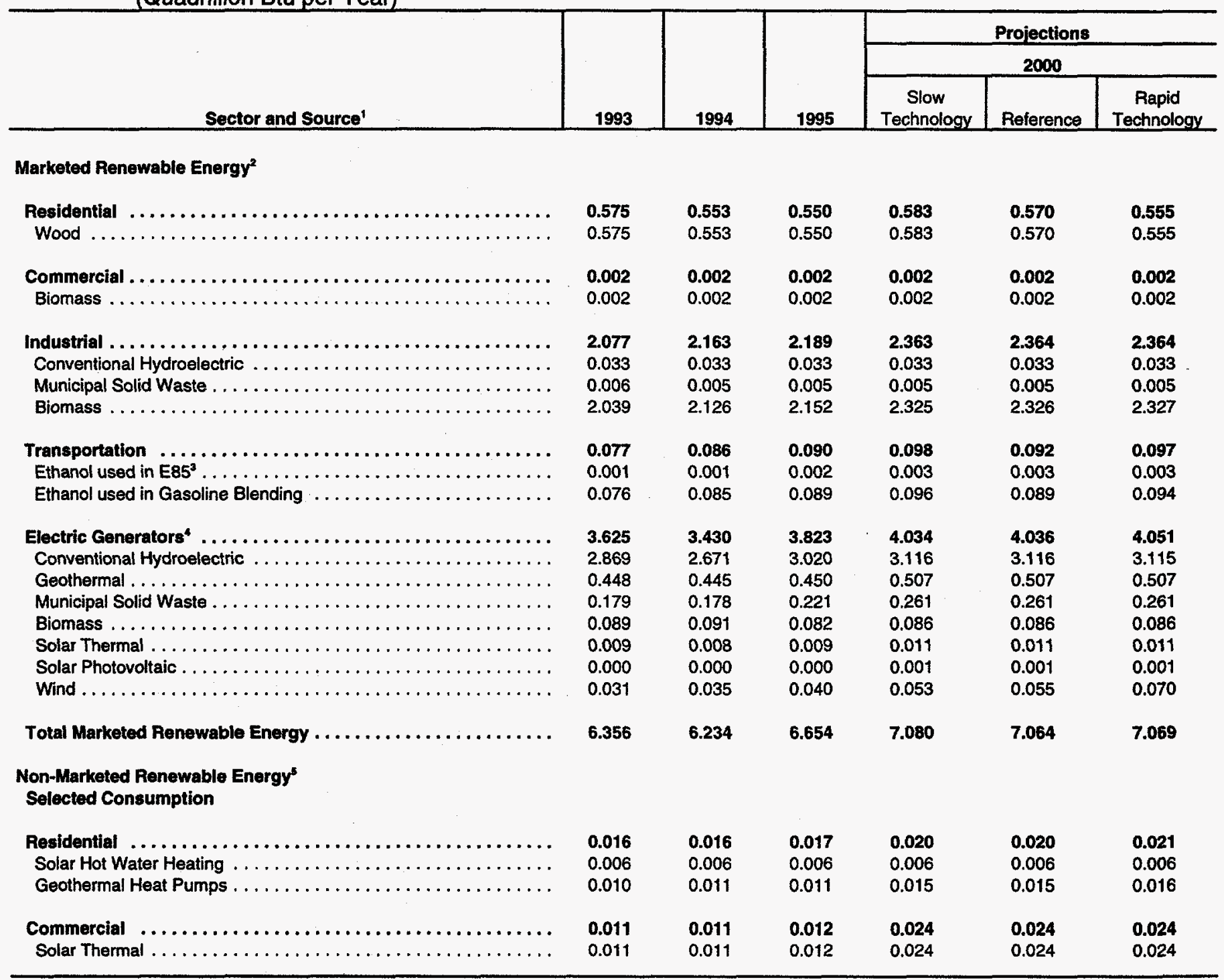


Table B16. Renewable Energy Consumption by Sector and Source (Continued) (Quadrillion Btu per Year)

\begin{tabular}{c|c|c|c|c|c|c|c|c|c|c|}
\hline \multicolumn{9}{c|}{ Projections } & \multicolumn{2}{c|}{$\begin{array}{c}\text { Annual Growth } \\
\text { 1994-2015 (percent) }\end{array}$} \\
\hline $\begin{array}{c}\text { Slow } \\
\text { Technology }\end{array}$ & $\begin{array}{c}2005 \\
\text { Reference }\end{array}$ & $\begin{array}{c}\text { Rapid } \\
\text { Technology }\end{array}$ & $\begin{array}{c}\text { Slow } \\
\text { Technology }\end{array}$ & Reference & $\begin{array}{c}\text { Rapid } \\
\text { Technology }\end{array}$ & $\begin{array}{c}\text { Slow } \\
\text { Technology }\end{array}$ & Reference & $\begin{array}{c}\text { Rapid } \\
\text { Technology }\end{array}$ & $\begin{array}{c}\text { Slow } \\
\text { Technology }\end{array}$ & $\begin{array}{c}\text { Rapid } \\
\text { Reference }\end{array}$ \\
Technology \\
\hline
\end{tabular}

\begin{tabular}{|c|c|c|c|c|c|c|c|c|c|c|c|}
\hline 0.601 & 0.577 & 0.549 & 0.622 & 0.585 & 0.541 & 0.643 & 0.592 & 0.533 & 0.7 & 0.3 & -0.2 \\
\hline 0.601 & 0.577 & 0.549 & 0.622 & 0.585 & 0.541 & 0.643 & 0.592 & 0.533 & 0.7 & 0.3 & -0.2 \\
\hline 0.002 & 0.002 & 0.002 & 0.002 & 0.002 & 0.002 & 0.002 & 0.002 & 0.002 & 0.7 & 0.7 & 0.7 \\
\hline 0.002 & 0.002 & 0.002 & 0.002 & 0.002 & 0.002 & 0.002 & 0.002 & 0.002 & 0.7 & 0.7 & 0.7 \\
\hline 2.562 & 2.564 & 2.565 & 2.699 & 2.707 & 2.705 & 2.848 & 2.862 & 2.857 & 1.3 & 1.3 & 1.3 \\
\hline 0.033 & 0.033 & 0.033 & 0.033 & 0.033 & 0.033 & 0.033 & 0.033 & 0.033 & N/A & N/A & N/A \\
\hline 0.005 & 0.005 & 0.005 & 0.005 & 0.005 & 0.005 & 0.006 & 0.006 & 0.006 & 0.9 & 0.9 & 0.9 \\
\hline 2.524 & 2.527 & 2.528 & 2.662 & 2.669 & 2.667 & 2.810 & 2.824 & 2.819 & 1.3 & 1.4 & 1.4 \\
\hline 0.199 & 0.190 & 0.182 & 0.260 & 0.248 & 0.234 & 0.320 & 0.290 & 0.252 & 6.4 & 5.9 & 5.2 \\
\hline 0.049 & 0.046 & 0.043 & 0.106 & 0.096 & 0.087 & 0.160 & 0.137 & 0.121 & 26.3 & 25.4 & 24.7 \\
\hline 0.150 & 0.145 & 0.139 & 0.154 & 0.152 & 0.147 & 0.159 & 0.152 & 0.131 & 3.0 & 2.8 & 2.1 \\
\hline 4.115 & 4.129 & 4.097 & 4.289 & 4.310 & 4.178 & 4.757 & 4.787 & 4.385 & 1.6 & 1.6 & 1.2 \\
\hline 3.142 & 3.142 & 3.140 & 3.148 & 3.147 & 3.145 & 3.150 & 3.149 & 3.146 & 0.8 & 0.8 & 0.8 \\
\hline 0.517 & 0.516 & 0.486 & 0.626 & 0.604 & 0.493 & 0.845 & 0.728 & 0.484 & 3.1 & 2.4 & 0.4 \\
\hline 0.290 & 0.290 & 0.290 & 0.319 & 0.319 & 0.320 & 0.346 & 0.347 & 0.347 & 3.2 & 3.2 & 3.2 \\
\hline 0.091 & 0.091 & 0.091 & 0.091 & 0.091 & 0.091 & 0.108 & 0.157 & 0.123 & 0.8 & 2.6 & 1.4 \\
\hline 0.014 & 0.014 & 0.014 & 0.018 & 0.018 & 0.018 & 0.039 & 0.056 & 0.042 & 7.5 & 9.4 & 7.9 \\
\hline 0.002 & 0.002 & 0.002 & 0.004 & 0.004 & 0.004 & 0.007 & 0.007 & 0.016 & 29.6 & 29.6 & 34.8 \\
\hline 0.059 & 0.074 & 0.074 & 0.084 & 0.127 & 0.107 & 0.262 & 0.343 & 0.226 & 10.0 & 11.4 & 9.2 \\
\hline .479 & 7.463 & 7.396 & 7.873 & 7.852 & 7.660 & 8.570 & 8.533 & 8.029 & 1.5 & 1.5 & 1.2 \\
\hline
\end{tabular}

\begin{tabular}{|c|c|c|c|c|c|c|c|c|c|c|c|}
\hline 0.024 & 0.025 & 0.026 & 0.027 & 0.029 & 0.030 & 0.030 & 0.034 & 0.034 & 2.9 & 3.5 & 3.5 \\
\hline 0.006 & 0.006 & 0.006 & 0.005 & 0.005 & 0.005 & 0.005 & 0.005 & 0.005 & -0.3 & -0.3 & -0.3 \\
\hline 0.027 & 0.027 & 0.027 & 0.030 & 0.030 & 0.030 & 0.037 & 0.037 & 0.037 & 5.7 & 5.7 & 5.7 \\
\hline
\end{tabular}

${ }^{1}$ Actual heat rates used to determine fuel consumption for all renewable fuels except hydropower, solar, and wind. Consumption at hydroelectric, solar, and wind facilities determined using the fossil fuel equivalent of 10,280 Btu per kilowatthour.

Includes nonelectric renewable energy groups for which the energy source is bought and sold in the marketplace, although individual instances may not necessarily be marketed, and marketed renewable energy inputs for electricity entering the marketplace on the electric power grid.

${ }^{3}$ Excludes motor gasoline component of E85.

Includes renewable energy delivered to the grid from electric utilities, nonutilities, and that part of industrial and other cogeneration delivered to the grid. Renewable energy used in generating electricity for own use is included in the individual sectoral electricity energy consumption values.

Includes selected renewable energy consumption for which the energy is not bought or sold, either directly or indirectly as an input to marketed energy. The Energy Information Administration does not estimate or project total consumption of nonmarketed renewable energy.

N/A $=$ Not available.

Btu = British thermal unit.

Notes: Totals may not equal sum of components due to independent rounding.

Sources: 1993 ethanol: Energy Information Administration (EIA), Annual Energy Review, DOE/EIA-0384(94) (Washington, DC, July 1995). 1993 and 1994 electric generator: EIA, Form EIA-860, "Annual Electric Utility Report" and EIA, Form EIA-867, "Annual Nonutility Power Producer Report." Other 1993: EIA, Office of Integrated Analysis and Forecasting. 1994 ethanol: EIA, Petroleum Supply Annual, 1994, DOE/EIA-0340(94/1) (Washington, DC, June 1995). Other 1994: EIA, Office of Integrated Analysis and Forecasting. Projections: EIA, AEO96 National Energy Modeling System runs TECH01.D031396A (Slow Technology), AEO96B.D101995C (reference), and TECH04.D031396B (Rapid Technology). 


\begin{tabular}{|c|c|c|c|c|c|c|}
\hline \multirow[b]{3}{*}{ Sector and Source } & \multirow[b]{3}{*}{1993} & \multirow[b]{3}{*}{1994} & \multirow[b]{3}{*}{1995} & \multicolumn{3}{|c|}{ Projections } \\
\hline & & & & \multicolumn{3}{|c|}{2000} \\
\hline & & & & $\begin{array}{c}\text { Slow } \\
\text { Technology } \\
\end{array}$ & Reference & $\begin{array}{c}\text { Rapid } \\
\text { Technology }\end{array}$ \\
\hline \multicolumn{7}{|l|}{ Residential } \\
\hline$\ldots \ldots \ldots \ldots \ldots \ldots \ldots \ldots$ & 26.2 & 25.4 & 24.6 & 24.9 & 24.5 & 24.2 \\
\hline 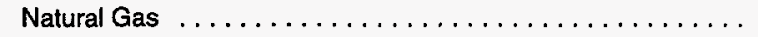 & 73.4 & 72.4 & 71.9 & 76.0 & 75.2 & 73.4 \\
\hline 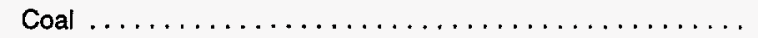 & 1.5 & 1.4 & 1.4 & 1.4 & 1.3 & 1.3 \\
\hline Electricity $\ldots \ldots \ldots \ldots \ldots \ldots \ldots \ldots$ & 170.6 & 170.9 & 170.8 & 181.5 & 179.9 & 170.5 \\
\hline Total $\ldots \ldots \ldots \ldots \ldots \ldots \ldots \ldots \ldots \ldots \ldots \ldots$ & 271.6 & 270.1 & 268.8 & 283.8 & 280.9 & 269.4 \\
\hline \multicolumn{7}{|l|}{ Commercial } \\
\hline 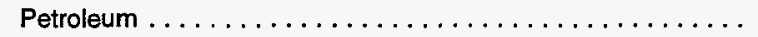 & 14.9 & 14.6 & 14.5 & 14.8 & 14.8 & 15.4 \\
\hline Natural Gas $\ldots \ldots \ldots \ldots \ldots \ldots \ldots \ldots \ldots$ & 43.1 & 43.4 & 43.8 & 46.2 & 46.0 & 44.4 \\
\hline 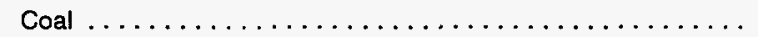 & 2.2 & 2.1 & 2.1 & 2.2 & 2.2 & 2.2 \\
\hline Electricity $\ldots \ldots \ldots \ldots \ldots \ldots \ldots \ldots \ldots$ & 151.8 & 155.7 & 156.5 & 164.4 & 163.3 & 148.4 \\
\hline 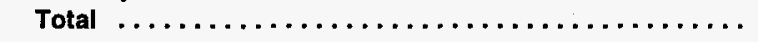 & 212.1 & 215.8 & 217.0 & 227.6 & 226.4 & 210.4 \\
\hline \multicolumn{7}{|l|}{ Industrial ${ }^{t}$} \\
\hline$\ldots \ldots \ldots \ldots \ldots \ldots \ldots \ldots \ldots \ldots$ & 92.5 & 98.7 & 100.6 & 104.6 & 103.4 & 100.9 \\
\hline Natural Gas $^{2} \ldots \ldots \ldots \ldots \ldots \ldots \ldots \ldots$ & 131.6 & 133.5 & 139.2 & 153.6 & 150.1 & 148.1 \\
\hline Coal $\ldots \ldots \ldots \ldots \ldots \ldots \ldots \ldots \ldots \ldots \ldots \ldots$ & 62.3 & 63.9 & 63.0 & 72.3 & 61.7 & 56.7 \\
\hline 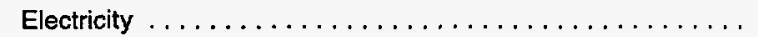 & 167.5 & 168.6 & 167.5 & 176.4 & 175.7 & 168.0 \\
\hline Total $\ldots \ldots \ldots \ldots \ldots \ldots \ldots \ldots \ldots \ldots \ldots \ldots$ & 454.0 & 464.7 & 470.2 & 506.9 & 491.0 & 473.7 \\
\hline \multicolumn{7}{|l|}{ Transportation } \\
\hline Petroleum $\ldots \ldots \ldots \ldots \ldots \ldots \ldots \ldots \ldots \ldots \ldots$ & 426.8 & 436.2 & 446.4 & 483.8 & 480.5 & 468.5 \\
\hline 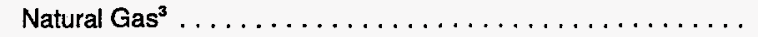 & 9.3 & 9.6 & 9.8 & 9.9 & 9.7 & 9.3 \\
\hline Other $\ldots \ldots \ldots \ldots \ldots \ldots \ldots \ldots \ldots \ldots \ldots$ & 0.7 & 0.0 & 0.0 & 0.0 & 0.0 & 0.0 \\
\hline Electricity $\ldots \ldots \ldots \ldots \ldots \ldots \ldots \ldots \ldots \ldots$ & 0.0 & 1.0 & 1.0 & 1.6 & 1.5 & 1.4 \\
\hline Total $\ldots \ldots \ldots \ldots \ldots \ldots \ldots \ldots \ldots \ldots$ & 436.7 & 446.8 & 457.3 & 495.3 & 491.8 & 479.4 \\
\hline \multicolumn{7}{|l|}{ Total Carbon Emissions ${ }^{5}$} \\
\hline 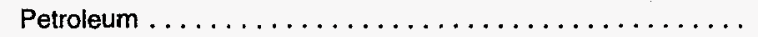 & 560.4 & 574.9 & 586.1 & 628.1 & 623.2 & 609.1 \\
\hline Natural Gas $\ldots \ldots \ldots \ldots \ldots \ldots \ldots$ & 257.4 & 258.8 & 264.8 & 285.7 & 281.1 & 275.3 \\
\hline Coal $\ldots \ldots \ldots \ldots \ldots \ldots \ldots \ldots \ldots \ldots$ & 66.0 & 67.4 & 66.5 & 75.9 & 65.3 & 60.2 \\
\hline Other ${ }^{4} \ldots \ldots \ldots \ldots \ldots \ldots \ldots \ldots \ldots \ldots \ldots \ldots \ldots \ldots$ & 0.0 & 0.0 & 0.0 & 0.0 & 0.0 & 0.0 \\
\hline 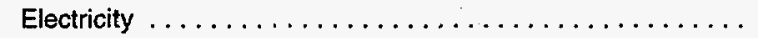 & 490.6 & 496.2 & 495.8 & 523.8 & 520.5 & 488.3 \\
\hline Total $\ldots \ldots \ldots \ldots \ldots \ldots \ldots \ldots \ldots \ldots$ & $1,374.4$ & $1,397.3$ & $1,413.2$ & $\mathbf{1 , 5 1 3 . 5}$ & $1,490.1$ & $1,432.9$ \\
\hline \multicolumn{7}{|l|}{ Electric Generators ${ }^{6}$} \\
\hline Petroleum $\ldots \ldots \ldots \ldots \ldots \ldots \ldots \ldots \ldots \ldots \ldots \ldots$ & 22.5 & 20.9 & 15.5 & 14.9 & 14.5 & 10.5 \\
\hline$\ldots \ldots \ldots \ldots \ldots \ldots \ldots \ldots$ & 39.5 & 44.9 & 47.3 & 47.7 & 45.8 & 31.6 \\
\hline$\ldots \ldots \ldots \ldots \ldots \ldots \ldots \ldots$ & 428.6 & 430.4 & 433.0 & 461.1 & 460.2 & 446.1 \\
\hline Total $\ldots \ldots \ldots \ldots \ldots \ldots \ldots \ldots \ldots \ldots$ & 490.6 & 496.2 & 495.8 & 523.8 & 520.5 & 488.3 \\
\hline \multicolumn{7}{|l|}{ Total Carbon Emissions ${ }^{7}$} \\
\hline Petroleum . . . . . . . . . & 582.9 & 595.8 & 601.6 & 643.1 & 637.7 & 619.6 \\
\hline Natural Gas $\ldots \ldots \ldots \ldots \ldots \ldots \ldots \ldots \ldots$ & 296.9 & 303.7 & 312.1 & 333.4 & 326.9 & 307.0 \\
\hline 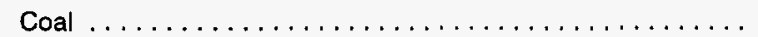 & 494.6 & 497.8 & 499.4 & 537.0 & 525.4 & 506.3 \\
\hline$\ldots \ldots \ldots \ldots \ldots \ldots \ldots$ & 0.0 & 0.0 & 0.0 & 0.0 & 0.0 & 0.0 \\
\hline Total $\ldots \ldots \ldots \ldots \ldots \ldots \ldots \ldots \ldots \ldots \ldots$ & $1,374.4$ & $1,397.3$ & $1,413.2$ & $1,513.5$ & $1,490.1$ & $1,432.9$ \\
\hline Carbon Emissions (metric tons per person) $\ldots \ldots \ldots \ldots \ldots$ & 5.3 & 5.4 & 5.4 & 5.5 & 5.4 & 5.2 \\
\hline
\end{tabular}

Includes consumption by cogenerators.

${ }^{2}$ Includes lease and plant fuel.

${ }^{3}$ Includes pipeline fuel natural gas and compressed natural gas used as vehicle fuel.

"Other includes methanol and liquid hydrogen.

sMeasured for delivered energy consumption.

Includes all electric power generators except cogenerators, which produce electricity as a by-product of other processes.

${ }^{7}$ Measured for total energy consumption, with emissions for electric power generators distributed to the primary fuels. 
Table B17. Carbon Emissions by End-Use Sector and Source (Continued) (Million Metric Tons per Year, Unless Otherwise Noted)

\begin{tabular}{c|c|c|c|c|c|c|c|c|c|c|c|}
\hline \multicolumn{9}{c|}{ Projections } & \multicolumn{2}{c}{$\begin{array}{c}\text { Annual Growth } \\
\text { 1994-2015 (percent) }\end{array}$} \\
\hline $\begin{array}{c}\text { Slow } \\
\text { Technology }\end{array}$ & $\begin{array}{c}2010 \\
\text { Reference }\end{array}$ & $\begin{array}{c}\text { Rapid } \\
\text { Technology }\end{array}$ & $\begin{array}{c}\text { Slow } \\
\text { Technology }\end{array}$ & Reference & $\begin{array}{c}\text { Rapid } \\
\text { Technology }\end{array}$ & $\begin{array}{c}\text { Slow } \\
\text { Technology }\end{array}$ & Reference & $\begin{array}{c}\text { Rapid } \\
\text { Technology }\end{array}$ & $\begin{array}{c}\text { Slow } \\
\text { Technology }\end{array}$ & $\begin{array}{c}\text { Rapid } \\
\text { Reference }\end{array}$ \\
\hline
\end{tabular}

\begin{tabular}{|c|c|c|c|c|c|c|c|c|c|c|c|}
\hline 24.3 & 23.5 & 23.0 & 23.9 & 22.8 & 22.0 & 24.0 & 22.5 & 21.3 & -0.3 & -0.6 & -0.8 \\
\hline 78.6 & 76.8 & 73.7 & 81.8 & 79.2 & 74.3 & 84.7 & 80.8 & 74.8 & 0.8 & 0.5 & 0.2 \\
\hline 1.3 & 1.2 & 1.1 & 1.3 & 1.2 & 1.0 & 1.3 & 1.1 & 0.9 & -0.4 & -1.2 & -2.2 \\
\hline 202.9 & 198.8 & 182.4 & 225.3 & 217.1 & 197.7 & 256.2 & 239.3 & 223.5 & 1.9 & 1.6 & 1.3 \\
\hline 307.1 & 300.4 & 280.3 & 332.3 & 320.3 & 295.0 & 366.2 & 343.8 & 320.5 & 1.5 & 1.2 & 0.8 \\
\hline 14.7 & 14.7 & 15.4 & 14.5 & 14.5 & 15.2 & 14.6 & 14.4 & 15.0 & 0.0 & -0.1 & 0.1 \\
\hline 47.7 & 47.6 & 45.5 & 48.9 & 49.2 & 46.7 & 49.5 & 50.6 & 48.3 & 0.6 & 0.7 & 0.5 \\
\hline 2.3 & 2.3 & 2.3 & 2.4 & 2.4 & 2.4 & 2.5 & 2.5 & 2.5 & 0.9 & 0.9 & 0.9 \\
\hline 178.7 & 176.2 & 153.1 & 190.5 & 187.1 & 160.5 & 208.1 & 200.6 & 174.3 & 1.4 & 1.2 & 0.5 \\
\hline 243.5 & 240.8 & 216.3 & 256.4 & 253.3 & 224.9 & 274.7 & 268.1 & 240.1 & 1.2 & 1.0 & 0.5 \\
\hline 110.0 & 107.3 & 98.4 & 114.8 & 109.5 & 91.8 & 123.6 & 113.0 & 88.4 & 1.1 & 0.6 & -0.5 \\
\hline 163.2 & 158.3 & 152.1 & 167.3 & 163.9 & 154.0 & 166.2 & 167.6 & 150.7 & 1.0 & 1.1 & 0.6 \\
\hline 78.7 & 60.4 & 49.4 & 89.1 & 60.2 & 44.4 & 102.5 & 61.8 & 41.4 & 2.3 & -0.2 & -2.0 \\
\hline 195.2 & 191.9 & 176.4 & 205.8 & 200.5 & 180.5 & 219.5 & 209.1 & 188.0 & 1.3 & 1.0 & 0.5 \\
\hline 547.1 & 518.0 & 476.4 & 577.0 & 534.1 & 470.7 & 611.8 & 551.6 & 468.5 & 1.3 & 0.8 & 0.0 \\
\hline 524.5 & 511.3 & 485.9 & 557.2 & 532.7 & 491.1 & 583.8 & 547.2 & 489.5 & 1.4 & 1.1 & 0.6 \\
\hline 10.7 & 10.6 & 9.7 & 13.1 & 12.7 & 11.3 & 15.8 & 15.5 & 13.7 & 2.4 & 2.3 & 1.7 \\
\hline 0.7 & 0.7 & 0.7 & 1.7 & 1.6 & 1.5 & 2.5 & 2.3 & 2.1 & 24.1 & 23.7 & 23.2 \\
\hline 3.6 & 3.4 & 3.0 & 6.0 & 5.5 & 4.8 & 7.8 & 6.7 & 5.9 & 10.2 & 9.4 & 8.8 \\
\hline 539.5 & 526.0 & 499.3 & 578.0 & 552.4 & 508.6 & 609.9 & 571.7 & 511.3 & 1.5 & 1.2 & 0.6 \\
\hline 673.5 & 656.8 & 622.8 & 710.4 & 679.6 & 620.1 & 746.0 & 697.1 & 614.1 & 1.2 & 0.9 & 0.3 \\
\hline 300.3 & 293.4 & 281.0 & 311.1 & 305.0 & 286.4 & 316.2 & 314.5 & 287.5 & 1.0 & 0.9 & 0.5 \\
\hline 82.4 & 64.0 & 52.9 & 92.9 & 63.8 & 47.8 & 106.4 & 65.5 & 44.8 & 2.2 & -0.1 & -1.9 \\
\hline 0.7 & 0.7 & 0.7 & 1.7 & 1.6 & 1.5 & 2.5 & 2.3 & 2.1 & 24.1 & 23.7 & 23.2 \\
\hline 580.3 & 570.3 & 514.9 & 627.6 & 610.2 & 543.5 & 691.5 & 655.7 & 591.8 & 1.6 & 1.3 & 0.8 \\
\hline $1,637.3$ & $1,585.2$ & $1,472.3$ & $1,743.7$ & $1,660.0$ & $1,499.2$ & $1,862.6$ & $1,735.1$ & $1,540.4$ & 1.4 & 1.0 & 0.5 \\
\hline 579.0 & 555.0 & 519.9 & 610.6 & 573.1 & 529.9 & 661.5 & 591.4 & 540.6 & 1.4 & 0.8 & 0.4 \\
\hline 0.7 & 0.7 & 0.7 & 1.7 & 1.6 & 1.5 & 2.5 & 2.3 & 2.1 & 24.1 & 23.7 & 23.2 \\
\hline $1,637.3$ & $1,585.2$ & $1,472.3$ & $1,743.7$ & $1,660.0$ & $1,499.2$ & $1,862.6$ & $1,735.1$ & $1,540.4$ & 1.4 & 1.0 & 0.5 \\
\hline 5.7 & 5.5 & 5.1 & 5.8 & 5.6 & 5.0 & 6.0 & 5.6 & 5.0 & 0.5 & 0.2 & -0.4 \\
\hline
\end{tabular}

Note: Totals may not equal sum of components due to independent rounding.

Sources: Utility coal carbon emissions from Energy Information Administration (EIA), Emissions of Greenhouse Gases in the United States, 1987-1992, DOE/EIA-0573

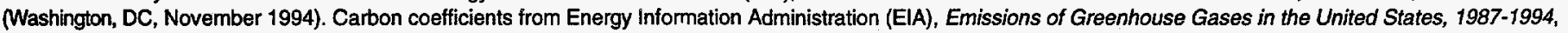
DOE/EIA-0573(95) (Washington, DC, October 1995). 1993 consumption estimates based on: ElA, State Energy Data Report 1993, DOE/EIA-0214(93) (Washington, DC, July 1995). 1994 consumption estimate based on: EIA, Short Term Energy Out/ook, DOE/EIA-0202(95/3Q) (Washington, DC, August 1995). Consumption

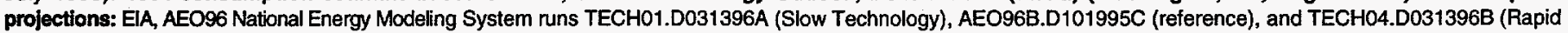
Technology). 
Table B18. Macroeconomic Indicators

(Billion 1987 Dollars, Unless Otherwise Noted)

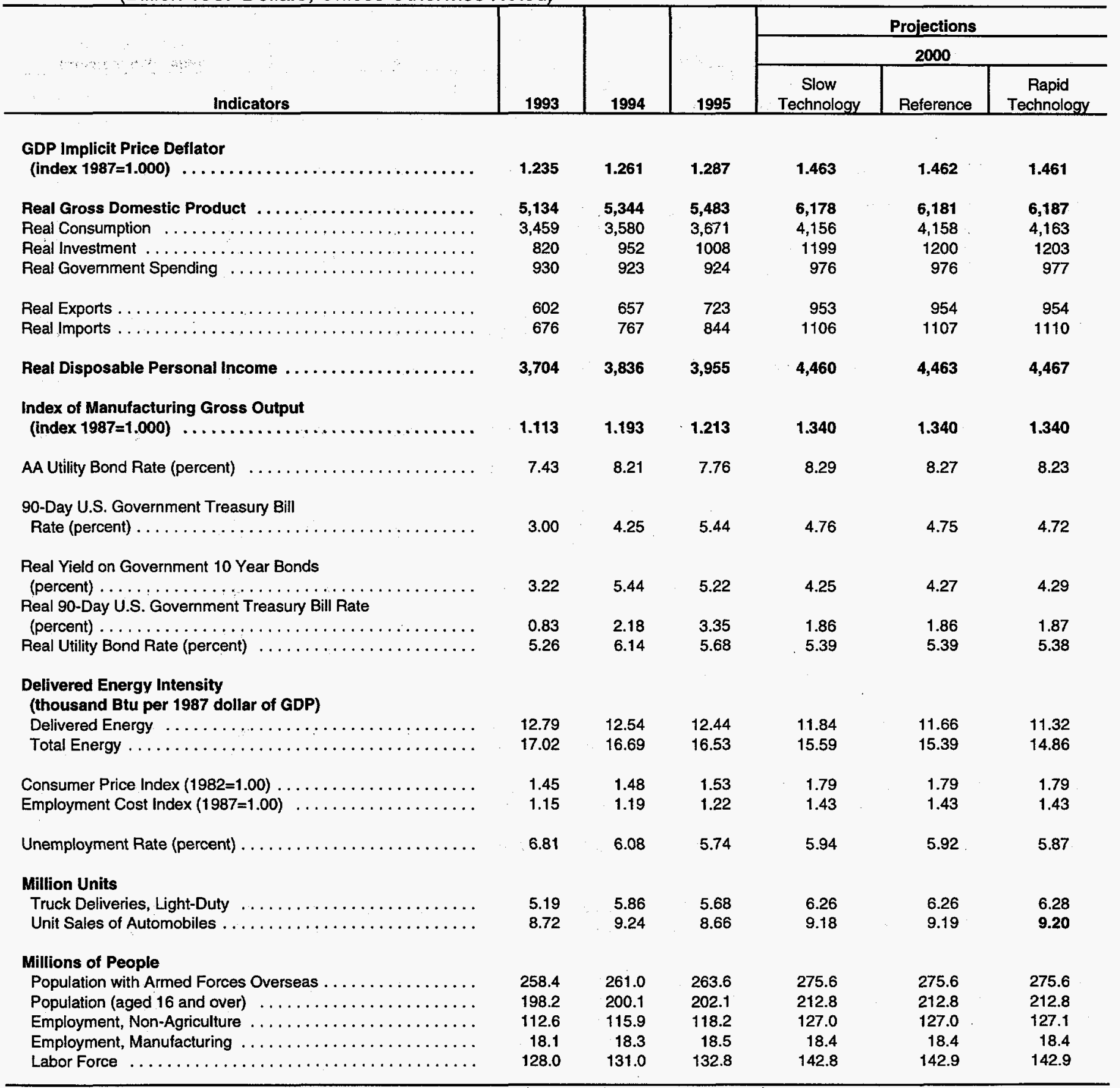


Table B18. Macroeconomic Indicators (Continued) (Billion 1987 Dollars, Unless Otherwise Noted)

\begin{tabular}{|c|c|c|c|c|c|c|c|c|c|c|c|}
\hline \multicolumn{9}{|c|}{ Projections } & \multirow{2}{*}{\multicolumn{3}{|c|}{$\begin{array}{c}\text { Annual Growth } \\
1994-2015 \text { (percent) }\end{array}$}} \\
\hline \multicolumn{3}{|c|}{2005} & \multicolumn{3}{|c|}{2010} & \multicolumn{3}{|c|}{2015} & & & \\
\hline $\begin{array}{c}\text { Slow } \\
\text { Technology }\end{array}$ & Relerence & $\begin{array}{c}\text { Rapid } \\
\text { Technology }\end{array}$ & $\begin{array}{c}\text { Slow } \\
\text { Technology }\end{array}$ & Reference & $\begin{array}{c}\text { Rapid } \\
\text { Technology }\end{array}$ & $\begin{array}{c}\text { Slow } \\
\text { Technology }\end{array}$ & Reference & $\begin{array}{c}\text { Rapid } \\
\text { Technology }\end{array}$ & $\begin{array}{c}\text { Slow } \\
\text { Technology }\end{array}$ & Reference & $\begin{array}{c}\text { Rapid } \\
\text { Technology }\end{array}$ \\
\hline 1.726 & 1.723 & 1.718 & 2.054 & 2.047 & 2.037 & 2.448 & 2.433 & 2.418 & 3.2 & 3.2 & 3.2 \\
\hline 6,893 & 6,901 & 6,919 & 7,501 & 7523 & 7,552 & 8,072 & 8,114 & 8,158 & 2.0 & 2.0 & 2.0 \\
\hline 4,596 & 4,603 & 4,618 & 4,980 & 4,999 & 5,024 & 5,365 & 5,401 & 5,441 & 1.9 & 2.0 & 2.0 \\
\hline 1,416 & 1,419 & 1,426 & 1,587 & 1,595 & 1,605 & 1,728 & 1,744 & 1,761 & 2.9 & 2.9 & 3.0 \\
\hline 1,030 & 1,031 & 1,034 & 1,085 & 1,089 & 1,094 & 1,136 & 1,143 & 1,152 & 1.0 & 1.0 & 1.1 \\
\hline 1,271 & 1,273 & 1,276 & 1,638 & 1,643 & 1,651 & 2,010 & 2,022 & 2,037 & 5.5 & 5.5 & 5.5 \\
\hline 1,421 & 1,426 & 1,435 & 1,790 & 1,803 & 1,823 & 2,166 & 2,197 & 2,233 & 5.1 & 5.1 & 5.2 \\
\hline 4,950 & 4,958 & 4,973 & 5,363 & 5383 & 5,408 & 5,761 & 5,798 & 5,837 & 2.0 & 2.0 & 2.0 \\
\hline 1.503 & 1.504 & 1.504 & 1.632 & 1.634 & 1.635 & 1.761 & 1.766 & 1.769 & 1.9 & 1.9 & 1.9 \\
\hline 8.62 & 8.57 & 8.47 & 8.35 & 8.24 & 8.11 & 8.13 & 7.96 & 7.78 & N/A & $N / A$ & $N / A$ \\
\hline 4.88 & 4.84 & 4.77 & 4.73 & 4.66 & 4.57 & 4.59 & 4.49 & 4.40 & N/A & N/A & N/A \\
\hline 3.73 & 3.72 & 3.71 & 3.31 & 3.28 & 3.19 & 3.41 & 3.32 & 3.15 & N/A & N/A & N/A \\
\hline 1.19 & 1.18 & 1.15 & 1.19 & 1.17 & 1.11 & 1.03 & 0.97 & 0.90 & $N / A$ & $N / A$ & N/A \\
\hline 4.94 & 4.91 & 4.85 & 4.81 & 4.76 & 4.65 & 4.57 & 4.44 & 4.28 & N/A & $N / A$ & N/A \\
\hline 11.35 & 11.01 & 10.33 & 11.04 & 10.53 & 9.52 & 10.80 & 10.15 & 8.83 & -0.7 & -1.0 & -1.7 \\
\hline 14.96 & 14.55 & 13.57 & 14.52 & 13.92 & 12.58 & 14.10 & 13.32 & 11.71 & -0.8 & -1.1 & -1.7 \\
\hline 2.17 & 2.16 & 2.15 & 2.65 & 2.63 & 2.61 & 3.23 & 3.19 & 3.13 & 3.8 & 3.7 & 3.6 \\
\hline 1.73 & 1.73 & 1.72 & 2.11 & 2.11 & 2.10 & 2.59 & 2.57 & 2.55 & 3.8 & 3.7 & 3.7 \\
\hline 5.83 & 5.79 & 5.69 & 6.25 & 6.15 & 6.05 & 6.21 & 6.05 & 5.91 & $\mathbf{N} / \mathbf{A}$ & N/A & N/A \\
\hline 7.09 & 7.12 & 7.16 & 7.70 & 7.76 & 7.83 & 8.29 & 8.40 & 8.51 & 1.7 & 1.7 & 1.8 \\
\hline 9.32 & 9.33 & 9.34 & 9.20 & 9.22 & 9.23 & 9.13 & 9.15 & 9.15 & -0.1 & 0.0 & 0.0 \\
\hline 287.1 & 287.1 & 287.1 & 298.9 & 298.9 & 298.9 & 311.2 & 311.2 & 311.2 & 0.8 & 0.8 & 0.8 \\
\hline 223.8 & 223.8 & 223.8 & 235.4 & 235.4 & 235.4 & 245.8 & 245.8 & 245.8 & 1.0 & 1.0 & 1.0 \\
\hline 136.5 & 136.6 & 136.7 & 142.4 & 142.7 & 143.0 & 146.8 & 147.4 & 147.8 & 1.1 & 1.2 & 1.2 \\
\hline 18.7 & 18.7 & 18.7 & 18.4 & 18.4 & 18.5 & 17.9 & 18.0 & 18.1 & -0.1 & -0.1 & -0.1 \\
\hline 152.1 & 152.2 & 152.3 & 159.3 & 159.4 & 159.5 & 163.1 & 163.3 & 163.4 & 1.0 & 1.1 & 1.1 \\
\hline
\end{tabular}

GDP = Gross domestic product.

Btu $=$ British thermal unit.

N/A $=$ Not available.

Note: Totals may not equal sum of components due to independent rounding.

Sources: 1993 and 1994: Data Resources Incorporated (DRl), DRI Trend0295. Projections: Energy Information Administration, AEO96 National Energy Modeling System runs TECH01.D031396A (Slow Technology), AEO96B.D101995C (reference), and TECH04.D031396B (Rapid Technology). 
Table B19. International Petroleum Supply and Disposition Summary (Million Barrels per Day, Unless Otherwise Noted)

\begin{tabular}{|c|c|c|c|c|c|c|}
\hline \multirow[b]{3}{*}{ Supply and Disposition } & \multirow[b]{3}{*}{1993} & \multirow[b]{3}{*}{1994} & \multirow[b]{3}{*}{1995} & \multicolumn{3}{|c|}{ Projections } \\
\hline & & & & \multicolumn{3}{|c|}{2000} \\
\hline & & & & $\begin{array}{c}\text { Slow } \\
\text { Technology }\end{array}$ & Reference & $\begin{array}{c}\text { Rapid } \\
\text { Technology }\end{array}$ \\
\hline World Oil Price (1994 dollars per barrel) ${ }^{1} \ldots \ldots \ldots \ldots \ldots \ldots$ & 16.48 & 15.52 & 16.82 & 19.66 & 19.27 & 18.48 \\
\hline \multicolumn{7}{|l|}{ Production ${ }^{2}$} \\
\hline \multicolumn{7}{|l|}{ OECD } \\
\hline U.S. (50 states) $\ldots \ldots \ldots \ldots \ldots \ldots \ldots \ldots \ldots \ldots \ldots \ldots$ & 9.54 & 9.34 & 9.35 & 8.45 & 8.54 & 8.55 \\
\hline 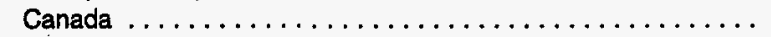 & 2.24 & 2.33 & 2.42 & 2.48 & 2.48 & 2.48 \\
\hline Mexico $\ldots \ldots \ldots \ldots \ldots \ldots \ldots \ldots \ldots \ldots$ & 3.16 & 3.18 & 3.18 & 3.21 & 3.21 & 3.20 \\
\hline OECD Europe ${ }^{3} \ldots \ldots \ldots \ldots \ldots \ldots \ldots \ldots \ldots$ & 5.29 & 6.13 & 6.46 & 6.82 & 6.82 & 6.82 \\
\hline Other OECD $\ldots \ldots \ldots \ldots \ldots \ldots \ldots \ldots \ldots \ldots \ldots$ & 0.68 & 0.73 & 0.75 & 0.76 & 0.76 & 0.75 \\
\hline Total OECD $\ldots \ldots \ldots \ldots \ldots \ldots \ldots \ldots \ldots \ldots \ldots \ldots$ & 20.90 & 21.70 & 22.16 & 21.72 & 21.80 & 21.79 \\
\hline \multicolumn{7}{|l|}{ Developing Countries } \\
\hline Other South \& Central America $\ldots \ldots \ldots \ldots \ldots \ldots \ldots$ & 2.26 & 2.82 & 2.91 & 2.94 & 2.94 & 2.93 \\
\hline 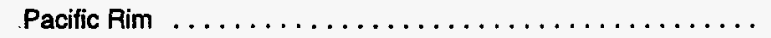 & 1.81 & 1.89 & 1.95 & 2.13 & 2.12 & 2.12 \\
\hline OPEC $\ldots \ldots \ldots \ldots \ldots \ldots \ldots \ldots \ldots \ldots \ldots$ & 27.57 & 27.63 & 27.63 & 34.77 & 34.67 & 34.39 \\
\hline Other Developing Countries $\ldots \ldots \ldots \ldots \ldots \ldots \ldots \ldots$ & 3.84 & 3.82 & 3.93 & 4.02 & 4.02 & 4.01 \\
\hline Total Developing Countries . . . . . . . . . . . . . . & $\mathbf{3 5 . 4 8}$ & 36.16 & 36.43 & 43.86 & 43.76 & 43.46 \\
\hline \multicolumn{7}{|l|}{ Eurasia } \\
\hline Former Soviet Union $\ldots \ldots \ldots \ldots \ldots \ldots \ldots \ldots$ & 8.10 & 7.01 & 6.60 & 7.30 & 7.29 & 7.28 \\
\hline Eastern Europe $\ldots \ldots \ldots \ldots \ldots \ldots \ldots \ldots \ldots \ldots \ldots$ & 0.27 & 0.30 & 0.29 & 0.27 & 0.27 & 0.27 \\
\hline 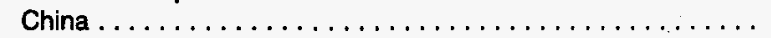 & 2.91 & 2.94 & 2.97 & 3.02 & 3.02 & 3.02 \\
\hline Total Eurasia $\ldots \ldots \ldots \ldots \ldots \ldots \ldots \ldots \ldots \ldots$ & 11.28 & 10.25 & 9.86 & 10.59 & 10.58 & 10.57 \\
\hline Total Production $\ldots \ldots \ldots \ldots \ldots \ldots \ldots \ldots \ldots$ & 67.66 & 68.11 & 68.45 & 76.17 & 76.14 & 75.82 \\
\hline \multicolumn{7}{|l|}{ Consumption } \\
\hline \multicolumn{7}{|l|}{ OECD } \\
\hline U.S. (50 states) $\ldots \ldots \ldots \ldots \ldots \ldots \ldots \ldots \ldots \ldots \ldots$ & 17.24 & 17.72 & 17.79 & 18.91 & 18.79 & 18.27 \\
\hline 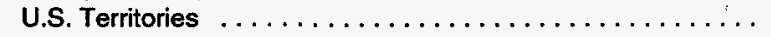 & 0.24 & 0.26 & 0.26 & 0.30 & 0.30 & 0.30 \\
\hline Canada $\ldots \ldots \ldots \ldots \ldots \ldots \ldots \ldots \ldots \ldots \ldots$ & 1.69 & 1.73 & 1.76 & 1.86 & 1.86 & 1.87 \\
\hline Mexico $\ldots \ldots \ldots \ldots \ldots \ldots \ldots \ldots \ldots \ldots$ & 1.87 & 1.91 & 1.94 & 2.06 & 2.06 & 2.07 \\
\hline Japan $\ldots \ldots \ldots \ldots \ldots \ldots \ldots \ldots \ldots \ldots$ & 5.41 & 5.68 & 5.79 & 6.34 & 6.36 & 6.40 \\
\hline Australia and New Zealand. $\ldots \ldots \ldots \ldots \ldots \ldots \ldots \ldots$ & 0.88 & 0.94 & 0.94 & 1.02 & 1.02 & 1.02 \\
\hline OECD Europe ${ }^{3} \ldots \ldots \ldots \ldots \ldots \ldots \ldots \ldots \ldots \ldots$ & 13.50 & 13.59 & 13.78 & 14.21 & 14.24 & 14.29 \\
\hline Total OECD $\ldots \ldots \ldots \ldots \ldots \ldots \ldots \ldots \ldots \ldots$ & 40.82 & 41.82 & 42.26 & 44.70 & 44.63 & 44.23 \\
\hline \multicolumn{7}{|l|}{ Developing Countries } \\
\hline Other South and Central America . . . . . . & 3.20 & 3.26 & 3.34 & 3.93 & 3.93 & 3.94 \\
\hline Pacific $\operatorname{Rim} \ldots \ldots \ldots \ldots \ldots \ldots \ldots$ & 3.87 & 4.12 & 4.39 & 7.17 & 7.17 & 7.19 \\
\hline 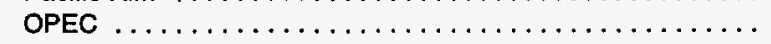 & 4.58 & 4.74 & 4.94 & 5.56 & 5.56 & 5.56 \\
\hline Other Developing Countries $\ldots \ldots \ldots \ldots \ldots \ldots \ldots \ldots \ldots$ & 4.10 & 4.21 & 4.30 & 4.77 & 4.78 & 4.79 \\
\hline Total Developing Countries . . . . . . . . . . . . . . & 15.76 & 16.33 & 16.98 & 21.42 & 21.45 & 21.49 \\
\hline
\end{tabular}


Table B19. International Petroleum Supply and Disposition Summary (Continued) (Million Barrels per Day, Unless Otherwise Noted)

\begin{tabular}{|c|c|c|c|c|c|c|c|c|c|c|c|}
\hline \multicolumn{9}{|c|}{ Projections } & \multirow{2}{*}{\multicolumn{3}{|c|}{$\begin{array}{c}\text { Annual Growth } \\
1994-2015 \text { (percent) }\end{array}$}} \\
\hline \multicolumn{3}{|c|}{2005} & \multicolumn{3}{|c|}{2010} & \multicolumn{3}{|c|}{2015} & & & \\
\hline $\begin{array}{c}\text { Slow } \\
\text { Technology }\end{array}$ & Reference & $\begin{array}{c}\text { Rapid } \\
\text { Technology }\end{array}$ & $\begin{array}{c}\text { Slow } \\
\text { Technology }\end{array}$ & Reference & $\begin{array}{c}\text { Rapid } \\
\text { Technology }\end{array}$ & $\begin{array}{c}\text { Slow } \\
\text { Technology }\end{array}$ & Reference & {$\left[\begin{array}{c}\text { Rapid } \\
\text { Technology }\end{array}\right.$} & $\begin{array}{c}\text { Slow } \\
\text { Technology }\end{array}$ & Reference & $\begin{array}{c}\text { Rapid } \\
\text { Technology }\end{array}$ \\
\hline 23.00 & 21.87 & 19.77 & 25.60 & 23.71 & 20.55 & 28.58 & 25.43 & 21.18 & 3.0 & 2.4 & 1.5 \\
\hline 8.23 & 8.38 & 8.36 & 8.60 & 8.79 & 8.61 & 9.01 & 9.38 & 9.25 & -0.2 & 0.0 & 0.0 \\
\hline 2.48 & 2.47 & 2.47 & 2.37 & 2.37 & 2.35 & 2.27 & 2.26 & 2.24 & -0.1 & -0.1 & -0.2 \\
\hline 3.17 & 3.16 & 3.14 & 3.12 & 3.11 & 3.07 & 3.08 & 3.06 & 3.01 & -0.1 & -0.2 & -0.3 \\
\hline 6.08 & 6.07 & 6.05 & 5.25 & 5.24 & 5.21 & 4.31 & 4.29 & 4.25 & -1.7 & -1.7 & -1.7 \\
\hline 0.72 & 0.71 & 0.71 & 0.67 & 0.66 & 0.65 & 0.62 & 0.61 & 0.60 & -0.7 & -0.8 & -0.9 \\
\hline 20.67 & 20.80 & 20.72 & 20.02 & 20.16 & 19.89 & 19.29 & 19.60 & 19.35 & -0.6 & -0.5 & -0.5 \\
\hline 2.70 & 2.69 & 2.68 & 2.37 & 2.36 & 2.33 & 1.95 & 1.94 & 1.91 & -1.7 & -1.8 & -1.8 \\
\hline 2.28 & 2.28 & 2.26 & 2.21 & 2.20 & 2.17 & 2.07 & 2.05 & 2.02 & 0.4 & 0.4 & 0.3 \\
\hline 41.34 & 41.16 & 40.87 & 46.70 & 46.55 & 46.28 & 52.80 & 52.37 & 52.12 & 3.1 & 3.1 & 3.1 \\
\hline 3.88 & 3.87 & 3.84 & 3.61 & 3.59 & 3.55 & 3.25 & 3.22 & 3.17 & -0.8 & -0.8 & -0.9 \\
\hline 50.20 & 50.00 & 49.65 & 54.88 & 54.70 & 54.33 & 60.07 & 59.58 & 59.22 & 2.4 & 2.4 & 2.4 \\
\hline 8.43 & 8.41 & 8.36 & 9.61 & 9.55 & 9.45 & 10.36 & 10.27 & 10.12 & 1.9 & 1.8 & 1.8 \\
\hline 0.24 & 0.23 & 0.23 & 0.22 & 0.21 & 0.21 & 0.19 & 0.18 & 0.18 & -2.3 & -2.3 & -2.4 \\
\hline 3.08 & 3.07 & 3.05 & 3.06 & 3.04 & 3.01 & 3.02 & 3.00 & 2.95 & 0.1 & 0.1 & 0.0 \\
\hline 11.75 & 11.71 & 11.64 & 12.88 & 12.81 & 12.67 & 13.58 & 13.46 & 13.26 & 1.3 & 1.3 & 1.2 \\
\hline 82.62 & 82.51 & 82.01 & 87.79 & 87.67 & 86.90 & 92.94 & 92.63 & 91.83 & 1.5 & 1.5 & 1.4 \\
\hline 20.37 & 19.89 & 18.63 & 21.52 & 20.67 & 18.47 & 22.71 & 21.18 & 18.20 & 1.2 & 0.9 & 0.1 \\
\hline 0.33 & 0.33 & 0.34 & 0.35 & 0.36 & 0.38 & 0.37 & 0.39 & 0.41 & 1.8 & 2.0 & 2.3 \\
\hline 1.93 & 1.95 & 2.00 & 1.99 & 2.04 & 2.12 & 2.06 & 2.13 & 2.26 & 0.8 & 1.0 & 1.3 \\
\hline 2.22 & 2.24 & 2.27 & 2.38 & 2.41 & 2.49 & $2.5 t$ & 2.57 & 2.69 & 1.3 & 1.4 & 1.6 \\
\hline 6.56 & 6.64 & 6.81 & 6.68 & 6.85 & 7.20 & 6.79 & 7.08 & 7.65 & 0.9 & 1.1 & 1.4 \\
\hline 1.08 & 1.08 & 1.10 & 1.14 & 1.15 & 1.17 & 1.20 & 1.21 & 1.24 & 1.2 & 1.2 & 1.3 \\
\hline 14.48 & 14.57 & 14.74 & 14.60 & 14.75 & 15.04 & 14.69 & 14.92 & 15.32 & 0.4 & 0.4 & 0.6 \\
\hline 46.97 & 46.71 & 45.90 & 48.65 & 48.22 & 46.86 & 50.33 & 49.49 & 47.77 & 0.9 & 0.8 & 0.6 \\
\hline 4.26 & 4.27 & 4.30 & 4.53 & 4.56 & 4.61 & 4.79 & 4.84 & 4.92 & 1.9 & 1.9 & 2.0 \\
\hline 8.74 & 8.77 & 8.84 & 9.62 & 9.69 & 9.82 & 10.45 & 10.57 & 10.76 & 4.5 & 4.6 & 4.7 \\
\hline 6.14 & 6.14 & 6.14 & 6.78 & 6.78 & 6.78 & 7.48 & 7.48 & 7.48 & 2.2 & 2.2 & 2.2 \\
\hline 5.10 & 5.13 & 5.20 & 5.40 & 5.48 & 5.64 & 5.73 & 5.87 & 6.14 & 1.5 & 1.6 & 1.8 \\
\hline 24.23 & 24.31 & 24.49 & 26.33 & 26.51 & 26.85 & 28.45 & 28.75 & 29.29 & 2.7 & 2.7 & 2.8 \\
\hline
\end{tabular}

'Average reliner acquisition cost of imported crude oil.

${ }^{2}$ Includes production of crude oil (including lease condensates), natural gas plant liquids, other hydrogen and hydrocarbons for refinery feedstocks, alcohol, liquids produced from coal and other sources, and refinery gains.

'OECD Europe includes the unified Germany.

OECD = Organization for Economic Cooperation and Development - Australia, Austria, Belgium, Canada, Denmark, Finland, France, Germany, Greece, Iceland, Ireland,

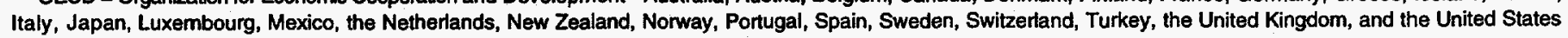
(including territories).

Pacific Rim = Hong Kong, Malaysia, Philippines, Singapore, South Korea, Taiwan, and Thailand.

OPEC = Organization of Petroleum Exporting Countries - Algeria, Gabon, Indonesia, Iran, Iraq, Kuwait, Libya, Nigeria, Qatar, Saudi Arabia, the United Arab Emirates, and Venezuela.

Eurasia = Albania, Bulgaria, China, Czech Republic, Hungary, Poland, Romania, Slovak Republic, the Former Soviet Union, and the Former Yugoslavia.

N/A $=$ Not applicable.

Note: Totals may not equal sum of components due to independent rounding.

Sources: 1993 to 1995: Energy Information Administration (EIA), Short-Term Energy Outlook, DOE/EIA-0202(95/3Q) (Washington, DC, August 1995). Projections: EIA, AEO96 National Energy Modeling System nuns TECH01.D031396A (Slow Technology), AEO96B.D101995C (reference), and TECH04.D031396B (Rapid Technology). 



\title{
The Outlook for U.S. Import Dependence
}

\author{
by Bruce H. Bawks
}

\section{Introduction}

Projections of petroleum (crude and product) import dependence in the Annual Energy Outlook $1996^{1}$ (AEO96) are slightly lower than the previous year's projections. In 2010, AEO96 estimates 57 percent of consumption ${ }^{2}$ will be met by net imports of crude oil and petroleum products, compared to the 59 percent estimate from the Annual Energy Outlook 1995 $5^{3}$ (AEO95). Despite somewhat lower prices in AEO96, consumption in 2010 shows a small decline and production a slight increase ${ }^{4}$ compared to $A E O 95$, resulting in the lower import estimates. Over the past several $A E O$ s, world oil price projections have fallen, yet the forecasts for petroleum imports have remained essentially the same. The lower prices have not led to substantial increases in forecasts for consumption nor to significant declines in production estimates, primarily as a result of assumed technology and efficiency improvements.

Nevertheless, import dependence (measured based on net imports of crude oil and petroleum products from all countries) is projected to rise beyond historic peaks over the next several years. Consumption increases, which propelled the rise in imports in the $1970 \mathrm{~s},{ }^{5}$ and production decreases, which led to higher import levels in recent years, are combining to push import dependence to new levels. In the later years of the forecast, however, consumption increases are expected to slow and crude production is expected to rise, resulting in a small decline (about 2 percentage points) in import dependence.

Even though import dependence is rising to new levels, a shift in sources of supply has taken place since the 1970s, and this is expected to continue. Imports from Persian Gulf supply sources are expected to increase; however, greater amounts of petroleum are also coming from sources closer to the United States such as Canada and Mexico. This greater diversity of supply should be taken into account in measuring import dependence. This article will explore some issues related to measuring import dependence, provide an overview of historical import trends and the major factors affecting those trends, and give an assessment of projected trends in import dependence. This article is primarily descriptive and does not endorse policy alternatives or measure the benefits and costs of petroleum imports.

\section{Background}

The increasing dependence of the United States on imported petroleum became apparent in the 1970s and early 1980s, when first an oil embargo and then a war in the Middle East sent prices skyrocketing. The higher prices induced searches for other sources of crude supplies, switching to non-petroleum fuels, and research and development of alternative fuels. When the country developed a national energy strategy several years ago, the security of supplies was a key element of the strategy, with the concept of security as the antithesis of dependence. The invasion of Kuwait in 1990 again brought the issue of imported petroleum to the forefront.

Import dependence is important to measure because it provides the policy-maker and the analyst with some of the information needed to determine the Nation's vulnerability to disruptions in supply. It is important to remember, however, that importing petroleum may not be detrimental in and of itself. Japan, for example, has imported nearly all of its crude oil for decades, while making great strides economically. In this country, foreign oil has provided a

\footnotetext{
${ }^{1}$ Energy Information Administration, Annual Energy Outlook 1996, DOE/EIA-0383(96) (Washington, DC, January 1996).

${ }^{2}$ In this article, consumption is represented by product supplied, an approximation of consumption that is calculated by measuring the disappearance of products from primary supply sources.

${ }^{3}$ Energy Information Administration, Annual Energy Outlook 1995, DOE/EIA-0383(95) (Washington, DC, January 1995).

${ }^{4}$ The higher production is due primarily to increases in offshore production where technological improvements are expected to have the greatest effect.

${ }^{5}$ The lifting of import quotas in 1973 allowed more imports into the country and forced U.S. producers to compete against cheaper foreign imports.
} 
cheaper source of petroleum than could be produced domestically. The benefits of cheaper imported petroleum have to be weighed against the costs of possible disruptions in supply and the costs of attaining self-sufficiency.

Imports bridge the gap between domestic supplies, consisting primarily of crude oil production, and the consumption of petroleum products. Declining production and/or rising consumption increase(s) imports, other things being equal. Import reduction therefore requires either increases in domestic production or decreases in consumption. Policies to reduce import dependence must affect at least one of these areas. On the supply side, this can take the form of incentives to raise domestic crude oil production or the addition of other liquids such as oxygenates. On the consumption side, incentives can be provided to switch to other domestic energy sources, such as natural gas or alternative fuels, or to improve efficiency through technological changes or regulations such as the Corporate Average Fuel Economy (CAFE) standards. Disincentives such as taxes can also be used to reduce or slow the increase in consumption.

A related issue is the refining capability of the United States. Petroleum can be imported in the form of either crude oil or products. Currently, the majority of imports is crude oil. However, relatively small increases in domestic refining capacity are projected, and product imports are expected to grow. ${ }^{6}$ While this has only a small impact on import dependence calculations, the health of the domestic refining industry is an important part of the import dependence debate. The growth of product imports implies an increasing dependence not only on foreign crude oil but also on foreign refining centers to process the crude oil into the products that the country needs.

\section{Measures of Dependence}

Import dependence is typically measured in terms of the percentage of consumption that is met by imports. One question that arises is whether the imports side of the calculation should be total or net imports (net = imports exports). Some analysts and organizations, such as the American Petroleum Institute, report import dependence in terms of total imports. The Energy Information Admin- istration (EIA), on the other hand, uses net imports in its analyses and forecasts." The use of total imports tends to overstate dependence because it ignores the mitigating influence of product exports on dependence. With total imports in the numerator, comparisons in dependence over a number of years can also be substantially distorted due to changes in export patterns. ${ }^{8}$ EIA believes that the net imports definition gives a clearer indication of the fraction of oil consumed that could not have been supplied from domestic sources and is thus the most appropriate measure.

One criticism of the use of net imports, however, is that it understates dependence. Net imports of one product can be offset by net exports of another product that is not directly interchangeable. For example, net exports of petroleum coke reduce the dependence measure even though coke is not directly interchangeable with lighter petroleum products. Thus, in this sense, the net imports measure understates dependence because the net exports of one product cannot readily substitute for the net imports of the other product. ${ }^{10}$

One response to this criticism is to include only those products for which the country is a net importer and to ignore the net exports. This, however, does not have a large impact on the dependence calculation. The net positive imports measure typically exceeds that of total net imports by 1 to 2 percentage points. The difficulty of this calculation is the additional import and export data required on all the products. Furthermore, over a longer period of time, changes in refinery operations on the supply side along with fuel switching on the demand side can reduce the inability to readily substitute products.

Another issue related to measuring import dependence is the sources of supply. Imports from Canada, Mexico, and the Caribbean area, for example, can arrive via overland supply routes in some cases or across relatively short stretches of water. Thus, they are deemed to be more secure than imports from distant sources such as the Persian Gulf. Changes in sources of supply compared to the 1970s are expected to provide a greater diversity of supply which needs to be accounted for in measures of import dependence. This will be examined in more detail later in this paper.

${ }^{6}$ Energy Information Administration, Annual Energy Outlook 1996, DOE/EIA-0383(96) (Washington, DC, January 1996), pp. 49, 102, 103.

${ }^{7}$ C. William Skinner, "Measuring Dependence on Imported Oil," Monthly Energy Review, DOE/EIA-0035(95/08) (Washington, DC, August 1995), p.I.

${ }^{8}$ Ibid., p. ii.

${ }^{9}$ Tbid., p. I.

${ }^{10}$ Problems can also arise in substituting exports from one region for imports in another region. However, even these logistical problems can, with some difficulty, be overcome. 
Figure 1. Components of Petroleum Supply, 1950-2015

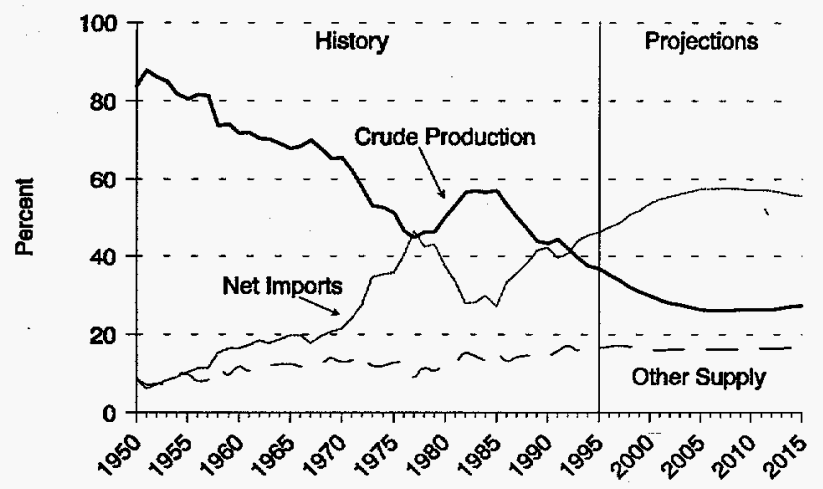

Source: Percentages calculated from the following data: History: 1950-1993: Energy Information Administration, Annual Energy Review 1994, DOE/EIA-0384(94) (Washington, DC, July 1995). 1994: Energy Information Administration, Petroleum Supply Annual 1994, DOE/EIA-0340(94)/1 (Washington, DC, May 1995). Projections: AEO96 National Energy Modeling System run AEO96B.D101995C.

\section{U.S. Import Trends}

Before looking more closely at the trends that are expected to affect petroleum imports in the future, we provide an historical perspective on major supply and demand trends that influenced past import movements.

In the early 1970s, consumption increases led to sharp rises in petroleum imports. ${ }^{11}$ Net imports (crude and products) generally increased but remained less than 20 percent of consumption until 1969 (Figure 1). In 1970, crude oil production in the United States peaked at 9.6 million barrels per day (Figure 2). Consumption rose sharply, resulting in nearly a doubling of imports between 1970 and 1973 to 6.0 million barrels per day, 35 percent of consumption. The proportions of crude and product imports were roughly equal between 1965 and 1973 (Figure 3).

The price increases as a result of the 1973 oil embargo stopped the surging consumption but only for a couple of years. Consumption surpassed the 1973 level in 1976 and peaked at 18.8 million barrels per day in 1978 (Figure 2). Crude production continued to decline until 1977.The increased consumption and lower production gave rise to the
Figure 2. Petroleum Supply and Consumption, 1950-2015

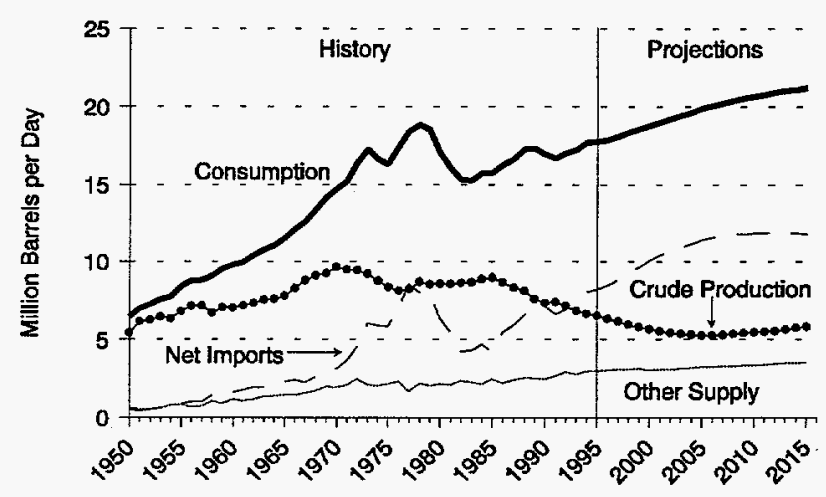

Source: History: 1950-1993: Energy Information Administration, Annual Energy Review 1994, DOE/EIA0384(94) (Washington, DC, July 1995). 1994: Energy Information Administration, Petroleum Supply Annual 1994, DOE/EIA-0340(94)/1 (Washington, DC, May 1995). Projections: AEO96 National Energy Modeling System run AEO96B.D101995C.

1977 peak in net imports of 8.6 million barrels per day, 46.5 percent of consumption. Crude net imports (as a percent of consumption) rose sharply in the mid-1970s, reaching 35.6 percent of consumption in 1977, and have dominated petroleum imports ever since. Product net imports, on the other hand, have followed a downward trend since $1973 .{ }^{12}$

The sharp price increases resulting from the Iran-Iraq war, combined with the end of petroleum price controls in the United States, brought down consumption. Consumption fell by 19 percent, or 3.7 million barrels per day, from 1978 to 1983 . Crude production, supported by the higher prices, increased slightly between 1978 and 1985 . As a result, net imports fell to 27.3 percent of consumption in 1985, a level that had not been seen since 1972.

In 1986, petroleum prices fell sharply as Saudi Arabia sought to increase market share, and domestic crude production again started to decline. Between 1985 and 1994, crude production fell 2.3 million barrels per day, 25.8 percent. Consumption began rising, with the exception of 1990 to 1991 , although it has yet to reach the 1978 peak. By 1994, net imports were approaching the 1977 levels. This time, however, declining production contributed a greater proportion of the increase than rising consumption.

\footnotetext{
${ }^{11}$ The lifting of import quotas allowed more imports into the country and forced U.S. producers to compete against cheaper foreign imports.

${ }^{12}$ In the 1970s, the decline in product imports reflected the incentives in the entitlements program to import crude oil and produce residual fuel and other products domestically. (Refer to The U.S. Petroleum Industry Past as Prologue 1970-1992, DOE/EIA-0572 (Washington, DC, September 1993), p. 47). In the 1980s, falling consumption led to surplus refining capacity which reduced the need for product imports.
} 
Figure 3. Crude Oil and Product Net Imports as a Percentage of Consumption, 1950-2015

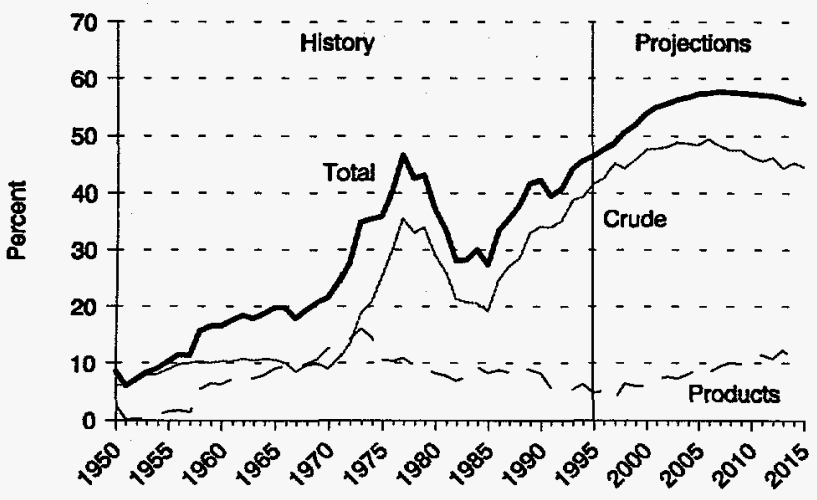

Source: Percentages calculated from the following data: History: Energy Information Administration, Annual Energy Review 1994, DOE/EIA-0384(94) (Washington, DC, July 1995). 1994: Energy Information Administration, Petroleum Supply Annual 1994, DOE/EIA-0340(94)/1 (Washington, DC, May 1995). Projections: AEO96 National Energy Modeling System run AEO96B.D101995C.

Helping to mitigate import dependence in recent years has been the increased contribution of "other" supply sources such as natural gas liquids and oxygenates. ${ }^{13}$ This category has ranged from 16 to 17 percent of consumption since 1991 , several percentage points higher than in the 1970s.

Petroleum consumption is projected to rise steadily from 1995 to 2015, according to the reference case projections in the AEO96. The rate of increase, however, is expected to slow in the later years of the forecast (a growth rate of 1.1 percent per year for 1995 to 2005 compared to 0.6 percent per year for 2005 to 2015) as a result of rising alternative fuel penetration and slowing economic and population estimates. Crude production is projected to continue to decline until 2005, although at a slower rate than the 1985 to 1995 period, but then make a gradual comeback between 2005 and 2015 as accumulating technological advances and rising prices stimulate faster recovery. ${ }^{14}$ Net imports, as a result, are expected to peak at 58 percent of consumption in 2007 and then decline slightly to 56 percent by 2015 . The contribution of "other" supplies is expected to increase over the forecast period but to remain nearly constant on a percentage basis.
Petroleum product net imports fell to 5.4 percent of consumption in 1993, the lowest level since 1958. Product net imports are projected to increase through the forecast, reaching 11 percent of consumption in 2015 . This reflects the more rapid growth in consumption relative to domestic refining capacity that is projected but remains below the 16 percent level of 1973 . While the proportion of demand represented by crude inputs to refineries is expected to be nearly the same in 2015 as in 1973, other supply sources such as natural gas liquids and oxygenates are projected at higher amounts. Crude oil will continue to dominate petroleum imports, rising to 49 percent of consumption in 2006 before dropping off to about 44 percent in 2015.

In the low world oil price case, ${ }^{15}$ the lower prices increase the consumption estimates but reduce the domestic crude production projections. Crude production declines throughout the forecast and does not show the upturn that is evident in the reference case. Net import dependence, as a result, peaks in 2015 at 68 percent of consumption (Figure 4). In the high world oil price case, crude production begins rising prior to 2000 and continues to increase through the forecast. The higher prices slow the consumption growth rate, and net imports as a percent of consumption remain close to current levels.

\section{Figure 4. Net Import Percentage of Consumption, Oil Price Cases, All Countries and Persian Gulf, 1995-2015}

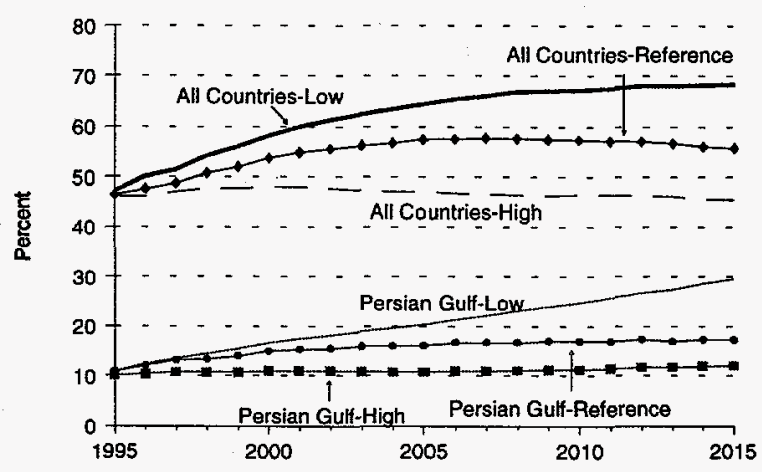

Source: AEO96 National Energy Modeling System runs AEO96B.D101995C, LWOP96.D101995B, HWOP96. D101995B. Persian Gulf estimates also use runs from the World Oil Refining, Logistics, and Demand (WORLD) Model.

\footnotetext{
${ }^{13}$ Also included in the "other" supply category are processing gains, stock changes, and unaccounted for crude oil.

${ }^{14}$ Energy Information Administration, Annual Energy Outlook 1996, DOE/EIA-0383(96) (Washington, DC, January 1996), p. 44.

${ }^{15}$ Energy Information Administration, Annual Energy Outlook 1996, DOE/EIA-0383(96) (Washington, DC, January 1996), pp. 6-8, $182-183$. The low world oil price projection shows a decline from current levels to $\$ 16.07$ per barrel (1994 dollars) in 2015. The high world oil price projection rises from $\$ 16.81$ per barrel in 1995 to $\$ 33.89$ per barrel in 2015 .
} 


\section{Diversity of Supply}

When imports rose sharply in the 1970s, the additional imports came primarily from the Persian Gulf, Nigeria, Libya, and Algeria. In the 1980s and early 1990s, imports from Canada and Mexico increased. ${ }^{16}$ In the AEO96 projections, imports from both Persian Gulf and North American sources are expected to increase. Imports from the Persian Gulf are expected to rise from about 10 percent of consumption in 1994 to about 17 percent in 2015 in EIA's reference case, exceeding the 1977 peak of 13.2 percent. In the low world oil price case, Persian Gulf net imports are projected to reach nearly 30 percent of consumption, an indication of that area's lower cost base and excess production capabilities. In the high world oil price case, however, the proportion of net imports from the Persian Gulf is expected to rise by only about 2 percentage points from 1994 levels, further demonstrating their role as "swing supplier" and remaining below the 1977 peak.

As noted earlier, imports from North American sources may be considered more secure than imports from distant sources such as the Persian Gulf. ${ }^{17}$ If net imports from Canada and Mexico are excluded from the dependence calculation, ${ }^{18}$ a somewhat different picture emerges (Figure 5). With this measure, dependence reached 43.2 percent in 1977 but was 33.9 percent in 1994. The United States does not reach the 1977 level until 2001 and peaks at about 47 percent in 2010 . This is about 10 percentage points lower than the dependence calculation using imports from all countries. Imports from other Caribbean Basin sources are also expected to increase.

\section{Summary}

Various methods for measuring dependence exist, and a determination of which sources of supply to include or exclude from the calculations must be made. The benefits of importing petroleum should be compared with the costs of remaining self-sufficient.
Figure 5. Net Import Percentage of Consumption, Reference Case, All Countries, Persian Gulf, and with Canada and Mexico Excluded, 1970-2015

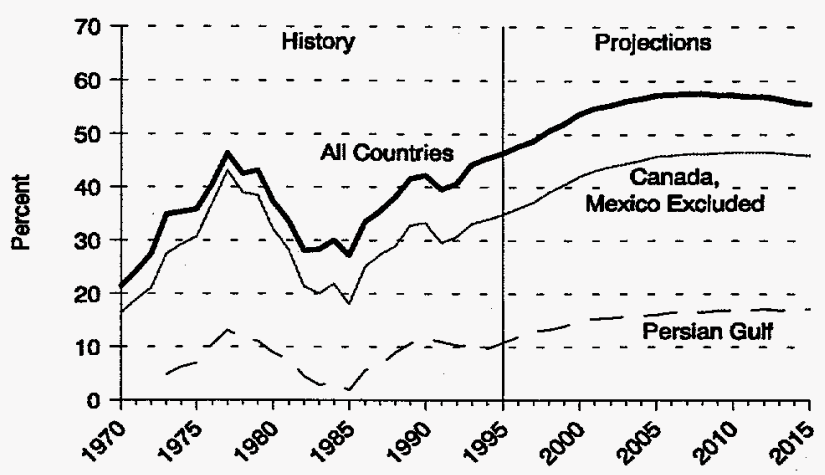

Source: History: 1970-1993: Energy Information Administration (EIA), Annual Energy Review 1994, DOE/EIA0384(94) (Washington, DC, July 1995), EIA, Monthly Energy Review, DOE/EIA-0035(96/05) (Washington, DC, May 1996). 1994: ElA, Petroleum Supply Annual 1994, DOE/EIA0340(94) $/$ (Washington, DC, May 1995). Projections: AE096 National Energy Modeling System run AEO96B.D101995C. Canada, Mexico, and Persian Gulf estimates also use runs from the World Oil Refining, Logistics, and Demand (WORLD) Model.

Import dependence measures show that the United States is becoming more dependent than ever on imported petroleum. As noted above, when measuring net imports from all countries, import dependence peaked in 1977 at 46.5 percent. The United States is close to that level currently, and import dependence is expected to continue to increase to about 58 percent before falling to 56 percent at the end of the forecast. Dependence on imports from the Persian Gulf area is projected to rise above historic levels. However, greater quantities of petroleum are also coming from sources closer to the United States, an important consideration in analyzing import dependence trends.

\footnotetext{
${ }^{16}$ Energy Information Administration, Monthly Energy Review, DOE/EIA-0035(96/05) (Washington, DC, May 1996), pp. 48-53.

${ }^{17}$ It is important to remember, however, that the petroleum trade is economically driven, so the North American supplies could go elsewhere if prices warranted.

${ }^{18}$ Canada and Mexico were chosen because of available historical and projected data. Similar calculations could be made excluding imports from other Caribbean and Latin American sources.
} 



\title{
Fuel Economy, Vehicle Choice, and Changing Demographics
}

\author{
(“Will Grandma Drive a 4x4?")
}

\author{
by Barry N. Cohen
}

\section{Introduction}

This paper describes the methodology used to forecast the truck share of light-duty vehicle (car and light truck) sales in the National Energy Modeling System (NEMS) transportation model. Given the marked difference in fuel economy standards for cars and light trucks, ${ }^{1}$ this share directly affects the forecasted levels of both oil consumption and carbon emissions. More generally, the presentation highlights the importance of considering structural shifts in developing long-term energy forecasts.

\section{Background}

Short-term-one- to two-year-energy forecasts assume that past trends in energy use and past relationships between economic and demographic factors and energy use will continue for the forecast period. In the short term, structural change is relatively unimportant as a cause of forecast error compared to the impact of unexpected changes in variables such as economic growth and energy prices. Those who work with long-term energy forecasts don't have this luxury. Over time capital stock turns over, new technology becomes available, consumer tastes change, and the composition of the work force and other demographic characteristics evolve. Thus, issues of structural change and technology market penetration are central considerations in all dimensions of long-term energy modeling.

This paper focuses on only one issue of importance in the transportation demand sector-the share of light trucks relative to total light-duty vehicle annual sales. It compares estimates of the light truck sales share based on extrapolating past trends to an approach that makes explicit assumptions concerning the impact of future demographic changes on vehicle choice decisions. A key development in the personal transportation market over the last two decades has been the steady increase in the sales share of light trucks (Figure 1).

Between 1970 and 1980, the truck sales share grew at an average annual rate of 3.0 percent. This rate increased to 5.2 percent between 1980 and 1990 but has recently slowed to a rate of 2.0 percent between 1992 and 1995. This phenomenon is important for understanding past gasoline consumption trends because the Corporate Average Fuel

Figure 1. Age and Sex Weighted Population Index of Truck Purchasers, 1970-1995

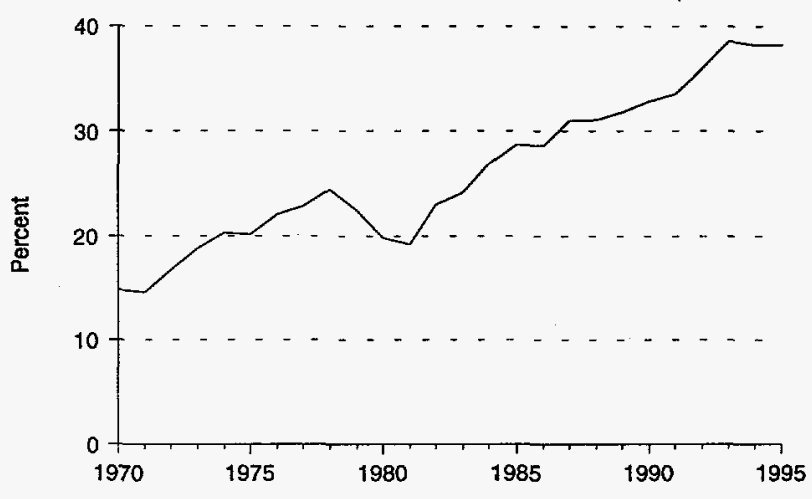

Source: Oak Ridge National Laboratory, Transportation Energy Data Book, Ed. 15, ORNL-6856, (Oak Ridge, TN, May 1995), pp. 3-17 through 3-29.

\footnotetext{
${ }^{1}$ The Corporate Average Fuel Economy requirement for automobiles is 27.5 miles per gallon (mpg); the standard for most light trucks, such as minivans, is $20.5 \mathrm{mpg}$, and the standard for $4 \times 4$ utility vehicles is $19.5 \mathrm{mpg}$.
} 
Economy (CAFE) standard for light trucks is 20.5 miles per gallon (mpg) compared to $27.5 \mathrm{mpg}$ for cars. If the light truck sales share in 1995 remained at the 1980 level of 20 percent rather than the 1995 level of 38 percent, 1995 gasoline consumption would have been significantly reduced. Average "on-the-road" fuel economy of light-duty vehicles (cars and light trucks) in this case would be 14 percent higher than the actual level achieved in 1995, and gasoline consumption would have been about 850 thousand barrels per day less. ${ }^{2}$

Developing the NEMS forecast through 2015 requires, among other things, specification of future trends in fleet composition with respect to cars and light trucks. Will fleet composition continue to evolve as we have seen over the past two decades? The Annual Energy Outlook 1996 $(A E O 96)^{3}$ forecast assumes this will not happen because the U.S. population is aging. Such aging is expected to constrict growth in market potential for light-duty trucks.

We know with a fair degree of certainty that, as the generation of "baby boomers" reaches age 55, the share of the population under the age of 55 will decrease by over 10 percentage points from the share maintained for several decades (Figure 2). We also know several important demographic characteristics of current light truck purchasers: more than three-fourths are male and over 85 percent are under 55 (Table 1 ). ${ }^{4}$ In developing the AEO96 light truck share forecast, the forecast provided by the NEMS macroeconomic model (based on the Data Resources, Inc, model) was adjusted downward to account for the aging of the population. By 2015, the initial truck share estimate of 48 percent was reduced to 43 percent. This reduced market share dampens the forecast level of gasoline consumption slightly within the time frame of the AEO96 forecast, which is 2015. Beyond 2015 , however, given the necessary time for the capital stock to turn over, the gasoline-savings effects of reducing the light truck share based on demographics are far more pronounced.

The methodology presumes the answer to the question raised in the subtitle of this paper. Older people are expected to be less likely buyers of light-duty trucks and recreational vehicles. Those becoming 55 in the forecast period are more likely to behave like consumers now in
Figure 2. Percentage of Population Under 55, 1970-2030

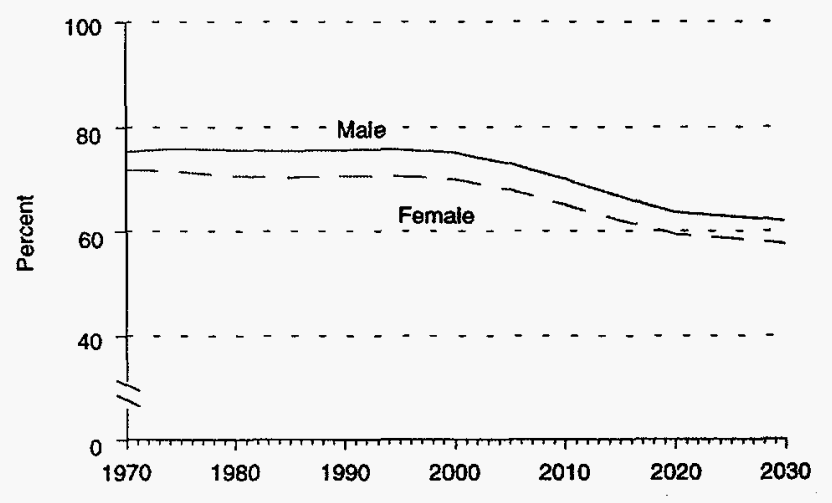

Source: U.S. Department of Commerce, Bureau of the Census, Projections of the Population of the United States, by Age, Sex, and Race: 1988 to 2080, Population Estimates and Projections, Series P-25, No. 1018, 1989.

the 55 and over group and thus will demand proportionately less in the way of recreational vehicles as they age. If this is wrong, the gasoline forecast may be on the low side in future years.

\section{Data Availability}

Information on the demographic characteristics of light truck buyers was obtained from the 1989 Buyers of New Compact Trucks, Summary Report, ${ }^{5}$ published by the Newsweek Corporation. In this report, two types of light trucks, pickup trucks and sport utility vehicles, are used as a proxy representing all light trucks sold domestically. This assumption was made because the Newsweek survey did not provide information on the characteristics of consumers who purchased other vehicles that are included in the CAFE definition of light trucks, e.g., minivans.

The first step was to aggregate the data across truck types in order to determine a combined age and sex distribution among truck purchasers.

\footnotetext{
${ }^{2}$ In 1995, the average on-the-road fuel economy of cars was 23.2 miles per gallon (mpg) while the average fuel economy of light trucks was $16.8 \mathrm{mpg}$. Overall light-duty vehicle fuel economy averaged just over $19 \mathrm{mpg}$. Had the sales share of light trucks not changed from the 1980 share of 20 percent, average light-duty vehicle fuel economy would have been $21.9 \mathrm{mpg}$ versus $19.2 \mathrm{mpg}$.

${ }^{3}$ Energy Information Administration, Annual Energy Outlook 1996, DOE/EIA-0383(96) (Washington, DC, January 1996).

${ }^{4}$ Permission to cite the 1989 Newsweek Survey of Truck Buyers was obtained from the Newsweek Rights \& Permissions Office represented by Jeanne Willcoxon in a letter dated October 7, 1995.

${ }^{5} 1989$ Buyers of New Compact Trucks: Summary Report, (Newsweek Co., New York, 1989).
} 
Table 1. Truck Buyer Characteristics, 1989 (Percent)

\begin{tabular}{ccc}
\hline Buyers & Pickup & Sport Utility \\
\hline Total $\ldots \ldots \ldots \ldots$ & 53 & 47 \\
Male $\ldots \ldots \ldots \ldots$ & 88 & 73 \\
Female $\ldots \ldots \ldots \ldots$ & 12 & 27 \\
& & \\
$<19 \ldots \ldots$ & 3 & 1 \\
$20-24 \ldots \ldots \ldots$ & 13 & 7 \\
$25-29 \ldots \ldots$ & 13 & 15 \\
$30-34 \ldots \ldots$ & 11 & 15 \\
$35-39 \ldots \ldots$ & 13 & 17 \\
$40-44 \ldots \ldots$ & 12 & 16 \\
$45-49 \ldots \ldots$ & 9 & 11 \\
$50-54 \ldots \ldots$ & 7 & $8 \ldots \ldots$ \\
$55-59 \ldots \ldots$ & 6 & 5 \\
$60-64 \ldots \ldots$ & 8 & 5 \\
$>65 \ldots \ldots$ & $\ldots \ldots \ldots$ & 3 \\
\hline
\end{tabular}

Source: 1989 Buyers of New Compact Trucks: Summary Report (Newsweek Co., New York, 1989).

Let: $\mathrm{P}_{\text {Type }}=$ the percentage of total light truck purchases of a given type.

$P_{\text {Sex }, \text { Type }}=$ the percentage of each type purchased by a given sex.

$P_{\text {Age,Type }}=$ the percentage of each type purchased by a given age group.

Then, aggregating across truck types:

$$
P_{\text {Sex }, \text { Age }}=\sum_{\text {Type }} P_{\text {Type }} \cdot P_{\text {Sex }, \text { Type }} \cdot P_{\text {Age,Type }}
$$

as displayed below. A summation across sex within each age group provides the age distribution of light truck purchasers, depicted in Table 2 and Figure 3.

Looking at the cumulative percentages (Figure 4), approximately 85 percent of all light truck purchases are made by people under 55 years old. Of this group, approximately 81 percent are men and 19 percent are women (Table 2). The historical and projected proportion of the population under 55 years is displayed in Figure 2.

\section{Methodology}

The annual truck share of light-duty vehicle sales in the AEO96 forecast was calculated by multiplying the NEMS, DRI-based, forecast of light truck sales by a weighted population index that increasingly falls below 1.0 in the later years of the forecast as the proportion of the population under age 55 declines (Figure 5).

\section{Estimate of Truck Sales Share Based on an Extrapolation of Recent Trends}

The unadjusted share of total light-duty vehicle purchases accounted for by trucks is extrapolated using the 1982 and 1992 values as anchor points and an assumed maximum value. The functional form of the curve is as follows:

$$
L T S_{T}=L T S_{1982}+\left(L T S_{\text {Max }}-L T S_{1982}\right) \cdot\left(1-\exp ^{-k(T-1982)}\right)
$$

where LTS indicates the light truck share of total lightduty vehicle sales, and the constant, $k$, is determined as follows:

$$
k=\frac{-L n\left[1-\frac{\left(L T S_{1992}-L T S_{1982}\right)}{\left(L T S_{M a x}-L T S_{1982}\right)}\right]}{10}
$$

Using the data below, $\mathrm{k}$ is calculated and the curve is explicitly defined.

$$
\text { Extrapolation Inputs }
$$

$\begin{array}{cc}\text { LTS }_{1982} & 0.230 \\ \text { LTS }_{\text {1992 }} & 0.353 \\ \text { LTS }_{\text {Max }} & 0.550 \\ \text { k } & 0.049\end{array}$

The extrapolated estimates of the light truck share through 2015 are close to other recent estimates that project that trucks will account for nearly half of all lightduty vehicle sales by $2015 .^{6}$ The specific methodology used in AEO96 to develop a demographic index to dampen future truck sales is detailed below. This index was used to dampen all light truck sales. It is certainly not the only index that could have been developed.

${ }^{6}$ The DRI/McGraw-Hill estimates of the annual light truck sales share were similar to the trend-based estimate developed here. By 2015, the DRI estimate of the light truck sales share reached 48 percent. 
Table 2. Overall Light Duty Truck Purchases, 1989 (Percent)

\begin{tabular}{|c|c|c|c|c|}
\hline Age Distribution & Male & Female & Total & Cumulative \\
\hline$<19$ & 2 & 0 & 2 & 2 \\
\hline$\ldots \ldots \ldots \ldots \ldots$ & 8 & 2 & 10 & 12 \\
\hline $25-29 \ldots \ldots \ldots \ldots \ldots \ldots \ldots \ldots \ldots$ & 11 & 3 & 14 & 26 \\
\hline 30-34 $\ldots \ldots \ldots \ldots \ldots \ldots \ldots \ldots \ldots \ldots$ & 10 & 3 & 13 & 39 \\
\hline$\ldots \ldots \ldots, \ldots, \cdots$, & 12 & 3 & 15 & 54 \\
\hline $40-44 \ldots .$. & 11 & 3 & 14 & 68 \\
\hline$\ldots \ldots \ldots \ldots \ldots \ldots \ldots$ & 8 & 2 & 10 & 78 \\
\hline$\ldots \ldots \ldots \ldots \ldots \ldots \ldots$ & 6 & 1 & 7 & 85 \\
\hline$\ldots \ldots \ldots \ldots \ldots$ & 5 & 1 & 6 & 91 \\
\hline $60-64 \ldots \ldots \ldots \ldots \ldots \ldots \ldots \ldots \ldots \ldots$ & 3 & 1 & 4 & 95 \\
\hline$>65 \ldots \ldots \ldots \ldots \ldots \ldots \ldots \ldots \ldots \ldots \ldots \ldots \ldots \ldots \ldots$ & 4 & 1 & 5 & 100 \\
\hline Total $\ldots \ldots \ldots \ldots \ldots \ldots \ldots \ldots \ldots, \ldots$ & 81 & 19 & 100 & \\
\hline
\end{tabular}

Source: 1989 Buyers of New Compact Trucks: Summary Report (Newsweek Co., New York, 1989).

Figure 3. Age Distribution of Truck Purchasers, 1989

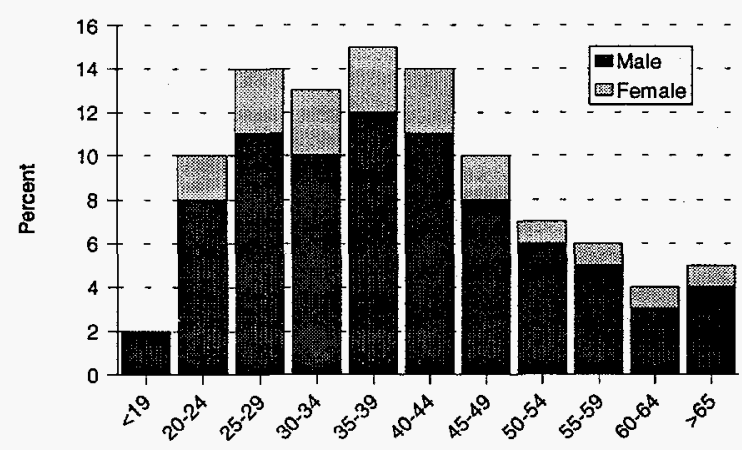

Source: 1989 Buyers of New Compact Trucks: Summary Report (Newsweek Co., New York: 1989).

However, given the aging of the population and assuning that future grandmas do not increase their propensity to purchase light trucks, any approach to adjust truck sales for an aging population would dampen light truck sales beyond 2000.

\section{Estimate of Truck Sales Adjusted by a Weighted Population Index}

Since people under the age of 55 are responsible for 85 percent of all light truck purchases (Table 2), declines in this share of the population have a moderating influence relative to an estimate of light truck sales based on
Figure 4. Cumulative Percentage of Truck Purchases by Age Category, 1989

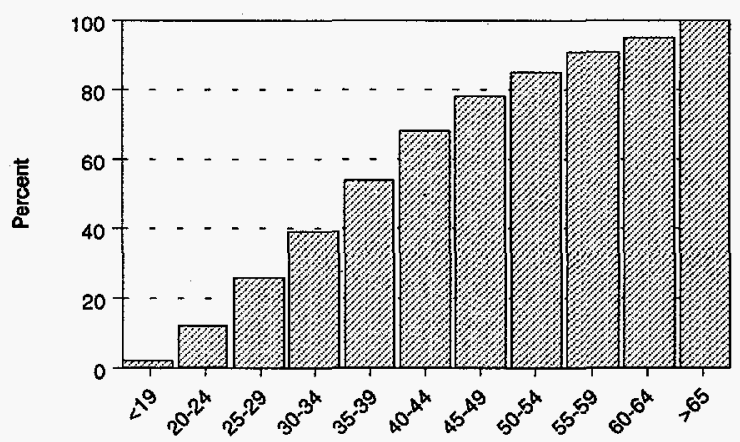

Source: 1989 Buyers of New Compact Trucks: Summary Report (Newsweek Co., New York, 1989).

extrapolating recent history. The population index is weighted by the male/female distribution (Table 2) of truck buyers. Thus it considers both the dampening effect on truck sales of the general aging of the population and the fact that women, who have historically not purchased a high percentage of light trucks, become a larger fraction of the population based on current longevity statistics. Specifically:

Let: $P_{\text {Sex, Age }} \quad=$ the fraction of all light-duty truck buyers of a given sex and age group

$$
\Pi_{\mathrm{Sex}, \mathrm{Age}<55, \mathrm{~T}}=\text { the percentage of the population }
$$
under age 55 , by sex, in year $\mathrm{T}$ 
Figure 5. Age and Sex Weighted Population Index of Truck Purchasers, 1970-2030

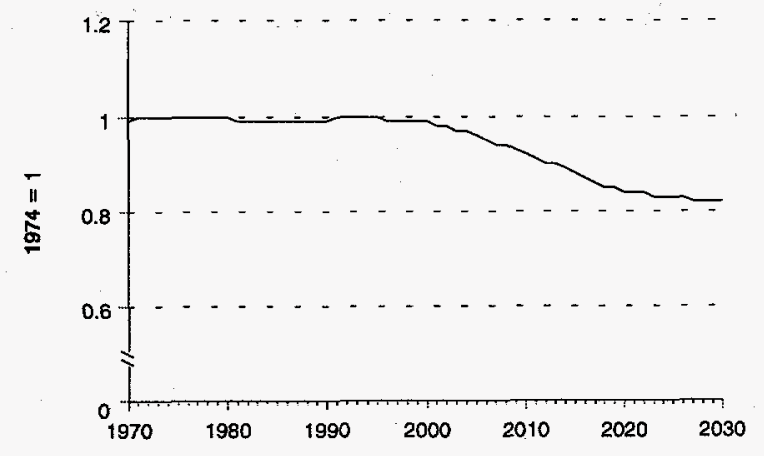

Source: Energy Information Administration, Office of Integrated Analysis and Forecasting.

The weighted share of the population under the age of 55 is then:

$$
P<55_{T}=\sum_{\text {Sex }}\left[\Pi_{\text {Sex }, \text { Age }<55, T} \cdot\left(\sum_{\text {Age }} P_{\text {Sex }, \text { Age }}\right)\right]
$$

This share is subsequently indexed $(1990=1.0)$. The effect of the aging of the "baby boomers" is dramatic (Figure 5). The truck-buying segment of the population, assuming the current age and sex characterization of truck purchasers remains constant, declines by nearly 15 percentage points by 2015 .

\section{Truck Sales Forecast}

For any forecast year, the population-adjusted estimate of the light truck share is calculated simply by multiplying the extrapolated estimates by the demographic index described above and displayed in Figure 5. Again, the results are striking. The truck sales share levels off at about 43 percent in 2015 and remains roughly constant through 2030 .

\section{Conclusions}

Forecasts of total light-duty vehicle sales in NEMS are provided by the DRI-based macroeconomic model. The estimated sales share of light trucks considers both recent trends in truck sales and expected significant changes in the demographic makeup of future light-duty vehicle purchasers. The population-adjusted estimates of the truck sales share in 2005, 2010, and 2015 are below the trend-based sales share by 2,4 , and 6 percentage points, respectively. ${ }^{7}$ By 2015 , this reduction in the truck sales share results in a 0.6 -percent increase in average "on-theroad" personal vehicle fuel economy. This translates directly into a gasoline savings of over 450 thousand barrels per day in 2015. Beyond 2015, as older vehicles are replaced, the steady 43-percent estimated truck sales share would have a greater dampening effect on gasoline consumption relative to the trend-based extrapolation which goes above 50 percent by 2020 (Figure 6). In the long term, as the vehicle stock is replaced, each percentage decrease in the truck sales share dampens fuel consumption more significantly because, on average, light truck fuel economy is about 25 percent less than car fuel economy. This conclusion, of course, also assumes that current fuel-economy related legislation remains unchanged over the forecast horizon.

Figure 6. Truck Sales Share of Light-Duty Vehicle Purchases, 1970-2030

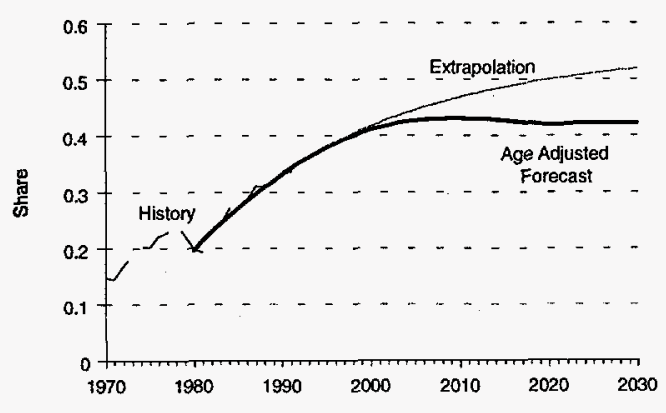

Source: Energy Information Administration, Office of Integrated Analysis and Forecasting.

\footnotetext{
7The DRI/McGraw-Hill estimates of the annual light truck sales share were similar to the trend-based estimate developed here. By 2015, the DRI estimate of the light truck sales share reached 48 percent.
} 



\title{
Annual Energy Outlook Forecast Evaluation
}

\author{
by Scott B. Sitzer
}

\section{Introduction}

This paper presents an analysis of the forecast record of the Annual Energy Outlook ( $A E O) .{ }^{1}$ It compares the projections for major energy variables from the reference case for each of the AEOs published from April 1983 through January 1993 with actual data for selected years. ${ }^{2}$ The purpose of the analysis is to provide one performance measure of the forecasts, namely their accuracy. However, while important, forecasting is not the only reason for maintaining the models that the Energy Information Administration (EIA) uses in producing the $A E O$.

The primary reason for modeling in EIA is to provide a tool for policy analysis. While the reference case forecasts used in this evaluation represent a baseline for policy analysis, many readers use the reference case projections as a "most likely," or highest-probability forecast. In fact, a better characterization might be "business as usual," because a key assumption of the reference case forecasts is that current laws and regulations will remain in effect throughout the forecast horizon. This assumption, while necessary to provide a baseline against which changes in policy can be tested, also virtually guarantees that the forecasts will be in error, as laws and regulations pertinent to energy markets have changed considerably over the years. Just in the period analyzed in this paper, legislation and policies including the National Appliance and Energy Conservation Act of 1987, the Natural Gas Wellhead Decontrol Act of 1989, the Clean Air Act Amendments of 1990 (CAAA90), the Energy Policy Act of 1992, the repeal of the Fuel Use Act, various Federal Energy Regulatory Commission (FERC) orders, and the North American Free Trade Agreement, among others, have become law or have been adopted as explicit government policies. All of these have had significant impact on energy supply, demand, and prices. But because of the business-as-usual assumption, the impacts were not incorporated in the projections made before their enactment or effective dates.

In several cases, EIA's models were used to evaluate some of the potential impacts of these changes in laws and regulations before they were enacted, thus fulfilling EIA's designated role in policy analysis. For example, EIA provided comprehensive analysis to the House Energy and Commerce Committee concerning the impacts of the CAAA90 on the coal and electricity industries. In other cases, the models have been used to analyze policies which were eventually rejected; a prime example is the British thermal unit (Btu) tax proposed in early 1993. Both of these uses of the models illustrate the importance of maintaining a modeling capability apart from the forecasting function and also show how assuming current laws and regulations facilitates the analytical process.

A second important assumption embedded in the $A E O$ forecasts is that normal weather patterns will prevail. In fact, "normal" weather is a rare occurrence for an entire calendar year. While the AEO forecasting models have not generally been used for analysis of weather conditions on energy markets, ${ }^{3}$ temperatures that are colder or warmer than normal for sustained periods have had, and will continue to have, an obvious impact on energy consumption. The assumption of normal weather is therefore similar to assuming no change in current laws and regulations, in that we know that the weather will rarely, if ever, be normal in any given year.

Nevertheless, accuracy will continue to be an important measure of the models' performance, both for users and for those responsible for the projections. For that reason, an analysis of the accuracy of the $A E O$ forecasts is important.

Because the format of the $A E O$ has changed significantly over the years, the selection of publications, variables, and

\footnotetext{
${ }^{1}$ Energy Information Administration, Annual Energy Outlook 1996, DOE/EIA-0383(96) (Washington, DC, January 1996) for the most recent AEO.

${ }^{2}$ For an analysis of ElA's record for forecasts made from 1977 through 1993, see Cohen, B.; Peabody, G.; Rodekohr, M.; and Shaw, S., "A History of Mid-Term Energy Projections: A Review of the Annual Energy Outlook Projections," February 1995, unpublished manuscript.

${ }^{3}$ The Short-Term Integrated Forecasting System, which produces quarterly forecasts for up to two years in the future, is EIA's primary tool for near-term analysis of the impact of weather changes on energy markets.
} 
the years to which the forecasts are to be compared must inevitably be a judgment call. Not every year was projected for each AEO; the Annual Energy Outlook $1982^{4}$ (AEO82), for example, only forecasted energy markets through 1990. For the purpose of this evaluation, each AEO from 1982 through 1993 was used for the years 1985 through 1990 and 1995, and only a subset of the most important energy variables was included. However, the information provided herein gives a relatively comprehensive view of the performance of the $A E O$ for the most important energy variables.

The next section of this paper provides an overview of the findings. Following the overview, each of the major variables is discussed in turn. Some concluding remarks and caveats complete the analysis.

\section{Overview}

Table 1 provides a summary of the average absolute forecast errors, ${ }^{5}$ expressed as percentage deviations from actual, for each of the major variables included in the evaluation analysis. ${ }^{6}$ As the table indicates, the forecast performance of the consumption, supply, and macroeconomic variables has generally been very good; the performance of net imports has been less accurate; and the performance of prices, with the exception of residential electricity prices, has been poor.

Each of the consumption, supply, and macroeconomic variables, with the exception of the net import variables, has been projected with an average absolute error of 6 percent or less. For both total energy consumption and total electricity sales, the most accurately projected variables during this period, the average absolute percent error was under 2 percent. Net import errors have ranged from about 12 percent for petroleum to 20 percent for natural gas. For prices, forecasting has proven to be much more of a challenge. Average errors for the world oil price, the price of coal to electric utilities, and the price of natural gas at the wellhead ranged from 47 to 120 percent over the period, with natural gas at the wellhead proving to have the highest average forecast error of the major energy variables. Residential electricity price projections,
Table 1. Average Absolute Percent Errors for AEO Forecasts, 1982-1993

\begin{tabular}{|c|c|}
\hline Variable & $\begin{array}{c}\text { Average } \\
\text { Absolute } \\
\text { Percent Error }\end{array}$ \\
\hline \multicolumn{2}{|l|}{ Consumption } \\
\hline Total Energy Consumption ... . & 1.8 \\
\hline Total Petroleum Consumption .. & 3.2 \\
\hline Total Natural Gas Consumption . . . . . . & 6.0 \\
\hline Total Coal Consumption & 2.9 \\
\hline Total Electricity Sales . . . . . . . . . . & 1.8 \\
\hline \multicolumn{2}{|l|}{ Supply } \\
\hline Crude Oil Production & 5.1 \\
\hline Natural Gas Production . & 5.4 \\
\hline Coal Production . & 3.8 \\
\hline \multicolumn{2}{|l|}{ Imports and Exports } \\
\hline Net Petroleum Imports & 12.0 \\
\hline Net Natural Gas Imports & 20.0 \\
\hline Net Coal Exports . . . . . . . . . . . & 17.1 \\
\hline \multicolumn{2}{|l|}{ Price and Macroeconomic Variables } \\
\hline World Oil Prices . . . . . . . . . . . . & 82.2 \\
\hline Natural Gas Wellhead Prices . . . . . . . & 120.4 \\
\hline Coal Prices to Electric Utilities & 47.1 \\
\hline Residential Electricity Prices . . . . . . . & 11.0 \\
\hline Disposable Personal Income . . . . . . . & 5.4 \\
\hline
\end{tabular}

$\mathrm{AEO}=$ Annual Energy Outlook.

Source: Calculated from Tables 2 through 17.

however, fared much better, with an 11-percent average error.

While the following sections discuss the underlying results in some detail, it is clear from the table that quantities are more amenable to the forecasting methods used in the $A E O$ than prices; that the errors in forecasting prices have not, in general, affected the accuracy of projected quantities; and that natural gas has tended to have the highest average forecast error within each of the major

\footnotetext{
${ }^{4}$ Energy Information Administration, Annual Energy Outlook 1982, DOE/EIA-0383(82) (Washington, DC, April 1983).

${ }^{5}$ The average absolute errors displayed in Table 1 are the average absolute percentage errors for each variable shown in Tables 2 through 17. This measure is computed as the mean of all of the absolute percentage errors shown for each $A E O$, for each year projected, for a given variable.

${ }^{6}$ It should be noted that the evaluations given in this paper are for the $A E O$ reference cases only. In fact, each $A E O$ has provided a range of projections, based on varying assumptions concerning world oil prices and future economic activity. In many cases, the range of forecasts has, in fact, encompassed the eventual outcome of the variables evaluated. Nevertheless, in order to keep the analysis to manageable levels, the point forecasts represented by the reference case have been used in this paper. An obvious extension would be an analysis of the forecast ranges and the impact of differing assumptions on forecast accuracy.
} 
categories of consumption, supply, imports, and prices. Some of the major factors leading to inaccurate forecasts include the assumption in the earlier AEOs that world oil prices would continue to be determined largely by the Organization of Petroleum Exporting Countries (OPEC) oil cartel; the underestimates of the impacts of technology improvements in fossil fuel production; the impacts of changes in laws and regulations on natural gas prices; the treatment of fuel supply contract provisions for natural gas and coal as fixed and binding; and deviations from normal weather, in contrast to the major assumptions made by EIA as discussed above.

\section{Energy Consumption}

\section{Total Energy Consumption}

Total energy consumption forecasts (excluding nonelectric renewables, which have not been forecast across all AEOs) have shown a generally good track record for most of the $A E O$ publications. The overall average absolute percent error for the period examined here is 1.8 percent (Table 2), with the largest errors occurring in forecasts for the year 1986 (2.4 percent), and the smallest errors in forecasts for 1987 (1.1 percent).

In terms of the $A E O$ publications, the Annual Energy Outlook $1986^{7}$ (AEO86) had the largest absolute error at 3.0 percent. There was a significant underestimate of energy consumption for most of the projected years in the AEO86, in part due to the high fossil fuel prices assumed for the publication, which was completed prior to the 1986 collapse in oil prices and published early in 1987. Subsequent to the $A E O 86$, there was general improvement in the forecast record, as EIA's experience with lowerpriced energy markets expanded. It is worth noting, however, that the overall average errors for oil price forecasts in the $A E O 86$ were actually better than in the preceding $A E O$ s and also better than in some subsequent ones, since some of the subsequent $A E O$ s projected world oil prices that were too low for the years 1989 and 1990 in particular.

One of the aspects of demand modeling that is important in the evaluation of the forecasts is the effect of regulations such as appliance and automobile efficiency standards. By incorporating such standards, some decisions that would otherwise be made by the interaction of supply and demand factors are in fact set by fiat, helping to reduce some of the uncertainty associated with the forecasts and reducing at least one source of forecast error.

\section{Total Petroleum Consumption}

Total petroleum consumption forecasts have averaged a 3.2-percent error during this period (Table 3 ). The least accurate forecast year was 1988 , for which the AEOs averaged about 0.75 million barrels per day lower than the actual consumption of 17.3 million barrels per day. In 1988 , the forecasts of the world oil price were also consistently too high, with an average error of 93 percent, the highest for any year other than 1995. In addition, the 1988 forecasts of disposable personal income tended to be too low in most of the $A E O$ publications, which would also lead to an underestimate of demand.

$A E O 82$, the earliest publication considered in this analysis, ${ }^{8}$ had the highest average error for petroleum consumption at 5.3 percent. The projections for the years 1985 through 1987 were above actual demand; the errors for 1988 through 1990 were much smaller and in the opposite direction. The AEO82 forecast for the year 1985 had the highest error of all of the petroleum forecasts evaluated. Residential and commercial consumption was projected to be more than 400 thousand barrels per day higher in 1985 than actual, and consumption of petroleum for electricity generation was projected to be more than 1.3 million barrels per day higher in 1985, or more than triple the actual. Both these numbers were reduced in the Annual Energy Outlook $1983^{\circ}$ (AEO83) and are more accurate. Although the AEO82 projection for 1990 is precisely correct at $\mathbf{1 6 . 9 9}$ million barrels per day, the sectoral projections were less accurate. Residential and commercial demand was projected to be about 600 thousand barrels per day higher, transportation 2.5 million barrels per day lower, and consumption for electricity generation 1.2 million barrels per day higher than actual. Residential and commercial demand was better characterized by the following year. Also, between $A E O 82$ and $A E O 83$, the role of natural gas in the electricity sector had been reevaluated. The projection of natural gas consumption by the electricity sector was up sharply in AEO83 with oil greatly reduced.

Following AEO82, residential and commercial consumption for oil remained rather close to actual, although missing the slight downturn in 1990. A general characterization of energy demand is that there was a tendency to underpredict in the publications after the

\footnotetext{
${ }^{7}$ Energy Information Administration, Annual Energy Outlook 1986, DOE/EIA-0383(86) (Washington, DC, February 1987).

${ }^{8}$ Energy Information Administration published earlier forecasts in its Annual Report to Congress; these have not been included in this report.

${ }^{9}$ Energy Information Administration, Annual Energy Outlook 1983, DOE/EIA-0383(83) (Washington, DC, May 1984).
} 
Table 2. Total Energy Consumption, Actual vs. Forecasts

\begin{tabular}{|c|c|c|c|c|c|c|c|c|}
\hline Publications & 1985 & 1986 & 1987 & 1988 & 1989 & 1990 & 1995 & $\begin{array}{c}\text { Average } \\
\text { Absolute } \\
\text { Error }\end{array}$ \\
\hline \multicolumn{9}{|c|}{ (quadrillion Btu) } \\
\hline AEO82 & 76.3 & 76.9 & 77.2 & 78.0 & 78.9 & 80.4 & & 1.8 \\
\hline AEO $83 \ldots \ldots \ldots \ldots$ & 75.2 & 76.8 & 78.3 & 79.6 & 80.7 & 81.7 & 86.1 & 1.1 \\
\hline AEO $84 \ldots \ldots \ldots \ldots$ & 75.7 & 76.7 & 78.5 & 80.3 & 81.9 & 83.5 & 90.1 & 1.6 \\
\hline AEO85 $\ldots \ldots \ldots \ldots$ & 74.8 & 75.8 & 77.1 & 78.4 & 79.5 & 80.4 & 84.3 & 1.4 \\
\hline AEO $86 \ldots \ldots \ldots \ldots$ & & 74.3 & 76.1 & 77.0 & 77.5 & 78.6 & 83.1 & 2.4 \\
\hline AE087 & & & 76.2 & 77.2 & 78.8 & 79.9 & 85.0 & 2.0 \\
\hline AEO89 $\ldots \ldots \ldots \ldots \ldots$ & & & & 79.4 & 80.6 & 81.6 & 85.8 & 0.8 \\
\hline AEO90 $\ldots \ldots \ldots \ldots$ & & & & & 80.8 & 82.0 & 88.0 & 0.7 \\
\hline AEO91 $\ldots$ & & & & & & 81.5 & 85.7 & 0.9 \\
\hline AEO92 & & & & & & & 87.3 & 0.0 \\
\hline AEO93 $\ldots \ldots \ldots$ & & & & & & & 88.4 & 1.2 \\
\hline Actual & 74.0 & 74.3 & 76.9 & 80.2 & 81.3 & 81.3 & 87.2 & \\
\hline \multicolumn{9}{|l|}{ Average Absolute } \\
\hline Error..$\ldots \ldots \ldots \ldots$ & 1.5 & 1.8 & 0.8 & 1.7 & 1.6 & 1.1 & 1.8 & 1.5 \\
\hline \multicolumn{9}{|c|}{ (percent error) } \\
\hline AEO82 & 3.1 & 3.5 & 0.4 & -2.8 & -3.0 & -1.1 & & 2.3 \\
\hline AEO83 & 1.6 & 3.4 & 1.8 & -0.8 & -0.8 & 0.5 & -1.3 & 1.5 \\
\hline AEO84 & 2.3 & 3.2 & 2.1 & 0.1 & 0.7 & 2.8 & 3.3 & 2.1 \\
\hline AEO85 $\ldots \ldots \ldots \ldots$ & 1.1 & 2.0 & 0.3 & -2.3 & -2.2 & -1.1 & -3.3 & 1.8 \\
\hline AEO86 $\ldots$ & & 0.0 & -1.0 & -4.0 & -4.7 & -3.3 & -4.7 & 3.0 \\
\hline AEO $87 \ldots \ldots$ & & & -0.9 & -3.8 & -3.1 & -1.7 & -2.5 & 2.4 \\
\hline AEO89 $\ldots \ldots \ldots$ & & & & -1.0 & -0.9 & 0.4 & -1.6 & 1.0 \\
\hline AEO90 $\ldots \ldots \ldots \ldots$ & & & & & -0.6 & 0.9 & 0.9 & 0.8 \\
\hline AEO91 $\ldots \ldots \ldots \ldots$ & & & & & & 0.3 & -1.7 & 1.0 \\
\hline AEO92 $\ldots \ldots \ldots \ldots$ & & & & & & & 0.1 & 0.1 \\
\hline AEO93 $\ldots \ldots \ldots \ldots$ & & & & & & & 1.4 & 1.4 \\
\hline \multicolumn{9}{|l|}{ Average Absolute } \\
\hline Percent Error ... & 2.1 & 2.4 & 1.1 & 2.1 & 2.0 & 1.3 & 2.1 & 1.8 \\
\hline
\end{tabular}

AEO = Annual Energy Outlook

Btu $=$ British thermal unit.

Notes: Excludes nonelectric renewables. Data for 1995 are preliminary.

Sources: History: Energy Information Administration (EIA), Monthly Energy Review, DOE/EIA-0035 (96/03) (Washington, DC, March 1996). Projections: EIA, various issues, Annual Energy Outlook, DOE/EIA-0383(82-93) (Washington, DC, April 1983 through January 1993).

Annual Energy Outlook 1984 ${ }^{10}$ (AEO84). There was an assumption in the consumption models that customers would purchase technologies that minimized the life-cycle cost, an assumption which resulted in overly optimistic efficiency improvements. The Annual Energy Outlook $1985^{11}$ (AEO85) shows this impact in the residential and commercial sectors. In the early forecasts, industrial consumption of oil was overestimated, partially reflecting somewhat optimistic assumptions about the growth of energy-intensive industries but also due to an underestimation of the potential growth of natural gas in an era of high gas prices. Later projections were somewhat underestimated due to assumptions of higher efficiency gains.

\footnotetext{
${ }^{10}$ Energy Information Administration, Annual Energy Outlook 1984, DOE/EIA-0383(84) (Washington, DC, January 1985).

${ }^{11}$ Energy Information Administration, Annual Energy Outlook 1985, DOE/EIA-0383(85) (Washington, DC, February 1986).
} 
Table 3. Total Petroleum Consumption, Actual vs. Forecasts

\begin{tabular}{|c|c|c|c|c|c|c|c|c|}
\hline Publications & 1985 & 1986 & 1987 & 1988 & 1989 & 1990 & 1995 & $\begin{array}{c}\text { Average } \\
\text { Absolute } \\
\text { Error }\end{array}$ \\
\hline \multicolumn{9}{|c|}{ (million barrels per day) } \\
\hline AEO82 & 18.00 & 17.89 & 17.55 & 17.24 & 16.98 & 16.99 & & 0.86 \\
\hline AEO83 & 15.82 & 16.13 & 16.37 & 16.50 & 16.56 & 16.63 & 17.37 & 0.40 \\
\hline AEO84 & 15.77 & 15.76 & 16.01 & 16.27 & 16.48 & 16.74 & 18.00 & 0.52 \\
\hline AEO85 & 15.72 & 15.74 & 15.97 & 16.01 & 16.06 & 16.08 & 16.53 & 0.84 \\
\hline AEO86 & & 16.07 & 16.29 & 16.05 & 16.07 & 16.15 & 16.46 & 0.86 \\
\hline AEO 87 & & & 16.52 & 16.66 & 16.96 & 17.06 & 17.72 & 0.24 \\
\hline AEO89 & & & & 17.01 & 17.20 & 17.44 & 17.82 & 0.24 \\
\hline AEO90 & & & & & 17.24 & 17.41 & 18.21 & 0.34 \\
\hline AEO91 ... & & & & & & 16.95 & 17.34 & 0.20 \\
\hline AEO9 $2 \ldots \ldots \ldots$ & & & & & & & 17.80 & 0.10 \\
\hline AEO93 $\ldots \ldots \ldots$ & & & & & & & 18.15 & 0.45 \\
\hline Actual & 15.73 & 16.28 & 16.67 & 17.28 & 17.33 & 16.99 & 17.70 & \\
\hline \multicolumn{9}{|l|}{ Average Absolute } \\
\hline Error .......... & 0.60 & 0.61 & 0.51 & 0.75 & 0.63 & 0.37 & 0.46 & 0.55 \\
\hline \multicolumn{9}{|c|}{ (percent error) } \\
\hline AEO82 & 14.5 & 9.9 & 5.3 & -0.2 & -2.0 & 0.0 & & 5.3 \\
\hline AEO83 & 0.6 & -0.9 & -1.8 & -4.5 & -4.4 & -2.1 & -1.9 & 2.3 \\
\hline AEO84 & 0.3 & -3.2 & -3.9 & -5.9 & -4.9 & -1.5 & 1.7 & 3.0 \\
\hline AEO85 $\ldots$ & 0.0 & -3.3 & -4.2 & -7.4 & -7.3 & -5.3 & -6.6 & 4.9 \\
\hline AEO8 $6 \ldots \ldots \ldots$ & & -1.3 & -2.3 & -7.1 & -7.2 & -4.9 & -7.0 & 5.0 \\
\hline AEO87 $\ldots \ldots \ldots$ & & & -0.9 & -3.6 & -2.1 & 0.4 & 0.1 & 1.4 \\
\hline AEO89 & & & & -1.6 & -0.7 & 2.7 & 0.7 & 1.4 \\
\hline AEO90 & & & & & -0.5 & 2.5 & 2.9 & 1.9 \\
\hline AEO91 $\ldots \ldots \ldots$ & & & & & & -0.2 & -2.1 & 1.1 \\
\hline AEO92 & & & & & & & 0.5 & 0.5 \\
\hline AEO93 & & & & & & & 2.5 & 2.5 \\
\hline \multicolumn{9}{|l|}{ Average Absolute } \\
\hline Percent Error & 3.8 & 3.7 & 3.1 & 4.3 & 3.6 & 2.2 & 2.6 & 3.2 \\
\hline
\end{tabular}

$\mathrm{AEO}=$ Annual Energy Outlook

Notes: Data for 1995 are preliminary.

Sources: History: Energy Information Administration (EIA), Monthly Energy Review, DOE/EIA-0035 (96/03) (Washington, DC, March 1996). Projections: EIA, various issues, Annual Energy Outlook, DOE/EIA-0383(82-93) (Washington, DC, April 1983 through January 1993).

Through most of the forecasts, transportation consumption was significantly underpredicted. World oil prices were projected to be too high, and the reaction to the higher prices in terms of vehicle efficiency improvements, estimated too high, and vehicle miles traveled, estimated too low, led to estimates that were on the order of 1 million barrels per day too low. This improved significantly in the Annual Energy Outlook $1987^{12}$ (AEO87), which contained the first set of projections after the oil price collapse in 1986.

\section{Total Natural Gas Consumption}

The overall error for natural gas consumption forecasts for the period studied was 6.0 percent, but this is somewhat skewed by the 11.1-percent error for 1995 (Table 4). Prior to 1995 , the year with the highest forecast error, at 6.9 percent, proved to be 1986. Except for AEO82, all of the errors for 1986 were overpredictions, despite price projections for 1986 that tended to be on the high side. For 1995, all of the AEOs through the Annual Energy Outlook

\footnotetext{
${ }^{12}$ Energy Information Administration, Annual Energy Outlook 1987, DOE/EIA-0383(87) (Washington, DC, March 1988).
} 
Table 4. Total Natural Gas Consumption, Actual vs. Forecasts

\begin{tabular}{|c|c|c|c|c|c|c|c|c|}
\hline Publications & 1985 & 1986 & 1987 & 1988 & 1989 & 1990 & 1995 & $\begin{array}{c}\text { Average } \\
\text { Absolute } \\
\text { Error }\end{array}$ \\
\hline \multicolumn{9}{|c|}{ (trillion cubic feet) } \\
\hline AEO82 & 15.90 & 15.72 & 15.71 & 16.10 & 16.57 & 17.06 & & 1.53 \\
\hline AEO83 & 17.75 & 17.63 & 17.57 & 17.75 & 17.76 & 17.77 & 16.95 & 1.31 \\
\hline AEO84 & 18.22 & 18.07 & 18.33 & 18.61 & 18.73 & 18.76 & 18.75 & 1.06 \\
\hline AEO85 & 17.79 & 17.80 & 17.89 & 18.30 & 18.58 & 18.71 & 18.81 & 0.86 \\
\hline AEO 86 & & 16.52 & 16.83 & 17.35 & 17.27 & 17.50 & 18.04 & 1.28 \\
\hline AEO $87 \ldots$. & & & 16.85 & 16.93 & 17.24 & 17.27 & 18.31 & 1.55 \\
\hline AEO89 $\ldots \ldots \ldots$ & & & & 17.75 & 17.95 & 17.94 & 18.94 & 1.14 \\
\hline AEO90 & & & & & 18.34 & 18.66 & 20.69 & 0.47 \\
\hline AEO91 & & & & & & 18.53 & 20.01 & 0.89 \\
\hline AEO92 & & & & & & & 20.53 & 1.07 \\
\hline AEO93 $\ldots \ldots \ldots \ldots$ & & & & & & & 20.97 & 0.63 \\
\hline Actual & 17.28 & 16.22 & 17.21 & 18.03 & 18.80 & 18.72 & 21.60 & \\
\hline \multirow{2}{*}{$\begin{array}{l}\text { Average Absolute } \\
\text { Error } \ldots \ldots \ldots \ldots \ldots\end{array}$} & 0.83 & 1.13 & 0.73 & 0.73 & 1.00 & 0.70 & 2.40 & 1.16 \\
\hline & \multicolumn{7}{|c|}{ (percent error) } & \\
\hline AEO 82 & -8.0 & -3.1 & -8.7 & -10.7 & -11.9 & -8.8 & & 8.5 \\
\hline AEO83 & 2.7 & 8.7 & 2.1 & -1.6 & -5.5 & -5.1 & -21.5 & 6.7 \\
\hline AEOB4 & 5.4 & 11.4 & 6.5 & 3.2 & -0.4 & 0.2 & -13.2 & 5.8 \\
\hline AEO85 & 2.9 & 9.7 & 3.9 & 1.5 & -1.2 & 0.0 & -12.9 & 4.6 \\
\hline AEO86 $\ldots \ldots \ldots$ & & 1.8 & -2.2 & -3.8 & -8.1 & -6.5 & -16.5 & 6.5 \\
\hline AEO87 & & & -2.1 & -6.1 & -8.3 & -7.7 & -15.2 & 7.9 \\
\hline AEOB9 & & & & -1.6 & -4.5 & -4.1 & -12.3 & 5.6 \\
\hline AEO90 $\ldots \ldots \ldots$ & & & & & -2.5 & -0.3 & -4.2 & 2.3 \\
\hline AEO91 $\ldots \ldots \ldots \ldots$ & & & & & & -1.0 & -7.3 & 4.2 \\
\hline AEO92 & & & & & & & -4.9 & 4.9 \\
\hline AEO93 & & & & & & & -2.9 & 2.9 \\
\hline \multicolumn{9}{|l|}{ Average Absolute } \\
\hline Percent Error $\ldots \ldots \ldots$ & 4.8 & 6.9 & 4.3 & 4.1 & 5.3 & 3.8 & 11.1 & 6.0 \\
\hline
\end{tabular}

AEO = Annual Energy Outlook.

Notes: AEO82 projections given in British thermal units; converted to trillion cubic feet using conversion factors provided in the Monthly Energy Review. Data for 1995 are preliminary.

Sources: History: Energy Information Administration (EIA), Monthly Energy Review, DOE/EIA-0035 (96/03) (Washington, DC, March 1996). Projections: EIA, various issues, Annual Energy Outlook, DOE/EIA-0383(82-93) (Washington, DC, April 1983 through January 1993).

$1993^{13}$ (AEO93) underpredicted consumption by anywhere from 3 to 22 percent. In the case of 1995, price forecasts were one important cause for the underprediction, as prices were overstated considerably in comparison to the actual 1995 price. Another important reason was the underestimation of the penetration of new gas technologies in the end-use and electricity sectors, leading to increased natural gas consumption.
With the exceptions of the projections for 1985 through 1987 made in the AEO83 through AEO85 publications and the $A E O 82$ forecasts, natural gas consumption was generally underpredicted, concurrent with high price projections. Where consumption was overestimated, the indication is that the tendency to conservation and the impact of higher prices on demand were not fully captured, since prices were generally overestimated as well.

\footnotetext{
${ }^{13}$ Energy Information Administration, Annual Energy Outlook 1993, DOE/EIA-0383(93) (Washington, DC, January 1993).
} 
Table 5. Total Coal Consumption, Actual vs. Forecasts

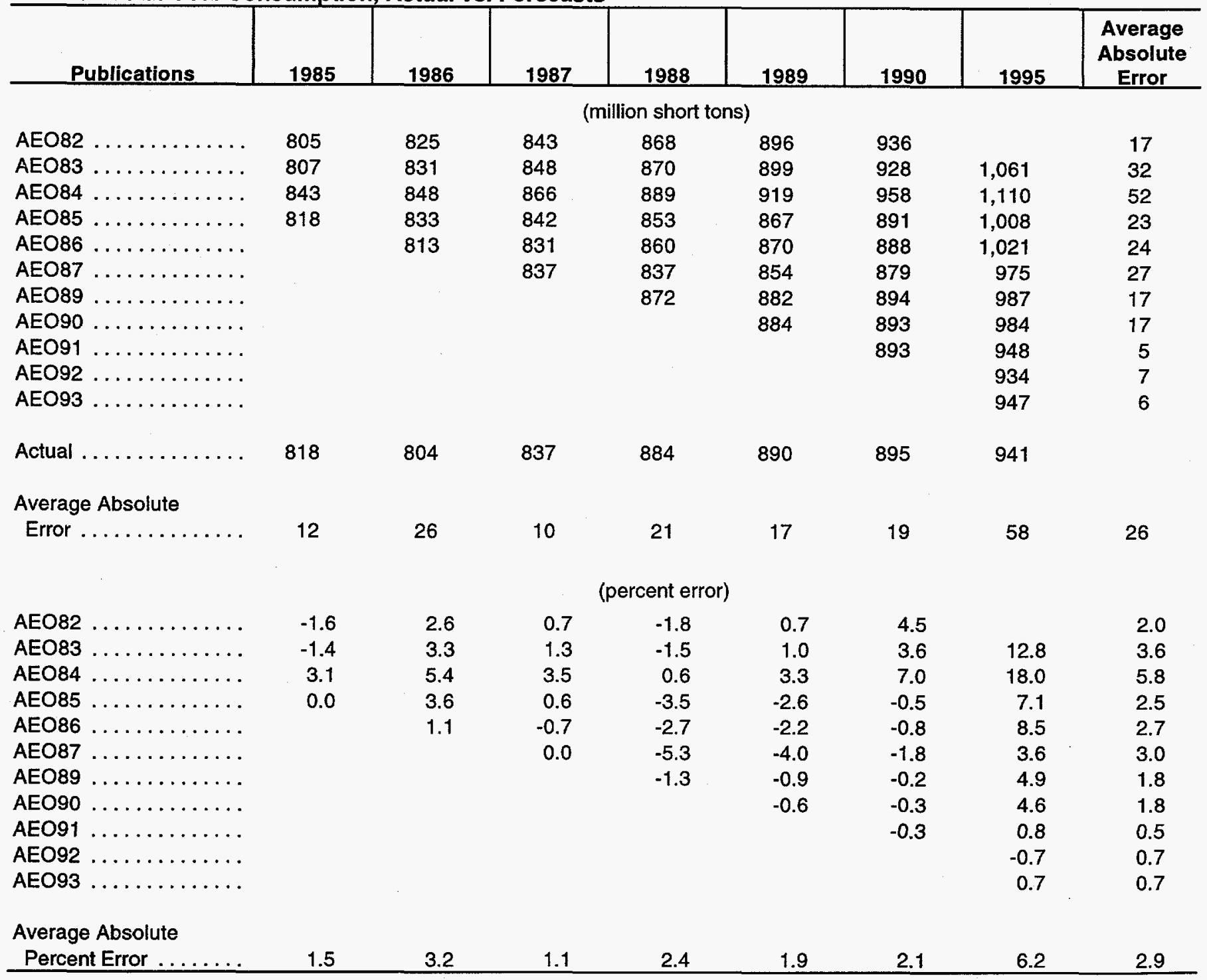

AEO = Annual Energy Outlook.

Notes: Data for 1995 are preliminary.

Sources: History: Energy Information Administration (EIA), Monthly Energy Review, DOE/EIA-0035 (96/03) (Washington, DC, March 1996). Projections: ElA, various issues, Annual Energy Outlook, DOE/EIA-0383(82-93) (Washington, DC, April 1983 through January 1993).

Among the $A E O s$, overall errors ranged from 2 to almost 9 percent. The AEO82 had the highest overall error, due mainly to its underestimate of natural gas use in electricity generation. The largest error in that forecast was for 1989, a 12-percent underprediction. As the later projections did, the AEO82 underpredicted consumption for all years, with high price forecasts contributing to the errors.

\section{Total Coal Consumption}

The forecasts for coal consumption have been stable and displayed fairly low average errors, in part due to the good record in forecasting electricity sales, for which coal is a major component in the production. The overall error for coal consumption is 2.9 percent (Table 5 ). As has generally been the case, forecasts for the year 1995 tend to have the highest error, averaging just over 6 percent. There was a strong tendency to overestimate in the earlier $A E O$, particularly the $A E O 84$, whose forecast was 18 percent over actual 1995 coal consumption. Factors contributing to the overestimate included a 6-percent overestimate for electricity sales, an efficiency estimate that was about 5 percent too low for coal-fired generating units, and a share for coal in generation that did not account for the eventual 
greater role for natural gas, particularly among nonutility electricity producers. The shares of coal and natural gas in the industrial sector were similarly affected, with high natural gas price forecasts and an overly optimistic view of the future of metallurgical coal in steelmaking being the primary factors.

Because of the high 1995 projection, the AEO84 had the highest error for coal consumption of the AEOs examined, at 5.8 percent. All of the others had average errors no higher than 3.6 percent.

\section{Total Electricity Sales}

The average error for projections for this variable is 1.8 percent over the period studied (Table 6). Although 1995 had the highest average error- -2.3 percent-forecasts for that year are not greatly out of line with those of the earlier years, reflecting the general stability in electricity sales over time. Although there was overpredicting of 1995 in the earlier $A E O$ s, more recent $A E O s$ - the Annual Energy Outlook 1991 $1^{14}$ (AEO91), the Annual Energy Outlook $1992^{15}$ (AEO92), and AEO93-have tended to underestimate 1995. In fact, most of the forecasts for electricity sales after the $A E O 84$ have underpredicted most years. This is primarily due to overly optimistic estimates of efficiency improvements, coupled with new uses for electricity that were not captured in the projections. In addition, price forecasts have tended to be overstated in most years, in part due to the influence of overstated natural gas and coal prices to electricity producers.

In terms of the $A E O$ publications, the highest average error was that of the AEO82, at 2.7 percent. All of the AEO82 projections were above the eventual actual levels of sales, indicating that the models used in that $A E O$ continued to anticipate electricity growth at a pace near that of economic growth, a ratio that has actually been reduced considerably in this decade. The next worst forecast record was for the $A E O 86$, primarily due to underpredictions for the years 1988 through 1990.

\section{Energy Production}

\section{Crude Oil Production}

Crude oil production forecasts have been generally accurate, with an overall average error of 5 percent over the period evaluated (Table 7). The largest error for any given year was 1989, with an average error of almost 8 percent and all $A E O$ s overestimating actual production for that year. Since domestic oil production is presumed to be determined by prices rather than demand, an important input to production forecasts is the world oil price, which has also been overestimated in most years, particularly for the $A E O 82$ through $A E O 85$ projections. For 1989, the first four $A E O$ s had significantly high world oil price projections, leading to high production forecasts. Following the $A E O 85$, EIA's price forecasts were either very close, or significantly under, the actual 1989 price, with a consequent improvement in production projections.

Each of the AEOs has had average errors for crude oil production at 7 percent or lower, with the exception of $A E O 83$, which had an average error of 10 percent. AEO83 tended to overpredict crude oil production for all years after 1985, with particularly large errors for 1989, 1990, and 1995 (24 percent). High price forecasts are the primary reason for the errors.

EIA's forecasts of crude oil production have not tended to be understated, in part because of high price forecasts. But even as price projections have been reduced over time, the forecasts have not overreacted, indicating that technological improvement in the oil industry has been fairly well captured by the $A E O$ projections.

\section{Natural Gas Production}

The overall average error for natural gas production forecasts is 5.4 percent, just under the average error for consumption forecasts (Table 8). Unlike crude oil, most demand for natural gas is met by domestic supply sources, so natural gas production tends to follow the projections for consumption. Forecasts for 1995 display the highest average error, at just under 8 percent. The highest error for 1995 occurred in the $A E O 83$, the first $A E O$ to forecast 1995 production. Despite a very high price forecast, the $A E O 83$ production projection was about 20 percent below actual, in line with the low demand projection. The failure to anticipate the amount of gas that would be available at a given price (in part due to the technological improvement impact) led to the high price and low demand and production forecasts in that $A E O$.

With the exception of $A E O 82$, the average error rate for the $A E O$ s has been under 7 percent. The AEO82 underpredicted all years and had an 11.8-percent average error.

\footnotetext{
${ }^{14}$ Energy Information Administration, Annual Energy Outlook 1991, DOE/EIA-0383(91) (Washington, DC, March 1991).

${ }^{15}$ Energy Information Administration, Annual Energy Outlook 1992, DOE/EIA-0383(92) (Washington, DC, January 1992).
} 
Table 6. Total Electricity Sales, Actual vs. Forecasts

\begin{tabular}{|c|c|c|c|c|c|c|c|c|}
\hline Publications & 1985 & 1986 & 1987 & 1988 & 1989 & 1990 & 1995 & $\begin{array}{c}\text { Average } \\
\text { Absolute } \\
\text { Error }\end{array}$ \\
\hline & \multicolumn{8}{|c|}{ (billion kilowatthours) } \\
\hline AEO82 & 2,364 & 2,454 & 2,534 & 2,626 & 2,708 & 2,811 & & 68 \\
\hline AEO83 & 2,318 & 2,395 & 2,476 & 2,565 & 2,650 & 2,739 & 3,153 & 34 \\
\hline AEO84 & 2,321 & 2,376 & 2,461 & 2,551 & 2,637 & 2,738 & 3,182 & 36 \\
\hline AEO85 & 2,317 & 2,360 & 2,427 & 2,491 & 2,570 & 2,651 & 3,026 & 42 \\
\hline AEO86 & & 2,363 & 2,416 & 2,479 & 2,533 & 2,608 & 3,048 & 68 \\
\hline AEO87 & & & 2,460 & 2,494 & 2,555 & 2,622 & 2,977 & 60 \\
\hline AEO89 & & & & 2,556 & 2,619 & 2,689 & 3,072 & 35 \\
\hline AEO90 & & & & & 2,612 & 2,689 & 3,083 & 46 \\
\hline AEO91 & & & & & & 2,700 & 2,959 & 29 \\
\hline AEO92 & & & & & & & 2,975 & 30 \\
\hline AEO93 $\ldots \ldots \ldots$ & & & & & & & 2,946 & 59 \\
\hline Actual & 2,324 & 2,369 & 2,457 & 2,578 & 2,647 & 2,713 & 3,005 & \\
\hline \multicolumn{9}{|l|}{ Average Absolute } \\
\hline Error...$\ldots \ldots \ldots$ & 14 & 27 & 29 & 54 & 53 & 52 & 70 & 48 \\
\hline & \multicolumn{8}{|c|}{ (percent error) } \\
\hline AEO82 & 1.7 & 3.6 & 3.1 & 1.9 & 2.3 & 3.6 & & 2.7 \\
\hline AEO83 & -0.3 & 1.1 & 0.8 & -0.5 & 0.1 & 1.0 & 4.9 & 1.2 \\
\hline AEO84 & -0.1 & 0.3 & 0.2 & -1.0 & -0.4 & 0.9 & 5.9 & 1.3 \\
\hline AEO85 $\ldots$ & -0.3 & -0.4 & -1.2 & -3.4 & -2.9 & -2.3 & 0.7 & 1.6 \\
\hline AEO86 $\ldots \ldots \ldots \ldots$ & & -0.3 & -1.7 & -3.8 & -4.3 & -3.9 & 1.4 & 2.6 \\
\hline AE087 & & & 0.1 & -3.3 & -3.5 & -3.4 & -0.9 & 2.2 \\
\hline AEO89 & & & & -0.9 & -1.1 & -0.9 & 2.2 & 1.3 \\
\hline AEO90 $\ldots \ldots \ldots \ldots$ & & & & & -1.3 & -0.9 & 2.6 & 1.6 \\
\hline AEO91 $\ldots \ldots \ldots$ & & & & & & -0.5 & -1.5 & 1.0 \\
\hline AEO92 $\ldots \ldots \ldots$ & & & & & & & -1.0 & 1.0 \\
\hline AEO93 $\ldots \ldots \ldots \ldots$ & & & & & & & -2.0 & 2.0 \\
\hline \multicolumn{9}{|l|}{ Average Absolute } \\
\hline Percent Error & 0.6 & 1.1 & 1.2 & 2.1 & 2.0 & 1.9 & 2.3 & 1.8 \\
\hline
\end{tabular}

AEO = Annual Energy Outlook.

Notes: Data for 1995 are preliminary.

Sources: History: Energy Information Administration (EIA), Monthly Energy Review, DOE/EIA-0035 (96/03) (Washington, DC, March 1996). Projections: EIA, various issues, Annual Energy Outlook, DOE/EIA-0383(82-93) Washington, DC, April 1983 through January 1993).

Again, the error in production derived from the low forecast for consumption in each of the years, concurrent with price forecasts that were quite high; the conclusion is that, at least in part, improvements in technology were not well-captured in that $A E O$. In general, the $A E O$ s have understated production, with the exception of the years prior to 1990 in the AEO84 and AEO85, and most of the errors have been very similar to those for forecasts for natural gas consumption.

\section{Coal Production}

Similar to coal consumption, coal production forecasts have shown a good record, with an overall average error of less than 4 percent (Table 9). Similar to natural gas, the forecasts for coal production have generally followed those for consumption, with electricity sales being the dominant factor. However, an additional input is the level of coal exports, which also affects coal production significantly. 
Table 7. Crude Oil Production, Actual vs. Forecasts

\begin{tabular}{|c|c|c|c|c|c|c|c|c|}
\hline Publications & 1985 & 1986 & 1987 & 1988 & 1989 & 1990 & 1995 & $\begin{array}{c}\text { Average } \\
\text { Absolute } \\
\text { Error }\end{array}$ \\
\hline \multicolumn{9}{|c|}{ (million barrels per day) } \\
\hline$\ldots \ldots \ldots$ & 8.79 & 8.85 & 8.84 & 8.80 & 8.66 & 8.21 & & 0.57 \\
\hline$\ldots \ldots \ldots \ldots$ & 8.67 & 8.71 & 8.66 & 8.72 & 8.80 & 8.63 & 8.11 & 0.75 \\
\hline$\ldots \ldots \ldots \ldots$ & 8.86 & 8.70 & 8.59 & 8.45 & 8.28 & 8.25 & 7.19 & 0.41 \\
\hline$\ldots \ldots \ldots \ldots$ & 8.92 & 8.96 & 9.01 & 8.78 & 8.38 & 8.05 & 6.53 & 0.44 \\
\hline AEO86 & & 8.80 & 8.63 & 8.30 & 7.90 & 7.43 & 5.99 & 0.24 \\
\hline AEO $87 \ldots \ldots \ldots \ldots$ & & & 8.31 & 8.18 & 8.00 & 7.63 & 6.54 & 0.15 \\
\hline AEO89 $\ldots \ldots \ldots \ldots$ & & & & 8.18 & 7.97 & 7.64 & 6.17 & 0.26 \\
\hline AEO90 $\ldots \ldots \ldots \ldots$ & & & & & 7.67 & 7.37 & 6.40 & 0.07 \\
\hline AEO91 $\ldots \ldots \ldots \ldots$ & & & & & & 7.23 & 6.79 & 0.19 \\
\hline$\ldots \ldots \ldots \ldots$ & & & & & & & 6.68 & 0.15 \\
\hline$\ldots \ldots \ldots \ldots$ & & & & & & & 6.52 & 0.01 \\
\hline Actual & 8.97 & 8.68 & 8.35 & 8.14 & 7.61 & 7.36 & 6.53 & \\
\hline \multicolumn{9}{|l|}{ Average Absolute } \\
\hline Error .......... & 0.16 & 0.12 & 0.34 & 0.35 & 0.59 & 0.50 & 0.37 & 0.38 \\
\hline \multicolumn{9}{|c|}{ (percent error) } \\
\hline AEO82 & -2.0 & 2.0 & 5.9 & 8.1 & 13.8 & 11.6 & & 7.2 \\
\hline AEO83 & -3.4 & 0.3 & 3.7 & 7.1 & 15.6 & 17.3 & 24.2 & 10.2 \\
\hline AE084 & -1.2 & 0.2 & 2.9 & 3.8 & 8.8 & 12.2 & 10.1 & 5.6 \\
\hline AEO85 & -0.6 & 3.2 & 7.9 & 7.9 & 10.1 & 9.4 & 0.0 & 5.6 \\
\hline AEO86 $\ldots \ldots \ldots$ & & 1.4 & 3.4 & 2.0 & 3.8 & 1.0 & -8.3 & 3.3 \\
\hline AE087 & & & -0.5 & 0.5 & 5.1 & 3.7 & 0.2 & 2.0 \\
\hline AEO89 & & & & 0.5 & 4.7 & 3.9 & -5.5 & 3.6 \\
\hline AEO90 $\ldots \ldots \ldots$ & & & & & 0.7 & 0.2 & -2.0 & 1.0 \\
\hline AEO91 $\ldots \ldots \ldots$ & & & & & & -1.7 & 4.0 & 2.8 \\
\hline AEO92 $\ldots \ldots \ldots$ & & & & & & & 2.3 & 2.3 \\
\hline AEO93 $\ldots \ldots \ldots \ldots$ & & & & & & & -0.2 & 0.2 \\
\hline \multicolumn{9}{|l|}{ Average Absolute } \\
\hline Percent Error .. & 1.8 & 1.4 & 4.0 & 4.3 & 7.8 & 6.8 & 5.7 & 5.1 \\
\hline
\end{tabular}

$\mathrm{AEO}=$ Annual Energy Outlook.

Notes: Data for 1995 are preliminary.

Sources: History: Energy Information Administration (EIA), Monthly Energy Review, DOE/EIA-0035 (96/03) (Washington, DC, March 1996). Projections: ElA, various issues, Annual Energy Outlook, DOE/EIA-0383(82-93) (Washington, DC, April 1983 through January 1993).

Where coal production has been overestimated, a large part of the reason has been an overstating of the level of coal exports, especially for the year 1995, which is discussed later.

The year 1995 shows the highest average error, at 7.4 percent. Although recent forecasts in $A E O 91$ through $A E O 93$ have shown errors under 4 percent, the forecasts in $A E O 83$ through $A E O 86$ ranged from 8 to 19 percent above the actual 1995 level. This reflects the overprediction of coal consumption in the $A E O 83$ and $A E O 84$ and the higherthan-realized coal export projections in all four $A E O s$, which are discussed later. Other forecasted years averaged much closer to actual, with errors ranging from 2 to 4 percent. The $A E O$ publications display little variation in the overall average error of each, with the AEO84 showing the highest average error of 5 percent, mainly because of its very high projection for 1995. 
Table 8. Natural Gas Production, Actual vs. Forecasts

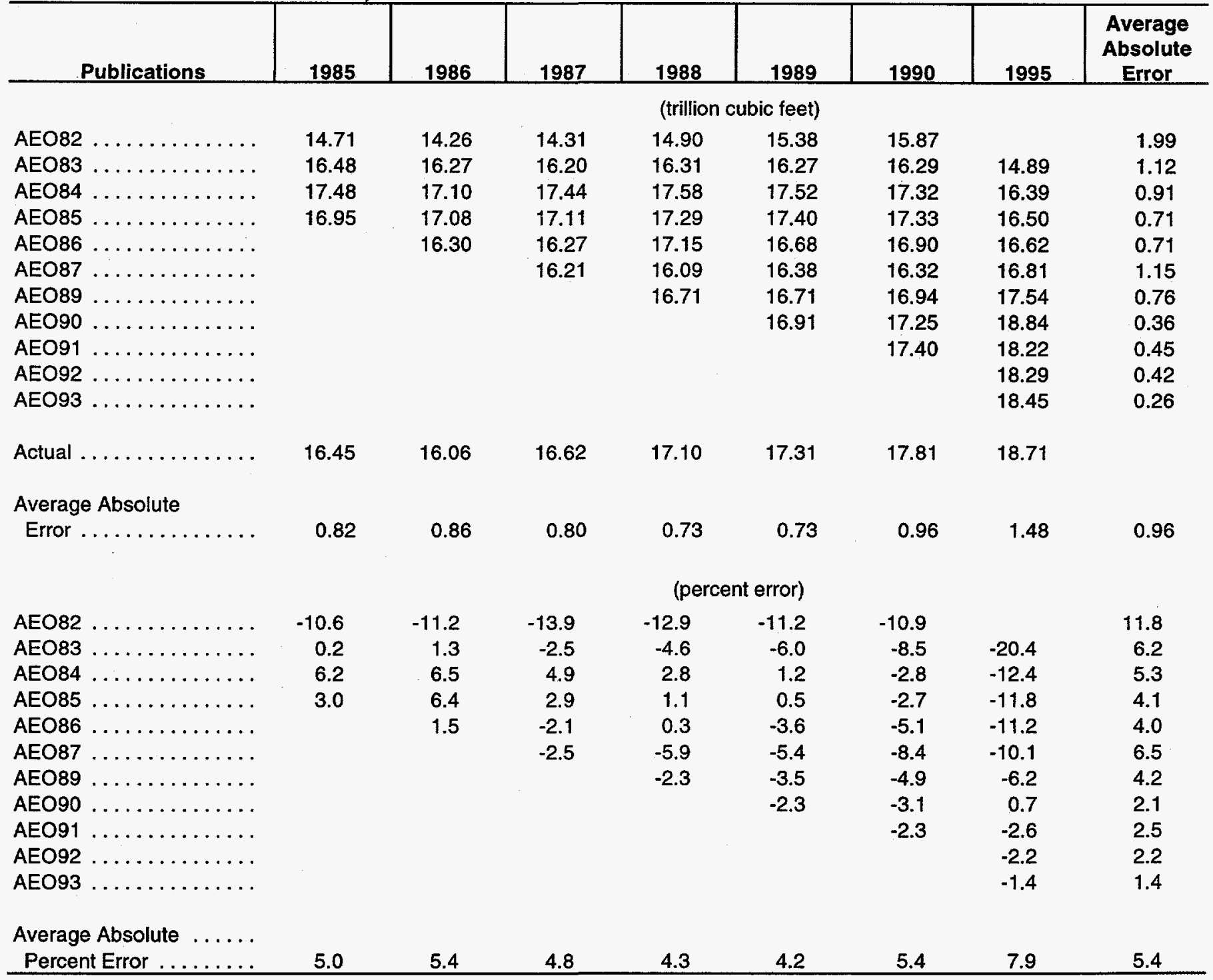

AEO = Annual Energy Outlook.

Notes: AEO82 projections given in British thermal units; converted to trillion cubic feet using conversion factors provided in Monthly Energy Review. Data for 1995 are preliminary.

Sources: History: Energy Information Administration (EIA), Monthly Energy Review, DOE/EIA-0035 (96/03) (Washington, DC, March 1996). Projections: EIA, various issues, Annual Energy Outlook, DOE/EIA-0383(82-93) (Washington, DC, April 1983 through January 1993).

\section{Energy Imports and Exports}

While the United States is a major importer of petroleum, it also imports natural gas, although in much smaller quantities. Coal is the only fuel for which the United States is a net exporter.

\section{Net Petroleum Imports}

Since domestic production of crude oil is insufficient to meet the demand for petroleum, imports make up the difference between demand and supply. ${ }^{16}$ The average error for net petroleum imports over the period studied

\footnotetext{
${ }^{16}$ Stocks may also contribute but are assumed to be stable over the long term and have not been specifically projected in the $A E O$ forecasts.
} 
Table 9. Coal Production, Actual vs. Forecasts

\begin{tabular}{|c|c|c|c|c|c|c|c|c|}
\hline Publications & 1985 & 1986 & 1987 & 1988 & 1989 & 1990 & 1995 & $\begin{array}{c}\text { Average } \\
\text { Absolute } \\
\text { Error }\end{array}$ \\
\hline \multicolumn{9}{|c|}{ (million short tons) } \\
\hline AEO82 & 914 & 939 & 963 & 995 & 1,031 & 1,080 & & 45 \\
\hline AEO83 & 900 & 926 & 947 & 974 & 1,010 & 1,045 & 1,191 & 44 \\
\hline AEO84 & 899 & 921 & 948 & 974 & 1,010 & 1,057 & 1,221 & 50 \\
\hline AEO85 & 886 & 909 & 930 & 940 & 958 & 985 & 1,116 & 28 \\
\hline AEO86 & & 890 & 920 & 954 & 962 & 983 & 1,126 & 28 \\
\hline AEO87 & & & 917 & 914 & 932 & 962 & 1,068 & 38 \\
\hline AEO89 & & & & 941 & 946 & 977 & 1,082 & 37 \\
\hline AEO90 & & & & & 973 & 987 & 1,085 & 35 \\
\hline AEO91 & & & & & & 1,035 & 1,054 & 15 \\
\hline AEO92 & & & & & & & 1,052 & 22 \\
\hline AEO93 & & & & & & & 1,065 & 35 \\
\hline Actual & 884 & 890 & 919 & 951 & 981 & 1,029 & 1,030 & \\
\hline \multicolumn{9}{|l|}{ Average Absolute } \\
\hline Error. & 16 & 27 & 19 & 22 & 30 & 39 & 76 & 37 \\
\hline \multicolumn{9}{|c|}{ (percent error) } \\
\hline AEO82 & 3.4 & 5.5 & 4.8 & 4.7 & 5.1 & 4.9 & & 4.7 \\
\hline AEO83 & 1.9 & 4.0 & 3.1 & 2.5 & 3.0 & 1.5 & 15.7 & 4.5 \\
\hline AEO84 & 1.7 & 3.4 & 3.2 & 2.5 & 3.0 & 2.7 & 18.6 & 5.0 \\
\hline AEO85 & 0.3 & 2.1 & 1.2 & -1.1 & -2.3 & -4.3 & 8.4 & 2.8 \\
\hline AEO86 & & 0.0 & 0.1 & 0.4 & -1.9 & -4.5 & 9.3 & 2.7 \\
\hline AEO87 & . & & -0.2 & -3.9 & -5.0 & -6.5 & 3.7 & 3.8 \\
\hline AEO89 & & & & -1.0 & -3.5 & -5.1 & 5.1 & 3.7 \\
\hline AEO90 & & & & & -0.8 & -4.1 & 5.4 & 3.4 \\
\hline AEO91 & & & & & & 0.6 & 2.4 & 1.5 \\
\hline AEO92 & & & & & & & 2.2 & 2.2 \\
\hline AEO93 & & & & & & & 3.4 & 3.4 \\
\hline \multicolumn{9}{|l|}{ Average Absolute } \\
\hline Percent Error & 1.8 & 3.0 & 2.1 & 2.3 & 3.1 & 3.8 & 7.4 & 3.8 \\
\hline
\end{tabular}

AEO = Annual Energy Outlook .

Notes: Data for 1995 are preliminary.

Sources: Historical data: Energy Information Administration (EIA), Monthly Energy Review, DOE/EIA-0035 (96/03) (Washington, DC, March 1996). Forecasts: EIA, various issues, Annual Energy Outlook, DOE/EIA-0383(82-93) (Washington, DC, April 1983 through January 1993).

was 12 percent (Table 10). The forecast year with the highest average error proved to be 1985 , for which the $A E O$ s averaged a 28-percent error; subsequent years showed considerable improvement. In general, there was a tendency to underpredict imports for the mid-1980s, stemming from the underprediction of consumption and the overestimates of production. Except for the AEO83, this tendency was reversed for the year 1995, with significant overestimates of net petroleum imports in the AEO84, the AEO87, the Annual Energy Outlook $1989^{17}$
(AEO89), the Annual Energy Outlook $1990^{18}$ (AEO90), and the AEO93.

While in some $A E O$ s this corresponded to overestimates of consumption and/or underestimates of production, it was also exacerbated by the contribution of inaccurate forecasts for other sources of supply (natural gas liquids and processing gain), underestimates of the contribution of stocks, and assumptions about the pace of acquisition of crude oil for the Strategic Petroleum Reserve.

\footnotetext{
${ }^{17}$ Energy Information Administration, Annual Energy Outlook 1989, DOE/EIA-0383(89) (Washington, DC, January 1989).

${ }^{18}$ Energy Information Administration, Annual Energy Outlook 1990, DOE/EIA-0383(90) (Washington, DC, January 1990).
} 
Table 10. Net Petroleum Imports, Actual vs. Forecasts

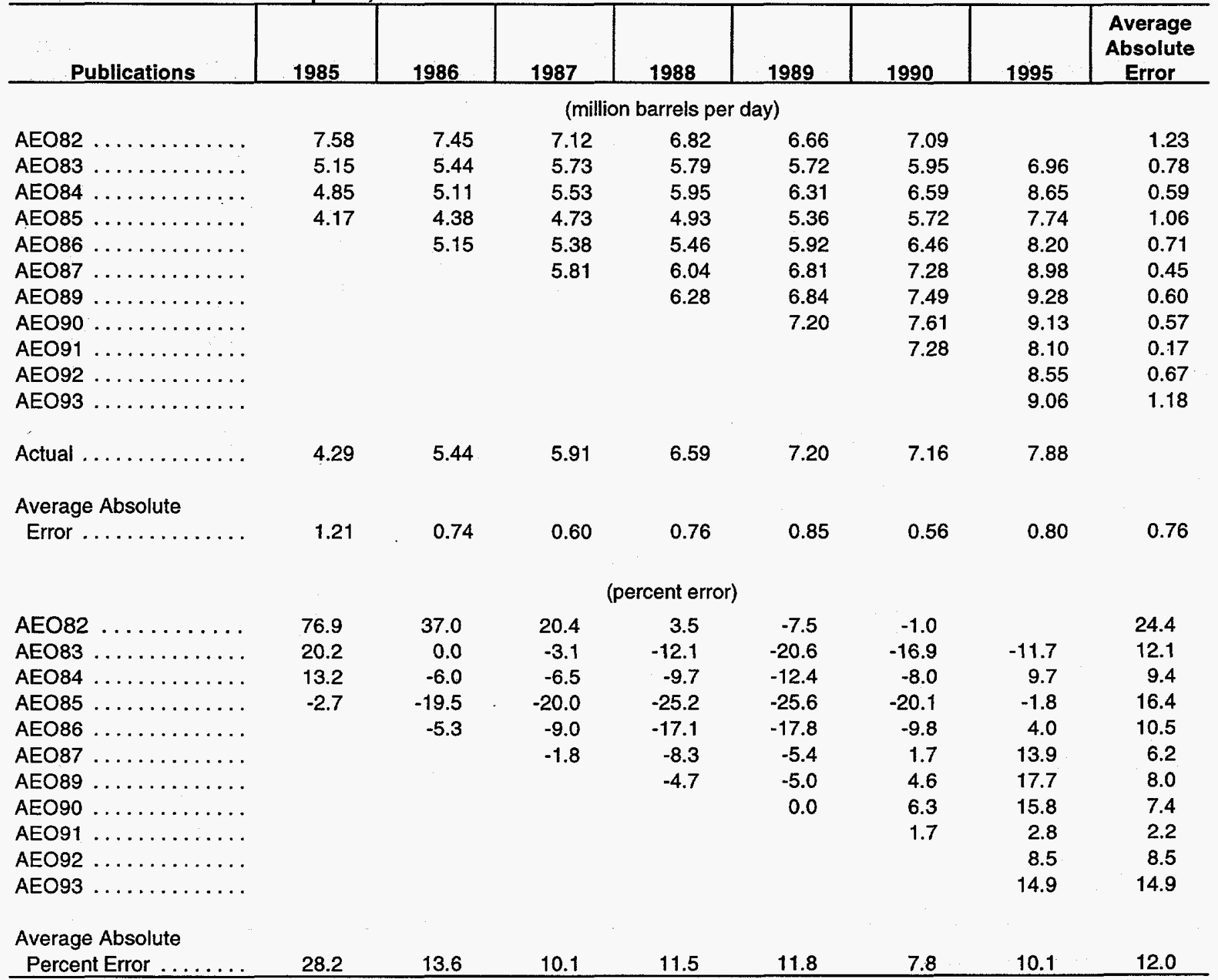

AEO = Annual Energy Outlook.

Notes: Data for 1995 are preliminary.

Sources: History: Energy Information Administration (EIA), Monthly Energy Review, DOE/EIA-0035 (96/03) (Washington, DC, March 1996). Projections: EIA, various issues, Annual Energy Outlook, DOE/EIA-0383(82-93) (Washington, DC, April 1983 through January 1993).

By publication, the $A E O$ s for $1982,1983,1985,1986$, and 1993 proved to have the highest average errors for forecasts of net petroleum imports. The AEO82 tended to strongly overpredict imports for 1985 through 1987; however, its record is surprisingly good for the years 1988 through 1990. The AEO83 did a good job forecasting 1986 and 1987 but strongly underestimated subsequent years, as production forecasts tended to be too high due to higher-than-actual prices. The AEO85 also strongly underpredicted imports, especially for the years from 1986 through 1990, largely for the same reasons.

\section{Net Natural Gas Imports}

Net natural gas imports play a small, but important, supplementary role in meeting natural gas demand. The overall average error for the period covered in this study is 20 percent, with the largest errors for the forecast years 1986, 1987, and 1995 (Table 11). Forecasts for 1986 were all overstated, with errors as high as 80 percent in the AEO82. There was a substantial oil price collapse in 1986, and petroleum imports displaced other energy sources, such as Canadian gas, for much of the Nation's 
Table 11. Net Natural Gas Imports, Actual vs. Forecasts

\begin{tabular}{|c|c|c|c|c|c|c|c|c|}
\hline Publications & 1985 & 1986 & 1987 & 1988 & 1989 & 1990 & 1995 & $\begin{array}{c}\text { Average } \\
\text { Absolute } \\
\text { Error }\end{array}$ \\
\hline \multicolumn{9}{|c|}{ (trillion cubic feet) } \\
\hline AEO $82 \ldots \ldots \ldots \ldots$ & 1.23 & 1.23 & 1.23 & 1.23 & 1.23 & 1.22 & & 0.24 \\
\hline AEO83 $\ldots \ldots \ldots \ldots \ldots$ & 1.08 & 1.16 & 1.23 & 1.23 & 1.23 & 1.23 & 1.23 & 0.37 \\
\hline AEO84 $\ldots \ldots \ldots \ldots$ & 0.99 & 1.05 & 1.16 & 1.27 & 1.43 & 1.57 & 2.11 & 0.21 \\
\hline AEO $87 \ldots \ldots \ldots \ldots$ & & & 0.84 & 0.89 & 1.07 & 1.16 & 1.75 & 0.35 \\
\hline AEO89 $\ldots \ldots \ldots \ldots$ & & & & 1.15 & 1.32 & 1.44 & 1.87 & 0.21 \\
\hline AEO90 . . . . . . . & & & & & 1.26 & 1.43 & 2.07 & 0.18 \\
\hline AEO9 $1 \ldots \ldots \ldots \ldots$ & & & & & & 1.36 & 2.30 & 0.18 \\
\hline AEO92 $\ldots \ldots \ldots \ldots$ & & & & & & & 2.25 & 0.32 \\
\hline \multicolumn{9}{|l|}{ Average Absolute } \\
\hline Error...$\ldots \ldots \ldots$ & 0.16 & 0.35 & 0.20 & 0.19 & 0.13 & 0.20 & 0.54 & 0.27 \\
\hline \multicolumn{9}{|c|}{ (percent error) } \\
\hline AEO $82 \ldots \ldots \ldots \ldots$ & 37.2 & 79.1 & 31.1 & 0.6 & -3.9 & -15.9 & & 28.0 \\
\hline AEO $83 \ldots \ldots \ldots \ldots$ & 20.7 & 68.4 & 31.0 & 0.8 & -3.5 & -14.9 & -52.2 & 27.4 \\
\hline AEO $84 \ldots \ldots \ldots \ldots$ & 10.6 & 52.4 & 23.5 & 4.1 & 12.2 & 8.6 & -18.0 & 18.5 \\
\hline AEO85 $\ldots \ldots \ldots \ldots$ & 5.0 & 45.1 & 26.7 & 18.9 & 23.9 & 28.6 & -5.2 & 21.9 \\
\hline AEO86 $\ldots \ldots \ldots \ldots$ & & 7.4 & -6.3 & -49.2 & -19.2 & -27.4 & -30.5 & 23.3 \\
\hline AEO $87 \ldots \ldots \ldots \ldots$ & & & -10.5 & -27.0 & -16.1 & -19.8 & -32.0 & 21.1 \\
\hline \multicolumn{9}{|l|}{ Average Absolute } \\
\hline Percent Error . . . . . . & 18.4 & 50.5 & 21.5 & 15.2 & 10.4 & 13.6 & 21.1 & 20.0 \\
\hline
\end{tabular}

AEO = Annual Energy Outlook

Notes: AEO82 projections given in British thermal units; converted to trillion cubic feet using conversion factors provided in the Monthly Energy Review. Data for 1995 are preliminary.

Sources: History: Energy Information Administration (EIA), Monthly Energy Review, DOE/ElA-0035 (96/03) (Washington, DC, March 1996). Projections: EIA, various issues, Annual Energy Outlook, DOE/EIA-0383(82-93) (Washington, DC, April 1983 through January 1993).

consumption needs, especially in the industrial and electric generation sectors. Forecasts for 1987 were overstated in the first four AEOs, but the AEO86 and $A E O 87$ reversed the pattern with underestimates. For 1995 , there was an underestimate in all of the $A E O s$, with errors ranging from 3.3 percent in the $A E O 93$ to over 50 percent in AEO83.
Natural gas imports have generally been projected as offline assumptions in the $A E O$; the major determining factors have been the economics of natural gas trade with Canada and the assumptions concerning gas pipeline capacity. In addition, assessments of liquefied natural gas imports from Algeria and prospects for trade with Mexico and Japan have also affected the projections. The tendency 
was for net gas imports to be overstated for the first four $A E O$ s, except for the 1990 and 1995 forecasts. Since the $A E O 86$ forecast, there has been a greater tendency to underpredict. Although net imports have increased over time, this trend has not always been reflected in the updated forecasts from one $A E O$ to the next, with the exception of forecasts for 1995. Since the AEO87, the projections have been much closer to actual, with the exception of the 1995 forecasts, which continued to be underpredicted by as much as 27 percent, although the forecast for 1995 in the AEO93 underestimated actual by only 3 percent.

\section{Net Coal Exports}

The errors in projections for net coal exports have averaged 17 percent over the period of this study (Table 12). The forecast year 1995 had by far the highest average errors, with all of the $A E O$ s overstating coal exports by anywhere from 6 to 44 percent. Other forecasted years showed a better record, with only an 11-percent average forecast error for 1986. Since the $A E O 82$, coal exports have generally been underestimated, with the exception of 1995.

The AEO82 overestimated future coal exports by an average of 38 percent, due largely to the assumption that U.S. coal exports would garner an ever-increasing share of world coal trade, which would itself grow in reaction to high world oil prices. The AEO83, by contrast, had a much more realistic view of future coal exports and, with the exception of 1995, came closest of all the $A E O$ s with respect to projected coal exports. Projections of more recent $A E O$ s for 1995-AEO92 and $A E O 93$ - were far too high, in part because of the 1993 coal miners' strike that reduced this country's competitive position in world coal markets and because world coal trade has not grown as much as previously assumed, since European consumers have turned increasingly to natural gas for industry and power generation and environmental concerns have led some countries to reduce coal consumption as a means of reducing carbon emissions.

\section{Energy Prices and Disposable Personal Income ${ }^{19}$}

\section{World Oil Prices}

World oil prices have been one of the two variables with the highest average forecast errors of all of those evaluated in this paper, with natural gas prices at the wellhead being the other. Overall, the average percent error for world oil price forecasts has been 82 percent (Table 13). However, the earlier $A E O$ s had a much higher average error, with the publications after the $A E O 85$ showing considerable improvement. Similarly, the year with the highest average forecast errors was 1995, with very high errors in the early $A E O$ s only partially offset by smaller errors in the more recent forecasts. In nominal terms, the first forecast for 1995 appearing in the AEO83 was over $\$ 100$ per barrel, compared to an actual price of $\$ 17.13$ per barrel.

Although the forecasts appearing in the earlier $A E O$ s were almost uniformly too high, from the $A E O 86$ on there were several instances of underprediction. These included the 1987 forecast appearing in the AEO86 and the forecasts for 1989 and 1990 appearing in the AEO89 and AEO90. Clearly, following the oil price collapse of 1986, EIA's forecasts were significantly reduced; as a consequence, the projections for 1990 tended to be too low, in part because of the rise in oil prices beginning in August 1990 associated with Iraq's invasion of Kuwait. Even with the lower price forecasts, 1995 had high average forecast errors, as most $A E O$ s continued to show rising prices in response to perceived rising world oil demand. The best forecast for 1995 appeared in the $A E O 93$, with a projection 30 percent above the actual price.

The early $A E O$ projections were strongly influenced by the notion that OPEC would continue to hold a measure of power in world oil markets. Conventional wisdom in the early projections generally assumed that OPEC would be able to curtail production sufficiently to hold prices up and that the cartel's members would continue their cooperation throughout the forecast horizon.

Even as it became clear that OPEC's cohesiveness was not permanent, ELA continued to assume that oil prices would rise with increasing demand, although at a much slower rate of growth than in the 1970s. However, the impacts of growth in capacity of non-OPEC producers, such as Mexico, the United Kingdom, and the United States (in Alaska); competition from natural gas; and energy conservation have kept prices lower than expected.

\section{Natural Gas Prices}

Natural gas prices at the wellhead have had the highest average forecast errors in the AEOs examined, with an overall average error of 120 percent (Table 14). Only twice

\footnotetext{
${ }^{19}$ Forecasts of energy prices and real disposable personal income (RDPI) have been converted to nominal terms by using the gross national product deflator or the gross domestic product (GDP) deflator given in each AEO. Actual prices and actual RDPI were converted to nominal terms using the GDP deflator. This was done so that the comparisons could be made on a consistent basis.
} 
Table 12. Net Coal Exports, Actual vs. Forecasts

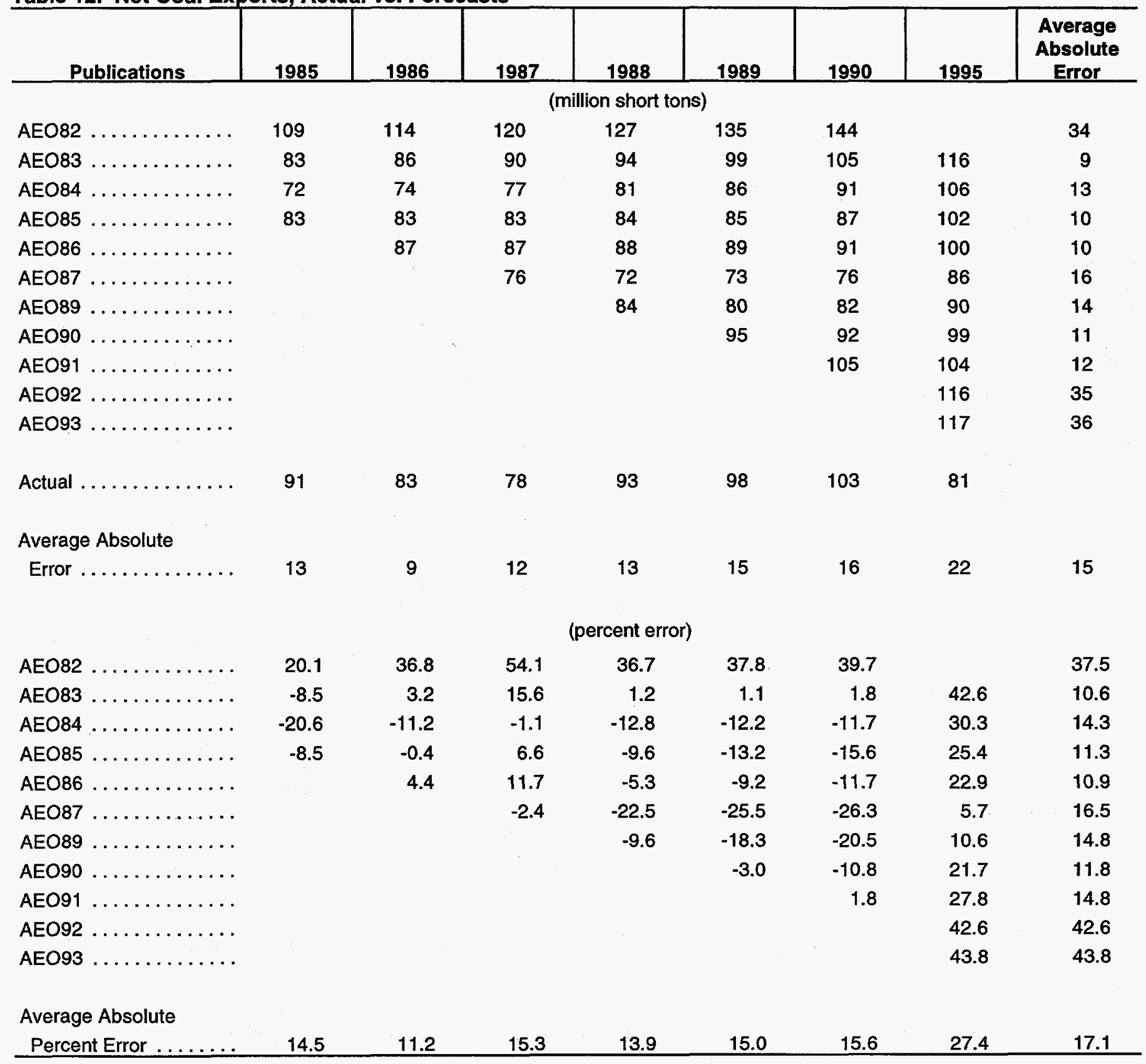

AEO = Annual Energy Outlook.

Notes: Data for 1995 are preliminary.

Sources: History: Energy Information Administration (EIA), Monthly Energy Review, DOE/EIA-0035 (96/03) (Washington, DC, March 1996). Projections: EIA, various issues, Annual Energy Outlook, DOE/EIA-0383(82-93) (Washington, DC, April 1983 through January 1993).

has EIA underestimated gas prices, the most recent being the 1988 forecast appearing in the AEO89. Similar to the world oil price, forecasts for natural gas prices were highest in the earlier $A E O$ s, as the projections for all prices were influenced by the assumption that market forces would tend to increase demand, and therefore prices, of natural gas and coal in response to the higher world oil prices. 
Table 13. World Oil Prices, Actual vs. Forecasts

\begin{tabular}{|c|c|c|c|c|c|c|c|c|}
\hline Publications & 1985 & 1986 & 1987 & 1988 & 1989 & 1990 & 1995 & $\begin{array}{c}\text { Average } \\
\text { Absolute } \\
\text { Error }\end{array}$ \\
\hline \multicolumn{9}{|c|}{ (dollars per barrel) } \\
\hline AEO82 & 29.66 & 34.82 & 41.43 & 48.04 & 53.75 & 59.06 & & 25.54 \\
\hline$\ldots \ldots \ldots \ldots$ & 28.99 & 29.39 & 32.67 & 39.32 & 46.07 & 53.22 & 101.59 & 28.66 \\
\hline AEO84 & 29.07 & 29.39 & 30.97 & 33.96 & 37.23 & 40.82 & 73.87 & 20.66 \\
\hline AEO85 & 27.00 & 25.86 & 24.83 & 26.05 & 29.69 & 33.75 & 49.65 & 12.31 \\
\hline AEO86 & & 14.57 & 15.87 & 17.30 & 18.86 & 20.64 & 39.92 & 5.04 \\
\hline AEOB $7 \ldots \ldots \ldots$ & & & 18.11 & 17.30 & 18.91 & 19.93 & 32.03 & 4.06 \\
\hline$\ldots \ldots \ldots \ldots$ & & & & 14.70 & 14.93 & 16.33 & 28.91 & 5.12 \\
\hline AEO90 $\ldots \ldots$ & & & & & 17.70 & 17.49 & 26.58 & 4.70 \\
\hline AEO91 & & & & & & 22.00 & 28.37 & 5.74 \\
\hline AEO92 & & & & & & & 24.20 & 7.07 \\
\hline AEO93 $\ldots \ldots \ldots \ldots$ & & & & & & & 22.34 & 5.21 \\
\hline Actual & 26.99 & 14.00 & 18.13 & 14.56 & 18.08 & 21.76 & 17.13 & \\
\hline \multicolumn{9}{|l|}{ Average Absolute } \\
\hline \multirow[t]{2}{*}{ Error .......... } & 1.69 & 12.81 & 9.94 & 13.53 & 12.45 & 12.52 & 25.61 & 14.16 \\
\hline & \multicolumn{8}{|c|}{ (percent error) } \\
\hline AEO82 & 9.9 & 148.7 & 128.5 & 229.9 & 197.3 & 171.4 & & 147.6 \\
\hline AEO83 ... & 7.4 & 110.0 & 80.2 & 170.0 & 154.8 & 144.6 & 493.0 & 165.7 \\
\hline AEO84 $\ldots$ & 7.7 & 110.0 & 70.8 & 133.2 & 105.9 & 87.6 & 331.3 & 120.9 \\
\hline AEO85 $\ldots \ldots \ldots \ldots \ldots$ & 0.0 & 84.7 & 36.9 & 78.9 & 64.2 & 55.1 & 189.8 & 72.8 \\
\hline AEO86 $\ldots \ldots \ldots \ldots$ & & 4.1 & -12.5 & 18.8 & 4.3 & -5.2 & 133.0 & 29.6 \\
\hline AE087 $\ldots$ & & & -0.1 & 18.8 & 4.6 & -8.4 & 87.0 & 23.8 \\
\hline AEO89 $\ldots$. & & & & 1.0 & -17.4 & -24.9 & 68.8 & 28.0 \\
\hline AEO90 $\ldots \ldots \ldots$ & & & & & -2.1 & -19.6 & 55.1 & 25.6 \\
\hline AEO91 $\ldots \ldots \ldots \ldots$ & & & & & & 1.1 & 65.6 & 33.3 \\
\hline AEO92 $\ldots \ldots \ldots \ldots$ & & & & & & & 41.3 & 41.3 \\
\hline AEO93 $\ldots \ldots \ldots$ & & & & & & & 30.4 & 30.4 \\
\hline \multicolumn{9}{|l|}{ Average Absolute } \\
\hline Percent Error & 6.3 & 91.5 & 54.8 & 93.0 & 68.8 & 57.5 & 149.5 & 82.2 \\
\hline
\end{tabular}

AEO = Annual Energy Outlook

Notes: Data for 1995 are preliminary.

Sources: History: Energy Information Administration (EIA), Monthly Energy Review, DOE/EIA-0035 (96/03) (Washington, DC, March 1996). Projections: EIA, various issues, Annual Energy Outlook, DOE/EIA-0383(82-93) (Washington, DC, April 1983 through January 1993).

The year 1995 had the highest average forecast errors, with the smallest error, in the $A E O 93$, still above 40 percent. The year with the lowest average errors was 1985 , with an average error for four $A E O$ s of 26 percent, even including the 72-percent error in the AEO82 projection for 1985. Errors for the years between 1985 and 1995 averaged between 67 and 112 percent.

Despite the large errors, the forecasts for each subsequent $A E O$ have tended to show considerable improvement, as the downward trend in gas prices was better captured from one $A E O$ to another. Forecasts for 1990 improved from a 400-percent error in the $A E O 82$ to an error of 3.5 percent in the $A E O 91$. Projections for 1987 improved from 300 percent to under 10 percent.

Nevertheless, each $A E O$ has tended to predict rising prices over time, either because of the assumption in the earlier $A E O$ s that long-term contracts would continue or because the depletion effects associated with rising consumption 
Table 14. Natural Gas Wellhead Prices, Actual vs. Forecasts

\begin{tabular}{|c|c|c|c|c|c|c|c|c|}
\hline Publications & 1985 & 1986 & 1987 & 1988 & 1989 & 1990 & 1995 & $\begin{array}{c}\text { Average } \\
\text { Absolute } \\
\text { Error }\end{array}$ \\
\hline \multicolumn{9}{|c|}{ (dollars per thousand cubic feet) } \\
\hline AEO82 & 4.33 & 5.47 & 6.68 & 7.51 & 8.05 & 8.58 & & 4.90 \\
\hline AEO 83 & 2.93 & 3.11 & 3.46 & 3.93 & 4.56 & 5.26 & 12.74 & 3.31 \\
\hline AEO84 & 2.77 & 2.90 & 3.21 & 3.63 & 4.13 & 4.79 & 9.33 & 2.56 \\
\hline AEO85 $\ldots \ldots$ & 2.60 & 2.61 & 2.66 & 2.71 & 2.94 & 3.35 & 6.67 & 1.53 \\
\hline AEO86 $\ldots \ldots \ldots \ldots \ldots$ & & 1.73 & 1.96 & 2.29 & 2.54 & 2.81 & 5.90 & 1.23 \\
\hline AEO $87 \ldots \ldots \ldots \ldots$ & & & 1.83 & 1.95 & 2.11 & 2.28 & 4.07 & 0.78 \\
\hline AEO89 $\ldots \ldots \ldots \ldots \ldots$ & & & & 1.62 & 1.70 & 1.91 & 3.93 & 0.65 \\
\hline AEO90 $\ldots \ldots$ & & & & & 1.78 & 1.88 & 2.93 & 0.54 \\
\hline AEO91 $\ldots \ldots \ldots \ldots \ldots$ & & & & & & 1.77 & 2.51 & 0.49 \\
\hline AEO92 $\ldots \ldots \ldots \ldots \ldots$ & & & & & & & 2.35 & 0.76 \\
\hline AEO93 $\ldots \ldots \ldots \ldots \ldots$ & & & & & & & 2.29 & 0.70 \\
\hline Actual & 2.51 & 1.94 & 1.67 & 1.69 & 1.69 & 1.71 & 1.59 & \\
\hline \multicolumn{9}{|l|}{ Average Absolute } \\
\hline Error & 0.65 & 1.31 & 1.63 & 1.70 & 1.79 & 1.92 & 3.68 & 2.02 \\
\hline \multicolumn{9}{|c|}{ (percent error) } \\
\hline AEO82 & 72.4 & 182.1 & 300.0 & 344.2 & 376.1 & 401.5 & & 279.4 \\
\hline AEO83 $\ldots$ & 16.7 & 60.1 & 107.4 & 132.5 & 169.8 & 207.4 & 701.0 & 199.3 \\
\hline AEO84 $\ldots \ldots$ & 10.4 & 49.3 & 92.3 & 114.6 & 144.6 & 180.1 & 486.6 & 154.0 \\
\hline AEO85 & 3.6 & 34.4 & 59.0 & 60.2 & 74.3 & 95.9 & 319.4 & 92.4 \\
\hline AEO86 & & -10.8 & 17.2 & 35.4 & 50.5 & 64.4 & 270.8 & 74.8 \\
\hline AEO 87 & & & 9.6 & 15.2 & 24.6 & 33.5 & 156.3 & 47.8 \\
\hline AEO89 & & & & -4.1 & 0.6 & 11.4 & 147.2 & 40.9 \\
\hline AEO90 & & & & & 5.3 & 10.2 & 84.4 & 33.3 \\
\hline AEO91 & & & & & & 3.5 & 57.6 & 30.5 \\
\hline AEO92 $\ldots \ldots \ldots$ & & & & & & & 47.8 & 47.8 \\
\hline AEO93 $\ldots \ldots \ldots \ldots$ & & & & & & & 44.0 & 44.0 \\
\hline \multicolumn{9}{|l|}{ Average Absolute } \\
\hline Percent Error .... & 25.8 & 67.3 & 97.6 & 100.9 & 105.7 & 112.0 & 231.5 & 120.4 \\
\hline
\end{tabular}

AEO= Annual Energy Outlook.

Notes: AEO82 projections given in British thermal units; converted to trillion cubic feet using conversion factors provided in the Monthly Energy Review. Data for 1995 are preliminary.

Sources: History: Energy Information Administration (EIA), Monthly Energy Review, DOE/EIA-0035 (96/03) (Washington, DC, March 1996). Projections: EIA, various issues, Annual Energy Outlook, DOE/EIA-0383(82-93) (Washington, DC, April 1983 through January 1993).

were expected to overcome technological improvement in the more recent forecasts. In summary, three factors have had significant impacts on the projections:

- In the earlier AEOs, it was assumed that natural gas contracts whose provisions were governed by the Natural Gas Policy Act of 1978 would not be abrogated and that the prices that prevailed under those contracts would essentially set the market over time. In fact, when oil prices fell in 1986, many of those contracts were abrogated, and the price of natural gas fell, although not as much as the price of oil.

- Estimates of the recoverable resource base rose over time, in contrast to the assumptions in the forecast. Since the models use this information as an input, 
higher assumed levels of recoverable resources would have resulted in forecasts characterized by more gas available for production at lower prices.

- The earlier $A E O$ s did not assume that there would be additional competition in the transmission and distribution sectors of the market. However, from 1985 on, FERC moved to open access to the interstate pipeline transmission system, lowering end-use prices and stimulating additional price competition at the wellhead as well.

Thus, while the forecasts for 1995 improved with additional information, they continued to be affected by underestimates of the impacts of deregulation at the wellhead and the changing competitive structure of the industry and by overestimates of the impacts of depletion.

It is worth noting that much of the domestic production of natural gas is as a co-product of the crude oil extraction process, which means that, as crude oil production rises due to higher oil prices, there may be a depressing effect on the wellhead price of gas. This effect has added to the complexity of forecasting natural gas prices.

\section{Coal Prices to Electric Utilities}

While better than those of oil and gas, forecasts of coal prices to electric utilities still show an average error of 47 percent over the period studied (Table 15). All forecasts were overstated. The year with the highest average forecast errors was 1995, with projections averaging approximately double the actual price. There was, however, significant improvement in the 1995 forecast over time, with the error improving from 225 percent in the AEO 33 to 28 percent in the $A E O 93$. Across forecast years, the further out the forecast, the higher the average error, with the lowest error shown for the year 1985 at 15 percent.

The early AEOs-AEO82 through AEO85-tended to have the highest average error, exacerbated especially by their forecasts for 1995. There was steady improvement in the $A E O$ s through the $A E O 90$, which averaged a 21-percent error. After the AEO90, the overpredictions for 1995 made the overall average for subsequent AEOs more problematic.

The major factors in the overpredictions of coal prices were the assumptions concerning depletion effects, productivity improvements, capacity utilization, and transportation. Depletion was assumed to overcome productivity improvements in the long run; in fact, the onset of such new technology as longwall mines, together with the growth of surface mining in the West, has made depletion a much less important factor. Similarly, with high world oil price forecasts, the impacts of excess capacity and competition among existing mines were not seen to be as important as they in fact became. Finally, high world oil prices were assumed to affect both the production process and the costs of transportation. In fact, the collapse of oil prices in 1986 reduced the impact on both, and the increasing competitiveness of rail transportation has held transportation costs below expectations.

\section{Residential Electricity Prices}

Residential electricity prices, which are highly representative of electricity prices in general, showed the best forecasting record among the prices examined here, with an average error of 11 percent (Table 16). As with all of the price forecasts, the year with the highest average forecast errors was 1995, which had an average error of 25 percent. All of the other years' forecasts averaged 11 percent or lower. Except for 1985 and 1986, price forecasts have generally been higher than actual, with the exception of those made in the AEO86. By publication, the AEO83 had the highest average error, with the $A E O 86$ showing the lowest at less than 5 percent. The $A E O 86$ was also the only publication to underpredict the eventual price for all years except 1995 . The most recent $A E O$ s evaluated-AEO92 and $A E O 93$ - averaged under 10 percent for their 1995 forecasts.

The primary reason for high price forecasts was the impact of fuel costs and capital costs on expected prices. Fuel costs were consistently overestimated for oil, natural gas, and coal, and this had a strong effect on overestimating electricity prices, especially for the $A E O 82$ through the $A E O 84$. In addition, the costs of new capacity were assumed to be higher than actually occurred, and this assumption also helped to raise the forecasts. Finally, a 1992 study $^{20}$ on the accuracy of $A E O$ electricity forecasts for 1985 and 1990 indicated that prices were overestimated impart because of not incorporating public utility commission (PUC) disallowances and phase-ins of costs of some capital-intensive generating capacity. For example, some nuclear units had significant shares of their costs disallowed, while the remaining costs were phased in on a longer time schedule than the utilities had requested. Such PUC actions also contributed to lower-than-expected prices in some years.

${ }^{20 /}$ Forecasting Accuracy of the Electricity Market Model," prepared by Nuclear and Electricity Branch, Energy Information Administration, July 30, 1992, unpublished manuscript. 
Table 15. Coal Prices to Electric Utilities, Actual vs. Forecasts

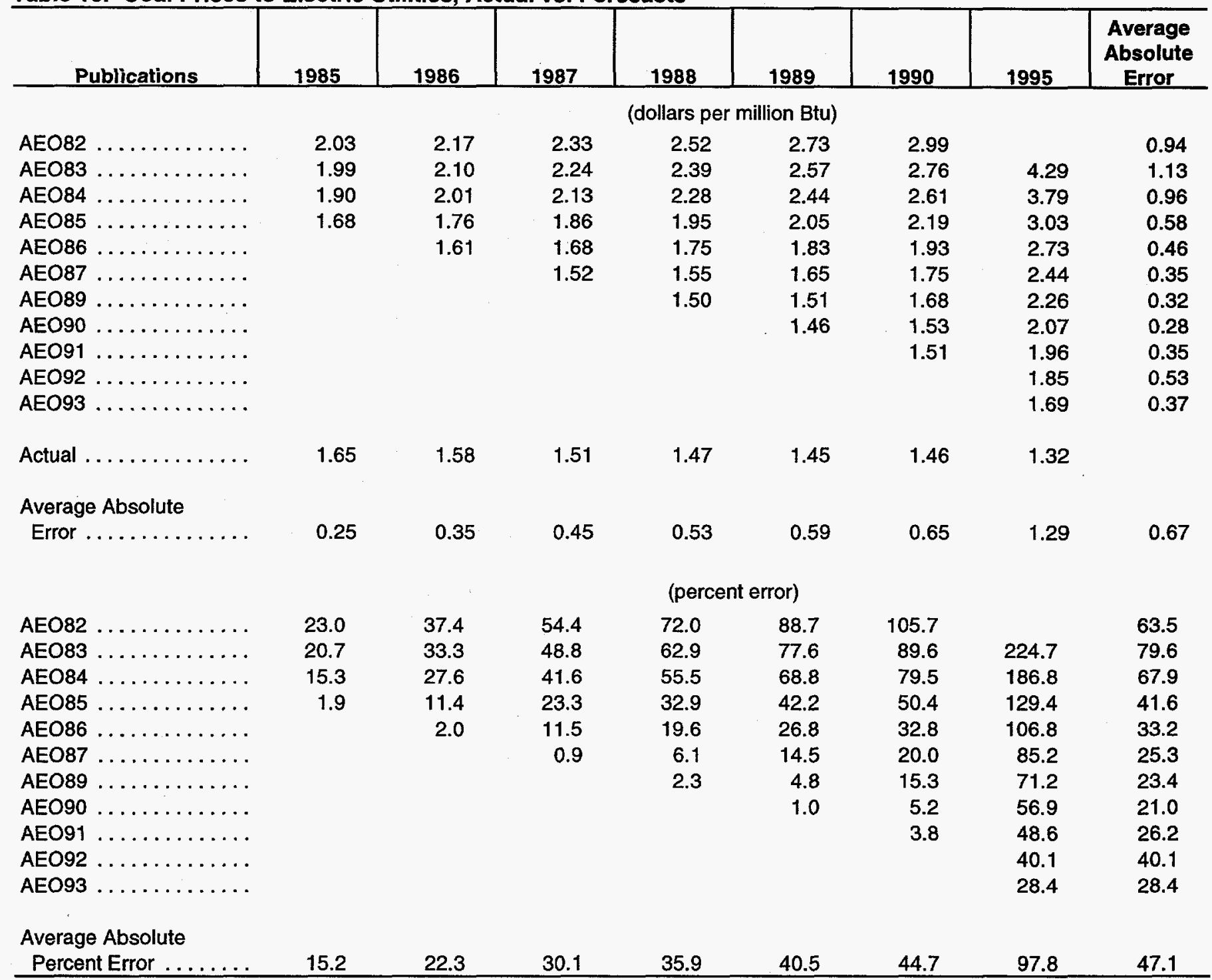

AEO = Annual Energy Outlook.

Btu $=$ British thermal unit.

Notes: Data for 1995 are preliminary.

Sources: History: Energy Information Administration (EIA), Monthly Energy Review, DOE/EIA-0035 (96/03) (Washington, DC, March 1996). Projections: EIA, various issues, Annual Energy Outlook, DOE/EIA-0383(82-93) (Washington, DC, April 1983 through January 1993).

\section{Disposable Personal Income}

The forecasts for disposable personal income (DPI) show an average error of 5 percent (Table 17). All of the forecasted years' projections have been less than 10 percent from actual, with the exceptions of the AEO83, AEO84, and AEO85 forecasts for 1995, which ranged from 17 to 27 percent above the actual DPI. For the years from 1985 through 1990, there are two distinct patterns: for AEO85 through $A E O 90$, the DPI forecasts tended to be understated for these years; for AEO82 through AEO84, the DPI forecasts were understated for the early years and overstated for the forecasts further out. For the year 1995, most of the forecasts were substantially overpredicted, except for the most recent ones-AEO91 through $A E O 93$ - which were either understated or, in the case of $A E O 93$, overstated by less than 1 percent of actual. 
Table 16. Residential Electricity Prices, Actual vs. Forecasts

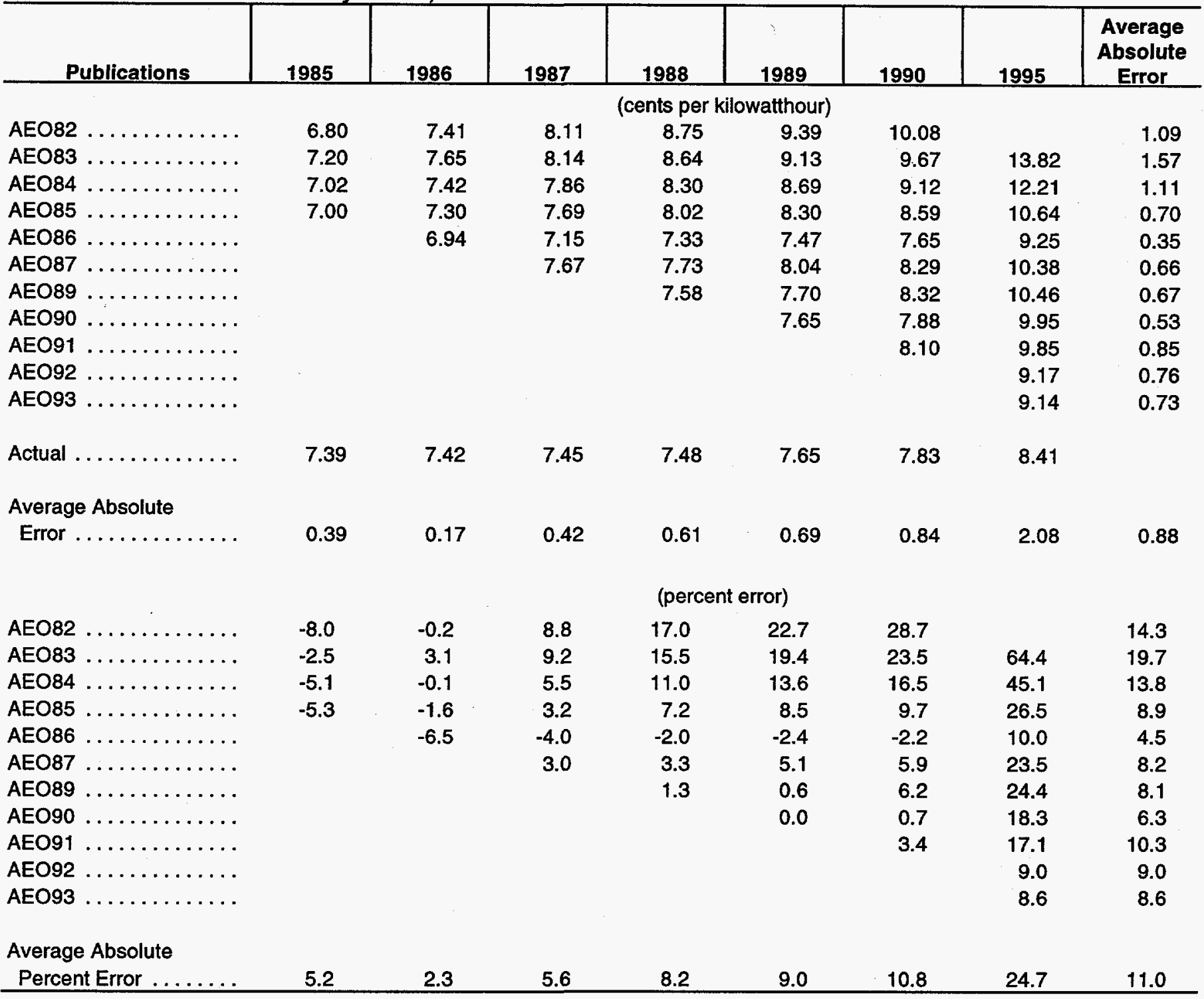

AEO = Annual Energy Outlook.

Notes: Data for 1995 are preliminary.

Sources: History: Energy Information Administration (EIA), Monthly Energy Review, DOE/EIA-0035 (96/03) (Washington, DC, March 1996). Projections: EIA, various issues, Annual Energy Outlook, DOE/EIA-0383(82-93) (Washington, DC, April 1983 through January 1993).

The major reason for the pattern of overprediction of the longer-term forecasts in the early $A E O$ s was the tendency to assume that the impacts of high world oil prices would eventually lessen and that reduced dependence on imported crude oil would help to stimulate the economy. While the impacts have lessened, the magnitude of the improvement has not been as high as expected in the early 1980 s. Conversely, the underestimates of the later $A E O$ s are due in part to the assumed impacts that deficit spending and higher interest rates would have on the national economy.
Interest rates have not risen as high as once feared, and the negative impact on the economy has not materialized.

\section{Conclusion}

This paper has presented an evaluation of the $A E O$ forecasts for the publications from 1982 through 1993. Clearly, the variables with the highest average forecast 
Table 17. Disposable Personal Income, Actual vs. Forecasts

\begin{tabular}{|c|c|c|c|c|c|c|c|c|}
\hline Publications & 1985 & 1986 & 1987 & 1988 & 1989 & 1990 & 1995 & $\begin{array}{c}\text { Average } \\
\text { Absolute } \\
\text { Error }\end{array}$ \\
\hline & \multicolumn{8}{|c|}{ (billion dollars) } \\
\hline AEO82 & 2,800 & 3,056 & 3,330 & 3,627 & 3,970 & 4,345 & & 164 \\
\hline AEO83 & 2,808 & 3,049 & 3,301 & 3,579 & 3,886 & 4,217 & 6,465 & 295 \\
\hline AEO84 & 2,786 & 2,978 & 3,224 & 3,499 & 3,807 & 4,132 & 6,231 & 257 \\
\hline AEO85 $\ldots \ldots \ldots \ldots \ldots$ & 2,776 & 2,934 & 3,156 & 3,409 & 3,697 & 4,002 & 5,976 & 236 \\
\hline AEO86 $\ldots \ldots \ldots \ldots$ & & 2,989 & 3,134 & 3,332 & 3,509 & 3,750 & 5,418 & 225 \\
\hline AEO87 $\ldots \ldots \ldots \ldots$ & & & 3,138 & 3,298 & 3,516 & 3,745 & 5,265 & 210 \\
\hline AEO $89 \ldots \ldots \ldots \ldots$ & & & & 3,383 & 3,601 & 3,864 & 5,523 & 217 \\
\hline AEO90 $\ldots \ldots \ldots \ldots$ & & & & & 3,671 & 3,865 & 5,316 & 147 \\
\hline AEO91 $\ldots \ldots \ldots \ldots$ & & & & & & 3,799 & 4,847 & 221 \\
\hline AEO92 & & & & & & & 4,815 & 280 \\
\hline AEO93 & & & & & & & 5,141 & 47 \\
\hline Actual & 2,985 & 3,161 & 3,290 & 3,533 & 3,763 & 3,993 & 5,095 & \\
\hline \multicolumn{9}{|l|}{ Average Absolute } \\
\hline Error .......... & 193 & 160 & 93 & 126 & 150 & 185 & 511 & 224 \\
\hline & \multicolumn{8}{|c|}{ (percent error) } \\
\hline AEO82 & -6.2 & -3.3 & 1.2 & 2.6 & 5.5 & 8.8 & & 4.6 \\
\hline AEOB3 & -5.9 & -3.6 & 0.3 & 1.3 & 3.3 & 5.6 & 26.9 & 6.7 \\
\hline AEO84 $\ldots \ldots \ldots$ & -6.7 & -5.8 & -2.0 & -1.0 & 1.2 & 3.5 & 22.3 & 6.1 \\
\hline AEO85 $\ldots \ldots \ldots \ldots$ & -7.0 & -7.2 & -4.1 & -3.5 & -1.7 & 0.2 & 17.3 & 5.9 \\
\hline AEO86 $\ldots \ldots \ldots \ldots$ & & -5.4 & -4.7 & -5.7 & -6.8 & -6.1 & 6.3 & 5.8 \\
\hline AEO $87 \ldots \ldots \ldots \ldots$ & & & -4.6 & -6.6 & -6.6 & -6.2 & 3.3 & 5.5 \\
\hline AEO89 & & & & -4.2 & -4.3 & -3.2 & 8.4 & 5.0 \\
\hline AEO90 $\ldots \ldots \ldots \ldots$ & & & & & -2.4 & -3.2 & 4.4 & 3.3 \\
\hline AEO91 $\ldots \ldots \ldots \ldots$ & & & & & & -4.9 & -4.9 & 4.9 \\
\hline AEO92 & & & & & & & -5.5 & 5.5 \\
\hline$\ldots \ldots \ldots \ldots$ & & & & & & & 0.9 & 0.9 \\
\hline \multicolumn{9}{|l|}{ Average Absolute } \\
\hline Percent Error & 6.5 & 5.1 & 2.8 & 3.6 & 4.0 & 4.6 & 10.0 & 5.4 \\
\hline
\end{tabular}

$\mathrm{AEO}=$ Annual Energy Outlook.

Notes: Data for 1995 are preliminary.

Sources: History: Energy Information Administration (EIA), Monthly Energy Review, DOE/EIA-0035 (96/03) (Washington, DC, March 1996). Projections: ElA, various issues, Annual Energy Outlook, DOE/EIA-0383(82-93) (Washington, DC, April 1983 through January 1993).

errors have been natural gas prices at the wellhead, world oil prices, and net petroleum and natural gas imports. Natural gas, in general, has been the fuel with the most inaccurate forecasts, showing the highest average error for consumption, production, imports, and prices. In some ways, this is not surprising. Natural gas was the last fossil fuel to be deregulated following the heavy regulation of energy markets in the 1970s and early 1980s and was assumed in the early $A E O$ s to continue to be regulated until new rules were actually promulgated. Even after deregulation, the behavior of natural gas was difficult to predict in competitive markets.

The overprediction of prices is the most striking feature of this evaluation. In general, technological improvements that were higher than expected, the demise of OPEC's market power, excess productive capacity, and market competitiveness were the factors that the $A E O$ forecasts 
failed to anticipate. While the errors for prices were large, they appeared to have a relatively minor impact on overall predictions for demand and production, although there were some forecasts that were clearly affected. One possible conclusion is that relatively low price elasticities of supply and demand embedded in the models may be confirmed by this result. However, another possible conclusion is that the higher price projections helped to offset elasticities that were too low and that the models' elasticities may not have reflected the adjustment in demands that were made in the face of the higher energy prices that resulted after two oil disruptions. In either case, it is clear that, at least for the period covered by this study, productivity and technology improvements, as well as the effects of gradual deregulation and changes in the structure of the industry, such as the treatment of contracts, have more than offset the factors that tend to raise fossil fuel prices, especially for natural gas and coal. 



\title{
Differences Between Energy Information Administration Energy Forecasts: Reasons and Resolution
}

\author{
by Arthur T. Andersen
}

\section{Introduction}

This paper describes recent efforts to improve the comparability and performance of the Energy Information Administration's (EIA) two forecasting systems that project integrated U.S. energy market activities. ELA provides periodic projections of the production, consumption, pricing, and distribution of energy products in the United States and associated international energy markets. Shortterm forecasts, with quarterly projections, are provided for up to eight quarters into the future and are published in the Short-Term Energy Outlook (STEO). ${ }^{1}$ The Annual Energy Outlook $(A E O),{ }^{2}$ published every January, contains annual projections for up to twenty years into the future.

The short-term and midterm forecasts are based on different modeling systems, which have different objectives. The short-term projections are designed to capture the most recent trends in energy market activity and the effects on these trends of important near-term transient market influences such as weather changes and variations in economic activity. The midterm forecasts are concerned with factors such as changes in technology and structural changes in energy markets or the economy that will influence energy markets over a longer time period.

The two systems each give projections for the current year and for the next. The forecasts for these overlapping years were compared in order to investigate the differences between their projections. Based on the findings from the comparisons, the modeling systems were revised with the goal of reducing the differences between the projections of the two systems and improving the performance of both.

The STEO forecasts are EIA's official energy market forecasts for the remainder of the current year and for the next year because the short-term forecasting system has been explicitly designed to provide forecasts that are responsive to changes in current conditions which have immediate impacts on energy markets. The midterm forecasting system is based on average long-term weather conditions and economic activity and thus is not expected to provide short-term forecasts that are identical to those in the STEO.

Thus, the forecasts in the STEO and AEO are not the same for the time periods over which they overlap without providing a method for calibrating the results to make them agree. Even when the STEO projections are normalized to average weather conditions and to economic assumptions consistent with those in the $A E O$, there still remain some differences, in a few cases substantial, between the two forecasts. In 1995, EIA undertook a project involving the staff responsible for the STEO and AEO forecasts as well as other industry experts within EIA to explore the sources of the differences and to recommend changes where appropriate in each modeling system to minimize the differences in their views of energy markets in the near term. This paper discusses the results of this project, including a discussion of the differences found, the reasons for them, and the changes made to the modeling systems to bring the short-term projections from each system into closer alignment. The paper focuses upon the efforts to align the projections of energy consumption and production.

'Energy Information Administration, Short-Term Energy Outlook 1996 2nd Quarter, DOE/EIA-0202(96/2Q) (Washington, DC, April 1996) for the most recent STEO.

${ }^{2}$ Energy Information Administration, Annual Energy Outlook 1996, DOE/EIA-0383(96) (Washington, DC, January 1996) for the most recent AEO. 


\section{Overview of the Two Modeling Systems}

The short-term forecasts presented in the STEO are prepared using the Short-Term Integrated Forecasting System (STIFS) ${ }^{3}$ while the midterm forecasts in the $A E O$ are based upon the National Energy Modeling. System (NEMS). ${ }^{4}$ The two modeling systems are based on quite different methodologies reflecting the different purposes each system is designed to serve.

STIFS projects energy market statistics at the national level for quarterly or monthly time periods. The central purpose of STIFS is to capture recent trends in energy market activity and the influence of changes in those factors that can have a substantial short-run effect on these markets. Consequently, STIFS contains a series of equations for fairly aggregate energy concepts-total electricity consumption for the Nation in the residential sector, for example. These equations incorporate time trends and the effects on these trends of weather, economic activity, and other short-term influences.

NEMS provides regional projections on an annual basis. The midterm forecasts are intended to evaluate the effects of factors that influence energy markets over a number of years, including the influences of changing technologies throughout the energy system, the role of regulations and policies on various segments of energy markets, ${ }^{5}$ and the effect of structural changes in the U.S. economy. To meet these requirements, NEMS contains a much more detailed description of energy market activities than is required for STIFS. For example, within NEMS, total electricity consumption for the Nation in the residential sector is obtained by summing residential electricity consumption in each of the nine regions represented in NEMS. Within a given region, residential electricity consumption is determined by summing electricity usage over all the major applications for which electricity is used-space heating, air-conditioning, water heating, lighting, cooking, and other appliances. For each end-use application, consumption is estimated for several categories of capital equipment, with the stock of equipment updated for each year of the forecast.
Each area of energy supply, distribution, and consumption in NEMS is represented by a detailed methodology similar to that described for residential electricity demand.

\section{Comparisons of the Two Modeling Systems}

The comparison of the forecasts from the two systems started with an examination of how the elements of energy markets are defined in each system to look for inconsistencies. For example, each system has projections of total world oil production. However, NEMS had not included production in the formerly Centrally Planned Economies (CPE)-the former Soviet Union, Eastern Europe, and China-in this total, but this production is included in STIFS. EIA's midterm forecasts had been projecting net oil exports for the CPEs rather than making separate estimates of consumption, production, imports, and exports. This was done because of data limitations.

Another source of difference is the aggregation procedures used in the data series used for parameter estimations. Price information is collected from all or a sample of vendors, and these data are aggregated to determine an average price for a region or the Nation. Different methods can be used for the aggregation, which could lead to differences in the data series. Since STIFS and NEMS provide projections at different levels of detail, aggregation typically must be performed in order to compare the two projections. This aggregation is another potential source of differences in the two forecasts. In order to compare STIFS and NEMS projections, STIFS solutions must be aggregated from a quarterly to an annual basis, and NEMS solutions must be aggregated from regional to national totals. Fuel prices reported by NEMS at the national level are quantity-weighted averages of regional fuel prices-the average national price is calculated by summing the product of the price and the quantity consumed for each region and then dividing by the total consumption. Because the national average price distribution of consumption in NEMS depends upon levels of regional consumption as well as regional prices, a difference between STIFS and NEMS in a price could be a result of the regional distribution of consumption in NEMS, as well as the regional prices in NEMS.

${ }^{3}$ Energy Information Administration, Model Documentation Report: Short-Term Integrated Forecasting System, DOE/EIA-M030 (Washington, DC, May 1993).

${ }^{4}$ Energy Information Administration, The National Energy Modeling System: An Overview, DOE/EIA-0581(96) (Washington, DC, March 1996), plus model documentation reports on the individual modules of NEMS available from the National Energy Information Center.

'The baseline projections in the $A E O$ are made under the assumption that energy policies and regulations currently in place will remain in effect throughout the forecast period. However, the effective date of some of these regulations may be a future year, such as a requirement that the energy efficiency of a specific appliance be increased by a specified amount in a future year. These regulations are incorporated in NEMS. In addition, NEMS is used to evaluate the effects of proposed alternative policies and other assumptions as requested. 
In addition to differences caused by definitional differences or aggregation, there can exist differences in modeling approaches, common input assumptions, and the historical data sources used for estimating forecast equation parameters. An evaluation of all these areas was conducted in comparing the 1994 and 1995 projections in the Annual Energy Outlook 1995 (AEO95) ${ }^{6}$ with the comparable STEO projections.

\section{Comparisons of 1994 and 1995 Forecasts}

Prior to this project, differences between the STEO and $A E O$ forecasts for the years in which they overlapped were typically suppressed in the published $A E O$ forecasts. Either the midterm forecasting system produced a forecast that agreed with the STEO forecast or the results were overwritten to agree with the STEO results-this forced agreement is termed benchmarking. Benchmarking was included in the published results for 1994 in AEO95. After NEMS was completed, this project was started to explore and reduce to the extent possible the differences between the two forecasts in order to improve them both. The reasons for differences found ranged from straightforward differences in the definitions used for data series to more subtle reasons having to do with how both models capture changes in the trends underlying energy product production and consumption.

For the evaluation of differences between the two forecasts, two sets of comparisons were made. First, in early 1995, the NEMS reference case results for 1994 and 1995 published in AEO95, with benchmarking eliminated for $1994,{ }^{7}$ were compared with corresponding STEO results published in the Short-Term Energy Outlook 1994 3rd Quarter (STEO3Q(94)). ${ }^{8}$ Based upon differences found in this comparison, a detailed evaluation of the reasons for the differences was made, and changes to one or both modeling systems were made as appropriate in an effort to reduce the differences. Changes were made to the NEMS system being prepared to conduct the forecasts for Annual Energy Outlook 1996 (AEO96) $^{9}$ and to the STIFS to be used for the Short-Term Energy Outlook 1995 3rd Quarter (STEO3Q(95))..$^{10}$ The second comparison was between the $A E O 96$ results and STEO3Q(95) results which incorporated these changes.
Table 1 presents a summary of the differences between NEMS and STIFS results for these two comparisons. The results indicate that the efforts undertaken to reduce discrepancies were successful; however, differences still remain, and the process of comparing model solutions will continue. The detection and explanation of differences help the understanding of the strengths and weaknesses of each modeling approach and have become an important resource in the model evaluation and improvement cycles of both NEMS and STIFS. The goal was to bring the differences to 2 percent or less for the major series shown in Table 1. In those cases where the difference was on that order, no additional effort was made to bring the forecasts into closer agreement even if the differences were greater for $A E O 96$ than they were for $A E O 95$ (see transportation consumption, for example).

Comparisons were made for 53 commonly reported series. In the comparison of AEO95 and STEO3Q(94) for the first year of the forecast, differences were less than 5 percent for 31 of the series, in the range of 5 to 10 percent for 8 series, and in excess of 10 percent for 14 series (Table 1). In the second year of the forecast, 27 series had less than a 5percent difference and 12 series were in the 5- to 10percent range. In the second comparison, made after the model adjustments were completed to correct for the discrepancies found in the modeling systems, 46 of the series had a difference less than 5 percent for the first year of the forecast and 44 series were less than 5 percent for the second year.

\section{Factors Contributing to Discrepancies Between NEMS and STIFS}

The energy series compared in the STEO and $A E O$ forecasts include total production of individual fuels, total consumption by individual fuel, total consumption by economic sector, and consumption of individual fuels within each economic sector. Table 2 presents some of the differences between AEO95 and STEO3Q(94) for projections for 1994 and 1995.

${ }^{6}$ Energy Information Administration, Annual Energy Outlook 1995, DOE/EIA-0383(95) (Washington, DC, January 1995).

The NEMS run used for the comparison was prepared in February 1995 ex post to represent NEMS results for $A E O 95$ without any of the adjustments for STEO3Q(94) projections. This solution is referred to as the unbenchmarked NEMS solution.

${ }^{8}$ Energy Information Administration, Short-Term Energy Outlook 1994 3rd Quarter, DOE/EIA-0202(94/3Q) (Washington, DC, August 1994).

${ }^{9}$ Energy Information Administration, Annual Energy Outlook 1996, DOE/EIA-0383(96) (Washington, DC, January 1996).

${ }^{10}$ Energy Information Administration, Short-Term Energy Outlook 1995 3rd Quarter, DOE/EIA-0202(95/3Q)(Washington, DC, August 1995). 
Table 1. Comparisons of NEMS and STIFS Projections for Major Series ([NEMS - STIFS] as a percent of STIFS)

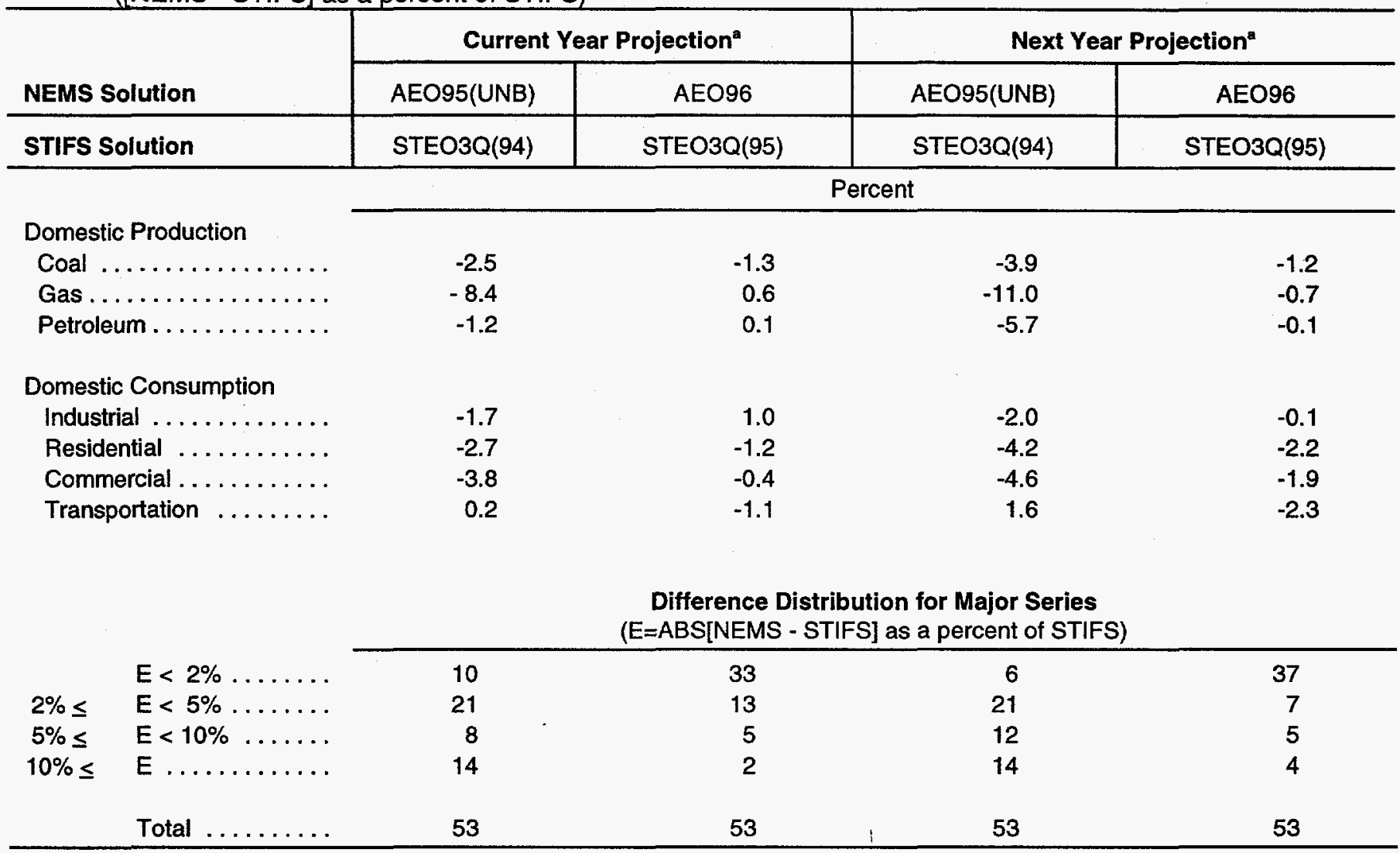

${ }^{2}$ For AEO95 and STEO3Q(94) the Current Year is 1994 and the Next Year is 1995; for AEO96 and STEO3Q(95) the Current Year is 1995 and the Next Year is 1996.

AEO = Annual Energy Outlook.

NEMS = National Energy Modeling System.

STEO = Short-Term Energy Outlook.

STIFS $=$ Short-Term Integrated Forecasting System

Notes: The AEO95 projections are the unbenchmarked NEMS solution described above. The differences provided are [NEMS STIFS] as a percent of STIFS.

Sources: Energy Information Administration (EIA), Annual Energy Outlook 1995, DOE/EIA-0383(95) (Washington, DC, January 1995); EIA, Annual Energy Outlook 1996, DOE/EIA-0383(96) (Washington, DC, January 1996); EIA, Short-Term Energy Outlook 1994 3rd Quarter, DOE/EIA-0202(94/3Q) (Washington, DC, August 1994); EIA, Short-Term Energy Outlook 1995 3rd Quarter, DOE/EIA0202(95/3Q) (Washington, DC, August 1995).

While the aggregate energy consumption totals were quite close, less aggregated consumption estimates showed greater variation. Moreover, some of the differences were markedly larger in the second year of the forecasts. Many of the differences could be related to one of three basic reasons.

Differences in Series Definitions. For some series, the same terminology was used to describe the series, but there were differences in the definitions of the energy series reported in the two forecasting systems. The principal examples are:
World Petroleum Production: As reported by STIFS, this series includes production in China, the former Soviet Union, and Eastern Europe, while the series reported by NEMS did not since NEMS forecasts net exports for these countries.

AMTRAK Consumption of Electricity: This consumption in NEMS is included as part of transportation sector electricity consumption, while in STIFS it is part of commercial sector consumption, consistent with the way consumption data are reported to EIA by electric utilities. 
Table 2. NEMS and STIFS Projections in 1994 and Percent Differences (Quadrillion Btu)

\begin{tabular}{|c|c|c|c|c|c|c|}
\hline \multirow[b]{2}{*}{ Energy Indicators } & \multicolumn{3}{|c|}{ For 1994 Prepared in 1994} & \multicolumn{3}{|c|}{ For 1995 Prepared in 1994} \\
\hline & STEO3Q(94) & AEO95 & Percent & STEO3Q(94) & AEO95 & Percent \\
\hline \multicolumn{7}{|l|}{ Production } \\
\hline Coal .... & 22.17 & 21.44 & -3.31 & 22.30 & 21.65 & -2.91 \\
\hline Gas....... & 19.33 & 18.83 & -2.57 & 19.97 & 18.52 & -7.24 \\
\hline Petroleum ............. & 17.34 & 17.13 & -1.23 & 17.40 & 16.53 & -5.01 \\
\hline Net Imports & 18.51 & 18.94 & 2.30 & 18.65 & 19.90 & 6.72 \\
\hline Total Supply ......... & 86.94 & 89.74 & 3.23 & 88.04 & 90.38 & 2.66 \\
\hline Losses & -21.61 & -22.28 & 3.10 & -21.86 & -22.37 & 2.35 \\
\hline Net Supply & 65.33 & 67.46 & 3.27 & 66.18 & 68.01 & 2.76 \\
\hline \multicolumn{7}{|l|}{ Consumption } \\
\hline Industrial & 26.80 & 26.41 & -1.44 & 27.19 & 26.55 & -2.36 \\
\hline Residential & 10.95 & 10.96 & 0.09 & 10.98 & 10.79 & -1.75 \\
\hline Commercial . & 7.10 & 6.93 & -2.38 & 7.22 & 6.91 & -4.32 \\
\hline Transportation $\ldots \ldots \ldots$ & 23.27 & 23.26 & -0.03 & 23.69 & 24.02 & 1.39 \\
\hline Total Consumption ... & 68.11 & 67.56 & -0.81 & 69.08 & 68.27 & -1.18 \\
\hline Discrepancy ............ & 2.79 & 0.10 & & 2.90 & 0.26 & \\
\hline
\end{tabular}

AEO = Annual Energy Outlook.

Btu $=$ British thermal unit.

NEMS = National Energy Modeling System

STEO = Short-Term Energy Modeling System .

STIFS $=$ Short-Term Integrated Forecasting System .

Note: The differences are [NEMS - STIFS] as a percent of STIFS.

Sources: Energy Information Administration (EIA), Short-Term Energy Outlook 1994 3rd Quarter, DOE/EIA-0202(94/3Q) (Washington, DC, August 1994); EIA, Annual Energy Outlook 1995, DOE/EIA-0383(95) (Washington, DC, January 1995).

Natural Gas Production: Data collected for total natural gas supply are historically greater than data collected for total natural gas disposition, resulting in a consistently positive accounting discrepancy ${ }^{11}$ over the recent historical time period. STIFS forecasts a positive discrepancy, while NEMS had assumed a discrepancy of zero for $A E O 95$. A positive natural gas discrepancy was incorporated for all forecast years within NEMS for AEO96, contributing to the decrease in the difference in the natural gas production forecast between NEMS and STIFS.

Gasoline Prices: Differences in the data systems utilized for the different systems lead to a difference in the gasoline prices for the two systems, with the data used for STIFS typically about $\$ .05$ per gallon higher than the data system supporting NEMS. The STIFS projections are based on a Bureau of Labor Statistics (BLS) series, while NEMS uses the retail gasoline series from EIA's Petroleum Marketing Annual (PMA). ${ }^{12}$ The BLS series is not volumeweighted and is based on a sample of urban retail outlets, while the PMA series is volume-weighted using statelevel data. The BLS series also includes a small amount of local taxes not reflected in the $P M A$ series.

Some of the differences in series definitions led to fairly large forecast differences. Many of them could be dealt with immediately or, as in the case of AMTRAK, explained. However, as discussed below, some of the definitional problems are subtle and relate to the impact of changes in energy markets upon the compositional detail reported to EIA in the monthly and annual energy data survey reports.

\footnotetext{
${ }^{11} \mathrm{Natural}$ gas lost as a result of converting flow data measured at varying temperatures and pressures to a standard temperature and pressure and the merger of different data reporting systems.

${ }^{12}$ Energy Information Administration, Petroleum Marketing Annual, DOE/EIA-0487.
} 


\section{Differences in Economic Sector Definitions.}

The historical data series used as the bases for the projections of energy consumption by economic sector in STIFS are the fuel-specific and economic sector-specific data published by EIA. These data have been obtained for the most part from energy suppliers or distributors. As is customary for short-term forecasting, the STIFS projections are essentially extrapolations of these historical data series with adjustments for those factors known to impact energy markets in the near term. As a result, the economic sectors in the STIFS projections correspond to the definitions of these sectors implicit in the EIA energy data series.

In collecting the energy data from suppliers, EIA provides a definition of each sector that is consistent with the standard economic definition of the sector. However, EIA also allows the utilities and other data suppliers to use their own definition of a sector if their data are organized by a different definition and if it is more feasible for them to provide the data in accordance with that definition. Many utilities have separate rate schedules for each sector-residential, commercial, and industrial. Customers are often assigned to a rate schedule on the basis of their consumption level rather than their economic activity. Consumption within one sectoral category may be billed, and hence measured, in another category. For example, a large residential electricity user (e.g., a large apartment building) might be billed at a commercial rate and that consumption would therefore be measured in the EIA data system and reflected in STIFS as commercial consumption, while apartment building consumption is included in the residential sector in NEMS. Furthermore there is variation across utilities in their rate structures, so the classification of consumption by sector varies from utility to utility.

Another factor that confuses sector identification is the recent increase in nonutility power generation, particularly cogeneration by industrial users. All of the fuel that a utility supplies to an industrial firm will be listed as industrial consumption when that utility responds to a fuel supply survey. It is not possible for the utility to know for what purposes the fuel is used. If an industrial user burns some of the fuel for cogeneration, this use cannot be distinguished from the fuel used for direct industrial applications. This limitation of the accounting system does not provide any information for allocating the user's consumption between cogeneration and other industrial uses.

Because of the purposes of NEMS and the data sources upon which it is based, the economic sector definitions in NEMS are much closer to the standard economic definitions than is possible for STIFS. A major purpose of NEMS is to analyze the energy system impacts of energy policies and changes in energy-using technologies, and the projections are designed to show the relationship between energy consumption and the efficiencies and inventories of energy-consuming devices specific to each economic sector. NEMS projects annual changes in the inventories and characteristics of equipment and their intensity of use, providing a link between economic and technological changes and energy consumption patterns.

Technology detail for energy consumption is provided for the residential, commercial, industrial, and transportation sectors. The baseline information in the consumption modules in NEMS is heavily dependent on data from EIA's energy consumption surveys and other data sources that provide sector-specific information about the stock of energy-using equipment and its characteristics. EIA's energy consumption surveys are conducted every three years, which limits the frequency with which some of the technology information in the NEMS consumption modules can be updated. The supply surveys used for STIFS are conducted at least annually, and many are monthly, which allows a more frequent update of the STIFS forecast parameters.

Because of the differences in the data sources underlying STIFS and NEMS, their definitions of the end-use energy consuming sectors differ. There is no alternative, independent source of measures of energy consumption for each sector, so it is not possible to make a judgement about how far the estimates for each system might deviate from the true consumption for the sector. This situation is likely to become exacerbated in the future as deregulation of electric utilities and natural gas distributors allows power generators and natural gas pipeline companies to deal directly with customers, bypassing the current utilities. Currently, a variety of adjustments are made to the projections so that total consumption figures agree with EIA's published totals on energy consumption. Total electricity consumption in the residential sector, for example, as initially determined by NEMS for a historical year, will be scaled up or down as necessary so that the total from NEMS agrees with EIA's published total based upon the surveys of electric utilities.

Differences in Modeling Approaches. Each model is organized to capture market changes in different ways. STIFS captures changes that are implicitly reflected in the time iiends in energy consumption, production, and the other values that STIFS projects. Returning to the earlier example of residential electricity consumption, there are many reasons why electricity consumption changes over time, including changes in the size of housing as new homes are built and older ones are remodeled or removed from the housing stock; purchases of newer, more efficient appliances to replace old ones; additions of new appliances to the total appliance stock; 
and increased market penetration of air conditioning. All such changes are reflected in the time series on residential electricity consumption that are the basis for the STIFS projections.

In contrast, NEMS represents all the major uses for energy in the residential sector and has procedures for representing the changes in housing characteristics and appliance stocks that lead to changes in electricity consumption. Time trends in electricity consumption in NEMS are derived from changes in these factors.

The extrapolation of time series information used in STIFS is particularly appropriate for short-term models as changes in the characteristics of the energy-consuming equipment usually occur slowly over time, so a substantial portion of these changes is readily captured in the time trends. In the longer term, though, significant shifts in a sector-such as substantial penetration of a highly energyefficient appliance, the introduction of new energy-using equipment, or the results of compliance with new regulations on efficiency-can cause energy consumption to shift from the historical time trends. For these circumstances, the technology detail in NEMS becomes a more effective vehicle for capturing these changes and is the modeling approach of choice for longer-term modeling.

In attempting to align NEMS and STIFS for short-term projections, two important considerations come into play. First, STIFS is implicitly picking up current trends in important determinants of energy consumption, such as in the average size of new houses. In contrast, NEMS will typically update information on stocks of equipment and its characteristics from surveys of energy use or housing. These survey results are generally available only after some delay as the surveys take time to complete. Consequently, some important data sources for NEMS will lag those for STIFS. Second, NEMS must have a full accounting of all the ways in which energy is used and some adjustments must be made for differences in sectoral definitions to provide a meaningful comparison with STIFS.

The growth in residential electricity consumption for the two years 1994 and 1995 was higher in STIFS than in NEMS. To assess the possible reasons for the difference, several steps had to be taken. First, the time trend projected by STIFS was evaluated to see whether there were some unusual features in the recent historical data that might have lead to an overestimation in the STIFS projections. Within NEMS, all the components of electricity consumption within the residential sector and across the different regions had to be evaluated to determine whether there might be trends in some of the energy-consuming stock or its characteristics that were not adequately represented within NEMS. Needless to say, this process required a detailed evaluation of a wide range of model parameters. The resolution of the differences is discussed below.

A similar process of evaluating the basis for the projections in the two systems was carried out for each of the consumption and production sectors. In a few cases, the process of comparison can be more simply described. The projected volume of Canadian natural gas imports serves as an example. NEMS represents these imports by starting with a pipeline capacity forecast and assuming a maximum annual utilization. Due to the relatively low costs of natural gas produced in Canada, NEMS typically solves for import values corresponding to these maximum utilization rates. To align the NEMS forecasts with STIFS, these rates were adjusted. Further, STIFS projections of import levels were adjusted upward to reflect planned capacity expansions.

\section{Changes Undertaken for the AEO96 and Their Impacts}

A substantial effort was devoted to better coordinate the NEMS and STIFS projections and reduce differences for the $A E O 96$ production cycle. A variety of changes were undertaken to reduce or eliminate differences resulting from the various reasons itemized above. A summary of these is given below.

For energy demand:

- NEMS residential sector demand was increased to account for a trend of more square feet in new houses. STIFS residential sector electricity demand was decreased to correspond to the NEMS projection of increased efficiency in electricity consumption.

- NEMS residential electricity demand was increased to reflect recent growth in electricity-based consumer products such as personal computers.

- NEMS projections of refinery natural gas consumption, measured as part of the industrial sector energy consumption, were increased through modeling changes and the respecification of the definitions of some fuel series.

- Product detail in petroleum demand was added to STIFS to assist in comparisons with NEMS results on a sectoral and fuel-specific basis. 
For energy supply:

- A representation of coal blending at electric utilities was incorporated into NEMS, removing the previous constraint on burning subbituminous coal at most electricity generating plants. As a result, there was a much closer agreement between STIFS and NEMS on the total quantity of coal produced.

- NEMS added a projection of the historical discrepancy for natural gas to projected natural gas consumption consistent with a similar procedure used for STIFS and included in the data series upon which STIFS is based. Since this discrepancy was also added to production, there was much closer agreement between STIFS and NEMS on gas production.

- The methodology for determining the volume of natural gas imports from Canada was coordinated between NEMS and STIFS to insure that both systems give similar forecasts.

- NEMS projections of electricity transmission and distribution $(T \& D)$ losses were increased, based on a calibration of regional North American Electricity Reliability Council data to the aggregate national data published by EIA. As a result, short-term T\&D losses were increased, which led to higher levels of production for gas and coal to meet the increased generation requirements.

- NEMS added projections of gas consumption used for flame stabilization in coal-fired electricity generation plants, consistent with STIFS.

For international oil markets:

- NEMS was revised to include projections of oil production in the former Soviet Union, Eastern Europe, and China to correspond with the STIFS procedures.

The process for comparing NEMS and STIFS solutions for the series published in STEO was automated. As the NEMS reference case was developed, differences between NEMS and STIFS solutions could be readily monitored.

The results of these changes and the corresponding reduction in forecast differences are summarized in Table 1. Table 3 gives a comparison between forecasts for years 1995 and 1996 prepared in 1995 for AEO96 and for both third and fourth quarter STEO projections. Comparison of the differences indicates that there are no substantial differences between the comparisons between the $A E O$ projections and either quarter of the STEO projections. While some differences remain, differences are expected and many are inconsequential. 
Table 3. NEMS and STIFS Projections in 1995 and Percent Differences (Quadrillion Btu)

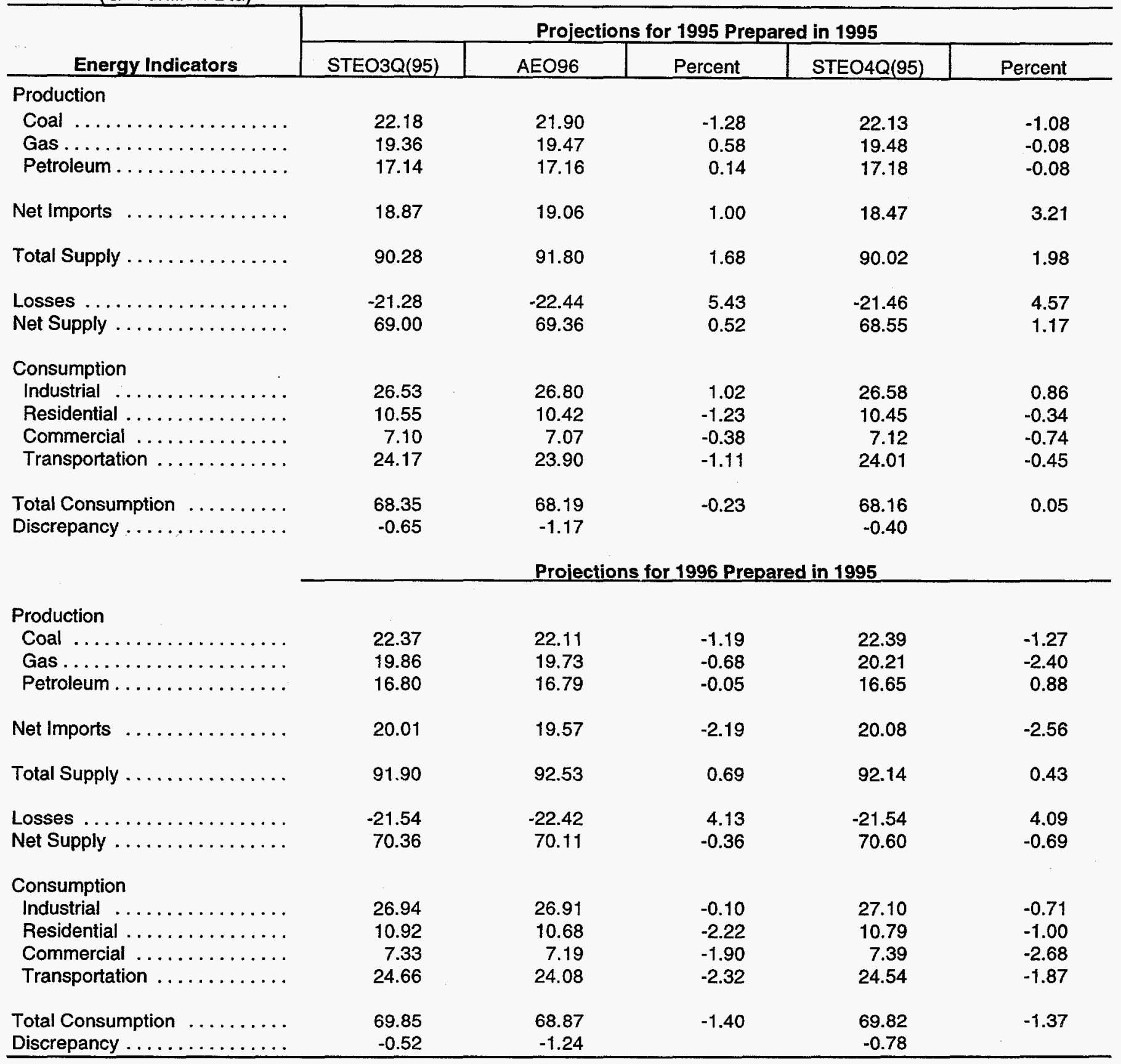

AEO = Annual Energy Outlook.

Btu $=$ British thermal unit.

NEMS = National Energy Modeling System.

STEO = Short-Term Energy Outlook.

STIFS = Short-Term Integrated Forecasting System.

Note: The differences are [NEMS - STIFS] as a percent of STIFS.

Sources: Energy Information Administration (EIA), Short-Term Energy Outlook 1995 3rd Quarter, DOE/EIA-0202(95/3Q) (Washington, DC, August 1995); EIA, Annual Energy Outlook 1996, DOE/EIA-0383(96) (Washington, DC, January 1996); EIA, Short-Term Energy Outlook 1995 4th Quarter, DOE/EIA-0202(95/4Q) (Washington, DC, November 1995). 
